\title{
The Number of Irreducible Polynomials Over a Finite Field With Prescribed Coefficients
}

by

Behzad Omidi Koma

M.Sc., Sharif University, 1995

A thesis submitted to the Faculty of Graduate and Postdoctoral Affairs in partial fulfillment of requirements of the degree of

\section{Doctor of Philosophy}

in

Applied Mathematics

Carleton University

Ottawa, Ontario

(c) 2010, Behzad Omidi Koma 


$\begin{array}{ll}\begin{array}{l}\text { Library and Archives } \\ \text { Canada }\end{array} & \begin{array}{l}\text { Bibliothèque et } \\ \text { Archives Canada }\end{array} \\ \begin{array}{l}\text { Published Heritage } \\ \text { Branch }\end{array} & \begin{array}{l}\text { Direction du } \\ \text { Patrimoine de l'édition }\end{array} \\ \begin{array}{l}\text { 395 Wellington Street } \\ \text { Ottawa ON K1A ON4 }\end{array} & \begin{array}{l}395, \text { rue Wellington } \\ \text { Ottawa ON K1A ON4 } \\ \text { Canada }\end{array} \\ \text { Canada }\end{array}$

Your file Votre référence ISBN: 978-0-494-70557-5 Our file Notre référence ISBN: 978-0-494-70557-5

NOTICE:

The author has granted a nonexclusive license allowing Library and Archives Canada to reproduce, publish, archive, preserve, conserve, communicate to the public by telecommunication or on the Internet, loan, distribute and sell theses worldwide, for commercial or noncommercial purposes, in microform, paper, electronic and/or any other formats.

The author retains copyright ownership and moral rights in this thesis. Neither the thesis nor substantial extracts from it may be printed or otherwise reproduced without the author's permission.
AVIS:

L'auteur a accordé une licence non exclusive permettant à la Bibliothèque et Archives Canada de reproduire, publier, archiver, sauvegarder, conserver, transmettre au public par télécommunication ou par l'Internet, prêter, distribuer et vendre des thèses partout dans le monde, à des fins commerciales ou autres, sur support microforme, papier, électronique et/ou autres formats.

L'auteur conserve la propriété du droit d'auteur et des droits moraux qui protège cette thèse. Ni la thèse ni des extraits substantiels de celle-ci ne doivent être imprimés ou autrement reproduits sans son autorisation.
In compliance with the Canadian Privacy Act some supporting forms may have been removed from this thesis.

While these forms may be included in the document page count, their removal does not represent any loss of content from the thesis.
Conformément à la loi canadienne sur la protection de la vie privée, quelques formulaires secondaires ont été enlevés de cette thèse.

Bien que ces formulaires aient inclus dans la pagination, if n'y aura aucun contenu manquant.

\section{Canadä}




\section{Abstract}

The problem of estimating the number of irreducible polynomials of degree $n$ over the finite field $\mathbb{F}_{q}$ with some given coefficients has been largely studied. We start by studying the number $N_{\gamma}(n, c, q)$ of irreducible polynomials of degree $n$ over $\mathbb{F}_{q}$ where the trace $\gamma$, which is the coefficient of $x^{n-1}$, and the constant term $c$ are given. Under some conditions on $n$ and $q$, we obtain bounds on the maximum of $N_{\gamma}(n, c, q)$ varying $c$ and $\gamma$. We show with concrete examples how our results improve previous known bounds. In addition, we improve upper and lower bounds of $N_{\gamma}(n, c, q)$ when $n=a(q-1)$ for nonzero constant term $c$ and nonzero trace $\gamma$. Moreover, we give a simple and explicit formula for the number $N(n, c, q)$ of irreducible polynomials over $\mathbb{F}_{q}$ of degree $n=q-1$ with prescribed primitive constant term $c$.

Moreover, several interesting results have been obtained for the number of irreducible polynomials of degree $n$ over $\mathbb{F}_{2}$ with some number of prescribed coefficients. As the second problem in this thesis, we study the number $N\left(n, t_{1}, t_{2}, t_{3}, t_{4}\right)$ of irreducible polynomials of even degree $n$ over the finite field $\mathbb{F}_{2}$ where the coefficients of the terms $x^{n-i}$ are given as $t_{i}$, for $i=1, \ldots, 4$. We give a formula for the number of elements $\beta \in \mathbb{F}_{2^{n}}$ where the $i^{\text {th }}$ trace of $\beta$ is given as $t_{i}$ and $i=1, \ldots, 4$, that we denote by $F\left(n, t_{1}, t_{2}, t_{3}, t_{4}\right)$. This formula is in terms of $N\left(n, t_{1}, t_{2}, t_{3}, t_{4}\right)$. We prove a generalization of Möbius inversion formula, and we use this generalization to give $N\left(n, t_{1}, t_{2}, t_{3}, t_{4}\right)$ in terms of the number $F\left(n, t_{1}, t_{2}, t_{3}, t_{4}\right)$. Then to give the exact value of $N\left(n, t_{1}, t_{2}, t_{3}, t_{4}\right)$, we study the number $F\left(n, t_{1}, t_{2}, t_{3}, t_{4}\right)$. This latest computation is quite challenging, so we present a good approximation for the number $N\left(n, t_{1}, t_{2}, t_{3}, t_{4}\right)$. 


\section{Acknowledgments}

I would like to thank my outstanding supervisor, Dr. Daniel Panario for his support, guidance, inspiration, and his great efforts to explain things clearly and simply. Throughout my thesis-writing period, he provided encouragement, sound advice, and lots of good ideas. For part of my thesis study, I had the chance to work with Dr. Qiang Wang which was a great experience for me. I would like to thank Dr. Wang for all his help, comments and advices for improving this thesis. In addition I wish to thank Dr. Brett Stevens for his great help during the time that we were checking the data by computer coding. I am thankful to all the defense committee members for reading the thesis and giving great comments.

I am grateful to the secretaries in the math department of Carleton University, for helping the department to run smoothly and for assisting me in many different ways. Also I am thankful to my friends at school who made these years easy and fun.

My late father was the person who gave me the initiative to pursue my graduate studies. I know that now he is happy and he is in my mind forever. Thank you mom and dad for everything you gave me. I wish to thank my entire extended family for providing a loving environment for me. My mother-in-law, my father-in-law and my sister-in-law were particularly supportive.

Finally I want to thank my family. The encouragement and support from my beloved wife Mina and our joyful daughter Aryana is a powerful source of inspiration and energy. Without their support and understanding it would have been impossible for me to finish this work. To them I dedicate this thesis.

Perhaps, I forgot someone, just in case: Thank you to whom it concerns! 
To my wife, Mina and my daughter, Aryana 


\section{Contents}

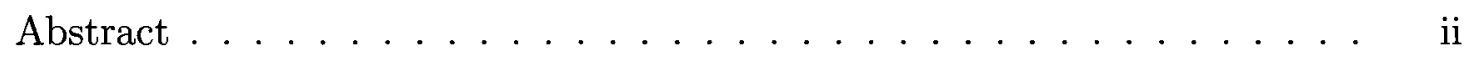

Acknowledgments ..................... iii

1 Introduction 1

1.1 A Summary of Preliminary Results . . . . . . . . . . . . 1

1.2 Contributions of This Thesis . . . . . . . . . . . 2

1.3 A Brief Description of This Thesis . . . . . . . . . . . . 3

2 Mathematical Background $\quad 5$

3 Generalizations of Möbius Inversion Formula 14

3.1 Background . . . . . . . . . . . . . . . . 15

3.2 Generalizing the Möbius Inversion Formula . . . . . . . . . . 16

4 The Number of Irreducible Polynomials with Given Trace and Norm

$\begin{array}{ll}\text { Terms } & 27\end{array}$

4.1 Background, Notation and Previous Results . . . . . . . . . 28

4.1 .1 The Structure of $D_{n} \ldots \ldots \ldots \ldots \ldots$

4.1.2 Fixed Constant Term With Different Traces . . . . . . . . 32

4.2 Our Bounds For $N_{\gamma}(n, c, q) \ldots \ldots \ldots \ldots \ldots \ldots \ldots$

4.3 The Special Case $n$ Being a Multiple of $q-1 \ldots \ldots \ldots 42$ 
5 The Number of Irreducible Polynomials of Even Degree $n$ Over $\mathbb{F}_{2}$ With the First Four Coefficients Prescribed

5.1 Preliminary Results and Background . . . . . . . . . . . . 54

5.2 The Formula For $F\left(n, t_{1}, t_{2}, t_{3}, t_{4}\right) \ldots \ldots \ldots \ldots \ldots \ldots \ldots \ldots \ldots$

5.3 The Formula For $N\left(n, t_{1}, t_{2}, t_{3}, t_{4}\right) \ldots \ldots \ldots \ldots \ldots$

6 An Attempt to Compute $F\left(n, t_{1}, t_{2}, t_{3}, t_{4}\right)$ Exactly $\quad 86$

6.1 Properties of Trace Functions . . . . . . . . . . . . . . 87

6.2 Cosets of $\mathbb{F}_{2^{l}}$ in $\mathbb{F}_{2^{n}} \ldots \ldots \ldots \ldots \ldots \ldots \ldots \ldots \ldots$

6.3 Traces in Different Categories . . . . . . . . . . . . . . . 108

7 Approximating the Number $N\left(n, t_{1}, t_{2}, t_{3}, t_{4}\right) \quad 118$

7.1 Estimation of $F\left(n, t_{1}, t_{2}, t_{3}, t_{4}\right) \ldots \ldots \ldots \ldots \ldots \ldots \ldots \ldots \ldots \ldots$

7.2 An Approximation of $N\left(n, t_{1}, t_{2}, t_{3}, t_{4}\right) \ldots \ldots \ldots \ldots$

7.3 Experimental Results . . . . . . . . . . . . . . . . . 133

8 Conclusions and Future Directions 139

8.1 Summary of Contributions . . . . . . . . . . . . . . . . . 139

8.2 Future Directions . . . . . . . . . . . . . . . . . . . 140

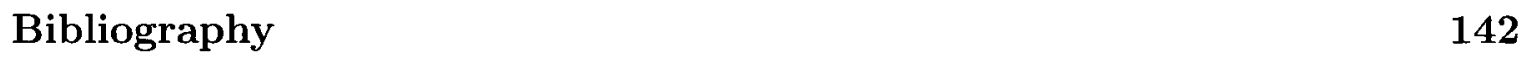




\section{Chapter 1}

\section{Introduction}

The origins of finite fields can be traced back to Gauss and Galois. The algebraic properties of finite fields were proved in the $19^{\text {th }}$ century. In the last 50 years there have been a revival in the interest in finite fields due mostly to their applications in cryptography, coding theory and communications. Irreducible polynomials play a central role in finite fields. Since they are the primes of the ring of polynomials over finite fields. This thesis centers around irreducible polynomials of special shape.

\subsection{A Summary of Preliminary Results}

The study of irreducible polynomials constitutes an important topic in finite fields. For example, they are used to represent the elements in extension fields. Let $q=p^{\omega}$, where $p$ is a prime. The problem of estimating the number of irreducible polynomials of degree $n$ over the finite field $\mathbb{F}_{q}$ with some coefficients prescribed to given values has been largely studied. Irreducible polynomials with many zero coefficients are important in efficient hardware implementation of feedback shift registers and finite field arithmetic (see [1]). 
Carlitz [2] and Kuz'min [12] give the number of irreducible polynomials with the first coefficient prescribed and the first two coefficients prescribed, respectively; see [3] for a similar result over $\mathbb{F}_{2}$, and [16] for more general results. Swan [21] gives the criteria under which trinomials over $\mathbb{F}_{2}$ are reducible, and Hsu [9] gives information on the distribution of irreducible polynomials with many coefficients prescribed to zero. Yucas [23] gives the number of irreducible polynomials with prescribed first or last coefficient. More recently, Kononen et al. [13] and Moisio [15] consider the number of irreducible polynomials with fixed trace and norm. Their approach is based on exponential sums and provide explicit results for some particular cases.

For even degree, Yucas and Mullen [24] consider the number of irreducible polynomials of degree $n$ over $\mathbb{F}_{2}$ where the first three coefficients, that is, the coefficients of $x^{n-1}, x^{n-2}$ and $x^{n-3}$, are prescribed. Fitzgerald and Yucas [7] consider the same problem, for odd degree $n$. The second part of this thesis centers in counting the number of irreducible polynomials of even degree $n$ over the finite field $\mathbb{F}_{2}$ with the first four coefficients being given. Our approach in the second part is similar to [24].

\subsection{Contributions of This Thesis}

We count the number of irreducible polynomials of degree $n$, over the finite field $\mathbb{F}_{q}$, where the trace and the constant term are given. There exists some bounds for the number of polynomials of this kind given in [22] and [16]. Under certain conditions on degree $n$, and characteristic $q$, we find better bounds for this number. Numerical results show that these bounds greatly improve the previous known bounds.

Next, we study the number of irreducible polynomials of even degree $n$ over the finite field $\mathbb{F}_{2}$ when the coefficients of the four terms $x^{n-1}, x^{n-2}, x^{n-3}$ and $x^{n-4}$ are prescribed. Möbius inversion formula is an important tool in counting the number of 
irreducible polynomials over the finite field $\mathbb{F}_{q}$. We give a generalization of Möbius inversion formula, that is used in our study of the number of irreducible polynomials of degree $n$ over $\mathbb{F}_{2}$ with given first four coefficients. We provide many intermediate results towards the exact computation of this number. Although we are not able to find its exact value, in the end of the thesis we give a good approximation of it. Experimental results show that the given approximation is very close to the exact value of the number of irreducible polynomials of even degree $n$ over $\mathbb{F}_{2}$ with first four prescribed coefficients

\subsection{A Brief Description of This Thesis}

In Chapter 2, we give the definitions and necessary background needed for studying this thesis.

In Chapter 3, we present a generalization of Möbius inversion formula to systems with $2^{j}$ arithmetic functions and $2^{j}$ equations, where $j \geq 2$. In the case $j=2$ we have a system of 4 equations in 4 functions, which is used in our study of the number of irreducible polynomials of degree $n$ over $\mathbb{F}_{2}$ where the coefficients of $x^{n-1}, x^{n-2}$, $x^{n-3}$ and $x^{n-4}$ are given; see Chapter 5. Parts of the results of Chapter 3 have been submitted [18].

We study in Chapter 4 the number of irreducible polynomials of degree $n$ over the finite field $\mathbb{F}_{q}$ with given trace and constant terms $\gamma, c \in \mathbb{F}_{q}$, respectively. This number is denoted by $N_{\gamma}(n, c, q)$. We find bounds on the maximum of $N_{\gamma}(n, c, q)$ under certain conditions on $q$ and $n$. Numerical examples show how our results improve existing bounds. Our results are particularly better when the degree $n$ is a multiple of $q-1$. We also find an explicit formula for the number of irreducible polynomials over $\mathbb{F}_{q}$ of degree $n=q-1$ with prescribed primitive constant term $c$, represented by $N(n, c, q)$. 
When $n=k(q-1)$ with $k>1$, we find a simple upper bound for $N(n, c, q)$. Finally, for $n=k(q-1)$ we improve upper and lower bounds of $N_{\gamma}(n, c, q)$, where trace $\gamma$ and constant term $c$ are nonzero. The results given in Chapter 4 have appeared in [17].

In Chapter 5 we study the number of irreducible polynomials of even degree $n$ over $\mathbb{F}_{2}$, where the four coefficients of $a_{n-1}, a_{n-2}, a_{n-3}$ and $a_{n-4}$ are prescribed. This is represented by $N\left(n, t_{1}, t_{2}, t_{3}, t_{4}\right)$ where $t_{i}=a_{n-i} \in \mathbb{F}_{2}$, for $i=1, \ldots, 4$. First we prove a formula for the number $F\left(n, t_{1}, t_{2}, t_{3}, t_{4}\right)$ of elements $\gamma \in \mathbb{F}_{2^{n}}$ where the $i^{\text {th }}$ trace of $\beta$ is $t_{i} \in \mathbb{F}_{2}$, and $i=1, \ldots, 4$. This formula is in terms of $N\left(n, t_{1}, t_{2}, t_{3}, t_{4}\right)$, and we use our generalization of Möbius inversion formula given in Chapter 3 to find a formula for $N\left(n, t_{1}, t_{2}, t_{3}, t_{4}\right)$ in terms of $F\left(n, t_{1}, t_{2}, t_{3}, t_{4}\right)$ as the main result of Chapter 5 .

To complete the computation of $N\left(n, t_{1}, t_{2}, t_{3}, t_{4}\right)$, in Chapter 6 we focus on the computation of the number $F\left(n, t_{1}, t_{2}, t_{3}, t_{4}\right)$. We let $n=4 l$, and $\gamma \in \mathbb{F}_{2^{n}}$. We consider different types of cosets $\gamma+\mathbb{F}_{2^{l}}$ of $\mathbb{F}_{2^{l}}$ in $\mathbb{F}_{2^{n}}$, and we divide them into different categories. Then we give the necessary and sufficient conditions for cosets in each category. Also, for different categories we compute $T_{i}(\gamma)$, the $i^{\text {th }}$ trace of $\gamma$, where $i=1, \ldots, 4$, and this leads to the computation of the number $F\left(n, t_{1}, t_{2}, t_{3}, t_{4}\right)$. It remains to compute the number of cases in each category that $T_{i}(\gamma)=0$ or $T_{i}(\gamma)=1$, for $i=1, \ldots, 4$.

Since the exact computation of $F\left(n, t_{1}, t_{2}, t_{3}, t_{4}\right)$ is not complete, we are not able to find the exact formula for $N\left(n, t_{1}, t_{2}, t_{3}, t_{4}\right)$. In Chapter 7 we give an estimate for the number $F\left(n, t_{1}, t_{2}, t_{3}, t_{4}\right)$, and using this estimate we find a good approximation for the number $N\left(n, t_{1}, t_{2}, t_{3}, t_{4}\right)$. Experimental results show that as $n$ grows, our approximation of $N\left(n, t_{1}, t_{2}, t_{3}, t_{4}\right)$ is very close to the exact value of $N\left(n, t_{1}, t_{2}, t_{3}, t_{4}\right)$.

We finish in Chapter 8 with some conclusions and future work. 


\section{Chapter 2}

\section{Mathematical Background}

We assume some basic knowledge of finite fields. The standard reference for these topics is [14]. We start with the definition of finite field.

Definition 1. A field is a set $\mathbb{F}$ together with two operations: + called addition, and called multiplication, which satisfy the following axioms: the set $\mathbb{F}$ is an abelian group under + with additive identity called zero and denoted by 0 ; the set $\mathbb{F}^{\times}$of all nonzero elements of $\mathbb{F}$ is also an abelian group under multiplication with multiplicative identity called one and denoted by 1 ; and multiplication distributes over addition. The field is finite if $\mathbb{F}$ has a finite number of elements, and the number of elements in $\mathbb{F}$ is called the order of $\mathbb{F}$.

In general, a finite field with $q$ element is denoted by $\mathbb{F}_{q}$ or $\operatorname{GF}(q)$.

Definition 2. A subfield of a finite field $\mathbb{F}_{q}$ is a subset $\mathbb{F}_{p} \subseteq \mathbb{F}_{q}$ which itself is a field under the operations of $\mathbb{F}_{q}$.

Let $\mathbb{F}_{q}$ be a finite field with $q=p^{n}$ elements. Then it is proved that every subfield of $\mathbb{F}_{q}$ has order $p^{m}$, where $m$ is a positive divisor of $n$. Conversely, if $m$ is a positive divisor of $n$, then there is exactly one subfield of $\mathbb{F}_{q}$ with $p^{m}$ elements. 
Definition 3. A field that contains no proper subfields is called a prime field. Therefore $F$ is a prime field if there is no subfield $K \subset F$.

The prime elements of $F[x]$ are called irreducible polynomials, and are defined as follow.

Definition 4. A polynomial $f \in F[x]$ is called irreducible over $F$ (or prime in $F[x]$ ) if $f$ has positive degree and $f=g h$ with $g, h \in F[x]$ implies that either $g$ or $h$ is a constant polynomial.

In brief, a polynomial $f$ of positive degree is irreducible over $F[x]$ if it has only trivial factorizations. The polynomial $f$ in $F[x]$ of positive degree is reducible over $F[x]$ if $f$ is not irreducible over $F$. The reducibility of a given polynomial $f$ over $F$ depends on the field $F$.

Irreducible polynomials are important for the structure of the ring $F[x]$ since polynomials in $F[x]$ can be written as products of irreducible polynomials in a unique way. For the polynomial $f \in F[x]$ it is known that the residue class $F[x] /(f)$ is a field if and only if $f$ is irreducible over $F$. If $f \in F[x]$ is irreducible, then any root of $f$ can be found in an extension field of $F$.

Some computations in finite fields and group theory are given using Euler's function which is defined here.

Definition 5. For $n \in \mathbb{N}$, Euler's function $\phi(n)$ is defined as the number of integers $k$ with $1 \leq k<n$ and $\operatorname{gcd}(k, n)=1$.

Let $m, n, s \in \mathbb{N}$ and $p$ be a prime. Then we have

(a) $\phi(p)=p-1$

(b) $\phi\left(p^{s}\right)=p^{s-1} \phi(p)=p^{s-1}(p-1)=p^{s}\left(1-\frac{1}{p}\right)$; 
(c) if $\operatorname{gcd}(m, n)=1$, then $\phi(m n)=\phi(m) \phi(n)$. In general if the integers $n_{1}, \ldots, n_{k}$ are such that $\operatorname{gcd}\left(n_{i}, n_{j}\right)=1$, for $i \neq j$, then $\phi\left(n_{1} \cdots n_{k}\right)=\phi\left(n_{1}\right) \cdots \phi\left(n_{k}\right) ;$

(d) if $n=p_{1}^{e_{1}} \ldots p_{k}^{e_{k}}$ is the prime factorization of $n$, then

$$
\phi(n)=n\left(1-\frac{1}{p_{1}}\right) \cdots\left(1-\frac{1}{p_{k}}\right)
$$

There are some concepts from group theory which we use and are defined here.

Definition 6. Let $G$ be a group, and $g$ be an element of $G$. The least positive integer $k$ such that $g^{k}=e$ is the order of $g$.

For any other integer $m$ if $g^{m}=e$, then $m$ is a multiple of $k$.

Definition 7. A multiplicative group is called cyclic, if there is an element $g \in G$ such that for any element $a \in G$ there is some $k$ such that $a=g^{k}$. The element $g$ is known as the generator of $G$, and we denote $G=\langle g\rangle$.

It is well known that for any finite field $\mathbb{F}_{q}$ the multiplicative group $\mathbb{F}_{q}^{\times}$of nonzero elements of $\mathbb{F}_{q}$ is cyclic; see [14]. In a cyclic group the elements which generate the group have special importance.

Definition 8. A generator of the cyclic group $\mathbb{F}_{q}^{\times}$is called a primitive element of the finite field $\mathbb{F}_{q}$.

It is proved that $\mathbb{F}_{q}$ contains $\phi(q-1)$ primitive elements, where $\phi$ is Euler's function.

In our method to study the number of irreducible polynomials, different results are proved using minimal polynomials which are defined as follow.

Definition 9. Let $\alpha \in \mathbb{F}_{q^{n}}$ be given. The minimal polynomial of $\alpha$ is the monic polynomial $f$, with coefficients in $\mathbb{F}_{q}$, of least degree such that $f(\alpha)=0$. 
The minimal polynomial $f$ of $\alpha$ is a unique monic irreducible polynomial over $\mathbb{F}_{q}$, and any other nonzero polynomial $g$ with $g(\alpha)=0$ is a multiple of $f$. The following theorem is proved in [8].

Theorem 10. If $f \in \mathbb{F}_{q}[x]$ and $\alpha$ is a root of $f$ in some extension field $\mathbb{F}_{q^{n}}$, then $f\left(x^{q}\right)=f(x)^{q}$ and $\alpha^{q}$ is also a root of $f$ in $\mathbb{F}_{q}[x]$.

Assuming that $\operatorname{Min}_{\alpha}$ is the minimal polynomial of $\alpha \in \mathbb{F}_{q^{n}}$, then $\alpha, \alpha^{q}, \alpha^{q^{2}}, \ldots$ are all roots of $\operatorname{Min}_{\alpha}$. This stops after, say $r$ terms, where $\alpha^{q^{r}}=\alpha$. Let $\gamma$ be a primitive element of $\mathbb{F}_{q^{n}}$. Then $\alpha=\gamma^{s}$, for some $s$. Hence $\alpha^{q^{r}}=\alpha$ if and only if $\gamma^{s q^{r}-s}=1$. Therefore we have $s q^{r} \equiv s\left(\bmod q^{n}-1\right)$. Based on this we define cyclotomic cosets which is an important concept in our work, and are used in different applications of irreducible polynomials; for example see [23].

Definition 11. The $q$-cyclotomic coset, or simply cyclotomic coset of $s$ modulo $q^{n}-1$ is denoted by $C_{s}$ and defined as the set $C_{s}=\left\{s, s q, \ldots, s q^{r}-1\right\}$, where $r$ is the smallest positive integer such that $s q^{r} \equiv s\left(\bmod q^{n}-1\right)$ and the computations in the set $C_{s}$ are $\bmod \left(q^{n}-1\right)$.

The sets $C_{s}$ partition the set $\left\{0,1, \ldots, q^{n}-2\right\}$ of integers into disjoint sets. Using primitive elements of a finite field and minimal polynomials we define primitive polynomials which are used in many applications about counting irreducible polynomials; see Cohen [5].

Definition 12. Let $\alpha \in \mathbb{F}_{q^{n}}$ be a primitive element. The polynomial $f \in \mathbb{F}_{q}[x]$ of degree $n$ is a primitive polynomial over $\mathbb{F}_{q}$ if $f$ is the minimal polynomial of $\alpha \in \mathbb{F}_{q^{n}}$.

Therefore the polynomial $f$ as a primitive polynomial over $\mathbb{F}_{q}$ of degree $n$ is a monic irreducible polynomial over $\mathbb{F}_{q}$ which has a root $\alpha$ in $\mathbb{F}_{q^{n}}$, and $\alpha$ is a generator of the finite field $\mathbb{F}_{q^{n}}$. 
Sometimes we view the finite extension $\mathbb{F}_{q^{n}}$ of the finite field $\mathbb{F}_{q}$ as a vector space over $\mathbb{F}_{q}$. Then $\mathbb{F}_{q^{n}}$ has dimension $n$ over $\mathbb{F}_{q}$. One special basis of $\mathbb{F}_{q^{n}}$ over $\mathbb{F}_{q}$ is defined using conjugates of an element $\alpha \in \mathbb{F}_{q^{n}}$.

Definition 13. Let $\mathbb{F}_{q^{n}}$ be an extension of $\mathbb{F}_{q}$ and let $\alpha \in \mathbb{F}_{q^{n}}$. Then the elements $\alpha, \alpha^{q}, \alpha^{q^{2}}, \ldots, \alpha^{q^{n-1}}$ are called the conjugates of $\alpha$ with respect to $\mathbb{F}_{q}$.

In the special case when $\alpha, \alpha^{q}, \ldots, \alpha^{q^{n-1}}$ are linearly independent, the basis defined by $\left\{\alpha, \alpha^{q}, \ldots, \alpha^{q^{n-1}}\right\}$ is called a normal basis of $\mathbb{F}_{q^{n}}$ over $\mathbb{F}_{q}$. Normal basis are important in many applications; see [20].

The conjugates of $\alpha \in \mathbb{F}_{q^{n}}$ with respect to $\mathbb{F}_{q}$ are distinct if and only if the minimal polynomial of $\alpha$ over $\mathbb{F}_{q}$ has degree $n$. Otherwise, the degree $d$ of this minimal polynomial is a proper divisor of $n$, and then the conjugates of $\alpha$ with respect to $\mathbb{F}_{q}$ are distinct elements $\alpha, \alpha^{q}, \ldots, \alpha^{q^{d-1}}$, each repeated $n / d$ times.

It is proved that, if $f \in \mathbb{F}_{q}$ is an irreducible polynomial of degree $n$ in $\mathbb{F}_{q}[x]$, then $f$ has a root $\alpha \in \mathbb{F}_{q^{n}}$. Moreover, all the roots of $f$ are simple and can be given by $n$ distinct elements $\alpha, \alpha^{q}, \ldots, \alpha^{q^{n-1}}$ of $\mathbb{F}_{q^{n}}$, which are conjugates of $\alpha$. Hence

$$
\begin{aligned}
f(x) & =x^{n}+a_{n-1} x^{n-1}+\cdots+a_{1} x+a_{0} \\
& =(x-\alpha)\left(x-\alpha^{2}\right) \cdots\left(x-\alpha^{q^{n-1}}\right) \\
& =x^{n}-\left(\alpha+\alpha^{q}+\cdots+\alpha^{q^{n-1}}\right) x^{n-1}+\cdots+(-1)^{n}\left(\alpha \alpha^{q} \ldots \alpha^{q^{n-1}}\right) .
\end{aligned}
$$

Therefore, finite fields can be used to factor irreducible polynomials. For any $\alpha \in \mathbb{F}_{q^{n}}$ the definitions of trace and norm of $\alpha$ are as follow.

Definition 14. Let $F=\mathbb{F}_{q^{n}}$ and $K=\mathbb{F}_{q}$. For $\alpha \in \mathbb{F}_{q^{n}}$, the trace $\operatorname{Tr}_{F / K}(\alpha)$ of $\alpha$ 
over $K$, or briefly $\operatorname{Tr}(\alpha)$ is defined by

$$
\operatorname{Tr}(\alpha)=\alpha+\alpha^{q}+\cdots+\alpha^{q^{n-1}}=\sum_{i=0}^{n-1} \alpha^{q^{i}}
$$

which is the sum of the conjugates of $\alpha$ with respect to $\mathbb{F}_{q}$. Similarly, the norm $N_{F / K}(\alpha)$ of $\alpha$ over $K$ is defined by

$$
N(\alpha)=\alpha \cdot \alpha^{q} \ldots \alpha^{q^{n-1}}=\alpha^{\frac{q^{n}-1}{q-1}} .
$$

In general the $k^{\text {th }}$ trace of $\alpha \in \mathbb{F}_{q^{n}}$, denoted by $T_{k}(\alpha)$ is defined by a $k$ sum,

$$
T_{k}(\alpha)=\sum_{0 \leq i_{1}<\cdots<i_{k} \leq n-1} \alpha^{i_{1}} \alpha^{i_{2}} \ldots \alpha^{i_{k}}
$$

The trace function $\operatorname{Tr}: \mathbb{F}_{q^{n}} \rightarrow \mathbb{F}_{q}$ has the following properties:

(i) $\operatorname{Tr}(\alpha+\beta)=\operatorname{Tr}(\alpha)+\operatorname{Tr}(\beta)$ for all $\alpha, \beta \in \mathbb{F}_{q^{n}}$;

(ii) $\operatorname{Tr}(k \alpha)=k \operatorname{Tr}(\alpha)$, for all $k \in \mathbb{F}_{q}$ and $\alpha \in \mathbb{F}_{q^{n}}$;

(iii) $\operatorname{Tr}(\alpha)=n \alpha$, for all $\alpha \in \mathbb{F}_{q}$;

(iv) $\operatorname{Tr}\left(\alpha^{q}\right)=\operatorname{Tr}(\alpha)$, for all $\alpha \in \mathbb{F}_{q^{n}}$;

(v) If $\mathbb{F}_{q^{n}}$ and $\mathbb{F}_{q}$ are viewed as vector spaces, then $\operatorname{Tr}$ can be considered as a linear transformation from $\mathbb{F}_{q^{n}}$ to $\mathbb{F}_{q}$.

The trace function $\mathbb{F}_{q^{n}} \rightarrow \mathbb{F}_{q}$ is a linear transformation that can be used to describe any other linear transformation from $\mathbb{F}_{q^{n}}$ onto $\mathbb{F}_{q}$, also known as linear functional. It is proved that a linear transformation (or linear functional) from $\mathbb{F}_{q^{n}}$ onto $\mathbb{F}_{q}$ is 
exactly the mapping $L_{\alpha}$, for some $\alpha \in \mathbb{F}_{q^{n}}$, defined as

$$
L_{\alpha}(\beta)=\operatorname{Tr}(\alpha \beta), \quad \text { for all } \beta \in \mathbb{F}_{q^{n}}
$$

Also, for all $\gamma, \delta \in \mathbb{F}_{q^{n}}$ we have $L_{\gamma}=L_{\delta}$, if and only if $\gamma=\delta$, and the linearity of the trace function implies that $L_{\gamma}+L_{\delta}=L_{\gamma+\delta}$.

If we compare the coefficients of the polynomial $f \in \mathbb{F}_{q}[x]$ given by

$$
\begin{aligned}
f(x) & =x^{n}+a_{n-1} x^{n-1}+\cdots+a_{1} x+a_{0} \\
& =x^{n}-\left(\alpha+\alpha^{q}+\cdots+\alpha^{q^{n-1}}\right) x^{n-1}+\cdots+(-1)^{n}\left(\alpha \cdot \alpha^{q} \ldots \alpha^{q^{n-1}}\right),
\end{aligned}
$$

and the definitions of trace and norm of $\alpha \in \mathbb{F}_{q^{n}}$, then we have $\operatorname{Tr}(\alpha)=-a_{n-1}$ and $a_{0}=(-1)^{n} N(\alpha)$, which implies $N(\alpha)=(-1)^{n} a_{0}$.

In a similar way, we define different traces of a polynomial $f \in \mathbb{F}_{q}[x]$.

Definition 15. Let $f \in \mathbb{F}_{q}[x]$ be a monic polynomial given by

$$
f(x)=x^{n}+a_{n-1} x^{n-1}+\cdots+a_{0} .
$$

Then the trace of $f$ written as $\operatorname{Tr}(f)$ is the coefficient of $x^{n-1}$, or $\operatorname{Tr}(f)=a_{n-1}$. In general, let $T_{k}(f)$, where $k=1, \ldots, n$ be the $k^{\text {th }}$ trace of $f$, which is defined as the coefficient of $x^{n-k}$ in $f$, that is, $T_{k}(f)=a_{n-k}$.

We observe that $T(f)$ is the coefficient of $f$, while $T(\alpha), \alpha \in \mathbb{F}_{q^{n}}$ is affected by a minus sign. Later we study the number of monic irreducible polynomials $f$ when some of the coefficients (or traces) are prescribed. Let $d$ be a positive integer. Then in our method, we need to have the coefficients of $f^{d}$ in terms of the coefficients of $f$. The following result, know as the Multinomial Theorem gives the connection 
between the coefficients of $f$ and $f^{d}$. Let $f$ be a given polynomial of degree $n$ and $d$ be a positive integer.

\section{Theorem 16. (Multinomial Theorem)}

Let $f$ be the linear function defined by

$$
f\left(x_{1}, \ldots, x_{m}\right)=x_{1}+x_{2}+\cdots+x_{m} .
$$

For the expansion of the polynomial $f^{d}$ we have the following formula

$$
\left(x_{1}+\cdots+x_{m}\right)^{d}=\sum_{d_{1}, \ldots, d_{m}} C\left(d ; d_{1}, d_{2}, \ldots, d_{m}\right) x_{1}{ }^{d_{1}} x_{2}{ }^{d_{2}} \ldots x_{m}{ }^{d_{m}},
$$

where in the sum $d_{1}+d_{2}+\cdots+d_{m}=d$, and

$$
C\left(d ; d_{1}, d_{2}, \ldots, d_{m}\right)=\frac{d !}{d_{1} ! d_{2} ! \ldots d_{m} !}
$$

In our studies, we are interested in some of the subsets of $\mathbb{F}_{q}$ called hyperplanes.

Definition 17. A hyperplane of the finite field $\mathbb{F}_{q^{n}}$ is an $(n-1)$-dimensional subspace of $\mathbb{F}_{q^{n}}$.

Cosets of a finite field are other well known subsets which are introduced in group theory and needed in our work.

Definition 18. Let $G$ be a multiplicative group and $H$ be a subgroup of $G$. For a fixed element $a \in G$ the left cosets of $G$ modulo $H$ denoted by aH are defined as

$$
a H=\{a h: h \in H\}
$$

The set of left cosets of $G$ modulo $H$ form a partition of $G$. For the additive group $G$ 
we define the left cosets of $G$ modulo $H$ as

$$
a+H=\{a+h: h \in H\} .
$$

Similarly, the right cosets of $G$ modulo $H$ have the form $H a=\{h a: h \in H\}$. If $G$ is an abelian group, then there is no distinction between left and right cosets modulo $H$.

Let $m$ and $n$ be two positive integers such that $m \mid n$. Then $\mathbb{F}_{q^{m}}$ is a subfield of $\mathbb{F}_{q^{n}}$. If $\gamma \in \mathbb{F}_{q^{n}}$ is given, then $\gamma+\mathbb{F}_{q^{m}}$ is a coset of $\mathbb{F}_{q^{n}}$. An element of the coset $\gamma+\mathbb{F}_{q^{m}}$ can be written as $\gamma+\beta$, where $\beta \in \mathbb{F}_{q^{m}}$.

Finally, we need the notion of isomorphism of groups.

Definition 19. Let $(G, *)$ and $(H, \circ)$ be two groups with given operations. A mapping $f: G \rightarrow H$ of the group $G$ into group $H$ is called a homomorphism of $G$ into $H$ if $f$ preserves the operation of $G$. This means for all $a, b \in G$ we have $f(a * b)=f(a) \circ f(b)$. If $f$ is one-to-one homomorphism of $G$ onto $H$, then $f$ is called an isomorphism and we say that $G$ and $H$ are isomorphic. 


\section{Chapter 3}

\section{Generalizations of Möbius}

\section{Inversion Formula}

Möbius inversion is an important formula in combinatorics and number theory. There exist many applications that use Möbius inversion. For example, the number of Lyndon words with given trace [19], and Dirichlet product [11] are given by means of Möbius inversion formula. In some counting problems in finite fields, a generalization of Möbius inversion formula is required to count the number of irreducible polynomial with some special form.

Cattell et al. [3] gives a generalization of Möbius inversion formula to find the number of irreducible polynomials over $\mathbb{F}_{2}$ with given coefficients of $x^{n-1}$ and $x^{n-2}$. For even degree $n$, Yucas and Mullen [24] consider the number of irreducible polynomials of degree $n$ over $\mathbb{F}_{2}$ with the first three coefficients prescribed (that is, the coefficients of $x^{n-1}, x^{n-2}$ and $x^{n-3}$ ). Fitzgerald and Yucas [7] consider the same problem for odd degree $n$. These problems required the same generalization of the Möbius inversion formula in [3].

In this chapter we provide a generalization of Möbius inversion formula to systems 
of $2^{j}$ equations in $2^{j}$ arithmetic functions, where $j \geq 2$. The smallest case of a system of 2 equations in 2 functions was treated in [3]. The case of 4 equations in 4 functions is used to study the number of irreducible polynomials of degree $n$ over $\mathbb{F}_{2}$ where the coefficients of $x^{n-1}, x^{n-2}, x^{n-3}$ and $x^{n-4}$ are given; see Chapter 5 .

Parts of the results presented in this chapter have been submitted [18].

\subsection{Background}

An arithmetic function is a function $f$ defined for all $n \in \mathbb{N}$. To count the number of monic irreducible polynomials in $\mathbb{F}_{q}[x]$ of fix degree, we need an arithmetic function, called Möbius function. It is denoted by $\mu$ and defined on $\mathbb{N}$ by the formula

$$
\mu(d)= \begin{cases}1 & \text { if } d=1 \\ 0 & \text { if } p^{2} \mid d \text { for some prime } p \\ (-1)^{r} & \text { if } d=p_{1} p_{2} \ldots p_{r} \text { where } p_{i} \text { are distinct primes. }\end{cases}
$$

For $n \in \mathbb{N}$, it is known [14] that

$$
\sum_{d \mid n} \mu(d)= \begin{cases}1 & \text { if } n=1 \\ 0 & \text { if } n>1\end{cases}
$$

Moreover, for any $n>1$ we have

$$
\mu(n)=-\sum_{\substack{d \mid n \\ d<n}} \mu(d) .
$$

The following is an important property of the Möbius function [14].

Theorem 20. (Möbius Inversion Formula) 
Let $f$ and $g$ be two arithmetic functions. Then

$$
f(n)=\sum_{d \mid n} g(d) \quad \text { for all } n \in \mathbb{N}
$$

if and only if

$$
g(n)=\sum_{d \mid n} \mu(d) f\left(\frac{n}{d}\right)=\sum_{d \mid n} \mu\left(\frac{n}{d}\right) f(d) \quad \text { for all } n \in \mathbb{N}
$$

The Möbius inversion formula is an important tool for counting the number of irreducible polynomials of a given degree $n$ over a finite field. Let $n \in \mathbb{N}$ and $N(n, q)$ be the number of monic irreducible polynomials in $\mathbb{F}_{q}[x]$ of degree $n$. Then we have

$$
q^{n}=\sum_{d \mid n} d N(n, q)
$$

and by Möbius inversion formula we have the following formula for $N(n, q)$ (see [14], for example):

$$
N(n, q)=\frac{1}{n} \sum_{d \mid n} \mu\left(\frac{n}{d}\right) q^{d}=\frac{1}{n} \sum_{d \mid n} \mu(d) q^{\frac{n}{d}}
$$

\subsection{Generalizing the Möbius Inversion Formula}

A generalization of Möbius inversion formula is given by Ruskey [3] to find the number of irreducible polynomials of degree $n$ over $\mathbb{F}_{2}$ where the coefficients of the terms $x^{n-1}$ and $x^{n-2}$ are given. The following theorem is proved in [3].

Theorem 21. Let $a \equiv b(\bmod 4)$ be shortened as $a \equiv b$. Also let $f, g, \alpha$, and $\beta$ be arithmetic functions. Then each of the functions $f$ and $g$ are connected to the other 
two functions $\alpha$ and $\beta$ as

$$
f(n)=\sum_{\substack{d \mid n \\ d \equiv 1}} \alpha(n / d)+\sum_{\substack{d \mid n \\ d \equiv 3}} \beta(n / d)
$$

and

$$
g(n)=\sum_{\substack{d \mid n \\ d \equiv 1}} \beta(n / d)+\sum_{\substack{d \mid n \\ d \equiv 3}} \alpha(n / d)
$$

if and only if

$$
\alpha(n)=\sum_{\substack{d \mid n \\ d \equiv 1}} \mu(d) f(n / d)+\sum_{\substack{d \mid n \\ d \equiv 3}} \mu(d) g(n / d)
$$

and

$$
\beta(n)=\sum_{\substack{d \mid n \\ d \equiv 1}} \mu(d) g(n / d)+\sum_{\substack{d \mid n \\ d \equiv 3}} \mu(d) f(n / d) .
$$

In [3] they use Theorem 21 to find $N\left(n, t_{1}, t_{2}, 2\right)$, which is the number of irreducible polynomials $f$ of degree $n$ over $\mathbb{F}_{2}$ where the coefficients of $x^{n-1}$ and $x^{n-2}$ are the numbers $t_{1}, t_{2} \in \mathbb{F}_{2}$.

Later in Chapter 5 we need a similar generalization to find the number of irreducible polynomials $f$ of degree $n$ over $\mathbb{F}_{2}$ with four given coefficients, denoted by $N\left(n, t_{1}, t_{2}, t_{3}, t_{4}, 2\right)$. As we comment later, our results apply to systems with $2^{j}$ equations and functions, though we only prove it here for the case of 4 equations and functions.

For a given statement $\mathrm{P}$ we let $[\mathrm{P}]=1$ if the statement $\mathrm{P}$ is true. If $\mathrm{P}$ is false then $[\mathrm{P}]=0$. Using this notation we prove the following lemma, which later we use to prove our generalization of Möbius inversion formula. 
Lemma 22. Let $a \equiv b(\bmod 8)$ be shortened to read $a \equiv b$. Then
(i) $\sum_{\substack{k|n \\ k| n \\ k \equiv 1}} \sum_{\substack{l \mid \frac{n}{k} \\ l \equiv 1}} a_{k, k l}=\sum_{\substack{m \mid n \\ m \equiv 1}} \sum_{\substack{k \mid m \\ k \equiv 1}} a_{k, m}$,
(ii) $\sum_{\substack{k \mid n \\ k \equiv 1}} \sum_{\substack{l \mid n \\ l=3}} a_{k, k l}=\sum_{\substack{m \mid n \\ m \equiv 3}} \sum_{\substack{k \mid m \\ k \equiv 1}} a_{k, m}$
(iii) $\sum_{\substack{k \mid n \\ k \equiv 1}} \sum_{\substack{l \mid n \\ k \equiv 5}} a_{k, k l}=\sum_{\substack{m \mid n \\ m \equiv 5}} \sum_{\substack{k \mid m \\ k \equiv 1}} a_{k, m}$
(iv) $\sum_{\substack{k \mid n \\ k \equiv 1}} \sum_{\substack{l \mid n \\ k=7}} a_{k, k l}=\sum_{\substack{m \mid n \\ m \equiv 7}} \sum_{\substack{k \mid m \\ k \equiv 1}} a_{k, m}$
(v) $\sum_{\substack{k \mid n \\ k=3}} \sum_{\substack{l \mid n \\ l=1 \\ l=1}} a_{k, k l}=\sum_{\substack{m \mid n \\ m \equiv 3}} \sum_{\substack{k \mid m \\ k \equiv 3}} a_{k, m}$
(vi) $\sum_{\substack{k \mid n \\ k \equiv 3}} \sum_{\substack{l, \frac{n}{k} \\ l=3}} a_{k, k l}=\sum_{\substack{m \mid n \\ m \equiv 1}} \sum_{\substack{k \mid m \\ k \equiv 3}} a_{k, m}$,
(vii) $\sum_{\substack{k \mid n \\ k=3}} \sum_{\substack{l \mid n \\ l=5}} a_{k, k l}=\sum_{\substack{m \mid n \\ m \equiv 7}} \sum_{\substack{k \mid m \\ k \equiv 3}} a_{k, m}$,
(viii) $\sum_{\substack{k \mid n \\ k \equiv 3}} \sum_{\substack{l \mid n \\ l=7 \\ l \equiv 7}} a_{k, k l}=\sum_{\substack{m \mid n \\ m \equiv 5}} \sum_{\substack{k \mid m \\ k \equiv 3}} a_{k, m}$
(ix) $\sum_{\substack{k \mid n \\ k \equiv 5}} \sum_{\substack{l \mid \frac{n}{k} \\ l \equiv 1}} a_{k, k l}=\sum_{\substack{m \mid n \\ m \equiv 5}} \sum_{\substack{k \mid m \\ k \equiv 5}} a_{k, m}$
(x) $\sum_{\substack{k \mid n \\ k \equiv 5}} \sum_{\substack{l\rangle n \\ l=3}} a_{k, k l}=\sum_{\substack{m \mid n \\ m \equiv 7}} \sum_{\substack{k \mid m \\ k \equiv 5}} a_{k, m}$
(xi) $\sum_{\substack{k \mid n \\ k \equiv 5}} \sum_{\substack{l \mid n \\ k=5}} a_{k, k l}=\sum_{\substack{m \mid n \\ m \equiv 1}} \sum_{\substack{k \mid m \\ k \equiv 5}} a_{k, m}$
(xii) $\sum_{\substack{k \mid n \\ k=5}} \sum_{\substack{l, n \\ l=7}} a_{k, k l}=\sum_{\substack{m \mid n \\ m \equiv 3}} \sum_{\substack{k \mid m \\ k \equiv 5}} a_{k, m}$
(xiii) $\sum_{\substack{k \mid n \\ k \equiv 7}} \sum_{\substack{l \mid n \\ l=1 \\ l \equiv 1}} a_{k, k l}=\sum_{\substack{m \mid n \\ m \equiv 7}} \sum_{\substack{k \mid m \\ k \equiv 7}} a_{k, m}$,
(ixv) $\sum_{\substack{k \mid n \\ k \equiv 7}} \sum_{\substack{l \mid n \\ k=3 \\ l \equiv 3}} a_{k, k l}=\sum_{\substack{m \mid n \\ m \equiv 5}} \sum_{\substack{k \mid m \\ k \equiv 7}} a_{k, m}$,
$(x v) \sum_{\substack{k \mid n \\ k \equiv 7}} \sum_{\substack{l \mid \frac{n}{k} \\ l \equiv 5}} a_{k, k l}=\sum_{\substack{m \mid n \\ m \equiv 3}} \sum_{\substack{k \mid m \\ k \equiv 7}} a_{k, m}$,
(xvi) $\sum_{\substack{k \mid n \\ k=7}} \sum_{\substack{l, n \\ k=7 \\ l \equiv 7}} a_{k, k l}=\sum_{\substack{m \mid n \\ m \equiv 1}} \sum_{\substack{k \mid m \\ k \equiv 7}} a_{k, m}$

Proof. We prove some parts only since the proofs of the other parts are similar. Suppose that $a \equiv b(\bmod 8)$ be written as $a \equiv b$. Let $n$ be a positive integer, and also $k$ and $m$ be divisors of $n$.

(ii) Since $k \equiv 1$ and $l \equiv 3$ then we have $k=8 k^{\prime}+1, l=8 l^{\prime}+3$, for some integers $k^{\prime}$ and $l^{\prime}$. Let $m=k l$. Then

$$
m=\left(8 k^{\prime}+1\right)\left(8 l^{\prime}+3\right)=8\left(8 k^{\prime} l^{\prime}+3 k^{\prime}+l^{\prime}\right)+3=8 m^{\prime}+3
$$


where $m^{\prime}=8 k^{\prime} l^{\prime}+3 k^{\prime}+l^{\prime}$. Therefore $m \equiv 3$, and we have

$$
[l \equiv 3][k \equiv 1]=[k l \equiv 3][k \equiv 1]=[m \equiv 3][k \equiv 1]
$$

This implies that

$$
\begin{aligned}
\sum_{\substack{k \mid n \\
k \equiv 1}} \sum_{\substack{l \mid \frac{n}{k} \\
l \equiv 3}} a_{k, k l} & =\sum_{j, m} \sum_{k, l>0} a_{k, k l}[n=j k][n / k=m l][l \equiv 3][k \equiv 1] \\
& =\sum_{m} \sum_{k, l>0} a_{k, k l}[n=m k l][l \equiv 3][k \equiv 1] .
\end{aligned}
$$

For the other side of Equation $(i i)$ we have

$$
\sum_{\substack{m \mid n \\ m \equiv 3}} \sum_{\substack{k \mid m \\ k \equiv 1}} a_{k, m}=\sum_{j, l} \sum_{k, m>0} a_{k, m}[n=j m][m=k l][m \equiv 3][k \equiv 1]
$$

In the latter sum we let $m=k l$ and use Equation (3.2). Then the sum can be given as

$$
\sum_{\substack{m \mid n \\ m \equiv 3}} \sum_{\substack{k \mid m \\ k \equiv 1}} a_{k, m}=\sum_{j} \sum_{k, l>0} a_{k, k l}[n=j k l][l \equiv 3][k \equiv 1],
$$

which proves Equation (ii), if in this sum we change $j$ by $m$.

(vi) Let $k \equiv 3$ and $l \equiv 3$. Then $k=8 k^{\prime}+3$ and $l=8 l^{\prime}+3$, for some integers $k^{\prime}$ and $l^{\prime}$. Again let $m=k l$. Then

$$
m=\left(8 k^{\prime}+3\right)\left(8 l^{\prime}+3\right)=8\left(8 k^{\prime} l^{\prime}+3 k^{\prime}+3 l^{\prime}+1\right)+1=8 m^{\prime}+1
$$


or $m \equiv 1$ and therefore $[l \equiv 3][k \equiv 3]=[m \equiv 1][k \equiv 3]$. Thus

$$
\begin{aligned}
\sum_{\substack{k \mid n \\
k \equiv 3}} \sum_{\substack{l \mid \frac{n}{k} \\
l \equiv 3}} a_{k, k l} & =\sum_{j, m} \sum_{k, l>0} a_{k, k l}[n=j k][n / k=m l][k \equiv 3][l \equiv 3] \\
& =\sum_{m} \sum_{k, l>0} a_{k, k l}[n=m k l][k \equiv 3][l \equiv 3] .
\end{aligned}
$$

Also,

$$
\begin{aligned}
\sum_{\substack{m \mid n \\
m \equiv 1}} \sum_{\substack{k \mid m \\
k \equiv 3}} a_{k, m} & =\sum_{j, l} \sum_{k, m>0} a_{k, k l}[n=j m][m=k l][m \equiv 1][k \equiv 3] \\
& =\sum_{m} \sum_{k, l>0} a_{k, k l}[n=m k l][k \equiv 3][l \equiv 3]
\end{aligned}
$$

which ends the proof of Equation $(v i)$.

In Lemma 22 the modulo is picked such that, if the values of the variables $k$ and $l$ are from the set $S=\left\{p_{1}, p_{2}, \ldots, p_{u}\right\}$, then the variable $m=k l$ is also in $S$. This means that the set of pairs $(k, l) \in S \times S$ is the same as the set of pairs $(m, k)$ in $S \times S$. For instance, if we use modulo 4 as in [3] and we let $S=\{1,3\}$, then the pairs $(k, l)$ in each of the sums can be $(1,1),(1,3),(3,1)$, or $(3,3)$. We have the exact same pairs for $(m, k)$. This implies that in Theorem 21 we can not change the moduli $d \equiv 1,3(\bmod 4)$. Any other moduli does not produce a valid result. Indeed for the case modulo 4 this is the only set $S$ where this is true.

In general, when we consider modulo $2^{j}$, the set $S$ is formed by the $2^{j-1}$ odd numbers smaller than $2^{j}$. Then a more general form of Lemma 22 can be given by the following lemma.

Lemma 23. Suppose $S=\left\{1,3, \ldots, 2^{j}-1\right\}$, and let $a \equiv b\left(\bmod 2^{j}\right)$ be shortened as 
$a \equiv b$. Assume that $r, s \in S$, such that $r s \equiv t$, for some $t \in S$. Then we have

$$
\sum_{\substack{k \mid n \\ k \equiv r}} \sum_{\substack{l \mid n \\ k \\ l \equiv s}} a_{k, k l}=\sum_{\substack{m \mid n \\ m \equiv t}} \sum_{\substack{k \mid m \\ k \equiv r}} a_{k, m}
$$

where for different $r, s \in S$ this gives $2^{2 j-2}$ different equations.

For example, if we let the modulo be 8 , then $S=\{1,3,5,7\}$ and as before the pairs $(k, l) \in S \times S$ are exactly the same as the pairs $(m, k) \in S \times S$. However, for systems with size $2^{j-1}$, where $j$ is bigger or equal to 3 we do have more variants. For example, when we consider modulo 8 we need four functions $(A, B, C, D)$ where each one is given by an equation in terms of four other functions $(\alpha, \beta, \gamma, \delta)$. In these equations, the ordering of $(\alpha, \beta, \gamma, \delta)$ is only relevant as long as each function appears exactly once per equation and once per sum modulo 8 . We return to this issue after the proof of the theorem. The ordering $(\alpha, \beta, \gamma, \delta)$ in Theorem 24 matches the order needed in Chapter 5 .

We use Lemma 22 to prove the main result of this chapter.

\section{Theorem 24. (Generalized Möbius Inversion formula)}

Let $A(n), B(n), C(n), D(n), \alpha(n), \beta(n), \gamma(n)$, and $\delta(n)$ be functions defined on $\mathbb{N}$. Then

$$
\begin{aligned}
& A(n)=\sum_{\substack{d \mid n \\
d \equiv=1}} \alpha\left(\frac{n}{d}\right)+\sum_{\substack{d \mid n \\
d \equiv 3}} \beta\left(\frac{n}{d}\right)+\sum_{\substack{d \mid n \\
d \equiv 5}} \gamma\left(\frac{n}{d}\right)+\sum_{\substack{d \mid n \\
d \equiv 7}} \delta\left(\frac{n}{d}\right), \\
& B(n)=\sum_{\substack{d \mid n \\
d \equiv 1}} \beta\left(\frac{n}{d}\right)+\sum_{\substack{d \mid n \\
d \equiv 3}} \alpha\left(\frac{n}{d}\right)+\sum_{\substack{d \mid n \\
d \equiv 5}} \delta\left(\frac{n}{d}\right)+\sum_{\substack{d \mid n \\
d \equiv 7}} \gamma\left(\frac{n}{d}\right), \\
& C(n)=\sum_{\substack{d \mid n \\
d \equiv 1}} \gamma\left(\frac{n}{d}\right)+\sum_{\substack{d \mid n \\
d \equiv 3}} \delta\left(\frac{n}{d}\right)+\sum_{\substack{d \mid n \\
d \equiv 5}} \alpha\left(\frac{n}{d}\right)+\sum_{\substack{d \mid n \\
d \equiv}} \beta\left(\frac{n}{d}\right), \\
& D(n)=\sum_{\substack{d \mid n \\
d \equiv 7}} \delta\left(\frac{n}{d}\right)+\sum_{\substack{d \mid n \\
d \equiv 3}} \gamma\left(\frac{n}{d}\right)+\sum_{\substack{d \mid n \\
d \equiv 5}} \beta\left(\frac{n}{d}\right)+\sum_{\substack{d \mid n \\
d \equiv}} \alpha\left(\frac{n}{d}\right),
\end{aligned}
$$


if and only if

$$
\begin{aligned}
& \alpha(n)=\sum_{\substack{d \mid n \\
d \equiv 1}} \mu(d) A\left(\frac{n}{d}\right)+\sum_{\substack{d \mid n \\
d \equiv 3}} \mu(d) B\left(\frac{n}{d}\right)+\sum_{\substack{d \mid n \\
d \equiv 5}} \mu(d) C\left(\frac{n}{d}\right)+\sum_{\substack{d \mid n \\
d \equiv 7}} \mu(d) D\left(\frac{n}{d}\right), \\
& \beta(n)=\sum_{\substack{d \mid n \\
d \equiv 1}} \mu(d) B\left(\frac{n}{d}\right)+\sum_{\substack{d \mid n \\
d \equiv 3}} \mu(d) A\left(\frac{n}{d}\right)+\sum_{\substack{d \mid n \\
d \equiv 5}} \mu(d) D\left(\frac{n}{d}\right)+\sum_{\substack{d \mid n \\
d \equiv 7}} \mu(d) C\left(\frac{n}{d}\right), \\
& \gamma(n)=\sum_{\substack{d \mid n \\
d \equiv 1}} \mu(d) C\left(\frac{n}{d}\right)+\sum_{\substack{d \mid n \\
d \equiv 3}} \mu(d) D\left(\frac{n}{d}\right)+\sum_{\substack{d \mid n \\
d \equiv 5}} \mu(d) A\left(\frac{n}{d}\right)+\sum_{\substack{d \mid n \\
d \equiv 7}} \mu(d) B\left(\frac{n}{d}\right), \\
& \delta(n)=\sum_{\substack{d \mid n \\
d \equiv 1}} \mu(d) D\left(\frac{n}{d}\right)+\sum_{\substack{d \mid n \\
d \equiv 3}} \mu(d) C\left(\frac{n}{d}\right)+\sum_{\substack{d \mid n \\
d \equiv 5}} \mu(d) B\left(\frac{n}{d}\right)+\sum_{\substack{d \mid n \\
d \equiv 7}} \mu(d) A\left(\frac{n}{d}\right) .
\end{aligned}
$$

Proof. Suppose that $A(n), B(n), C(n), D(n), \alpha(n), \beta(n), \gamma(n)$, and $\delta(n)$ are functions on $\mathbb{N}$ such that

$$
\begin{gathered}
\alpha(n)=\sum_{\substack{d \mid n \\
d \equiv 1}} \mu(d) A\left(\frac{n}{d}\right)+\sum_{\substack{d \mid n \\
d \equiv 3}} \mu(d) B\left(\frac{n}{d}\right)+\sum_{\substack{d \mid n \\
d \equiv 5}} \mu(d) C\left(\frac{n}{d}\right)+\sum_{\substack{d \mid n \\
d \equiv 7}} \mu(d) D\left(\frac{n}{d}\right), \\
\beta(n)=\sum_{\substack{d \mid n \\
d \equiv 1}} \mu(d) B\left(\frac{n}{d}\right)+\sum_{\substack{d \mid n \\
d \equiv 3}} \mu(d) A\left(\frac{n}{d}\right)+\sum_{\substack{d \mid n \\
d \equiv 5}} \mu(d) D\left(\frac{n}{d}\right)+\sum_{\substack{d \mid n \\
d \equiv 7}} \mu(d) C\left(\frac{n}{d}\right), \\
\gamma(n)=\sum_{\substack{d \mid n \\
d \equiv 1}} \mu(d) C\left(\frac{n}{d}\right)+\sum_{\substack{d \mid n \\
d \equiv 3}} \mu(d) D\left(\frac{n}{d}\right)+\sum_{\substack{d \mid n \\
d \equiv 5}} \mu(d) A\left(\frac{n}{d}\right)+\sum_{\substack{d \mid n \\
d \equiv 7}} \mu(d) B\left(\frac{n}{d}\right), \\
\delta(n)=\sum_{\substack{d \mid n \\
d \equiv 1}} \mu(d) D\left(\frac{n}{d}\right)+\sum_{\substack{d \mid n \\
d \equiv 3}} \mu(d) C\left(\frac{n}{d}\right)+\sum_{\substack{d \mid n \\
d \equiv 5}} \mu(d) B\left(\frac{n}{d}\right)+\sum_{\substack{d \mid n \\
d \equiv}} \mu(d) A\left(\frac{n}{d}\right) .
\end{gathered}
$$

Let us define

$$
f(n)=\sum_{\substack{d \mid n \\ d \equiv 1}} \alpha\left(\frac{n}{d}\right)+\sum_{\substack{d \mid n \\ d \equiv 3}} \beta\left(\frac{n}{d}\right)+\sum_{\substack{d \mid n \\ d \equiv 5}} \gamma\left(\frac{n}{d}\right)+\sum_{\substack{d \mid n \\ d \equiv 7}} \delta\left(\frac{n}{d}\right)
$$

We want to show that $f(n)=A(n)$, for all $n \in \mathbb{N}$. By the definitions of the functions $\alpha, \beta, \gamma$ and $\delta$, the function $f(n)$ can be given as

$$
\sum_{\substack{d|n \\ d| n}}\left(\sum_{\substack{c \mid n \\ d=1}} \mu(c) A\left(\frac{n / d}{c}\right)+\sum_{\substack{c \mid n \\ c \equiv 1 \\ c \equiv 3}} \mu(c) B\left(\frac{n / d}{c}\right)+\sum_{\substack{c \mid n \\ d=0 \\ c \equiv 5}} \mu(c) C\left(\frac{n / d}{c}\right)+\sum_{\substack{c|n \\ c| n \\ c \equiv 7}} \mu(c) D\left(\frac{n / d}{c}\right)\right)
$$




$$
\begin{aligned}
& +\sum_{\substack{d \mid n \\
d \equiv 3}}\left(\sum_{\substack{c \mid \frac{n}{d} \\
c \equiv 1}} \mu(c) B\left(\frac{n / d}{c}\right)+\sum_{\substack{c \mid \frac{n}{d} \\
c \equiv 3}} \mu(c) A\left(\frac{n / d}{c}\right)+\sum_{\substack{c \mid \frac{n}{d} \\
c \equiv 5}} \mu(c) D\left(\frac{n / d}{c}\right)+\sum_{\substack{c \mid \frac{n}{d} \\
c \equiv 7}} \mu(c) C\left(\frac{n / d}{c}\right)\right) \\
& +\sum_{\substack{d \mid n \\
d \equiv 5}}\left(\sum_{\substack{c \mid \frac{n}{d} \\
c \equiv 1}} \mu(c) C\left(\frac{n / d}{c}\right)+\sum_{\substack{c \mid \frac{n}{d} \\
c \equiv 3}} \mu(c) D\left(\frac{n / d}{c}\right)+\sum_{\substack{c \mid \frac{n}{d} \\
c \equiv 5}} \mu(c) A\left(\frac{n / d}{c}\right)+\sum_{\substack{c \mid \frac{n}{d} \\
c \equiv 7}} \mu(c) B\left(\frac{n / d}{c}\right)\right) \\
& +\sum_{\substack{d \mid n \\
d \equiv 7}}\left(\sum_{\substack{c \mid \frac{n}{d} \\
c \equiv 1}} \mu(c) D\left(\frac{n / d}{c}\right)+\sum_{\substack{c \mid \frac{n}{d} \\
c \equiv 3}} \mu(c) C\left(\frac{n / d}{c}\right)+\sum_{\substack{c \mid \frac{n}{d} \\
c \equiv 5}} \mu(c) B\left(\frac{n / d}{c}\right)+\sum_{\substack{c \mid \frac{n}{d} \\
c \equiv 7}} \mu(c) A\left(\frac{n / d}{c}\right)\right) .
\end{aligned}
$$

So $f(n)$ has 16 double sums. Suppose that $c d=m$ and so $d \mid m$. Since $d \mid n$ and $c \mid \frac{n}{d}$ we have $c d \mid n$, or $m \mid n$. So $f(n)$ changes to

$$
\begin{aligned}
& f(n)=\sum_{\substack{m \mid n \\
m \equiv 1}} \sum_{\substack{d \mid m \\
d \equiv 1}} \mu\left(\frac{m}{d}\right) A\left(\frac{n}{m}\right)+\sum_{\substack{m \mid n \\
m \equiv 3}} \sum_{\substack{d \mid m \\
d \equiv 1}} \mu\left(\frac{m}{d}\right) B\left(\frac{n}{m}\right)+\sum_{\substack{m \mid n \\
m \equiv 5}} \sum_{\substack{d \mid m \\
d \equiv 1}} \mu\left(\frac{m}{d}\right) C\left(\frac{n}{m}\right) \\
& +\sum_{\substack{m \mid n \\
m \triangleq 7}} \sum_{\substack{d \mid m \\
d \equiv 1}} \mu\left(\frac{m}{d}\right) D\left(\frac{n}{m}\right)+\sum_{\substack{m \mid n \\
m \equiv 3}} \sum_{\substack{d \mid m \\
d \equiv 3}} \mu\left(\frac{m}{d}\right) B\left(\frac{n}{m}\right)+\sum_{\substack{m \mid n \\
m \equiv 1}} \sum_{\substack{d \mid m \\
d \equiv 3}} \mu\left(\frac{m}{d}\right) A\left(\frac{n}{m}\right) \\
& +\sum_{\substack{m \mid n \\
m \equiv 7}} \sum_{\substack{d \mid m \\
d \equiv 3}} \mu\left(\frac{m}{d}\right) D\left(\frac{n}{m}\right)+\sum_{\substack{m \mid n \\
m \equiv 5}} \sum_{\substack{d \mid m \\
d \equiv 3}} \mu\left(\frac{m}{d}\right) C\left(\frac{n}{m}\right)+\sum_{\substack{m \mid n \\
m \equiv 5}} \sum_{\substack{d \mid m \\
d \equiv 5}} \mu\left(\frac{m}{d}\right) C\left(\frac{n}{m}\right) \\
& +\sum_{\substack{m \mid n \\
m \equiv 7}} \sum_{\substack{d \mid m \\
d \equiv 5}} \mu\left(\frac{m}{d}\right) D\left(\frac{n}{m}\right)+\sum_{\substack{m \mid n \\
m \equiv 1}} \sum_{\substack{d \mid m \\
d \equiv 5}} \mu\left(\frac{m}{d}\right) A\left(\frac{n}{m}\right)+\sum_{\substack{m \mid n \\
m \equiv 3}} \sum_{\substack{d \mid m \\
d \equiv 5}} \mu\left(\frac{m}{d}\right) B\left(\frac{n}{m}\right) \\
& +\sum_{\substack{m \mid n \\
m \equiv 7}} \sum_{\substack{d \mid m \\
d \equiv 7}} \mu\left(\frac{m}{d}\right) D\left(\frac{n}{m}\right)+\sum_{\substack{m \mid n \\
m \equiv 5}} \sum_{\substack{d \mid m \\
d \equiv 7}} \mu\left(\frac{m}{d}\right) C\left(\frac{n}{m}\right)+\sum_{\substack{m \mid n \\
m \equiv 3}} \sum_{\substack{d \mid m \\
d \equiv 7}} \mu\left(\frac{m}{d}\right) B\left(\frac{n}{m}\right) \\
& +\sum_{\substack{m|n \\
m| n}} \sum_{d \mid m} \mu\left(\frac{m}{d}\right) A\left(\frac{n}{m}\right) \\
& =\sum_{\substack{m \mid n \\
m \equiv 1}} A\left(\frac{n}{m}\right) \sum_{\substack{d \mid m \\
d \equiv 1}} \mu\left(\frac{m}{d}\right)+\sum_{\substack{m \mid n \\
m \equiv 3}} B\left(\frac{n}{m}\right) \sum_{\substack{d \mid m \\
d \equiv 1}} \mu\left(\frac{m}{d}\right)+\sum_{\substack{m \mid n \\
m \equiv 5}} C\left(\frac{n}{m}\right) \sum_{\substack{d \mid m \\
d \equiv 1}} \mu\left(\frac{m}{d}\right) \\
& +\sum_{\substack{m \mid n \\
m \equiv 7}} D\left(\frac{n}{m}\right) \sum_{\substack{d \mid m \\
d \equiv 1}} \mu\left(\frac{m}{d}\right)+\sum_{\substack{m \mid n \\
m \equiv 3}} B\left(\frac{n}{m}\right) \sum_{\substack{d \mid m \\
d \equiv 3}} \mu\left(\frac{m}{d}\right)+\sum_{\substack{m \mid n \\
m \equiv 1}} A\left(\frac{n}{m}\right) \sum_{\substack{d \mid m \\
d \equiv 3}} \mu\left(\frac{m}{d}\right) \\
& +\sum_{\substack{m \mid n \\
m \equiv 7}} D\left(\frac{n}{m}\right) \sum_{\substack{d \mid m \\
d \equiv 3}} \mu\left(\frac{m}{d}\right)+\sum_{\substack{m \mid n \\
m \equiv 5}} C\left(\frac{n}{m}\right) \sum_{\substack{d \mid m \\
d \equiv 3}} \mu\left(\frac{m}{d}\right)+\sum_{\substack{m \mid n \\
m \equiv 5}} C\left(\frac{n}{m}\right) \sum_{\substack{d \mid m \\
d \equiv 5}} \mu\left(\frac{m}{d}\right) \\
& +\sum_{\substack{m \mid n \\
m \equiv 7}} D\left(\frac{n}{m}\right) \sum_{\substack{d \mid m \\
d \equiv 5}} \mu\left(\frac{m}{d}\right)+\sum_{\substack{m \mid n \\
m \equiv 1}} A\left(\frac{n}{m}\right) \sum_{\substack{d \mid m \\
d \equiv 5}} \mu\left(\frac{m}{d}\right)+\sum_{\substack{m \mid n \\
m \equiv 3}} B\left(\frac{n}{m}\right) \sum_{\substack{d \mid m \\
d \equiv 5}} \mu\left(\frac{m}{d}\right)
\end{aligned}
$$




$$
\begin{aligned}
& +\sum_{\substack{m \mid n \\
m \equiv 7}} D\left(\frac{n}{m}\right) \sum_{\substack{d \mid m \\
d \equiv 7}} \mu\left(\frac{m}{d}\right)+\sum_{\substack{m \mid n \\
m \equiv 5}} C\left(\frac{n}{m}\right) \sum_{\substack{d \mid m \\
d \equiv 7}} \mu\left(\frac{m}{d}\right)+\sum_{\substack{m \mid n \\
m \equiv 3}} B\left(\frac{n}{m}\right) \sum_{\substack{d \mid m \\
d \equiv 7}} \mu\left(\frac{m}{d}\right) \\
& +\sum_{\substack{m \mid n \\
m \equiv 1}} A\left(\frac{n}{m}\right) \sum_{\substack{d \mid m \\
d \equiv 7}} \mu\left(\frac{m}{d}\right) \\
& =\sum_{\substack{m \mid n \\
m \equiv 1}} A\left(\frac{n}{m}\right) \sum_{\substack{d \mid m \\
d \text { odd }}} \mu\left(\frac{m}{d}\right)+\sum_{\substack{m \mid n \\
m \equiv 3}} B\left(\frac{n}{m}\right) \sum_{\substack{d \mid m \\
d \text { odd }}} \mu\left(\frac{m}{d}\right) \sum_{\substack{m \mid n \\
m \equiv 5}} C\left(\frac{n}{m}\right) \sum_{\substack{d \mid m \\
d \text { odd }}} \mu\left(\frac{m}{d}\right) \\
& +\sum_{\substack{m \mid n \\
m \equiv 7}} D\left(\frac{n}{m}\right) \sum_{\substack{d \mid m \\
d \text { odd }}} \mu\left(\frac{m}{d}\right) .
\end{aligned}
$$

It is clear that in each of these double sums $m$ is an odd number. Now $d \mid m$, and so $d$ is an odd number. Therefore the internal sums simplify to

$$
\sum_{\substack{d \mid m \\
d \text { odd }}} \mu\left(\frac{m}{d}\right)=\sum_{d \mid m} \mu\left(\frac{m}{d}\right)=\left\{\begin{array}{cc}
1 & \text { if } m=1 \\
0 & \text { otherwise }
\end{array}\right.
$$

We have that $m=1$ is only possible for the first double sum in $f(n)$. Thus, those other double sums are zero. Moreover $m=1$ implies $d=1$, and therefore $f(n)$ simplifies to

$$
f(n)=\sum_{\substack{m \mid n \\ m \equiv 1}} A\left(\frac{n}{m}\right) \sum_{\substack{d \mid m \\ d \text { odd }}} \mu\left(\frac{m}{d}\right)=A(n)
$$

In a similar way we can prove $B(n), C(n)$ and $D(n)$.

To prove the other side of the if and only if statement, let

$$
\theta(n)=\sum_{\substack{d \mid n \\ d \equiv 1}} \mu(d) A\left(\frac{n}{d}\right)+\sum_{\substack{d \mid n \\ d \equiv 3}} \mu(d) B\left(\frac{n}{d}\right)+\sum_{\substack{d \mid n \\ d \equiv 5}} \mu(d) C\left(\frac{n}{d}\right)+\sum_{\substack{d \mid n \\ d \equiv 7}} \mu(d) D\left(\frac{n}{d}\right)
$$

We show that $\theta(n)=\alpha(n)$, for all $n \in \mathbb{N}$. If in $\theta(n)$ we substitute for $A\left(\frac{n}{d}\right), B\left(\frac{n}{d}\right)$, 
$C\left(\frac{n}{d}\right)$ and $D\left(\frac{n}{d}\right)$ then with similar simplifications as above we have

$$
\begin{aligned}
\theta(n) & =\sum_{\substack{m \mid n \\
m \equiv 1}} \alpha\left(\frac{n}{m}\right) \sum_{\substack{d|m \\
d| m}} \mu\left(\frac{m}{d}\right)+\sum_{\substack{m \mid n \\
m \equiv 3}} \beta\left(\frac{n}{m}\right) \sum_{\substack{d|m \\
d| m}} \mu\left(\frac{m}{d}\right) \\
& +\sum_{\substack{m \mid n \\
m \equiv 5}} \gamma\left(\frac{n}{m}\right) \sum_{\substack{d \mid m \\
d \text { odd }}} \mu\left(\frac{m}{d}\right)+\sum_{\substack{m \mid n \\
m \equiv 7}} \delta\left(\frac{n}{m}\right) \sum_{\substack{d \mid m \\
d \text { odd }}} \mu\left(\frac{m}{d}\right) .
\end{aligned}
$$

Again, $m$ and $d$ are both odd numbers which means that $\theta(n)$ can be given as

$$
\begin{aligned}
\theta(n) & =\sum_{\substack{m \mid n \\
m \equiv 1}} \alpha\left(\frac{n}{m}\right) \sum_{d \mid m} \mu\left(\frac{m}{d}\right)+\sum_{\substack{m \mid n \\
m \equiv 3}} \beta\left(\frac{n}{m}\right) \sum_{d \mid m} \mu\left(\frac{m}{d}\right) \\
& +\sum_{\substack{m \mid n \\
m \equiv 5}} \gamma\left(\frac{n}{m}\right) \sum_{d \mid m} \mu\left(\frac{m}{d}\right)+\sum_{\substack{m \mid n \\
m \equiv 7}} \delta\left(\frac{n}{m}\right) \sum_{d \mid m} \mu\left(\frac{m}{d}\right) .
\end{aligned}
$$

Therefore if $m=1$ then just the first sum is nonzero, and the other three are zero. Moreover $m=1$ implies $d=1$, and so $\theta$ simplifies to

$$
\theta(n)=\alpha(n / 1) \mu(1)=\alpha(n) \cdot 1=\alpha(n) .
$$

Similarly we prove the results for $\beta(n), \gamma(n)$ and $\delta(n)$.

Let $j>3$ be an integer. With similar arguments as in Theorem 24 we obtain a generalization to systems of $2^{j-1}$ equations in $2^{j-1}$ functions. In this case we have that $S$ is the set of odd numbers $S=\left\{1,3, \ldots, 2^{j}-1\right\}$. One can check that the set of pairs $(k, l)$ in $S \times S$ is the set of pairs $(m, k)$ in $S \times S$. Hence, we have a similar result with $2^{j-1}$ sums running over the odd divisors of $n$ modulo $2^{j}$. Moreover, the order of the $2^{j-1}$ functions in each equation is irrelevant, provided that each function appears once per equation and once per different moduli in the sums, exactly as in the case $j=3$ in Theorem 24 . 
Theorem 25. Suppose that $j>2$ be a given integer. Let $f_{i}$ and $g_{i}$ be functions defined on $\mathbb{N}$, where $i \in\left\{1,2,3, \ldots, 2^{j-1}\right\}$. Then

$$
\begin{gathered}
f_{1}(n)=\sum_{\substack{d \mid n \\
d \equiv 1}} g_{1}\left(\frac{n}{d}\right)+\sum_{\substack{d \mid n \\
d \equiv 3}} g_{2}\left(\frac{n}{d}\right)+\cdots+\sum_{\substack{d \mid n \\
d \equiv 2^{j}-1}} g_{2^{j-1}}\left(\frac{n}{d}\right), \\
f_{2}(n)=\sum_{\substack{d \mid n \\
d \equiv 1}} g_{2}\left(\frac{n}{d}\right)+\sum_{\substack{d \mid n \\
d \equiv 3}} g_{1}\left(\frac{n}{d}\right)+\cdots+\sum_{\substack{d \mid n \\
d \equiv 2^{j}-1}} g_{2^{j-1}-1}\left(\frac{n}{d}\right),
\end{gathered}
$$

and so on until

$$
f_{2^{j-1}}(n)=\sum_{\substack{d \mid n \\ d \equiv 1}} g_{2^{j-1}}\left(\frac{n}{d}\right)+\sum_{\substack{d \mid n \\ d \equiv 3}} g_{2^{j-1}-1}\left(\frac{n}{d}\right)+\cdots+\sum_{\substack{d \mid n \\ d \equiv 2^{j}-1}} g_{1}\left(\frac{n}{d}\right)
$$

if and only if

$$
\begin{aligned}
& g_{1}(n)=\sum_{\substack{d \mid n \\
d \equiv 1}} \mu(d) f_{1}\left(\frac{n}{d}\right)+\sum_{\substack{d \mid n \\
d \equiv 3}} \mu(d) f_{2}\left(\frac{n}{d}\right)+\cdots+\sum_{\substack{d \mid n \\
d \equiv 2^{j}-1}} \mu(d) f_{2^{j-1}}\left(\frac{n}{d}\right), \\
& g_{2}(n)=\sum_{\substack{d \mid n \\
d \equiv 1}} \mu(d) f_{2}\left(\frac{n}{d}\right)+\sum_{\substack{d \mid n \\
d \equiv 3}} \mu(d) f_{1}\left(\frac{n}{d}\right)+\cdots+\sum_{\substack{d \mid n \\
d \equiv 2^{j}-1}} \mu(d) f_{2^{j-1}-1}\left(\frac{n}{d}\right),
\end{aligned}
$$

and so on until

$$
g_{2^{j-1}}(n)=\sum_{\substack{d \mid n \\ d \equiv 1}} \mu(d) f_{2^{j-1}}\left(\frac{n}{d}\right)+\sum_{\substack{d \mid n \\ d \equiv 3}} \mu(d) f_{2^{j-1}-1}\left(\frac{n}{d}\right)+\cdots+\sum_{\substack{d \mid n \\ d \equiv 2^{j}-1}} \mu(d) f_{1}\left(\frac{n}{d}\right)
$$




\section{Chapter 4}

\section{The Number of Irreducible}

\section{Polynomials with Given Trace and}

\section{Norm Terms}

In this chapter we study the number $N_{\gamma}(n, c, q)$ of irreducible polynomials $f \in \mathbb{F}_{q}[x]$ of degree $n$ where the trace $\gamma$ and the constant term $c$ are given. In Section 4.1 we review the required background and fix the notation for this chapter. The main results of this chapter are given in Sections 4.2 and 4.3. We obtain bounds on the maximum of $N_{\gamma}(n, c, q)$ under certain conditions on $q$ and $n$ (Theorem 31). We show with concrete examples how our results improve previous bounds.

Our results are particularly better when the degree $n$ is a multiple of $q-1$. We treat this case in Section 4.3. We give a simple and explicit formula for the number $N(n, c, q)$ of irreducible polynomials over $\mathbb{F}_{q}$ of degree $n=q-1$ with prescribed primitive constant term $c$, and a simple upper bound of it for $n=a(q-1)$ with $a>1$ (Theorem 34). Finally, we obtain improved upper and lower bounds of $N_{\gamma}(n, c, q)$ with $n=a(q-1)$ and nonzero trace $\gamma$ and nonzero constant term $c$ (Theorems 38 
and 39 , respectively).

Parts of the results given in this chapter have appeared in [17].

\subsection{Background, Notation and Previous Results}

Counting the number of irreducible polynomials of degree $n$ over the finite field $\mathbb{F}_{q}$ with some given coefficients has been studied by several mathematicians. The number of irreducible polynomials with prescribed trace is given by Carlitz [2]. When the first two coefficients are fixed, that is the coefficients of $x^{n-1}$ and $x^{n-2}$, the result is given by Kuz'min [12]. If the polynomial is over $\mathbb{F}_{2}$, then Cattell et al [3] has a similar result; see [16] for more general results. Swan [21] gives the criteria under which trinomials over $\mathbb{F}_{2}$ are reducible, and Hsu [9] gives information on the distribution of irreducible polynomials with many coefficients prescribed to zero. Yucas [23] gives the number of irreducible polynomials with prescribed first or last coefficient. Other results are given in Chapter 1.

The number of irreducible polynomials of degree $n$ and trace $\gamma$ over $\mathbb{F}_{q}$ is denoted by $N_{\gamma}(n, q)$. For given $p$ and $m$, we say that $m$ is $p$ free, if $p \nmid m$. Suppose that $n=p^{\kappa} \psi$, where $\psi$ is pfree. Then for $\gamma \neq 0$, Corollary 2.7 of [23] proves that the number $N_{\gamma}(n, q)$ can be given as

$$
N_{\gamma}(n, q)=\frac{1}{n q} \sum_{d \mid \psi} \mu(d) q^{\frac{n}{d}}
$$

where $\mu$ represents the Möbius function. If $n=p^{\kappa} \psi$, then using $\kappa$ we introduce a variable $\varepsilon$, as

$$
\varepsilon= \begin{cases}1 & \text { if } \kappa>0 \\ 0 & \text { if } \kappa=0\end{cases}
$$


Let $n=p^{\kappa} \psi$, where $\psi$ is pfree. Then for $\gamma=0$, Corollary 2.8 of [23] gives $N_{0}(n, q)$ as

$$
N_{0}(n, q)=\frac{1}{n q} \sum_{d \mid \psi} \mu(d) q^{\frac{n}{d}}-\frac{\varepsilon}{n} \sum_{d \mid \psi} \mu(d) q^{\frac{n}{p d}}
$$

We use $N(n, c, q)$ for the number of irreducible polynomials of degree $n$ and constant term $c$ over $\mathbb{F}_{q}$. Let

$$
D_{n}=\left\{r: r \mid q^{n}-1, r \nmid q^{m}-1 \text { for } m<n\right\} .
$$

For each $r \in D_{n}$, let $r=m_{r} d_{r}$, where $d_{r}=\operatorname{gcd}\left(r, \frac{q^{n}-1}{q-1}\right)$. It is easy to see that $m_{r} \mid q-1$. Suppose that the order of the constant $c$ is $\rho$. In [23] it is shown that the number $N(n, c, q)$ can be found as

$$
N(n, c, q)=\frac{1}{n \phi(\rho)} \sum_{\substack{r \in D_{n} \\ m_{r}=\rho}} \phi(r)
$$

where $\phi$ denotes Euler's function. In this sum for each $r \in D_{n}$ the number $m_{r}$ is fixed as $\rho=\operatorname{ord}(c)$. If both trace $\gamma$ and a nonzero constant $c$ are prescribed, Carlitz [2] obtained an asymptotic formula, as $n \rightarrow \infty$,

$$
N_{\gamma}(n, c, q)=\frac{q^{n}-1}{n q(q-1)}+O\left(q^{\frac{n}{2}}\right)
$$

Using a bijection $f(x) \mapsto c^{-1} x^{n} f\left(\frac{1}{x}\right), N_{\gamma}(n, c, q)$ equals the number of irreducible polynomials of degree $n$ in the arithmetic progression $\left\{a x+c+g(x) x^{2} \mid g(x) \in \mathbb{F}_{q}[x]\right\}$, where $a=-\gamma c^{-1}$. Applying a general asymptotic bound on the number of primes on an arithmetic progression, Moisio [15] pointed out the following improvement of 
Equation (4.2), as $n \rightarrow \infty$,

$$
N_{\gamma}(n, c, q)=\frac{q^{n-1}}{n(q-1)}+O\left(\frac{q^{\frac{n}{2}}}{n}\right)
$$

For the estimation of the error term, Wan [22] established the following effective bound

$$
\left|N_{\gamma}(n, c, q)-\frac{q^{n-1}}{n(q-1)}\right| \leq \frac{3}{n} q^{\frac{n}{2}}
$$

Recently, this bound was improved by Moisio [15] by considering two separate cases whether $\gamma$ is zero or not. He obtained for nonzero $\gamma$,

$$
\left|N_{\gamma}(n, c, q)-\frac{q^{n}-1}{n q(q-1)}\right|<\frac{2}{q-1} q^{\frac{n}{2}}
$$

and for zero trace

$$
\left|N_{0}(n, c, q)-\frac{q^{n-1}-1}{n(q-1)}\right|<\frac{2}{q-1} q^{\frac{n}{2}} .
$$

The focus of this chapter is in the study of $N_{\gamma}(n, c, q)$, where $\gamma$ and $c$ are given.

\subsubsection{The Structure of $D_{n}$}

For a better understanding of $N_{\gamma}(n, c, q)$, where $1 \leq \gamma \leq q-1$, we need to know the structure of the set

$$
D_{n}=\left\{r: r \mid q^{n}-1, r \nmid q^{m}-1 \text { for } m<n\right\} .
$$

Let us assume that we have the prime factorization $q-1=p_{1}^{g_{1}} \ldots p_{k}^{g_{k}}$, such that $p_{1}, \ldots, p_{k}$ are distinct prime factors, and $g_{i} \geq 1$ for $1 \leq i \leq k$. Similarly, we let

$$
q^{n}-1=p_{1}^{e_{1}} \ldots p_{k}^{e_{k}} p_{k+1}^{e_{k+1}} \ldots p_{t}^{e_{t}}
$$


where $e_{i} \geq g_{i} \geq 1$, for $1 \leq i \leq k$, and $e_{i} \geq 1$, for $k+1 \leq i \leq t$. Let us define $S_{1}=\{1, \ldots, k\}$, and $S_{2}=\{k+1, \ldots, t\}$. We have the following lemma.

Lemma 26. For each $r \mid q^{n}-1$ where $r=m_{r} d_{r}$, with $d_{r}=\operatorname{gcd}\left(r, \frac{q^{n}-1}{q-1}\right)$ and $m_{r} \mid q-1$ there exists a positive integer $R$ such that $r=\frac{q^{n}-1}{R}$, and $\operatorname{gcd}(R, q-1)=\frac{q-1}{m_{r}}$.

Proof. Since $r \mid q^{n}-1$, there exists $R$ such that $r=\frac{q^{n}-1}{R}$. Since $r=m_{r} d_{r}$ with $m_{r} \mid q-1$ and $d_{r}=\operatorname{gcd}\left(r, \frac{q^{n}-1}{q-1}\right)$, there exist integers $T$ and $V$, such that $q-1=m_{r} T$, and $\frac{q^{n}-1}{q-1}=d_{r} V$. Therefore,

$$
\begin{aligned}
\operatorname{gcd}(R, q-1) & =\operatorname{gcd}\left(\frac{q^{n}-1}{m_{r} d_{r}}, q-1\right)=\frac{q-1}{m_{r}} \operatorname{gcd}\left(\frac{q^{n}-1}{d_{r}(q-1)}, m_{r}\right) \\
& =\frac{q-1}{m_{r}} \operatorname{gcd}\left(V, m_{r}\right) .
\end{aligned}
$$

Moreover $d_{r}=\operatorname{gcd}\left(r, \frac{q^{n}-1}{q-1}\right)=\operatorname{gcd}\left(m_{r} d_{r}, d_{r} V\right)=d_{r} \operatorname{gcd}\left(m_{r}, V\right)$. Hence, we get $\operatorname{gcd}\left(m_{r}, V\right)=1$, and the result follows.

Clearly from

$$
r=\frac{q^{n}-1}{R}=\frac{(q-1)\left(q^{n-1}+q^{n-2}+\cdots+q+1\right)}{R}
$$

we have $R \mid(q-1)\left(q^{n-1}+q^{n-2}+\cdots+q+1\right)$. Since $\operatorname{gcd}(R, q-1)=\frac{q-1}{m_{r}}$, then in terms of the $\operatorname{gcd}(R, q-1)$ we can consider two cases:

Case 1: If $\operatorname{gcd}(R, q-1)=\frac{q-1}{m_{r}}=1$, then $m_{r}=q-1$, and all the factors of $R$ are from $q^{n-1}+q^{n-2}+\cdots+q+1$, and not from $q-1$. Then $r=p_{1}^{e_{1}} \ldots p_{k}^{e_{k}} p_{k+1}^{f_{k+1}} \ldots p_{t}^{f_{t}}$, where $0 \leq f_{i} \leq e_{i}$, for $i \in S_{2}$.

Case 2: If $\operatorname{gcd}(R, q-1)>1$, then $m_{r}<q-1$ and there exist some common primes between $R$ and $q-1$. Then let $r$ be given by

$$
r=p_{1}^{f_{1}} p_{2}^{f_{2}} \ldots p_{k}^{f_{k}} p_{k+1}^{f_{k+1}} \ldots p_{t}^{f_{t}}
$$


where $f_{i} \leq e_{i}$ for all $i \in S_{1} \cup S_{2}$, and let us assume that the factorization of $q^{m}-1$ is

$$
q^{m}-1=p_{1}^{h_{m, 1}} \ldots p_{k}^{h_{m, k}} p_{k+1}^{h_{m, k+1}} \ldots p_{t}^{h_{m, t}} p_{t+1}^{h_{m, t+1}} \ldots p_{l}^{h_{m, l}}
$$

where $h_{m, i} \geq g_{i}$, for $i \in S_{1}$, and $h_{m, i} \geq 0$, for $i \in S_{2}$. Also for all $i=t+1, \ldots, l$, we have $h_{m, i} \geq 1$.

Now let us consider the structure of $D_{n}$. For the above $r$ to be in $D_{n}, r$ must not be a divisor of $q^{m}-1$ for any $m \leq n-1$. To separate the two cases, we represent the elements of $D_{n}$ by $r$ and $r^{\prime}$ where $r=\frac{q^{n}-1}{R}$, with $\operatorname{gcd}(R, q-1)=1$, and $r^{\prime}=\frac{q^{n}-1}{R^{\prime}}$ such that $\operatorname{gcd}\left(R^{\prime}, q-1\right)>1$ respectively. Let $p_{1}^{f_{1}} \ldots p_{k}^{f_{k}} p_{k+1}^{f_{k+1}} \ldots p_{t}^{f_{t}}$ be any $r$ or $r^{\prime}$ from the set $D_{n}$. Since $r$ and $r^{\prime}$ are not divisors of $q^{m}-1$, for $m \leq n-1$, we have the following conditions

1. $f_{i} \leq e_{i}$ for all $i \in S_{1} \cup S_{2}$;

2.(a) $f_{j}=e_{j}$, for all $j \in S_{1}$, and $r=p_{1}^{e_{1}} \ldots p_{k}^{e_{k}} p_{k+1}^{f_{k+1}} \ldots p_{t}^{f_{t}} \nmid q^{m}-1$, for $m \leq n-1$; or

2.(b) There exist $\delta \in S_{1}$ such that $f_{\delta}<e_{\delta}$. Then since for all $m \leq n-1$ we have $p_{1}^{f_{1}} \ldots p_{k}^{f_{k}} p_{k+1}^{f_{k+1}} \ldots p_{t}^{f_{t}} \nmid q^{m}-1$, by considering (4.3) as the factorization of $q^{m}-1$, there exists $j \in S_{1} \cup S_{2}$ such that $f_{j}>h_{m, j}$.

\subsubsection{Fixed Constant Term With Different Traces}

Let $c \in \mathbb{F}_{q}^{\times}$be a fixed nonzero constant. We study the number of irreducible polynomials of degree $n$ and constant term $c$ for different values of the trace coefficient.

Lemma 27. Let $\gamma$ and $\delta$ be two nonzero traces. If $c$ is a constant from $\mathbb{F}_{q}^{\times}$, then

$$
N_{\gamma}(n, c, q)=N_{\delta}\left(n, c\left(\frac{\delta}{\gamma}\right)^{n}, q\right)
$$


Proof. Suppose $\gamma$ and $\delta$ are two nonzero traces in $\mathbb{F}_{q}$, and let $P_{\gamma}(n, c, q)$ denote the set of all irreducible polynomials of degree $n$, trace $\gamma$ and constant term $c$ over the finite field $\mathbb{F}_{q}$. We show that there exists a one-to-one correspondence between $P_{\gamma}(n, c, q)$ and $P_{\delta}\left(n, c\left(\frac{\delta}{\gamma}\right)^{n}, q\right)$. For this we consider the mapping used in Lemma 2.1 of [23]. Namely, let the mapping $\varphi: P_{\gamma}(n, c, q) \rightarrow P_{\delta}\left(n, c\left(\frac{\delta}{\gamma}\right)^{n}, q\right)$ be defined by

$$
\varphi(f(x))=\left(\frac{\delta}{\gamma}\right)^{n} f\left(\frac{\gamma}{\delta} x\right)
$$

If $f$ is an irreducible polynomial, then $g(x)=\left(\frac{\delta}{\gamma}\right)^{n} f\left(\frac{\gamma}{\delta} x\right)$ is irreducible too. To prove this, we can use a contradiction argument. If $g$ is reducible, then $f\left(\frac{\gamma}{\delta} x\right)$ is reducible, and we can assume that $f\left(\frac{\gamma}{\delta} x\right)=h(x) k(x)$, where $\operatorname{deg}(h)+\operatorname{deg}(k)=n=\operatorname{deg}(f)$. This means at least one of the factors $h$ or $k$ is in $f$, while $f$ is irreducible.

Now we show that $\phi$ is well-defined and it is a bijection. Let $f \in P_{\gamma}(n, c, q)$ be a fixed polynomial. We show that there exists some $g \in P_{\delta}\left(n, c\left(\frac{\delta}{\gamma}\right)^{n}, q\right)$ such that $\varphi(f)=g$. The polynomial $f \in P_{\gamma}(n, c, q)$ can be written as

$$
f(x)=a_{n} x^{n}+\gamma x^{n-1}+a_{n-2} x^{n-2}+\cdots+c .
$$

Then applying the mapping $\varphi$ we have

$$
\begin{aligned}
\varphi(f(x)) & =\left(\frac{\delta}{\gamma}\right)^{n} f\left(\frac{\gamma}{\delta} x\right) \\
& =\left(\frac{\delta}{\gamma}\right)^{n}\left(a_{n}\left(\frac{\gamma}{\delta} x\right)^{n}+\gamma\left(\frac{\gamma}{\delta} x\right)^{n-1}+\cdots+c\right) \\
& =a_{n}\left(\frac{\delta}{\gamma}\right)^{n}\left(\frac{\gamma}{\delta}\right)^{n} x^{n}+\gamma\left(\frac{\delta}{\gamma}\right)^{n}\left(\frac{\gamma}{\delta}\right)^{n-1} x^{n-1}+\cdots+c\left(\frac{\delta}{\gamma}\right)^{n} \\
& =a_{n} x^{n}+\delta x^{n-1}+\cdots+c\left(\frac{\delta}{\gamma}\right)^{n}=g(x),
\end{aligned}
$$


which $g$ is a polynomial in $P_{\delta}\left(n, c\left(\frac{\delta}{\gamma}\right)^{n}, q\right)$. So $\varphi$ is a well-defined map. Also $\varphi$ is onto. To prove this, let $g$ be any polynomial in the set $P_{\delta}\left(n, c\left(\frac{\delta}{\gamma}\right)^{n}, q\right)$. We show that there exist $f \in P_{\gamma}(n, c, q)$ such that $\varphi(f)=g$, where $\gamma$ is any nonzero element in $\mathbb{F}_{q}$. The polynomial $g \in P_{\delta}\left(n, c\left(\frac{\delta}{\gamma}\right)^{n}, q\right)$ can be considered as

$$
g(x)=b_{n} x^{n}+\delta x^{n-1}+\cdots+c\left(\frac{\delta}{\gamma}\right)^{n}
$$

We can rewrite $g$ as,

$$
\begin{aligned}
g(x) & =\left(\frac{\delta}{\gamma}\right)^{n}\left(b_{n}\left(\frac{\gamma}{\delta}\right)^{n} x^{n}+\gamma\left(\frac{\gamma}{\delta}\right)^{n-1} x^{n-1}+\cdots+c\right) \\
& =\left(\frac{\delta}{\gamma}\right)^{n}\left(b_{n}\left(\frac{\gamma}{\delta} x\right)^{n}+\gamma\left(\frac{\gamma}{\delta} x\right)^{n-1}+\cdots+c\right)
\end{aligned}
$$

Now let $f(x)=b_{n} x^{n}+\gamma x^{n-1}+\cdots+c$, where $\gamma$ is a nonzero element in $\mathbb{F}_{q}^{\times}$. Then $f \in P_{\gamma}(n, c, q)$ and

$$
g(x)=\left(\frac{\delta}{\gamma}\right)^{n} f\left(\frac{\gamma}{\delta} x\right)=\varphi(f(x)) .
$$

Therefore $\varphi$ is onto. Finally we show that $\varphi$ is a one-to-one. Let $f_{1}$ and $f_{2}$ be two distinct polynomials in $P_{\gamma}(n, c, q)$. We show that $\varphi\left(f_{1}\right) \neq \varphi\left(f_{2}\right)$. Let $f_{1}$ and $f_{2}$ be given as

$$
f_{1}(x)=f_{1, n} x^{n}+\gamma x^{n-1}+\cdots+f_{1, i} x^{i}+\cdots+c
$$

and

$$
f_{2}(x)=f_{2, n} x^{n}+\gamma x^{n-1}+\cdots+f_{2, i} x^{i}+\cdots+c
$$

Since $f_{1} \neq f_{2}$, without loss of generality suppose that

$$
f_{1, i} \neq f_{2, i}, \quad \text { for some } \quad 1 \leq i \leq n-2 .
$$


Then applying $\phi$ on $f_{1}$ and $f_{2}$ we have

$$
\begin{aligned}
& \varphi\left(f_{1}(x)\right)=\left(\frac{\delta}{\gamma}\right)^{n}\left(f_{1, n} x^{n}+\delta x^{n-1}+\cdots+f_{1, i}\left(\frac{\delta}{\gamma}\right)^{n-i} x^{i}+\cdots+c\right), \\
& \varphi\left(f_{2}(x)\right)=\left(\frac{\delta}{\gamma}\right)^{n}\left(f_{2, n} x^{n}+\delta x^{n-1}+\cdots+f_{2, i}\left(\frac{\delta}{\gamma}\right)^{n-i} x^{i}+\cdots+c\right) .
\end{aligned}
$$

Therefore the coefficients of $x^{i}$ in $\varphi\left(f_{1}(x)\right)$ and $\varphi\left(f_{2}(x)\right)$ are different, which implies $\varphi\left(f_{1}\right) \neq \varphi\left(f_{1}\right)$ and $\phi$ one-to-one. Since $\phi$ is a bijection between $P_{\gamma}(n, c, q)$ and $P_{\gamma}\left(n, c\left(\frac{\delta}{\gamma}\right)^{n}, q\right)$, then $\left|P_{\gamma}(n, c, q)\right|=\left|P_{\gamma}\left(n, c\left(\frac{\delta}{\gamma}\right)^{n}, q\right)\right|$ which concludes that

$$
N_{\gamma}(n, c, q)=N_{\delta}\left(n, c\left(\frac{\delta}{\gamma}\right)^{n}, q\right)
$$

Let $\mathbb{F}_{q}=\left\{a_{0}=0, a_{1}=1, a_{2}, \ldots, a_{q-1}\right\}$. The following table gives the number of irreducible polynomials of degree $n$ with given trace and constant term.

Table 4.1: Distribution of polynomials of degree $n$ over a finite field $\mathbb{F}_{q}$.

\begin{tabular}{|c|c|c|c|c|c|c|}
\hline \multicolumn{1}{|c|}{ Cons } & $a_{1}$ & $\cdots$ & $a_{j}$ & $\cdots$ & $a_{q-1}$ & Row Total \\
\hline$a_{0}$ & $y_{0,1}$ & $\cdots$ & $y_{0, j}$ & $\cdots$ & $y_{0, q-1}$ & $N_{0}(n, q)$ \\
\hline$a_{1}$ & $x_{1,1}$ & $\cdots$ & $x_{1, j}$ & $\cdots$ & $x_{1, q-1}$ & $N_{1}(n, q)$ \\
\hline$\vdots$ & $\vdots$ & & $\vdots$ & & $\vdots$ & $\vdots$ \\
\hline$a_{i}$ & $x_{i, 1}$ & $\cdots$ & $x_{i, j}$ & $\cdots$ & $x_{i, q-1}$ & $N_{i}(n, q)$ \\
\hline$\vdots$ & $\vdots$ & & $\vdots$ & & $\vdots$ & $\vdots$ \\
\hline$a_{q-1}$ & $x_{q-1,1}$ & $\cdots$ & $x_{q-1, j}$ & $\cdots$ & $x_{q-1, q-1}$ & $N_{q-1}(n, q)$ \\
\hline Column Total & $N(n, 1, q)$ & $\cdots$ & $N(n, j, q)$ & $\cdots$ & $N(n, q-1, q)$ & $N(n, q)$ \\
\hline
\end{tabular}

Abusing notation, if $c=a_{j} \in \mathbb{F}_{q}^{\times}$, for some $j \in\{1,2, \ldots, q-1\}$, then we denote $N(n, c, q)$ by $N(n, j, q)$. Also for $\gamma=a_{i}$, where $i \in\{0,1, \ldots, q-1\}$, we use $N_{i}(n, q)$ for $N_{\gamma}(n, q)$. Moreover $N_{\gamma}(n, c, q)=N_{i}(n, j, q)$, where $0 \leq i, j \leq q-1$, and $j \neq 0$. 
For simplicity, we use notations $x_{i, j}$ for $N_{i}(n, j, q)$ where $1 \leq i, j \leq q-1$, and $y_{0, j}$ for $N_{0}(n, j, q)$ where $1 \leq j \leq q-1$.

For any $n$, we know that $c\left(\frac{\delta}{\gamma}\right)^{n}=c^{\prime}$ is a constant in $\mathbb{F}_{q}$. Clearly by Lemma 27 , we have $N_{\gamma}(n, c, q)=N_{\delta}\left(n, c^{\prime}, q\right)$, which implies that for any nonzero traces $\gamma=a_{i}$ and $\delta=a_{k}$ the numbers on the row $a_{k}$ are a permutation of the numbers on the row $a_{i}$, where $1 \leq i, k \leq q-1$. If we consider any column which is related to a constant $c=a_{j}$ then we have an equation of the form

$$
y_{0, j}+\sum_{i=1}^{q-1} x_{i, j}=N(n, j, q)
$$

Also in column $a_{j}$ we know that some entries $x_{i, j}$ could be repeated. Let us define $R_{j}=\{1,2, \ldots, k\}$ as the set of indices $i$ in the column $a_{j}$ such that no $x_{i, j}$ is repeated. Clearly $R_{j} \subseteq\{1,2, \ldots, q-1\}$, and if in the column $a_{j}$ there is no repeated entry, then $R_{j}=\{1,2, \ldots, q-1\}$. Let $A_{i, j}$ represent the number of times $x_{i, j}$ appears in the entries of column $a_{j}$. Then by Equation (4.4), for each column $a_{j}$, we have

$$
y_{0, j}+\sum_{i \in R_{j}} A_{i, j} x_{i, j}=N(n, j, q)
$$

The last column of Table 4.1 gives the total number of polynomials in each row, and the last row gives the total number of polynomials in each column. By Equation (4.1) we have

$$
N(n, q)=N_{0}(n, q)+\sum_{i=1}^{q-1} N_{i}(n, q)=N_{0}(n, q)+(q-1) N_{1}(n, q)
$$

Next we study $x_{i, j}, y_{0, j}$ and $N(n, j, q)$. 


\subsection{Our Bounds For $N_{\gamma}(n, c, q)$}

Let $\gamma=a_{i} \in \mathbb{F}_{q}$, and $c=a_{j} \in \mathbb{F}_{q}^{\times}$be any given elements, where $0 \leq i \leq q-1$, and $1 \leq j \leq q-1$. In Theorem 5.1 of [22], bounds for the number $x_{i, j}$ are given as

$$
\left|x_{i, j}-\frac{q^{n-1}}{n(q-1)}\right| \leq \frac{3}{n} q^{\frac{n}{2}}
$$

In [15], better bounds for $x_{i, j}$ are given by considering different cases for the trace. If the trace is zero, from Corollary 3.4 of [15], then we have the following bounds for $y_{0, j}$

$$
\left|y_{0, j}-\frac{q^{n-1}-1}{n(q-1)}\right| \leq \frac{s-1}{n} q^{\frac{n-2}{2}}+\frac{q^{\frac{n}{2}}-1}{q-1}<\frac{2}{q-1} q^{\frac{n}{2}}
$$

where $s=\operatorname{gcd}(n, q-1)$. For a nonzero trace $\gamma=a_{i}$ we have $i>0$. By Corollary 4.3 of [15], we have the following bounds for $x_{i, j}$

$$
\left|x_{i, j}-\frac{q^{n}-1}{n q(q-1)}\right| \leq q^{\frac{n-2}{2}}+\frac{q^{\frac{n}{2}}-1}{q(q-1)}+\frac{n}{2} q^{\frac{n-4}{4}}<\frac{2}{q-1} q^{\frac{n}{2}} .
$$

Suppose that the constant $c=a_{j} \in \mathbb{F}_{q}^{\times}$, where $1 \leq j \leq q-1$, is such that $\rho=\operatorname{ord}(c)$. Let $x_{r, j}=\max \left\{x_{i, j}: i \in R_{j}\right\}$. Then we have the following result.

Lemma 28. If $c=a_{j}$ is a given constant from $\mathbb{F}_{q}^{\times}$, for some $1 \leq j \leq q-1$, then

$$
\frac{N(n, j, q)}{q-1}-\frac{q^{n-1}-1}{n(q-1)^{2}}-\frac{2 q^{\frac{n}{2}}}{(q-1)^{2}} \leq x_{r, j} \leq \frac{N(n, j, q)}{A_{r, j}}-\frac{q^{n-1}-1}{n(q-1) A_{r, j}}+\frac{2 q^{\frac{n}{2}}}{(q-1) A_{r, j}} .
$$

Proof. From Equation (4.6) we have

$$
\frac{q^{n-1}-1}{n(q-1)}-\frac{2 q^{\frac{n}{2}}}{q-1} \leq y_{0, j} \leq \frac{q^{n-1}-1}{n(q-1)}+\frac{2 q^{\frac{n}{2}}}{q-1} .
$$


By adding $\sum_{i \in R_{j}} A_{i, j} x_{i, j}$ to each expression in this inequality, we have

$$
\frac{q^{n-1}-1}{n(q-1)}-\frac{2 q^{\frac{n}{2}}}{q-1}+\sum_{i \in R_{j}} A_{i, j} x_{i, j} \leq N(n, j, q) \leq \frac{q^{n-1}-1}{n(q-1)}+\frac{2 q^{\frac{n}{2}}}{q-1}+\sum_{i \in R_{j}} A_{i, j} x_{i, j}
$$

Then applying the lower and upper bounds for $\sum_{i \in R_{j}} A_{i, j} x_{i, j}$, we have

$$
\frac{q^{n-1}-1}{n(q-1)}-\frac{2 q^{\frac{n}{2}}}{q-1}+A_{r, j} x_{r, j} \leq N(n, j, q) \leq \frac{q^{n-1}-1}{n(q-1)}+\frac{2 q^{\frac{n}{2}}}{q-1}+(q-1) x_{r, j}
$$

which implies the result.

Next we provide lower and upper bounds for $x_{r, j}$ in terms of $n$ and $q-1$, instead of $N(n, j, q)$. We need to find lower and upper bounds for $N(n, j, q)$.

Definition 29. Let $q$ and $n$ be two positive integers, and the prime factorization of $q^{n}-1$ be given by $q^{n}-1=p_{1}^{e_{1}} p_{2}^{e_{2}} \ldots p_{t}^{e_{t}}$, where $p_{t}$ is the largest prime factor of $q^{n}-1$. Then, the pair $(q, n)$ is said to be a lps (largest prime survives) pair of integers, if $p_{t} \nmid q^{m}-1$, for $m<n$.

Experimental data show that for any $q$, there exist many $n$ 's such that $(q, n)$ is a lps pair. We also found some sporadic pairs $(q, n)$ that are not lps pairs. Let $v=p_{t}^{e_{t}}$, and $m_{r} \mid q-1$ be fixed, where $1 \leq m_{r} \leq q-1$. We let $m_{r}=\rho=\operatorname{ord}(c)$. We use this notation for the rest of this chapter. Then suppose that $D_{\rho, v}$ is the subset of $D_{n}$ defined by those $r$ which $v$ divides them, that is

$$
D_{\rho, v}=\left\{r \in D_{n}: r=m_{r} d_{r}, m_{r}=\rho, v \mid r\right\}
$$

Lemma 30. Let $(q, n)$ be a lps pair of integers. Suppose that $p_{t}$ is the largest prime in the prime factorization of $q^{n}-1$, and $m_{r} \mid q-1$ be fixed as $\rho=\operatorname{ord}(c)$, where we 
are using our previous notation for $r$ and $m_{r}$. Then for all $r \in D_{\rho, v}$ we have

$$
\frac{1}{n \phi(\rho)} \sum_{r \in D_{\rho, v}} \phi(r)=\left(1-\frac{1}{p_{t}}\right) \frac{q^{n}-1}{n(q-1)}
$$

Proof. Let $q-1=p_{1}^{g_{1}} \ldots p_{k}{ }^{g_{k}}$ be the prime factorization of $q-1$, where $g_{i} \geq 1$, for $i \in S_{1}=\{1, \ldots, k\}$. Assume that $m_{r}=\rho$ is a fixed divisor of $q-1$. Then $m_{r}=p_{1}{ }^{l_{1}} \ldots p_{k}{ }^{l_{k}}$, where $0 \leq l_{i} \leq g_{i}$, for $i \in S_{1}$. Each $r \in D_{\rho, v}$ is $r=m_{r} d_{r}$, where $m_{r}=\rho$, and $v=p_{t}^{e_{t}} \mid r$. By Lemma 26 such $r$ can be given by $r=\frac{q^{n}-1}{R}$, where

$$
\operatorname{gcd}(R, q-1)=\frac{q-1}{m_{r}}={p_{1}}^{g_{1}-l_{1}} \ldots p_{k}^{g_{k}-l_{k}}
$$

Since $v \mid r$ and $v \mid q^{n}-1$ thus $v \nmid R$. Then $R \mid q^{n}-1$ implies that $R$ can be given as

$$
R={p_{1}}^{g_{1}-l_{1}} \ldots p_{k}^{g_{k}-l_{k}} p_{k+1}^{c_{k+1}} \ldots p_{t-1}^{c_{t-1}}
$$

where $0 \leq c_{i} \leq e_{i}$, for all $i \in S_{2}-\{t\}=\{k+1, \ldots, t-1\}$. Now, each $r \in D_{\rho, v}$ can be considered as $r=p_{1}^{e_{1}-g_{1}+l_{1}} \ldots p_{k}^{e_{k}-g_{k}+l_{k}} p_{k+1} d_{k+1} \ldots p_{t-1} d_{t-1} p_{t}^{e_{t}}$, such that $d_{i}=e_{i}-c_{i}$, for $i \in S_{2}-\{t\}$. We have

$$
\begin{aligned}
\frac{1}{n \phi(\rho)} \sum_{r \in D_{\rho, v}} \phi(r) & =\frac{1}{n \phi\left(p_{1}^{i_{1}} \ldots p_{k}^{\left.l_{k}\right)}\right.} \sum_{d_{k+1}, \ldots, d_{t-1}} \phi\left(p_{t}^{e_{t}} \prod_{s=1}^{k} p_{s}^{e_{s}-g_{s}+l_{s}} \prod_{u=k+1}^{t-1} p_{u}^{d_{u}}\right) \\
& =\frac{\phi\left(\prod_{s=1}^{k} p_{s}^{e_{s}-g_{s}+l_{s}}\right)}{n \phi\left(p_{1}{ }^{l_{1}} \ldots p_{k}{ }^{l_{k}}\right)}\left(\prod_{u=k+1}^{t-1} \sum_{d_{u}=0}^{e_{u}} \phi\left(p_{u}{ }^{d_{u}}\right)\right) \phi\left(p_{t}^{e_{t}}\right) \\
& =\frac{\prod_{s=1}^{k}\left(p_{s}-1\right) p_{s}^{e_{s}-g_{s}+l_{s}-1}}{n \prod_{s=1}^{k}\left(p_{s}-1\right) p_{s}^{l_{s}-1}}\left(\prod_{u=k+1}^{t-1} p_{u}{ }^{e_{u}}\right) p_{t}^{e_{t}}\left(1-\frac{1}{p_{t}}\right) \\
& =\frac{\left(1-\frac{1}{p_{t}}\right)}{n}\left(\prod_{s=1}^{t} p_{s}^{e_{s}}\right)\left(\prod_{u=1}^{k} p_{u}{ }^{-g_{u}}\right)=\left(1-\frac{1}{p_{t}}\right) \frac{q^{n}-1}{n(q-1)} .
\end{aligned}
$$


We state now our main result about the bounds for $x_{r, j}$.

Theorem 31. Suppose that $(q, n)$ is a lps pair of integers, and $c=a_{j} \in \mathbb{F}_{q}^{\times}$be such that $\rho=\operatorname{ord}(c)$, for some $1 \leq j \leq q-1$. If $p_{t}$ is the largest prime in the factorization of $q^{n}-1$, then

$$
\begin{gathered}
\frac{\left(1-\frac{1}{p_{t}}\right)\left(q^{n}-1\right)-q^{n-1}-2 n q^{\frac{n}{2}}+1}{n(q-1)^{2}} \\
\leq x_{r, j} \leq \frac{1}{A_{r, j}}\left(\frac{q^{n}-1}{n \rho}-\frac{q^{n-1}-1}{n(q-1)}+\frac{2 q^{\frac{n}{2}}}{q-1}\right) .
\end{gathered}
$$

Proof. For a given $m_{r}=\rho$, using the definition of $D_{\rho, v}$ and that $(q, n)$ is a lps pair, we have

$$
N(n, j, q)=\frac{1}{n \phi(\rho)} \sum_{\substack{r \in D_{n} \\ m_{r}=\rho}} \phi(r) \geq \frac{1}{n \phi(\rho)} \sum_{r \in D_{\rho, v}} \phi(r) .
$$

Therefore, using Lemma 30, a lower bound for $N(n, j, q)$ can be given as

$$
N(n, j, q) \geq\left(1-\frac{1}{p_{t}}\right) \frac{q^{n}-1}{n(q-1)}
$$

Using Lemma 28, we obtain the stated lower bound for $x_{r, j}$. An upper bound for $N(n, j, q)$ can be derived using

$$
N(n, j, q)=\frac{1}{n \phi(\rho)} \sum_{\substack{r \in D_{n} \\ m_{r}=\rho}} \phi(r) \leq \frac{1}{n \phi(\rho)} \sum_{\substack{r q^{n}=1 \\ m_{r}=\rho}} \phi(r)
$$


where the sum at the right-hand side is simply

$$
\frac{1}{n \phi(\rho)} \sum_{\rho d_{r} \mid q^{n}-1} \phi\left(\rho d_{r}\right)=\frac{1}{n} \sum_{d_{r} \mid \frac{q^{n}-1}{\rho}} \phi\left(d_{r}\right)=\frac{q^{n}-1}{n \rho}
$$

Using Lemma 28, this implies the stated upper bound for $x_{r, j}$.

The following tables compare our lower bound with other lower bounds for $x_{r, j}$. We choose different $n$ and $q$ such that $(q, n)$ is a lps pair of integers, and they are small enough to compute the numbers in the tables. Table 4.2 gives Wan and Moisio's bounds for $x_{r, j}$. For each entry $(a, b)$ of Table 4.2 , the number $a$ represents the lower bound obtained by Wan and $b$ is Moisio's lower bound. Table 4.3 gives our lower bound for $x_{r, j}$ for the same finite fields and degrees.

Table 4.2: Wan and Moisio's bounds for $x_{r, j}$.

\begin{tabular}{|c|c|c|c|}
\hline \multirow{2}{*}{$\mathbb{F}_{q}$} & \multicolumn{3}{|c|}{ Degree $n$} \\
\cline { 2 - 4 } & 4 & 7 & 11 \\
\hline $\mathbb{F}_{4}$ & $(0,0)$ & $(140.19,142.55)$ & $(31216.48,31030.21)$ \\
\hline $\mathbb{F}_{5}$ & $(0,0)$ & $(438.2,476.5)$ & $(220040.28,220107.19)$ \\
\hline $\mathbb{F}_{7}$ & $(0,4.14)$ & $(2412.24,2634.88)$ & $(4267800.61,4272351.16)$ \\
\hline $\mathbb{F}_{8}$ & $(0,7.16)$ & $(4729.24,5126.36)$ & $(13919422.13,13931249.46)$ \\
\hline $\mathbb{F}_{9}$ & $(0,10.66)$ & $(8552.73,9198.47)$ & $(39574237.19,39600149.44)$ \\
\hline $\mathbb{F}_{11}$ & $(0,19.18)$ & $(23416.12,24845.44)$ & $(235649092.99,235740989.11)$ \\
\hline $\mathbb{F}_{13}$ & $(0,29.7)$ & $(54067.13,56777.94)$ & $(1044017409.66,1044270464.84)$ \\
\hline
\end{tabular}

Table 4.3: Our bound for $x_{r, j}$

\begin{tabular}{|c|c|c|c|}
\hline \multirow{2}{*}{$\mathbb{F}_{q}$} & \multicolumn{3}{|c|}{ Degree $n$} \\
\cline { 2 - 4 } & 4 & 7 & 11 \\
\hline $\mathbb{F}_{4}$ & 1.74 & 164.56 & 31257.89 \\
\hline $\mathbb{F}_{5}$ & 3.94 & 523.06 & 221072.5 \\
\hline $\mathbb{F}_{7}$ & 8.24 & 2750.06 & 4277440.6 \\
\hline $\mathbb{F}_{8}$ & 14.07 & 5272.63 & 13940889.49 \\
\hline $\mathbb{F}_{9}$ & 19.62 & 9411.91 & 39605439.16 \\
\hline $\mathbb{F}_{11}$ & 30.25 & 25219.11 & 235783942.58 \\
\hline $\mathbb{F}_{13}$ & 40.51 & 57351.98 & 1044301207.22 \\
\hline
\end{tabular}


To compare our lower bound and Moisio's lower bound in general, we look at their difference,

$$
\frac{\left(1-\frac{1}{p_{t}}\right)\left(q^{n}-1\right)-q^{n-1}-2 n q^{\frac{n}{2}}+1}{n(q-1)^{2}}-\frac{q^{n}-1}{n q(q-1)}+\frac{2}{q-1} q^{\frac{n}{2}}
$$

or,

$$
-\frac{\left(q^{n}-1\right)}{n(q-1)^{2} p_{t}}+\frac{2(q-2)}{(q-1)^{2}} q^{\frac{n}{2}}+\frac{1}{n q(q-1)} .
$$

Therefore, if the number $p_{t}$ is of size $q^{\frac{n}{2}-1}$ or larger, then this difference is positive, and so our bound is better. Checking different $q$ and $n$, this situation happens very often.

Remark. If $A_{r, j}=\rho=q-1$, our upper bound is better than Moisio's upper bound. In the next section we show that this is the case if $n$ is a multiple of $q-1$. Examples are given in the next section.

\subsection{The Special Case $n$ Being a Multiple of $q-1$}

Suppose that the degree of the polynomials is fixed as $n=a(q-1)$, for some positive integer $a$. Then we have the following results.

Lemma 32. Let $1 \leq m \leq n-1, q-1 \nmid m$, and $n=a(q-1)$, for some positive integer $a$. Then $(q-1)^{2} \mid q^{n}-1$ and $(q-1)^{2} \nmid q^{m}-1$. In particular, $n^{2} \mid a^{2}\left(q^{n}-1\right)$, and $n^{2} \nmid a^{2}\left(q^{m}-1\right)$.

Proof. For any integer $0 \leq i \leq m-1$ we have $q^{i} \equiv 1(\bmod q-1)$. If we add these $m$ equations, then

$$
q^{m-1}+q^{m-2}+\cdots+q+1 \equiv m \not \equiv 0 \quad(\bmod q-1) .
$$


Therefore $q-1 \nmid q^{m-1}+q^{m-2}+\cdots+q+1$, and if we multiply it by $q-1$, then $(q-1)^{2} \nmid q^{m}-1$. Also for any $0 \leq i \leq n-1$ we have $q^{i} \equiv 1(\bmod q-1)$, and then

$$
q^{n-1}+q^{n-2}+\cdots+q+1 \equiv n \equiv 0 \quad(\bmod q-1)
$$

Hence, multiplying by $q-1$, we have the $(q-1)^{2} \mid q^{n}-1$. Similarly, for $n=a(q-1)$ we have $n^{2} \mid a^{2}\left(q^{n}-1\right)$, and $n^{2} \nmid a^{2}\left(q^{m}-1\right)$.

Lemma 33. Suppose that $n=a(q-1)$, for some integer a. Let $r=\frac{q^{n}-1}{R}$ such that $R \mid q^{n}-1$, and $\operatorname{gcd}(R, q-1)=1$, that is $m_{r}=q-1$. Then $r \nmid\left(q^{m}-1\right)$, for all $m=1,2, \ldots, n-1$, and $m$ is not a multiple of $q-1$.

Proof. Let the prime factorization of $q-1$ and $q^{n}-1$ be given as $q-1=p_{1}^{g_{1}} \ldots p_{k}^{g_{k}}$ and

$$
q^{n}-1=p_{1}^{e_{1}} \ldots p_{k}^{e_{k}} p_{k+1}^{e_{k+1}} \ldots p_{t}^{e_{t}}
$$

If $n=a(q-1)$, then by Lemma 32 we have $(q-1)^{2} \mid q^{n}-1$. Therefore in the prime factorization of $q^{n}-1$ we have $e_{i} \geq 2 g_{i}$, for $i=1, \ldots, k$. Since $\operatorname{gcd}(R, q-1)=1$, then the prime factors of $R$ are from the set $\left\{p_{k+1}, \ldots, p_{t}\right\}$. Thus $r$ can be considered as

$$
r=\frac{\left(q^{n}-1\right)}{R}=p_{1}^{e_{1}} \ldots p_{k}^{e_{k}} p_{k+1}^{f_{k+1}} \ldots p_{t}^{f_{t}}
$$

where $0 \leq f_{i} \leq e_{i}$, for $i \in S_{2}$. It is clear that $(q-1)^{2} \mid r$. Now we show that $r \nmid q^{m}-1$, for $m=1,2, \ldots, n-1$ and $q-1 \nmid m$. Suppose $r \mid q^{m}-1$. Then $a^{2} r \mid a^{2}\left(q^{m}-1\right)$. Since $(q-1)^{2} \mid r$, we have $n^{2}=a^{2}(q-1)^{2} \mid a^{2} r$, and therefore $n^{2} \mid a^{2}\left(q^{m}-1\right)$. This contradicts Lemma 32 . To prove $q-1 \nmid m$ we use a similar contradiction.

Let $c \in \mathbb{F}_{q}^{\times}$be such that $\rho=\operatorname{ord}(c)$. The constant $c$ can be a primitive, or non 
primitive constant. For different $r$, in the relation

$$
N(n, c, q)=\frac{1}{n \phi(\rho)} \sum_{\substack{r \in D_{n} \\ m_{r}=\rho}} \phi(r)
$$

the value of $m_{r}$ is fixed as $m_{r}=\rho$. Let $c \in \mathbb{F}_{q}^{\times}$represent any primitive element. Then obviously $\rho=q-1$, and let $r \in D_{n}$ be such that $m_{r}=\rho=q-1$.

Theorem 34. Let $n=a(q-1)$, for some integer $a$, and $c \in \mathbb{F}_{q}^{\times}$be primitive. Then

$$
N(n, c, q) \leq \frac{q^{n}-1}{a(q-1)^{2}}
$$

In addition, if $q$ and $n$ are such that $p_{1}^{e_{1}} p_{2}^{e_{2}} \ldots p_{k}^{e_{k}} \nmid q^{m}-1$, for $m$ multiple of $q-1$ and $m<n$, where $q^{n}-1=p_{1}^{e_{1}} p_{2}^{e_{2}} \ldots p_{k}^{e_{k}} p_{k+1}^{e_{k+1}} \ldots p_{t}^{e_{t}}$, then $N(n, c, q)=\frac{q^{n}-1}{a(q-1)^{2}}$.

Proof. Let $q-1=p_{1}^{g_{1}} p_{2}^{g_{2}} \ldots p_{k}^{g_{k}}$ be the prime factorization of $q-1$, where $g_{i} \geq 1$, for $i \in S_{1}$. Similarly, $q^{n}-1=p_{1}^{e_{1}} p_{2}^{e_{2}} \ldots p_{k}^{e_{k}} p_{k+1}^{e_{k+1}} \ldots p_{t}^{e_{t}}$, such that $e_{i} \geq g_{i} \geq 1$, when $i \in S_{1}$, and $e_{i} \geq 1$, when $i \in S_{2}$. Since $c \in \mathbb{F}_{q}^{\times}$is primitive, so $\rho=q-1$. Let $n=a(q-1)$, then by Equation (4.8) we have

$$
N(n, c, q)=\frac{1}{a(q-1) \phi(q-1)} \sum_{\substack{r \in D_{n} \\ m_{r}=q-1}} \phi(r)
$$

For any $r=\frac{q^{n}-1}{R}$, where $\operatorname{gcd}(R, q-1)=\frac{q-1}{m_{r}}=1$ and $R=p_{k+1}{ }^{c_{k+1}} \ldots p_{t}^{c_{t}}$ with $0 \leq c_{i} \leq e_{i}$ for $i \in S_{2}$, we can write

$$
r=\frac{q^{n}-1}{R}=p_{1}^{e_{1}} \ldots p_{k}^{e_{k}} p_{k+1}^{f_{k+1}} \ldots p_{t}^{f_{t}}
$$

where $f_{i}=e_{i}-c_{i}$, for $i \in S_{2}$. By Lemma 33, $r \nmid q^{m}-1$, for all $m$ not multiple of $q-1$, and $m<n$. Since $p_{1}^{e_{1}} p_{2}^{e_{2}} \ldots p_{k}^{e_{k}} \nmid q^{m}-1$ for all $m$ multiple of $q-1$ and $m<n$, 
we conclude that any $r$ of this form is in $D_{n}$. Hence, the number $N(n, c, q)$ can be given by

$$
\begin{aligned}
N(n, c, q) & =\frac{1}{a\left(p_{1}^{g_{1}} \ldots p_{k}^{g_{k}}\right) \phi\left(p_{1}^{g_{1}} \ldots p_{k}^{g_{k}}\right)} \sum_{f_{k+1}, \ldots, f_{t}} \phi\left(p_{1}^{e_{1}} \ldots p_{k}^{e_{k}} p_{k+1}^{f_{k+1}} \ldots p_{t}^{f_{t}}\right) \\
& =\frac{\phi\left(p_{1}^{e_{1}} \ldots p_{k}^{e_{k}}\right)}{a\left(p_{1}^{g_{1}} \ldots p_{k}^{g_{k}}\right) \phi\left(p_{1}^{g_{1}} \ldots p_{k}^{g_{k}}\right)} \sum_{f_{k+1}, \ldots, f_{t}} \phi\left(p_{k+1}^{f_{k+1}} \ldots p_{t}^{f_{t}}\right) \\
& =\frac{\left(p_{1}^{e_{1}-g_{1}} \ldots p_{k}^{e_{k}-g_{k}}\right)}{a\left(p_{1}^{g_{1}} \ldots p_{k}^{g_{k}}\right)} \prod_{s=k+1}^{t} \sum_{f_{s}=0}^{e_{s}} \phi\left(p_{s}^{f_{s}}\right) \\
& =\frac{p_{1}^{e_{1}} \ldots p_{k}^{e_{k}}}{a\left(p_{1}^{2 g_{1}} \ldots p_{k}^{2 g_{k}}\right)} \prod_{s=k+1}^{t} p_{s}^{e_{s}}=\frac{q^{n}-1}{a(q-1)^{2}} .
\end{aligned}
$$

Finally, we observe that if $p_{1}^{e_{1}} p_{2}^{e_{2}} \ldots p_{k}^{e_{k}} \mid q^{m}-1$, for some $m$ multiple of $q-1$ and $m<n$, then we can only conclude that $N(n, c, q)<\frac{q^{n}-1}{a(q-1)^{2}}$.

Suppose that $c^{\prime} \in \mathbb{F}_{q}^{\times}$is any non primitive constant, which is related to $r^{\prime} \in D_{n}$, where $r^{\prime}=m_{r^{\prime}} d_{r^{\prime}}$ and $m_{r^{\prime}}=\rho^{\prime}=\operatorname{ord}\left(c^{\prime}\right)<q-1$. Clearly $r^{\prime} \in D_{n}$ can be considered as $r^{\prime}=\frac{q^{n}-1}{R^{\prime}}$, with $\operatorname{gcd}\left(q-1, R^{\prime}\right)=\frac{q-1}{m_{r^{\prime}}}>1$. Moreover $r^{\prime} \nmid q^{m}-1$, for $1 \leq m \leq n-1$. Let us remove the last condition and define $\widehat{r}^{\prime}=\frac{q^{n}-1}{\widehat{R}^{\prime}}$, such that $\widehat{R}^{\prime} \mid q^{n}-1$, and $\operatorname{gcd}\left(q-1, \widehat{R}^{\prime}\right)=\frac{q-1}{m_{r^{\prime}}}>1$.

Lemma 35. Let $c^{\prime} \in \mathbb{F}_{q}^{\times}$be non primitive, where $\rho^{\prime}=\operatorname{ord}\left(c^{\prime}\right)=m_{r^{\prime}}<q-1$. Then

$$
\frac{1}{n \phi\left(\rho^{\prime}\right)} \sum_{\widehat{r}^{\prime}} \phi\left(\widehat{r}^{\prime}\right)=\frac{q^{n}-1}{a(q-1)^{2}}
$$

where the sum runs over all $\widehat{r}^{\prime}$, defined as $\widehat{r}=\frac{q^{n}-1}{\widehat{R}^{\prime}}$, with $\operatorname{gcd}\left(q-1, \widehat{R}^{\prime}\right)=\frac{q-1}{m_{r^{\prime}}}=\frac{q-1}{\rho^{\prime}}$.

Proof. Suppose $c^{\prime} \in \mathbb{F}_{q}^{\times}$be such that $\rho^{\prime}=\operatorname{ord}\left(c^{\prime}\right)=m_{r^{\prime}}=p_{1}^{l_{1}} \ldots p_{k}^{l_{k}} \mid q-1$, where 
$0 \leq l_{i} \leq g_{i}$, for $i \in S_{1}$. Let $\widehat{r}=\frac{q^{n}-1}{\hat{R}^{\prime}}$, where

$$
\operatorname{gcd}\left(q-1, \widehat{R}^{\prime}\right)=\frac{q-1}{m_{r^{\prime}}}=p_{1}^{g_{1}-l_{1}} \ldots p_{k}^{g_{k}-l_{k}}
$$

This implies that $\widehat{R}^{\prime}=p_{1}^{g_{1}-l_{1}} \ldots p_{k}^{g_{k}-l_{k}} p_{k+1}{ }^{c_{k+1}} \ldots p_{t}^{c_{t}}$, with $0 \leq c_{i} \leq e_{i}$, for $i \in S_{2}$. Therefore $\widehat{r}$ can be considered as

$$
\begin{aligned}
\widehat{r}^{\prime} & =\frac{q^{n}-1}{\widehat{R}^{\prime}}=\frac{p_{1}^{e_{1}} p_{2}^{e_{2}} \ldots p_{k}^{e_{k}} p_{k+1}^{e_{k+1}} \ldots p_{t}^{e_{t}}}{p_{1}^{g_{1}-l_{1}} \ldots p_{k}^{g_{k}-l_{k}} p_{k+1}{ }^{c_{k+1}} \ldots p_{t}^{c_{t}}} \\
& =p_{1}^{e_{1}-g_{1}+l_{1}} \ldots p_{k}^{e_{k}-g_{k}+l_{k}} p_{k+1}^{e_{k+1}-c_{k+1}} \ldots p_{t}^{e_{t}-c_{t}} \\
& =p_{1}^{e_{1}-g_{1}+l_{1}} \ldots p_{k}^{e_{k}-g_{k}+l_{k}} p_{k+1}^{d_{k}+1} \ldots p_{t}^{d_{t}},
\end{aligned}
$$

such that $d_{i}=e_{i}-c_{i}$, for $i \in S_{2}$. Then

$$
\begin{aligned}
\frac{1}{n \phi\left(\rho^{\prime}\right)} \sum_{\widehat{r}^{\prime}} \phi\left(\widehat{r}^{\prime}\right) & =\frac{1}{a\left(p_{1}^{g_{1}} \ldots p_{k}^{g_{k}}\right) \phi\left(p_{1}^{l_{1}} \ldots p_{k}^{l_{k}}\right)} \sum_{d_{k+1}, \ldots, d_{t}} \phi\left(\prod_{s=1}^{k} p_{s}{ }^{e_{s}-g_{s}+l_{s}} \cdot \prod_{u=k+1}^{t} p_{u}{ }^{d_{u}}\right) \\
& =\frac{\phi\left(\prod_{s=1}^{k} p_{s}{ }^{e_{s}-g_{s}+l_{s}}\right)}{a\left(p_{1}^{g_{1}} \ldots p_{k}^{g_{k}}\right) \phi\left(p_{1}^{l_{1}} \ldots p_{k}{ }^{l_{k}}\right)}\left(\prod_{u=k+1}^{t} \sum_{d_{u}=0}^{e_{u}} \phi\left(p_{u}{ }^{{ }^{\prime}}\right)\right) \\
& =\frac{\prod_{s=1}^{k}\left(p_{s}-1\right) p_{s}{ }^{e_{s}-g_{s}+l_{s}-1}}{a \prod_{s=1}^{k}\left(p_{s}-1\right) p_{s}{ }_{g_{s}+l_{s}-1}^{t}}\left(\prod_{u=k+1}^{t} p_{u}^{e_{u}}\right) \\
& =\left(\frac{1}{a} \prod_{s=1}^{k} p_{s}^{e_{s}-2 g_{s}}\right)\left(\prod_{u=k+1}^{t} p_{u}^{e_{u}}\right)=\frac{q^{n}-1}{a(q-1)^{2}} .
\end{aligned}
$$

Theorem 36. If $n=a(q-1)$, for some integer $a$. Then for any non primitive constant $c^{\prime} \in \mathbb{F}_{q}^{\times}$, we have $N\left(n, c^{\prime}, q\right) \leq \frac{q^{n}-1}{a(q-1)^{2}}$. 
Proof. For the non primitive $c^{\prime} \in \mathbb{F}_{q}^{\times}$, let $\rho^{\prime}=\operatorname{ord}\left(c^{\prime}\right)$. Then by Lemma 35,

$$
\frac{q^{n}-1}{a(q-1)^{2}}=\frac{1}{n \phi\left(\rho^{\prime}\right)} \sum_{\widehat{r}^{\prime}} \phi\left(\widehat{r}^{\prime}\right) \geq \frac{1}{n \phi\left(\rho^{\prime}\right)} \sum_{\substack{r^{\prime} \in D_{n} \\ m_{r^{\prime}}=\rho^{\prime}}} \phi\left(r^{\prime}\right)=N\left(n, c^{\prime}, q\right),
$$

which proves the statement.

This proves that when $n=a(q-1)$ the maximum value of $N(n, c, q)$ occurs for primitive $c \in \mathbb{F}_{q}^{\times}$. If $n=a(q-1)$, then we have the following restatement of Lemma 27.

Lemma 37. Let $n=a(q-1)$ for some integer $a$, and $c \in \mathbb{F}_{q}^{\times}$be any constant. Then for any two nonzero traces $\gamma$ and $\delta$, we have $N_{\gamma}(n, c, q)=N_{\delta}(n, c, q)$.

Proof. Let $\gamma$ and $\delta$ be two nonzero elements in $\mathbb{F}_{q}$, and $n=a(q-1)$. Therefore

$$
\left(\frac{\delta}{\gamma}\right)^{n}=\left(\frac{\delta}{\gamma}\right)^{a(q-1)}=\left(\left(\frac{\delta}{\gamma}\right)^{q-1}\right)^{a}=1^{a}=1 .
$$

Then from Lemma 27 we have

$$
N_{\gamma}(n, c, q)=N_{\delta}\left(n, c\left(\frac{\delta}{\gamma}\right)^{n}, q\right)=N_{\delta}(n, c, q)
$$

This means that, when $n=a(q-1)$, for any $i, l \in\{1,2, \ldots, q-1\}$, and $j \in$ $\{0,1, \ldots, q-1\}$, we have $x_{i, j}=x_{l, j}$. So we let $x_{j}$ represent $x_{i, j}$. Moreover, for $\gamma \in \mathbb{F}_{q}^{\times}$, let $N_{\gamma}\left(n, a_{j}, q\right)=x_{j}$, and $N_{0}\left(n, a_{j}, q\right)=y_{j}$, where $j \in\{1,2, \ldots, q-1\}$; see Table 4.4 . In Table 4.4, we have the same rows for different $\gamma \in \mathbb{F}_{q}^{\times}$. In this case, let $A_{j}$ be the number of repeated entries of the column $a_{j}$, where $1 \leq j \leq q-1$. Clearly $A_{j}=q-1$. 
Thus for a given nonzero constant $c$ (or $c^{\prime}$ ), Equation (4.4) changes to

$$
y_{c}+(q-1) x_{c}=N(n, c, q)
$$

Table 4.4: Distribution of polynomials of degree $n=a(q-1)$ over a finite field $\mathbb{F}_{q}$.

\begin{tabular}{|c|c|c|c|c|c|c|}
\hline $\operatorname{Tr}$ Cons & $a_{1}$ & $\cdots$ & $a_{j}$ & $\cdots$ & $a_{q-1}$ & Total \\
\hline$a_{0}$ & $y_{1}$ & $\cdots$ & $y_{j}$ & $\cdots$ & $y_{q-1}$ & $N_{0}(n, q)$ \\
\hline$a_{1}$ & $x_{1}$ & $\cdots$ & $x_{j}$ & $\cdots$ & $x_{q-1}$ & $N_{1}(n, q)$ \\
\hline$\vdots$ & $\vdots$ & & $\vdots$ & & $\vdots$ & $\vdots$ \\
\hline$a_{q-1}$ & $x_{1}$ & $\cdots$ & $x_{j}$ & $\cdots$ & $x_{q-1}$ & $N_{q-1}(n, q)$ \\
\hline Total & $N(n, 1, q)$ & $\cdots$ & $N(n, j, q)$ & $\cdots$ & $N(n, q-1, q)$ & $N(n, q)$ \\
\hline
\end{tabular}

Using Equation (4.9) and Theorem 34, we have the following bounds for $x_{c}$.

Theorem 38. Let $n=a(q-1)$, such that $q-1=p_{1}^{g_{1}} p_{2}^{g_{2}} \ldots p_{k}^{g_{k}}$ and

$$
q^{n}-1=p_{1}^{e_{1}} p_{2}^{e_{2}} \ldots p_{k}^{e_{k}} p_{k+1}^{e_{k+1}} \ldots p_{t}^{e_{t}}
$$

which satisfy $p_{1}^{e_{1}} p_{2}^{e_{2}} \ldots p_{k}^{e_{k}} \nmid q^{m}-1$, for all $m$ multiple of $q-1$ and $m<n$. Then for any primitive constant $c \in \mathbb{F}_{q}^{\times}$we have

$$
\left|x_{c}-\frac{q^{n}-q^{n-1}}{a(q-1)^{3}}\right| \leq \frac{2}{(q-1)^{2}} q^{\frac{n}{2}} .
$$

Proof. Let $n=a(q-1)$, for some integer $a$, and $c=a_{j}$ be a primitive constant from $\mathbb{F}_{q}^{\times}$, for some $1 \leq j \leq q-1$. Then $A_{j}=q-1$, and $\rho=\operatorname{ord}(c)=q-1$. By Lemma 28 the lower and upper bounds for $x_{c}$ can be given by

$$
\frac{N(n, j, q)}{q-1}-\frac{q^{n-1}-1}{n(q-1)^{2}}-\frac{2 q^{\frac{n}{2}}}{(q-1)^{2}} \leq x_{c} \leq \frac{N(n, j, q)}{A_{j}}-\frac{q^{n-1}-1}{n(q-1) A_{j}}+\frac{2 q^{\frac{n}{2}}}{(q-1) A_{j}}
$$


or

$$
\frac{N(n, j, q)}{q-1}-\frac{q^{n-1}-1}{a(q-1)^{3}}-\frac{2 q^{\frac{n}{2}}}{(q-1)^{2}} \leq x_{c} \leq \frac{N(n, j, q)}{q-1}-\frac{q^{n-1}-1}{a(q-1)^{3}}+\frac{2 q^{\frac{n}{2}}}{(q-1)^{2}}
$$

Suppose that $q$ and $n$ are such that $p_{1}^{e_{1}} p_{2}^{e_{2}} \ldots p_{k}^{e_{k}} \nmid q^{m}-1$, for $m$ multiple of $q-1$, and $m<n$. Then by Theorem 34 we have $N(n, j, q)=\frac{q^{n}-1}{a(q-1)^{2}}$, and the bounds for $x_{c}$ change to

$$
\frac{q^{n}-1}{a(q-1)^{3}}-\frac{q^{n-1}-1}{a(q-1)^{3}}-\frac{2 q^{\frac{n}{2}}}{(q-1)^{2}} \leq x_{c} \leq \frac{q^{n}-1}{a(q-1)^{3}}-\frac{q^{n-1}-1}{a(q-1)^{3}}+\frac{2 q^{\frac{n}{2}}}{(q-1)^{2}}
$$

or

$$
\frac{q^{n}-q^{n-1}}{a(q-1)^{3}}-\frac{2 q^{\frac{n}{2}}}{(q-1)^{2}} \leq x_{c} \leq \frac{q^{n}-q^{n-1}}{a(q-1)^{3}}+\frac{2 q^{\frac{n}{2}}}{(q-1)^{2}}
$$

which means

$$
\left|x_{c}-\frac{q^{n}-q^{n-1}}{a(q-1)^{3}}\right| \leq \frac{2}{(q-1)^{2}} q^{\frac{n}{2}}
$$

We note that the upper bound does not require the condition $p_{1}^{e_{1}} p_{2}^{e_{2}} \ldots p_{k}^{e_{k}} \nmid q^{m}-1$, for $m$ multiple of $q-1$ and $m<n$.

The difference between our lower bound and Moisio's lower bound is

$$
\frac{q^{n}-q^{n-1}}{a(q-1)^{3}}-\frac{2}{(q-1)^{2}} q^{\frac{n}{2}}-\frac{q^{n}-1}{a q(q-1)^{2}}+\frac{2}{q-1} q^{\frac{n}{2}}=\frac{1}{(q-1)^{2}}\left(2 q^{\frac{n}{2}}(q-2)+\frac{1}{a q}\right)
$$

which is always positive. This shows that our lower bound is better.

The difference between our upper bound and Moisio's upper bound is

$$
\frac{q^{n}-q^{n-1}}{a(q-1)^{3}}+\frac{2}{(q-1)^{2}} q^{\frac{n}{2}}-\frac{q^{n}-1}{a q(q-1)^{2}}-\frac{2}{q-1} q^{\frac{n}{2}}=\frac{1}{(q-1)^{2}}\left(2 q^{\frac{n}{2}}(2-q)+\frac{1}{a q}\right)
$$

which is always negative if $q \geq 3$. This shows that our upper bound is better. 
Tables 4.5 and 4.6 compare our lower and upper bounds with Wan bounds given in Equation 4.5, and Moisio bounds given in Equation 4.7 for different finite fields $\mathbb{F}_{q}$, and degree $n=q-1$. In each column of these two tables, the entry $[x, y]$ of the table represents the corresponding [lower bound, upper bound]. The last column of table 4.6 gives the minimum and the maximum of $x_{c}$ computed by a Maple code.

Table 4.5: Wan and Moisio's bounds for $x_{c}$, when $n=q-1$.

\begin{tabular}{|c|c|c|}
\hline$q$ & Wan $[22]$ & Moisio $[15]$ \\
\hline 4 & {$[0,9.78]$} & {$[0,5.39]$} \\
\hline 5 & {$[0,26.56]$} & {$[0,16]$} \\
\hline 7 & {$[295.36,638.36]$} & {$[401.78,531.94]$} \\
\hline 8 & {$[4729.24,5970.52]$} & {$[5126.36,5573.38]$} \\
\hline 9 & {$[72273.52,74938.92]$} & {$[73877.78,75590]$} \\
\hline 11 & {$[23531161,23627792]$} & {$[23563189,23595764]$} \\
\hline
\end{tabular}

Table 4.6: Our bounds and [Min, Max] for $x_{c}$, when $n=q-1$.

\begin{tabular}{|c|c|c|}
\hline$q$ & Our Bounds & {$[$ Min, Max $]$} \\
\hline 4 & {$[0,4.407]$} & 1 \\
\hline 5 & {$[3.109,12.484]$} & {$[7,8]$} \\
\hline 7 & {$[438.273,495.439]$} & {$[466,471]$} \\
\hline 8 & {$[5261.212,5438.537]$} & 5344 \\
\hline 9 & {$[74426.342,75041.436]$} & 74691 \\
\hline 11 & {$[23,574,645,23,584,308]$} & {$[23,578,887,23,580,368]$} \\
\hline
\end{tabular}

Now let $c^{\prime} \in \mathbb{F}_{q}^{\times}$be a non primitive constant with $\rho^{\prime}=\operatorname{ord}\left(c^{\prime}\right)$. Using Theorem 31 and Theorem 36, we have the following bounds for $x_{c^{\prime}}$.

Theorem 39. Suppose $(q, n)$ is a lps pair, and $n=a(q-1)$, for some integer a. Let $c^{\prime} \in \mathbb{F}_{q}^{\times}$be a non primitive constant. If $p_{t}$ is the largest prime in the factorization of $q^{n}-1$, then we have

$$
\frac{\left(1-\frac{1}{p_{t}}\right)\left(q^{n}-1\right)-q^{n-1}-2 a(q-1) q^{\frac{n}{2}}+1}{a(q-1)^{3}} \leq x_{c^{\prime}} \leq \frac{q^{n}-q^{n-1}+2 a(q-1) q^{\frac{n}{2}}}{a(q-1)^{3}} .
$$


Proof. Let $n=a(q-1)$, for some integer $a$. For any non primitive constant $c^{\prime} \in \mathbb{F}_{q}^{\times}$ we have $y_{c^{\prime}}+(q-1) x_{c^{\prime}}=N\left(n, c^{\prime}, q\right)$. By Equation (4.6) we have

$$
\frac{q^{n-1}-1}{a(q-1)^{2}}-\frac{2}{q-1} q^{\frac{n}{2}} \leq y_{c^{\prime}} \leq \frac{q^{n-1}-1}{a(q-1)^{2}}+\frac{2}{q-1} q^{\frac{n}{2}} .
$$

If we add $(q-1) x_{c^{\prime}}$ to this inequality, then

$$
\frac{q^{n-1}-1}{a(q-1)^{2}}-\frac{2 q^{\frac{n}{2}}}{q-1}+(q-1) x_{c^{\prime}} \leq N\left(n, c^{\prime}, q\right) \leq \frac{q^{n-1}-1}{a(q-1)^{2}}+\frac{2 q^{\frac{n}{2}}}{q-1}+(q-1) x_{c^{\prime}} .
$$

Therefore, we obtain

$$
\frac{N\left(n, c^{\prime}, q\right)}{q-1}-\frac{q^{n-1}-1}{a(q-1)^{3}}-\frac{2 q^{\frac{n}{2}}}{(q-1)^{2}} \leq x_{c^{\prime}} \leq \frac{N\left(n, c^{\prime}, q\right)}{q-1}-\frac{q^{n-1}-1}{a(q-1)^{3}}+\frac{2 q^{\frac{n}{2}}}{(q-1)^{2}} .
$$

Since $n=a(q-1)$ then by Theorem 36 we have $N\left(n, c^{\prime}, q\right) \leq \frac{q^{n}-1}{a(q-1)^{2}}$, which simplifies the upper bound for $x_{c^{\prime}}$ to

$$
\frac{q^{n}-q^{n-1}+2 a(q-1) q^{\frac{n}{2}}}{a(q-1)^{3}} .
$$

If $(q, n)$ is a lps pair, then with an argument similar to Theorem 31 we have

$$
N\left(n, c^{\prime}, q\right) \geq\left(1-\frac{1}{p_{t}}\right) \frac{q^{n}-1}{n(q-1)}=\left(1-\frac{1}{p_{t}}\right) \frac{q^{n}-1}{a(q-1)^{2}}
$$

Then the lower bound for $x_{c^{\prime}}$ can be given by

$$
\left(1-\frac{1}{p_{t}}\right) \frac{q^{n}-1}{a(q-1)^{3}}-\frac{q^{n-1}-1}{a(q-1)^{3}}-\frac{2 q^{\frac{n}{2}}}{(q-1)^{2}},
$$


or,

$$
\frac{\left(1-\frac{1}{p_{t}}\right)\left(q^{n}-1\right)-q^{n-1}-2 a(q-1) q^{\frac{n}{2}}+1}{a(q-1)^{3}} .
$$

For the same reason as above, our upper bound is better than Moisio's result as long as $q \geq 3$ and our lower bound is better if $p_{t}$ is of size $q^{\frac{n}{2}-1}$ or larger.

Table 4.7 gives Wan and Moisio's bounds for $x_{c^{\prime}}$, when $n=q-1$ and for different finite fields $\mathbb{F}_{q}$. In Table 4.8 our bounds for $x_{c^{\prime}}$ and the interval [Min, Max] computed by a Maple code is given.

Table 4.7: Wan and Moisio's bounds for $x_{c^{\prime}}$, with $n=q-1$.

\begin{tabular}{|r|c|c|}
\hline$q$ & Wan[13] & Moisio[9] \\
\hline 4 & {$[0,9.78]$} & {$[0,5.39]$} \\
\hline 5 & {$[0,26.56]$} & {$[0,16]$} \\
\hline 7 & {$[295.36,638.36]$} & {$[401.78,531.94]$} \\
\hline 8 & {$[4729.24,5970.52]$} & {$[5126.36,5573.38]$} \\
\hline 9 & {$[72273.52,74938.92]$} & {$[73877.78,75590]$} \\
\hline 11 & {$[23,531,161,23,627,792]$} & {$[23,563,189,23,595,764]$} \\
\hline
\end{tabular}

Table 4.8: Our bounds and [Min, Max] for $x_{c^{\prime}}$, with $n=q-1$.

\begin{tabular}{|c|c|c|}
\hline$q$ & Our Bounds & {$[$ Min, Max $]$} \\
\hline 4 & {$[0,3.56]$} & 2 \\
\hline 5 & {$[3.94,10.94]$} & {$[7,8]$} \\
\hline 7 & {$[435.139,485.917]$} & {$[458,471]$} \\
\hline 8 & {$[5272.626,5408.986]$} & {$[5337,5360]$} \\
\hline 9 & {$[74093.32,74938.922]$} & {$[74700,74754]$} \\
\hline 11 & {$[23,574,323,23,582,697]$} & {$[23,578,378,23,579,568]$} \\
\hline
\end{tabular}

Remark. For any given finite field $\mathbb{F}_{q}$ and given degree $n$ such that $q-1 \nmid n$, we know that $A_{r, j}<q-1$. Indeed, let $\gamma$ and $\delta$ be two nonzero elements in $\mathbb{F}_{q}$. Thus, $\left(\frac{\gamma}{\delta}\right)^{n} \neq 1$, and by Lemma 27 we have $N_{\gamma}(n, c, q)=N_{\delta}\left(n, c\left(\frac{\delta}{\gamma}\right)^{n}, q\right) \neq N_{\delta}(n, c, q)$. However, we do not know whether we can still improve upper bounds in this case. 


\section{Chapter 5}

\section{The Number of Irreducible}

\section{Polynomials of Even Degree $n$}

\section{Over $\mathbb{F}_{2}$ With the First Four}

\section{Coefficients Prescribed}

In this chapter we study the number of irreducible polynomials

$$
f(x)=x^{n}+a_{n-1} x^{n-1}+\cdots+a_{0} \in \mathbb{F}_{2}[x]
$$

where $n$ is even and the four coefficients of $a_{n-1}, a_{n-2}, a_{n-3}$ and $a_{n-4}$ are given. Section 5.1 gives the previous results and the necessary background for this chapter. In Section 5.2 we give the formula for the number $F\left(n, t_{1}, t_{2}, t_{3}, t_{4}\right)$, which is the number of element $\beta \in \mathbb{F}_{2^{n}}$ where $T_{i}(\beta)=t_{i} \in \mathbb{F}_{2}$, and $i=1,2,3,4$. The main result of this chapter is the formula for $N\left(n, t_{1}, t_{2}, t_{3}, t_{4}\right)$, which is the number of irreducible polynomials $f$ of even degree $n$ with prescribed traces $T_{i}(f)=t_{i}, i=1,2,3,4$ given in 
Section 5.3. We use the generalization of Möbius inversion formula given in Chapter 3, to connect this number to $F\left(n, t_{1}, t_{2}, t_{3}, t_{4}\right)$.

\subsection{Preliminary Results and Background}

Let $P\left(n, t_{1}, t_{2}, \ldots, t_{r}, 2\right)$ be the set of irreducible polynomials $f \in \mathbb{F}_{2}[x]$ of degree $n$ with given $i^{t h}$ trace as $T_{i}(f)=t_{i} \in \mathbb{F}_{2}$, for $i=1,2, \ldots, r$. Since here we are limited to the finite field $\mathbb{F}_{2}$, so to enhance the readability we replace $P\left(n, t_{1}, t_{2}, \ldots, t_{r}, 2\right)$ by $P\left(n, t_{1}, t_{2}, \ldots, t_{r}\right)$. Let $N\left(n, t_{1}, t_{2}, \ldots, t_{r}\right)$ be the number of polynomials in the set $P\left(n, t_{1}, t_{2}, \ldots, t_{r}\right)$. Also let $P(n)$ be the set of all irreducible polynomials $f$ of degree $n$ over $\mathbb{F}_{2}$, and $N(n)=|P(n)|$. If in Equation (3.1) we let $q=2$, then we have

$$
N(n)=\frac{1}{n} \sum_{d \mid n} \mu(d) 2^{\frac{n}{d}}
$$

By this notation $N\left(n, t_{1}\right)$ is the number of irreducible polynomials $f$ of degree $n$ over $\mathbb{F}_{2}$ with given trace $T_{1}(f)=t_{1} \in \mathbb{F}_{2}$. If $n$ is a positive even integer, then it is less well-known that

$$
N(n, 1)=\frac{1}{2 n} \sum_{\substack{d \mid n \\ d \text { odd }}} \mu(d) 2^{\frac{n}{d}} .
$$

Therefore

$$
N(n, 0)=N(n)-\frac{1}{2 n} \sum_{\substack{d \mid n \\ d \text { odd }}} \mu(d) 2^{\frac{n}{d}} .
$$

The following property of $N(n, 1)$ is proved in [3]. 
Lemma 40. Let $a \equiv b(\bmod 4)$ be shortened to $a \equiv b$. Then,

$$
\sum_{\substack{d \mid n \\ d \equiv 2}} \frac{n}{d} N(n / d, 1)=\sum_{\substack{d \mid n \\ d \equiv 0}} \frac{n}{d} N(n / d)+\sum_{\substack{d \mid n \\ d \equiv 2}} \frac{n}{d} N(n / d, 0)= \begin{cases}2^{\frac{n}{2}-1} & \text { if } n \text { even } \\ 0 & \text { if } n \text { odd }\end{cases}
$$

We use [.] to rewrite Lemma 40:

$$
\sum_{\substack{d \mid n \\ d \equiv 2}} \frac{n}{d} N(n / d, 1)=\sum_{\substack{d \mid n \\ d \equiv 0}} \frac{n}{d} N(n / d)+\sum_{\substack{d \mid n \\ d \equiv 2}} \frac{n}{d} N(n / d, 0)=[n \text { even }] 2^{\frac{n}{2}-1}
$$

In the following we prove some lemmas about the numbers $N(n), N(n, 0)$ and $N(n, 1)$ which later in Section 5.3 we use to prove the main result of this chapter.

Lemma 41. Let $n$ be a given positive even integer. Then

$$
\sum_{\substack{d \mid n \\ d=0}} \frac{n}{d} N(n / d)= \begin{cases}2^{\frac{n}{4}} & \text { if } n=4 l \text { for some } l \\ 0 & \text { otherwise }\end{cases}
$$

where $d \equiv 0$ is the shortened form of $d \equiv 0(\bmod 4)$.

Proof. Let $n$ be a positive even integer, and $d \equiv 0(\bmod 4)$ be shortened as $d \equiv 0$. By Equation(5.1) we have

$$
\sum_{\substack{d \mid n \\ d \equiv 0}} \frac{n}{d} N(n / d)=\sum_{\substack{d \mid n \\ d=0}} \frac{n}{d}\left(\frac{d}{n} \sum_{k \mid \frac{n}{d}} \mu(k) 2^{\frac{n}{d k}}\right)=\sum_{\substack{d \mid n \\ d \equiv 0}} \sum_{k d \mid n} \mu(k) 2^{\frac{n}{d k}}
$$

Since $k d \mid n$, we have $n=(k d) \alpha$, for some $\alpha$. So $n=d(k \alpha)$ which implies $d \mid n$. Therefore the condition $k d \mid n$ satisfies $d \mid n$, which means we can consider the double 
sum as a single sum. Then

$$
\sum_{\substack{d \mid n \\ d \equiv 0}} \frac{n}{d} N(n / d)=\sum_{\substack{k d \mid n \\ d \equiv 0}} \mu(k) 2^{\frac{n}{d k}}
$$

Let $k d=m$, or $d=\frac{m}{k}$. Then $d \equiv 0$ means $\frac{m}{k} \equiv 0$ or $\frac{m}{k}=4 \theta$, for some $\theta$. Then $m=4 k \theta$ which implies $k \mid \frac{m}{4}$. Thus

$$
\begin{aligned}
\sum_{\substack{d \mid n \\
d=0}} \frac{n}{d} N(n / d) & =\sum_{\substack{m \mid n \\
d=0}} \mu(k) 2^{\frac{n}{m}}=\sum_{\substack{m \mid n \\
\frac{m}{k} \equiv 0}} \mu(k) 2^{\frac{n}{m}}=\sum_{\substack{m|n, k| \frac{m}{4} \\
m=0}} \mu(k) 2^{\frac{n}{m}} \\
& =\sum_{\substack{m \mid n \\
m \equiv 0}} 2^{\frac{n}{m}} \sum_{k \mid \frac{m}{4}} \mu(k) .
\end{aligned}
$$

If $\frac{m}{4}=1$ then we know that

$$
\sum_{k \mid \frac{m}{4}} \mu(k)=\sum_{k \mid 1} \mu(k)=\mu(1)=1
$$

Otherwise, for any $m$ where $m \equiv 0(\bmod 4)$ the sum is zero. Therefore the only remaining case is $m=4$. Since $k \mid \frac{m}{4}$, then $k \mid 1$, or $k=1$. This means $d=\frac{m}{k}=4$. Then $d \mid n$ implies $4 \mid n$ or $n=4 l$, for some $l$. So

$$
\sum_{\substack{d \mid n \\ d \equiv 0}} \frac{n}{d} N(n / d)=[n=4 l] 2^{\frac{n}{4}},
$$

which ends the proof.

Lemma 42. Let $n$ be a given positive even integer, and $a \equiv b(\bmod 8)$ be shortened 
as $a \equiv b$. Then

$$
\sum_{\substack{d \mid n \\ d \equiv 4}} \frac{n}{d} N(n / d, 1)= \begin{cases}2^{\frac{n}{4}-1} & \text { if } n=4 l \text { for some integer } l \\ 0 & \text { otherwise }\end{cases}
$$

Proof. Since in the sum $d \equiv 4(\bmod 8)$ we have $d=8 k+4=2(4 k+2)$, for some integer $k$. Then $4 k+2=\frac{d}{2}=d^{\prime}$ which implies $d^{\prime} \equiv 2(\bmod 4)$, or briefly $d^{\prime} \equiv 2$. Moreover, from $d \mid n$ we have $n=d c$, for some $c$, or $n=2 d^{\prime} c=2\left(d^{\prime} c\right)$. Therefore $\frac{n}{2}=c d^{\prime}$, or $d^{\prime} \mid \frac{n}{2}$. Let $\frac{n}{2}=n^{\prime}$. Then the sum can be written as

$$
\sum_{\substack{d \mid n \\ d \equiv 4}} \frac{n}{d} N(n / d, 1)=\sum_{\substack{d^{\prime} \mid{ }^{\prime} \\ d^{\prime} \equiv 2}} \frac{n^{\prime}}{d^{\prime}} N\left(n^{\prime} / d^{\prime}, 1\right)
$$

where in the last sum $d^{\prime} \equiv 2$ denotes $d^{\prime} \equiv 2(\bmod 4)$. Then by Lemma 40 if $n^{\prime}$ is even this sum is $2^{\frac{n^{\prime}}{2}-1}$, and otherwise it is zero. Since $\frac{n}{2}=n^{\prime}=2 l$ for some $l$, we have $n=4 l$. Thus,

$$
\sum_{\substack{d \mid n \\ d \equiv 4}} \frac{n}{d} N(n / d, 1)=[n=4 l] 2^{\frac{n}{4}-1}
$$

Consider $F\left(n, t_{1}, t_{2}, \ldots, t_{r}\right)$ as the number of elements $\beta \in \mathbb{F}_{2^{n}}$ with given $i^{\text {th }}$ trace as $T_{i}(\beta)=t_{i} \in \mathbb{F}_{2}$ for $i=1,2, \ldots, r$. Therefore

$$
F\left(n, t_{1}, t_{2}\right)=\left|\left\{\beta \in \mathbb{F}_{2^{n}}: T_{1}(\beta)=t_{1}, T_{2}(\beta)=t_{2}\right\}\right|
$$

In a similar way,

$$
N\left(n, t_{1}, t_{2}\right)=\left|\left\{f \in \mathbb{F}_{2}[x]: f \in N(n), T_{i}(f)=t_{i}, i=1,2\right\}\right| .
$$


In [3], a generalization of Möbius inversion formula given in Theorem 21 is used to find the numbers $N\left(n, t_{1}, t_{2}\right)$ in terms of the numbers $F\left(n, t_{1}, t_{2}\right)$.

Theorem 43. [3] Let $n$ be an even integer. Assume that $a \equiv b(\bmod 4)$ is shortened to $a \equiv b$. Then

(i) $F(n, 0,0)=[n$ even $] 2^{\frac{n}{2}-1}+\sum_{\substack{d \mid n \\ d \text { odd }}} \frac{n}{d} N(n / d, 0,0)$,

(ii) $F(n, 0,1)=[n$ even $] 2^{\frac{n}{2}-1}+\sum_{\substack{d \mid n \\ d \text { odd }}} \frac{n}{d} N(n / d, 0,1)$

(iii) $F(n, 1,0)=\sum_{\substack{d \mid n \\ d \equiv 1}} \frac{n}{d} N(n / d, 1,0)+\sum_{\substack{d \mid n \\ d \equiv 3}} \frac{n}{d} N(n / d, 1,1)$,

(iv) $F(n, 1,1)=\sum_{\substack{d \mid n \\ d \equiv 1}} \frac{n}{d} N(n / d, 1,1)+\sum_{\substack{d \mid n \\ d \equiv 3}} \frac{n}{d} N(n / d, 1,0)$,

if and only if

(v) $n N(n, 0,0)=\sum_{\begin{array}{c}d \mid n \\ d \text { odd }\end{array}} \mu(d) F(n / d, 0,0)-\sum_{\begin{array}{c}\text { ann, 足 even } \\ \text { odd }\end{array}} \mu(d) 2^{\frac{n}{2 d}-1}$

(vi) $n N(n, 0,1)=\sum_{\begin{array}{c}d \mid n \\ d \text { odd }\end{array}} \mu(d) F(n / d, 0,1)-\sum_{\begin{array}{c}\text { d|n, } \\ \text { d o even }\end{array}} \mu(d) 2^{\frac{n}{2 d}-1}$,

(vii) $n N(n, 1,0)=\sum_{\substack{d \mid n \\ d \equiv 1}} \mu(d) F(n / d, 1,0)+\sum_{\substack{d \mid n \\ d \equiv 3}} \mu(d) F(n / d, 1,1)$,

(viii) $n N(n, 1,1)=\sum_{\substack{d \mid n \\ d \equiv 1}} \mu(d) F(n / d, 1,1)+\sum_{\substack{d \mid n \\ d \equiv 3}} \mu(d) F(n / d, 1,0)$.

Moreover the numbers $F\left(n, t_{1}, t_{2}\right)$ are given in the following theorem.

Theorem 44. [3] If $n=2 m \geq 2$, then $F\left(n, t_{1}, t_{2}\right)=2^{n-2}+G\left(n, t_{1}, t_{2}\right)$, such that $G\left(n, t_{1}, t_{2}\right)=0$ or $\pm 2^{m-1}$, depending on $m$ as given in Table 5.1 . 
Table 5.1: Values of $G\left(n, t_{1}, t_{2}\right)$.

\begin{tabular}{|c|c|c|c|c|}
\hline$m(\bmod 4) \quad\left(t_{1}, t_{2}\right)$ & $(0,0)$ & $(0,1)$ & $(1,0)$ & $(1,1)$ \\
\hline 0 & $-2^{m-1}$ & $2^{m-1}$ & 0 & 0 \\
\hline 1 & 0 & 0 & $-2^{m-1}$ & $2^{m-1}$ \\
\hline 2 & $2^{m-1}$ & $-2^{m-1}$ & 0 & 0 \\
\hline 3 & 0 & 0 & $2^{m-1}$ & $-2^{m-1}$ \\
\hline
\end{tabular}

If $n$ is odd then [3] has a similar result. Although for our studies we do not need the result $N\left(n, t_{1}, t_{2}, t_{3}\right)$, for completeness we give them here. Let $f$ be a polynomial defined in $\mathbb{F}_{2}[x]$. If $t_{1}, t_{2}$ and $t_{3}$ are given as the first three traces of $f$, and $n$ is an even number, then in [24] the authors find the number $N\left(n, t_{1}, t_{2}, t_{3}\right)$ by connecting it to the number $F\left(n, t_{1}, t_{2}, t_{3}\right)$.

Theorem 45. Let $n$ be even and $a \equiv b(\bmod 4)$ be shortened $a s a \equiv b$. Then

$$
\begin{aligned}
& n N(n, 1,0,0)=\sum_{\substack{d \mid n \\
d \equiv 1}} \mu(d) F(n / d, 1,0,0)+\sum_{\substack{d \mid n \\
d \equiv 3}} \mu(d) F(n / d, 1,1,1), \\
& n N(n, 1,0,1)=\sum_{\substack{d \mid n \\
d \equiv 1}} \mu(d) F(n / d, 1,0,1)+\sum_{\substack{d \mid n \\
d \equiv 3}} \mu(d) F(n / d, 1,1,0), \\
& n N(n, 1,1,0)=\sum_{\substack{d \mid n \\
d \equiv 1}} \mu(d) F(n / d, 1,1,0)+\sum_{\substack{d \mid n \\
d \equiv 3}} \mu(d) F(n / d, 1,0,1), \\
& n N(n, 1,1,1)=\sum_{\substack{d \mid n \\
d \equiv 1}} \mu(d) F(n / d, 1,1,1)+\sum_{\substack{d \mid n \\
d \equiv 3}} \mu(d) F(n / d, 1,0,0), \\
& n N(n, 0,0,0)=\sum_{\substack{d \mid n \\
d \text { odd }}} \mu(d) F(n / d, 0,0,0)-\sum_{\substack{d \mid n, n \text { even } \\
d \text { odd }}} \mu(d) 2^{\frac{n}{2 d}-1} \\
& n N(n, 0,1,0)=\sum_{\substack{d \mid n \\
d \text { odd }}} \mu(d) F(n / d, 0,1,0)-\sum_{\begin{array}{c}
d \mid n, \frac{n}{d} \text { even } \\
d \text { odd }
\end{array}} \mu(d) 2^{\frac{n}{2 d}-1} \\
& n N(n, 0,0,1)=\sum_{\substack{d \mid n \\
d \text { odd }}} \mu(d) F(n / d, 0,0,1) \\
& n N(n, 0,1,1)=\sum_{\substack{d \mid n \\
d \text { odd }}} \mu(d) F(n / d, 0,1,1)
\end{aligned}
$$


Moreover, if $n=2 m \geq 3$, then

$$
F\left(n, t_{1}, t_{2}, t_{3}\right)=2^{n-3}+G\left(n, t_{1}, t_{2}, t_{3}\right),
$$

such that the number $G\left(n, t_{1}, t_{2}, t_{3}\right)$ is given in Tables 5.2 and 5.3.

Table 5.2: Values of $G\left(n, t_{1}, t_{2}, t_{3}\right)$.

\begin{tabular}{|c|c|c|c|c|}
\hline$m(\bmod 12)$ & $(0,0,0)$ & $(0,0,1)$ & $(0,1,0)$ & $(0,1,1)$ \\
\hline 0 & $-2^{m}-2^{m-2}$ & $2^{m-1}+2^{m-2}$ & $2^{m-2}$ & $2^{m-2}$ \\
\hline 1 or 5 & $2^{m-2}$ & $-2^{m-2}$ & $2^{m-2}$ & $-2^{m-2}$ \\
\hline 2 or 10 & 0 & $2^{m-1}$ & 0 & $-2^{m-1}$ \\
\hline 3 & $2^{m-2}$ & $-2^{m-2}$ & $2^{m-2}$ & $-2^{m-2}$ \\
\hline 4 or 8 & $-2^{m-1}$ & 0 & $-2^{m-1}$ & $2^{m}$ \\
\hline 6 & $2^{m-1}+2^{m-2}$ & $-2^{m-2}$ & $-2^{m-1}-2^{m-2}$ & $2^{m-2}$ \\
\hline 7 or 11 & $2^{m-2}$ & $-2^{m-2}$ & $2^{m-2}$ & $-2^{m-2}$ \\
\hline 9 & $2^{m-2}$ & $-2^{m-2}$ & $2^{m-2}$ & $-2^{m-2}$ \\
\hline
\end{tabular}

Table 5.3: Values of $G\left(n, t_{1}, t_{2}, t_{3}\right)$ (Cont.).

\begin{tabular}{|c|c|c|c|c|}
\hline$m(\bmod 12) \quad\left(t_{1}, t_{2}, t_{3}\right)$ & $(1,0,0)$ & $(1,0,1)$ & $(1,1,0)$ & $(1,1,1)$ \\
\hline 0 & $\overline{0}$ & 0 & 0 & 0 \\
\hline 1 or 5 & $-2^{m-2}$ & $-2^{m-2}$ & $-2^{m-2}$ & $2^{m-1}+2^{m-2}$ \\
\hline 2 or 10 & $2^{m-1}$ & $-2^{m-1}$ & $-2^{m-1}$ & $-2^{m-1}$ \\
\hline 3 & $-2^{m-2}$ & $2^{m-1}$ & 0 & $2^{m-1}$ \\
\hline 4 or 8 & 0 & 0 & 0 & 0 \\
\hline 6 & $2^{m-1}$ & $-2^{m-1}$ & $-2^{m-1}$ & $-2^{m-1}$ \\
\hline 7 or 11 & $-2^{m-2}$ & $2^{m-1}+2^{m-2}$ & $-2^{m-2}$ & $-2^{m-2}$ \\
\hline 9 & $-2^{m}$ & $2^{m-1}$ & 0 & $2^{m-1}$ \\
\hline
\end{tabular}

For the odd case of $n$ the formulas for $N\left(n, t_{1}, t_{2}, t_{3}\right)$ are given in [7]. Our plan is to use a similar methodology as before, but for polynomials with the four coefficients $a_{n-1}, a_{n-2}, a_{n-3}$ and $a_{n-4}$ prescribed. 


\subsection{The Formula For $F\left(n, t_{1}, t_{2}, t_{3}, t_{4}\right)$}

Let $F\left(n, t_{1}, t_{2}, t_{3}, t_{4}\right)$ be the number of elements $\beta \in \mathbb{F}_{2^{n}}$ with $T_{i}(\beta)=t_{i}$ where $i=1,2,3,4$. In this section we study $F\left(n, t_{1}, t_{2}, t_{3}, t_{4}\right)$. Let $f=\operatorname{Min}_{\beta} \in \mathbb{F}_{2}[x]$ of degree $n / d$ be the minimal polynomial of $\beta \in \mathbb{F}_{2^{n}}$. The following lemma regarding the connection between the traces of $\beta \in \mathbb{F}_{2^{n}}$ and the coefficients of $f^{d}$ is proved in [3].

Lemma 46. Let $f \in \mathbb{F}_{2}[x]$ of degree $n / d$ be the minimal polynomial of $\beta \in \mathbb{F}_{2^{n}}$. Then for $1 \leq i \leq n$ the $i^{\text {th }}$ trace of $\beta$ is the $i^{\text {th }}$ trace of $f^{d}$, which is the coefficient of $x^{n-i}$ in $f^{d}$, that is, $T_{i}(\beta)=T_{i}\left(f^{d}\right)$, where $i=1, \ldots, n$.

For a given polynomial $f \in \mathbb{F}_{2}[x]$ the following proposition gives the connection between the coefficients of the polynomials $f$ and $f^{d}$, for all positive integers $d$.

Proposition 47. Let $d \geq 1$ be an integer, and $f \in \mathbb{F}_{2}[x]$. Then

(i) $T_{1}\left(f^{d}\right)=d T_{1}(f)$

(ii) $T_{2}\left(f^{d}\right)=\left(\begin{array}{l}d \\ 2\end{array}\right) T_{1}(f)+d T_{2}(f)$;

(iii) $T_{3}\left(f^{d}\right)=\left(\begin{array}{l}d \\ 3\end{array}\right) T_{1}(f)+d T_{3}(f)$

(iv) $T_{4}\left(f^{d}\right)=\left(\begin{array}{l}d \\ 4\end{array}\right) T_{1}(f)+\left(\begin{array}{l}d \\ 2\end{array}\right) T_{2}(f)+d T_{4}(f)$.

Proof. Let $f \in \mathbb{F}_{2}[x]$ be given such that $\operatorname{deg}(f)=n$, and

$$
f(x)=x^{n}+a_{n-1} x^{n-1}+a_{n-2} x^{n-2}+\cdots+a_{1} x+a_{0} .
$$


Then by the multinomial theorem (see Theorem 16) we have

$$
\begin{aligned}
(f(x))^{d} & =\left(x^{n}+a_{n-1} x^{n-1}+\cdots+a_{1} x+a_{0}\right)^{d} \\
& =\sum_{i_{0}+\cdots+i_{n}=d} \frac{d !}{i_{0} ! i_{1} ! \cdots i_{n} !}\left(x^{n}\right)^{i_{0}}\left(a_{n-1} x^{n-1}\right)^{i_{1}} \cdots\left(a_{1} x\right)^{i_{n-1}}\left(a_{0}\right)^{i_{n}} \\
& =\sum_{i_{0}+\cdots+i_{n}=d} \frac{d !\left(a_{n-1}^{i_{1}} \cdots a_{1}^{i_{n-1}} a_{0}^{i_{n}}\right)}{i_{0} ! i_{1} ! \cdots i_{n} !}\left(x^{n i_{0}+(n-1) i_{1}+\cdots+i_{n-1}}\right) .
\end{aligned}
$$

If in $(f(x))^{d}$ we let $i_{0}=d$ and $i_{1}=i_{2}=\cdots=i_{n}=0$, then clearly $i_{0}+\cdots+i_{n}=d$, and the sum gives the term $x^{\text {nd }}$ which has coefficient

$$
\frac{d !}{i_{0} ! i_{1} ! \cdots i_{n} !}=\frac{d !}{d !}=1
$$

(i) We know that $T_{1}\left(f^{d}\right)$ is the coefficient of $x^{n d-1}$ in the polynomial $f^{d}$. To find this coefficient, in the previous sum we let $i_{0}=d-1, i_{1}=1$ and $i_{k}=0$, for $k=2,3, \ldots, n$. Then

$$
n i_{0}+(n-1) i_{1}+\cdots+i_{n-1}=(d-1) n+n-1=n d-1 .
$$

So the coefficient of $x^{n d-1}$ is

$$
T_{1}\left(f^{d}\right)=\frac{d !}{(d-1) ! 1 !} a_{n-1}=d a_{n-1}=d T_{1}(f)
$$

(ii) We know that $T_{2}\left(f^{d}\right)$ is the coefficient of $x^{n d-2}$ in the polynomial $f^{d}$. Since we want $n i_{0}+(n-1) i_{1}+\cdots+i_{n-1}=n d-2$ so we choose $i_{0}, i_{1}, \ldots, i_{n}$ such that either Case $(i) i_{0}=d-2, i_{1}=2$ and $i_{2}=\cdots=i_{n}=0$, or Case $(i i) i_{0}=d-1, i_{2}=1$ and $i_{1}=i_{3}=\cdots=i_{n}=0$. Then in Case $(i)$ the coefficient 
of the term $x^{n d-2}$ is

$$
\frac{d !}{(d-2) ! 2 !} a_{n-1}^{2}=\left(\begin{array}{l}
d \\
2
\end{array}\right) a_{n-1}^{2}=\left(\begin{array}{l}
d \\
2
\end{array}\right) a_{n-1}=\left(\begin{array}{l}
d \\
2
\end{array}\right) T_{1}(f) .
$$

We used $f(x)=x^{n}+a_{n-1} x^{n-1}+a_{n-2} x^{n-2}+\cdots+a_{1} x+a_{0} \in \mathbb{F}_{2}$ and so $a_{i}{ }^{2}=a_{i}$ for all $0 \leq i \leq n-1$. In a similar way in Case $(i i)$ the coefficient of $x^{n d-2}$ is

$$
\frac{d !}{(d-1) ! 1 !} a_{n-2}=\left(\begin{array}{l}
d \\
1
\end{array}\right) a_{n-2}=d a_{n-2}=d T_{2}(f) .
$$

Thus the coefficient of $x^{n d-2}$ in $f^{d}$ is

$$
T_{2}\left(f^{d}\right)=\left(\begin{array}{l}
d \\
2
\end{array}\right) T_{1}(f)+d T_{2}(f)
$$

(iii) In this part we compute the coefficient of $x^{n d-3}$ in $f^{d}$. Here we need

$$
n i_{0}+(n-1) i_{1}+\cdots+i_{n-1}=n d-3
$$

which is possible when either

Case $(i) i_{0}=d-3, i_{1}=3$ and $i_{2}=i_{3}=\cdots=i_{n}=0$, or

Case $(i i) i_{0}=d-1, i_{3}=1$ and $i_{1}=i_{2}=i_{4}=\cdots=i_{n}=0$.

Then from Case $(i)$ the coefficient of $x^{n d-3}$ is $\left(\begin{array}{l}d \\ 3\end{array}\right) a_{n-1}=\left(\begin{array}{l}d \\ 3\end{array}\right) T_{1}(f)$, and from Case $(i i)$ the coefficient of $x^{n d-3}$ is $d a_{n-3}=d T_{3}(f)$. Therefore

$$
T_{3}\left(f^{d}\right)=\left(\begin{array}{l}
d \\
3
\end{array}\right) T_{1}(f)+d T_{3}(f)
$$

(iv) To find $T_{4}\left(f^{d}\right)$, that is, the coefficient of $x^{n d-4}$ in $f^{d}$, we choose $i_{0}, i_{1}, \ldots, i_{n}$ such that $n i_{0}+(n-1) i_{1}+\cdots+i_{n-1}=n d-4$. This is possible in one the following cases: 
Case $(i) i_{0}=d-4, i_{1}=4$ and $i_{2}=i_{3}=\cdots=i_{n}=0$. Then the coefficient of $x^{n d-4}$ is

$$
\left(\begin{array}{l}
d \\
4
\end{array}\right) a_{n-4}=\left(\begin{array}{l}
d \\
4
\end{array}\right) T_{1}(f)
$$

Case $(i i) i_{0}=d-2, i_{2}=2$ and $i_{1}=i_{3}=i_{4}=\cdots=i_{n}=0$. Then the coefficient is

$$
\left(\begin{array}{l}
d \\
2
\end{array}\right) a_{n-2}^{2}=\left(\begin{array}{l}
d \\
2
\end{array}\right) a_{n-2}=\left(\begin{array}{l}
d \\
2
\end{array}\right) T_{2}(f)
$$

Case $(i i i) i_{0}=d-1, i_{4}=1$ and $i_{1}=i_{2}=i_{3}=\cdots=i_{n}=0$. So the coefficient is $d a_{n-4}=d T_{4}(f)$. This implies,

$$
T_{4}\left(f^{d}\right)=\left(\begin{array}{l}
d \\
4
\end{array}\right) T_{1}(f)+\left(\begin{array}{l}
d \\
2
\end{array}\right) T_{2}(f)+d T_{4}(f)
$$

Proposition 48. Let $d$ be a given integer such that $d \geq 1$. Then we have the following situations.

(i) If $d \equiv 0(\bmod 8)$ then $d$ is even, and

$$
\left(\begin{array}{l}
d \\
2
\end{array}\right) \equiv\left(\begin{array}{l}
d \\
3
\end{array}\right) \equiv\left(\begin{array}{l}
d \\
4
\end{array}\right) \equiv 0 \quad(\bmod 2)
$$

Therefore

$$
T_{i}\left(f^{d}\right)=0, \quad i=1,2,3,4 .
$$

(ii) If $d \equiv 1(\bmod 8)$ then $d$ is odd, and

$$
\left(\begin{array}{l}
d \\
2
\end{array}\right) \equiv\left(\begin{array}{l}
d \\
3
\end{array}\right) \equiv\left(\begin{array}{l}
d \\
4
\end{array}\right) \equiv 0 \quad(\bmod 2)
$$


Thus,

$$
T_{i}\left(f^{d}\right)=T_{i}(f), \quad i=1,2,3,4
$$

(iii) If $d \equiv 2(\bmod 8)$ then $d$ is even, and

$$
\left(\begin{array}{l}
d \\
2
\end{array}\right) \equiv 1, \quad\left(\begin{array}{l}
d \\
3
\end{array}\right) \equiv\left(\begin{array}{l}
d \\
4
\end{array}\right) \equiv 0 \quad(\bmod 2)
$$

Then,

$$
T_{1}\left(f^{d}\right)=T_{3}\left(f^{d}\right)=0, \quad T_{2}\left(f^{d}\right)=T_{1}(f), \quad T_{4}\left(f^{d}\right)=T_{2}(f)
$$

(iv) If $d \equiv 3(\bmod 8)$ then $d$ is odd, and

$$
\left(\begin{array}{l}
d \\
2
\end{array}\right) \equiv\left(\begin{array}{l}
d \\
3
\end{array}\right) \equiv 1, \quad\left(\begin{array}{l}
d \\
4
\end{array}\right) \equiv 0 \quad(\bmod 2)
$$

Moreover, $T_{\mathbf{1}}\left(f^{d}\right)=T_{1}(f)$ and

$$
\begin{aligned}
& T_{2}\left(f^{d}\right)=T_{1}(f)+T_{2}(f), \\
& T_{3}\left(f^{d}\right)=T_{1}(f)+T_{3}(f), \\
& T_{4}\left(f^{d}\right)=T_{2}(f)+T_{4}(f) .
\end{aligned}
$$

(v) If $d \equiv 4(\bmod 8)$ then $d$ is even, and

$$
\left(\begin{array}{l}
d \\
2
\end{array}\right) \equiv\left(\begin{array}{l}
d \\
3
\end{array}\right) \equiv 0, \quad\left(\begin{array}{l}
d \\
4
\end{array}\right) \equiv 1 \quad(\bmod 2)
$$

Also $T_{i}\left(f^{d}\right)=0$, for $i=1,2,3$, and $T_{4}\left(f^{d}\right)=T_{1}(f)$.

(vi) If $d \equiv 5(\bmod 8)$, then $d$ is odd, and

$$
\left(\begin{array}{l}
d \\
2
\end{array}\right) \equiv\left(\begin{array}{l}
d \\
3
\end{array}\right) \equiv 0, \quad\left(\begin{array}{l}
d \\
4
\end{array}\right) \equiv 1 \quad(\bmod 2)
$$


Then $T_{i}\left(f^{d}\right)=T_{i}(f)$, for $i=1,2,3$, and $T_{4}\left(f^{d}\right)=T_{1}(f)+T_{4}(f)$.

(vii) If $d \equiv 6(\bmod 8)$ then $d$ is even, and

$$
\left(\begin{array}{l}
d \\
2
\end{array}\right) \equiv\left(\begin{array}{l}
d \\
4
\end{array}\right) \equiv 1, \quad\left(\begin{array}{l}
d \\
3
\end{array}\right) \equiv 0 \quad(\bmod 2)
$$

In addition, $T_{1}\left(f^{d}\right)=T_{3}\left(f^{d}\right)=0, T_{2}\left(f^{d}\right)=T_{1}(f)$, and

$$
T_{4}\left(f^{d}\right)=T_{1}(f)+T_{2}(f)
$$

(viii) If $d \equiv 7(\bmod 8)$ then $d$ is odd, and

$$
\left(\begin{array}{l}
d \\
2
\end{array}\right) \equiv\left(\begin{array}{l}
d \\
3
\end{array}\right) \equiv\left(\begin{array}{l}
d \\
4
\end{array}\right) \equiv 1 \quad(\bmod 2)
$$

Therefore $T_{1}\left(f^{d}\right)=T_{1}(f)$ and

$$
\begin{aligned}
& T_{2}\left(f^{d}\right)=T_{1}(f)+T_{2}(f), \\
& T_{3}\left(f^{d}\right)=T_{1}(f)+T_{3}(f), \\
& T_{4}\left(f^{d}\right)=T_{1}(f)+T_{2}(f)+T_{4}(f) .
\end{aligned}
$$

Proof. Let $d$ be a positive integer.

(i) If $d \equiv 0(\bmod 8)$ then $d=8 k$, for some $k$. So $d$ is even, and

$$
\left(\begin{array}{l}
d \\
2
\end{array}\right)=\frac{d(d-1)}{2}=\frac{8 k(8 k-1)}{2}=4 k(8 k-1),
$$


which is even. Therefore $d \equiv\left(\begin{array}{l}d \\ 2\end{array}\right) \equiv 0(\bmod 2)$. Also

$$
\begin{aligned}
& \left(\begin{array}{l}
d \\
3
\end{array}\right)=\frac{d(d-1)(d-2)}{6}=\frac{4 k(8 k-2)(8 k-1)}{3} \\
& \left(\begin{array}{l}
d \\
4
\end{array}\right)=\frac{d(d-1)(d-2)(d-3)}{24}=\frac{k(8 k-2)(8 k-1)(8 k-3)}{3},
\end{aligned}
$$

which both are even, and therefore are zeros. Then by Proposition 47 we have

$$
T_{1}\left(f^{d}\right)=T_{2}\left(f^{d}\right)=T_{3}\left(f^{d}\right)=T_{4}\left(f^{d}\right)=0 .
$$

The proofs of the other cases are similar.

We recall that $P(n)$ is the set of all irreducible polynomials of degree $n$ over $\mathbb{F}_{2}$. By a.P(n) we denote the multiset that contains $a$ copies of the set $P(n)$. The following lemma gives a formula for the number $F\left(n, t_{1}, t_{2}, t_{3}, t_{4}\right)$.

Lemma 49. Let $n$ be an even positive integer and $t_{i} \in \mathbb{F}_{2}$, for $1 \leq i \leq 4$. Also let $a \equiv b(\bmod 8)$ be shortened as $a \equiv b$. Then the number of elements $\beta \in \mathbb{F}_{2^{n}}$ with prescribed first four traces $T_{i}(\beta)=t_{i}$ is

$$
F\left(n, t_{1}, t_{2}, t_{3}, t_{4}\right)=\sum_{i=0}^{7} \bigcup_{\substack{d \mid n \\ d=i}} \frac{n}{d} \cdot\left|S_{i}\right|,
$$

where the sets $S_{0}, \ldots, S_{7}$ are defined as:

$$
\begin{aligned}
& S_{0}=\left\{f \in P(n / d): t_{i}=0, i=1,2,3,4\right\}, \\
& S_{1}=\left\{f \in P(n / d): T_{i}(f)=t_{i}, i=1,2,3,4\right\}, \\
& S_{2}=\left\{f \in P(n / d): t_{1}=t_{3}=0, T_{1}(f)=t_{2}, T_{2}(f)=t_{4}\right\},
\end{aligned}
$$




$$
\begin{aligned}
S_{3}= & \left\{f \in P(n / d): T_{1}(f)=t_{1}, T_{1}(f)+T_{2}(f)=t_{2},\right. \\
& \left.T_{1}(f)+T_{3}(f)=t_{3}, T_{2}(f)+T_{4}(f)=t_{4}\right\}, \\
S_{4}= & \left\{f \in P(n / d): t_{i}=0, i=1,2,3, T_{1}(f)=t_{4}\right\}, \\
S_{5}= & \left\{f \in P(n / d): T_{i}(f)=t_{i}, i=1,2,3, T_{1}(f)+T_{4}(f)=t_{4}\right\}, \\
S_{6}= & \left\{f \in P(n / d): t_{1}=t_{3}=0, T_{1}(f)=t_{2}, T_{1}(f)+T_{2}(f)=t_{4}\right\}, \\
S_{7}= & \left\{f \in P(n / d): T_{1}(f)=t_{1}, T_{1}(f)+T_{2}(f)=t_{2},\right. \\
& \left.T_{1}(f)+T_{3}(f)=t_{3}, T_{1}(f)+T_{2}(f)+T_{4}(f)=t_{4}\right\} .
\end{aligned}
$$

Proof. Let $f=\operatorname{Min}_{\beta}$ be the minimal polynomial of a given $\beta \in \mathbb{F}_{2^{n}}$. A classic result from finite field theory [14] imply the following equality about multisets:

$$
\bigcup_{\beta \in \mathbb{F}_{2 n}} \operatorname{Min}_{\beta}=\bigcup_{d \mid n} d \cdot P(d)=\bigcup_{d \mid n} \frac{n}{d} P\left(\frac{n}{d}\right)
$$

If in this equality we restrict the traces as $T_{i}(\beta)=t_{i}$, where $i=1,2,3,4$, then

$$
\begin{aligned}
F\left(n, t_{1}, t_{2}, t_{3}, t_{4}\right) & =\left|\bigcup_{\substack{\beta \in \mathbb{F}_{2} n \\
T_{i}(\beta)=t_{i}}} \operatorname{Min}_{\beta}\right| \\
& =\left|\bigcup_{d \mid n} \frac{n}{d} \cdot\left\{f \in P(n / d): T_{i}\left(f^{d}\right)=t_{i}, i=1,2,3,4\right\}\right| .
\end{aligned}
$$

By Proposition 48, for different values of $d=0, \ldots, 7$, each of the numbers $d,\left(\begin{array}{l}d \\ 2\end{array}\right),\left(\begin{array}{l}d \\ 3\end{array}\right)$ and $\left(\begin{array}{l}d \\ 4\end{array}\right)$ are either zero or one. Then using Proposition 47 we have different situations for $T_{i}\left(f^{d}\right)$, for $i=1,2,3,4$. For example, when $d \equiv 2(\bmod 8)$, Proposition $48(i i i)$ 
gives $\left(\begin{array}{l}d \\ 2\end{array}\right) \equiv 1(\bmod 2)$, while

$$
\left(\begin{array}{l}
d \\
3
\end{array}\right) \equiv\left(\begin{array}{l}
d \\
4
\end{array}\right) \equiv 0 \quad(\bmod 2)
$$

Then from $T_{i}\left(f^{d}\right)=t_{i}$, where $1 \leq i \leq 4$ and Proposition 47 we have

$$
\begin{aligned}
& t_{1}=T_{1}\left(f^{d}\right)=d T_{1}(f)=0, \\
& t_{2}=T_{2}\left(f^{d}\right)=\left(\begin{array}{l}
d \\
2
\end{array}\right) T_{1}(f)+d T_{2}(f)=T_{1}(f), \\
& t_{3}=T_{3}\left(f^{d}\right)=\left(\begin{array}{l}
d \\
3
\end{array}\right) T_{1}(f)+d T_{3}(f)=0, \\
& t_{4}=T_{4}\left(f^{d}\right)=\left(\begin{array}{l}
d \\
4
\end{array}\right) T_{1}(f)+\left(\begin{array}{l}
d \\
2
\end{array}\right) T_{2}(f)+d T_{4}(f)=T_{2}(f) .
\end{aligned}
$$

Thus for $d \equiv 2(\bmod 8)$ we define

$$
S_{2}=\left\{f \in P(n / d): t_{1}=t_{3}=0, T_{1}(f)=t_{2}, T_{2}(f)=t_{4}\right\} .
$$

For other values of $d$ we define corresponding sets as

$$
\begin{aligned}
S_{0}= & \left\{f \in P(n / d): t_{i}=0, i=1,2,3,4\right\}, \\
S_{1}= & \left\{f \in P(n / d): T_{i}(f)=t_{i}, i=1,2,3,4\right\}, \\
S_{3}= & \left\{f \in P(n / d): T_{1}(f)=t_{1}, T_{1}(f)+T_{2}(f)=t_{2},\right. \\
& \left.T_{1}(f)+T_{3}(f)=t_{3}, T_{2}(f)+T_{4}(f)=t_{4}\right\}, \\
S_{4}= & \left\{f \in P(n / d): t_{i}=0, i=1,2,3, T_{1}(f)=t_{4}\right\}, \\
S_{5}= & \left\{f \in P(n / d): T_{i}(f)=t_{i}, i=1,2,3, T_{1}(f)+T_{4}(f)=t_{4}\right\}, \\
S_{6}= & \left\{f \in P(n / d): t_{1}=t_{3}=0, T_{1}(f)=t_{2}, T_{1}(f)+T_{2}(f)=t_{4}\right\}, \\
S_{7}= & \left\{f \in P(n / d): T_{1}(f)=t_{1}, T_{1}(f)+T_{2}(f)=t_{2},\right.
\end{aligned}
$$




$$
\left.T_{1}(f)+T_{3}(f)=t_{3}, T_{1}(f)+T_{2}(f)+T_{4}(f)=t_{4}\right\}
$$

Then the formula for $F\left(n, t_{1}, t_{2}, t_{3}, t_{4}\right)$ can be given as

$$
F\left(n, t_{1}, t_{2}, t_{3}, t_{4}\right)=\left|\bigcup_{\substack{d \mid n \\ d \equiv 0}} \frac{n}{d} \cdot S_{0}\right|+\left|\bigcup_{\substack{d \mid n \\ d \equiv 1}} \frac{n}{d} \cdot S_{1}\right|+\cdots+\left|\bigcup_{\substack{d \mid n \\ d \equiv 7}} \frac{n}{d} \cdot S_{7}\right|
$$

Taking cardinality, we have the following formula for $F\left(n, t_{1}, t_{2}, t_{3}, t_{4}\right)$,

$$
F\left(n, t_{1}, t_{2}, t_{3}, t_{4}\right)=\sum_{\substack{d \mid n \\ d \equiv 0}} \frac{n}{d} \cdot\left|S_{0}\right|+\sum_{\substack{d \mid n \\ d \equiv 1}} \frac{n}{d} \cdot\left|S_{1}\right|+\cdots+\sum_{\substack{d \mid n \\ d \equiv 7}} \frac{n}{d} \cdot\left|S_{7}\right|,
$$

or

$$
F\left(n, t_{1}, t_{2}, t_{3}, t_{4}\right)=\sum_{i=0}^{7} \bigcup_{\substack{d \mid n \\ d \equiv i}} \frac{n}{d} \cdot\left|S_{i}\right|
$$

which ends the proof.

\subsection{The Formula For $N\left(n, t_{1}, t_{2}, t_{3}, t_{4}\right)$}

In this section we prove that $N\left(n, t_{1}, t_{2}, t_{3}, t_{4}\right)$ can be given in terms of $F\left(n, t_{1}, t_{2}, t_{3}, t_{4}\right)$. Suppose that $t_{i} \in \mathbb{F}_{2}$, where $i=1,2,3,4$. Then for different values of $t_{i}$ we have 16 cases for $\left(t_{1}, t_{2}, t_{3}, t_{4}\right)$. Since in some of these 16 cases the numbers $N\left(n, t_{1}, t_{2}, t_{3}, t_{4}\right)$ are connected to each other, we divide the 16 cases into 8 different groups. The following theorem, that is the main result of this chapter, gives $N\left(n, t_{1}, t_{2}, t_{3}, t_{4}\right)$ in terms of the numbers $F\left(n, t_{1}, t_{2}, t_{3}, t_{4}\right)$.

Theorem 50. Let $n$ be a given positive even integer and suppose $a \equiv b$ denotes $a \equiv b$ 
$(\bmod 8)$. Then the different values of $N\left(n, t_{1}, t_{2}, t_{3}, t_{4}\right)$ are given as

$$
\begin{aligned}
& \text { (i) } n N(n, 1,1,1,0)=\sum_{\substack{d \mid n \\
d=1}} \mu(d) F(n / d, 1,1,1,0)+\sum_{\substack{d \mid n \\
d=3}} \mu(d) F(n / d, 1,0,0,0) \\
& +\sum_{\substack{d \mid n \\
d \equiv 5}}^{d \equiv 1} \mu(d) F(n / d, 1,1,1,1)+\sum_{\substack{d \mid n \\
d \equiv 7}} \mu(d) F(n / d, 1,0,0,1) \\
& n N(n, 1,0,0,1)=\sum_{\substack{d \mid n \\
d=1}} \mu(d) F(n / d, 1,0,0,1)+\sum_{\substack{d \mid n \\
d \equiv 3}} \mu(d) F(n / d, 1,1,1,0) \\
& +\sum_{\substack{d \mid n \\
d \equiv 5}} \mu(d) F(n / d, 1,0,0,0)+\sum_{\substack{d \mid n \\
d \equiv 7}} \mu(d) F(n / d, 1,1,1,1), \\
& n N(n, 1,1,1,1)=\sum_{\substack{d \mid n \\
d \equiv=1}}^{d \equiv 5} \mu(d) F(n / d, 1,1,1,1)+\sum_{\substack{d \mid n \\
d \equiv=3}}^{d \equiv 7} \mu(d) F(n / d, 1,0,0,1) \\
& +\sum_{\substack{d \mid n \\
d \equiv 5}} \mu(d) F(n / d, 1,1,1,0)+\sum_{\substack{d \mid n \\
d \equiv 7}} \mu(d) F(n / d, 1,0,0,0) \\
& n N(n, 1,0,0,0)=\sum_{\substack{d \mid n \\
d \equiv 1}}^{d \equiv 5} \mu(d) F(n / d, 1,0,0,0)+\sum_{\substack{d \mid n \\
d \equiv 3}}^{d \equiv 7} \mu(d) F(n / d, 1,1,1,1) \\
& +\sum_{\substack{d \mid n \\
d \equiv 5}} \mu(d) F(n / d, 1,0,0,1)+\sum_{\substack{d \mid n \\
d \equiv 7}} \mu(d) F(n / d, 1,1,1,0), \\
& \text { (ii) } n N(n, 0,0,1,0)=\sum_{\substack{d \mid n \\
d \text { odd }}} \mu(d) F(n / d, 0,0,1,0) \\
& \text { (iii) } n N(n, 0,0,1,1)=\sum_{\begin{array}{c}
\text { d|n } \\
d \text { odd }
\end{array}} \mu(d) F(n / d, 0,0,1,1) \text {, } \\
& \text { (iv) } n N(n, 1,1,0,0)=\sum_{\substack{d \mid n \\
d \equiv 1}} \mu(d) F(n / d, 1,1,0,0)+\sum_{\substack{d \mid n \\
d \equiv 3}} \mu(d) F(n / d, 1,0,1,0) \\
& +\sum_{\substack{d \mid n \\
d=5}} \mu(d) F(n / d, 1,1,0,1)+\sum_{\substack{d \mid n \\
d \equiv 7}} \mu(d) F(n / d, 1,0,1,1) \\
& n N(n, 1,0,1,1)=\sum_{\substack{d \mid n \\
d=1}}^{d \equiv 5} \mu(d) F(n / d, 1,0,1,1)+\sum_{\substack{d \mid n \\
d \equiv 3}} \mu(d) F(n / d, 1,1,0,0) \\
& +\sum_{\substack{d \mid n \\
d=5}} \mu(d) F(n / d, 1,0,1,0)+\sum_{\substack{d \mid n \\
d \equiv 7}} \mu(d) F(n / d, 1,1,0,1),
\end{aligned}
$$




$$
\begin{aligned}
n N(n, 1,1,0,1) & =\sum_{\substack{d \mid n \\
d \equiv 1}} \mu(d) F(n / d, 1,1,0,1)+\sum_{\substack{d \mid n \\
d \equiv 3}} \mu(d) F(n / d, 1,0,1,1) \\
& +\sum_{\substack{d \mid n \\
d \equiv 5}} \mu(d) F(n / d, 1,1,0,0)+\sum_{\substack{d \mid n \\
d \equiv 7}} \mu(d) F(n / d, 1,0,1,0) \\
n N(n, 1,0,1,0)= & \sum_{d \mid n} \mu(d) F(n / d, 1,0,1,0)+\sum_{\substack{d \mid n \\
d \equiv 1}} \mu(d) F(n / d, 1,1,0,1) \\
& +\sum_{\substack{d \mid n \\
d \equiv 5}} \mu(d) F(n / d, 1,0,1,1)+\sum_{\substack{d \mid n \\
d \equiv 7}} \mu(d) F(n / d, 1,1,0,0)
\end{aligned}
$$

In the following cases, we use $a \equiv b$ to represent $a \equiv b(\bmod 4)$.

$$
\begin{aligned}
& (v) n N(n, 0,1,1,1)=\sum_{\substack{d \mid n \\
d \equiv 1}} \mu(d) F(n / d, 0,1,1,1)+\sum_{\substack{d \mid n \\
d \equiv 3}} \mu(d) F(n / d, 0,1,1,0) \text {, } \\
& n N(n, 0,1,1,0)=\sum_{\substack{d \mid n \\
d \equiv 1}} \mu(d) F(n / d, 0,1,1,0)+\sum_{\substack{d \mid n \\
d \equiv 3}} \mu(d) F(n / d, 0,1,1,1), \\
& \text { (vi) } n N(n, 0,0,0,0)=\sum_{\substack{d \mid n \\
d \text { odd }}} \mu(d) F(n / d, 0,0,0,0)-\sum_{\substack{d \mid n, \frac{n}{d} \text { even } \\
\text { odd }}} \mu(d) F(n / 2 d, 0,0) \\
& \text { (vii) } n N(n, 0,0,0,1)=\sum_{\substack{d \mid n \\
d \text { odd }}} \mu(d) F(n / d, 0,0,0,1)-\sum_{\substack{d \mid n, \frac{n}{d} \text { even } \\
d \text { odd }}} \mu(d) F(n / 2 d, 0,1) \text {, } \\
& \text { (viii) } n N(n, 0,1,0,0)=\sum_{\substack{d \mid n \\
d \equiv 1}} \mu(d) F(n / d, 0,1,0,0)-\sum_{\substack{d \mid n, \frac{n}{d} \text { even } \\
d \equiv 1}} \mu(d) F(n / 2 d, 1,0) \\
& +\sum_{\substack{d \mid n \\
d \equiv 3}} \mu(d) F(n / d, 0,1,0,1)-\sum_{\substack{d \mid n, \frac{n}{d} \text { even } \\
d=3}} \mu(d) F(n / 2 d, 1,1), \\
& n N(n, 0,1,0,1)=\sum_{\substack{d \mid n \\
d \equiv 1}} \mu(d) F(n / d, 0,1,0,1)-\sum_{\substack{d \mid n, \frac{n}{d} \text { even } \\
d \equiv=1}} \mu(d) F(n / 2 d, 1,1) \\
& +\sum_{\substack{d \mid n \\
d \equiv 3}} \mu(d) F(n / d, 0,1,0,0)-\sum_{\substack{d \mid n, \frac{n}{d} \text { even } \\
d \equiv 3}}^{d \equiv 1} \mu(d) F(n / 2 d, 1,0) .
\end{aligned}
$$


Proof. Suppose that

$$
N\left(n, t_{1}, t_{2}, t_{3}, t_{4}\right)=\left|\left\{f \in P(n): T_{i}(f)=t_{i}, i=1,2,3,4\right\}\right| .
$$

For different $t_{i} \in \mathbb{F}_{2}$, where $i=1,2,3,4$ we use Lemma 49 to compute the number $F\left(n, t_{1}, t_{2}, t_{3}, t_{4}\right)$.

(i) If $t_{1}=t_{2}=t_{3}=1$, and $t_{4}=0$, then we get

$$
\begin{aligned}
F(n, 1,1,1,0)= & \sum_{\substack{d \mid n \\
d \equiv 1}} \frac{n}{d} \cdot\left|\left\{f \in P(n / d): T_{i}(f)=1, i=1,2,3, T_{4}(f)=0\right\}\right| \\
& +\sum_{\substack{d \mid n \\
d \equiv 3}} \frac{n}{d} \cdot\left|\left\{f \in P(n / d): T_{1}(f)=T_{4}(f)=1, T_{2}(f)=T_{3}(f)=0\right\}\right| \\
& +\sum_{\substack{d \mid n \\
d \equiv 5}} \frac{n}{d} \cdot\left|\left\{f \in P(n / d): T_{i}(f)=1, i=1,2,3,4\right\}\right| \\
& +\sum_{\substack{d \mid n \\
d \equiv 7}} \frac{n}{d} \cdot\left|\left\{f \in P(n / d): T_{1}(f)=1, T_{i}(f)=0, i=2,3,4\right\}\right| \\
& =\sum_{\substack{d \mid n \\
d \equiv 1}} \frac{n}{d} N(n / d, 1,1,1,0)+\sum_{\substack{d \mid n \\
d \equiv 3}} \frac{n}{d} N(n / d, 1,0,0,1) \\
& +\sum_{\substack{d \mid n \\
d \equiv 5}} \frac{n}{d} N(n / d, 1,1,1,1)+\sum_{\substack{d \mid n \\
d \equiv 7}} \frac{n}{d} N(n / d, 1,0,0,0) .
\end{aligned}
$$

This means the four cases $(1,1,1,0),(1,0,0,1),(1,1,1,1)$ and $(1,0,0,0)$ are related to each other. Indeed,

$$
\begin{aligned}
F(n, 1,0,0,1) & =\sum_{\substack{d \mid n \\
d \equiv=1}} \frac{n}{d} N(n / d, 1,0,0,1)+\sum_{\substack{d \mid n \\
d=3}} \frac{n}{d} N(n / d, 1,1,1,1) \\
& +\sum_{\substack{d \mid n \\
d \equiv 5}} \frac{n}{d} N(n / d, 1,0,0,0)+\sum_{\substack{d \mid n \\
d \equiv 7}} \frac{n}{d} N(n / d, 1,1,1,0)
\end{aligned}
$$




$$
\begin{aligned}
F(n, 1,1,1,1)= & \sum_{\substack{d \mid n \\
d \equiv 1}} \frac{n}{d} N(n / d, 1,1,1,1)+\sum_{\substack{d \mid n \\
d \equiv 3}} \frac{n}{d} N(n / d, 1,0,0,0) \\
& +\sum_{\substack{d \mid n \\
d \equiv 5}} \frac{n}{d} N(n / d, 1,1,1,0)+\sum_{\substack{d \mid n \\
d \equiv 7}} \frac{n}{d} N(n / d, 1,0,0,1) \\
F(n, 1,0,0,0)= & \sum_{\substack{d \mid n \\
d \equiv 1}} \frac{n}{d} N(n / d, 1,0,0,0)+\sum_{\substack{d \mid n \\
d \equiv 3}} \frac{n}{d} N(n / d, 1,1,1,0) \\
& +\sum_{\substack{d \mid n \\
d \equiv 5}} \frac{n}{d} N(n / d, 1,0,0,1)+\sum_{\substack{d \mid n \\
d \equiv 7}} \frac{n}{d} N(n / d, 1,1,1,1)
\end{aligned}
$$

Then by our generalization of Möbius inversion formula given in Theorem 24 we have

$$
\begin{aligned}
& n N(n, 1,1,1,0)=\sum_{\substack{d \mid n \\
d \equiv 1}} \mu(d) F(n / d, 1,1,1,0)+\sum_{\substack{d \mid n \\
d \equiv 3}} \mu(d) F(n / d, 1,0,0,0) \\
& +\sum_{\substack{d \mid n \\
d \equiv 5}} \mu(d) F(n / d, 1,1,1,1)+\sum_{\substack{d \mid n \\
d \equiv 7}} \mu(d) F(n / d, 1,0,0,1) \\
& n N(n, 1,0,0,1)=\sum_{\substack{d \mid n \\
d \equiv 1}} \mu(d) F(n / d, 1,0,0,1)+\sum_{\substack{d \mid n \\
d \equiv 3}} \mu(d) F(n / d, 1,1,1,0) \\
& +\sum_{\substack{d \mid n \\
d \equiv 5}} \mu(d) F(n / d, 1,0,0,0)+\sum_{\substack{d \mid n \\
d \equiv 7}} \mu(d) F(n / d, 1,1,1,1) \\
& n N(n, 1,1,1,1)=\sum_{\substack{d \mid n \\
d \equiv 1}} \mu(d) F(n / d, 1,1,1,1)+\sum_{\substack{d \mid n \\
d \equiv 3}} \mu(d) F(n / d, 1,0,0,1) \\
& +\sum_{\substack{d \mid n \\
d=5}} \mu(d) F(n / d, 1,1,1,0)+\sum_{\substack{d \mid n \\
d \equiv 7}} \mu(d) F(n / d, 1,0,0,0) \\
& n N(n, 1,0,0,0)=\sum_{\substack{d \mid n \\
d \equiv 1}} \mu(d) F(n / d, 1,0,0,0)+\sum_{\substack{d \mid n \\
d \equiv 3}} \mu(d) F(n / d, 1,1,1,1) \\
& +\sum_{\substack{d \mid n \\
d \equiv 5}} \mu(d) F(n / d, 1,0,0,1)+\sum_{\substack{d \mid n \\
d \equiv 7}} \mu(d) F(n / d, 1,1,1,0) .
\end{aligned}
$$

This proves $(i)$. 
(ii) If we suppose that $t_{1}=t_{2}=t_{4}=0$ and $t_{3}=1$, then

$$
\begin{aligned}
F(n, 0,0,1,0) & =\sum_{\substack{d \mid n \\
d \equiv 1}} \frac{n}{d} N(n / d, 0,0,1,0)+\sum_{\substack{d \mid n \\
d \equiv 3}} \frac{n}{d} N(n / d, 0,0,1,0) \\
& +\sum_{\substack{d \mid n \\
d \equiv 5}} \frac{n}{d} N(n / d, 0,0,1,0)+\sum_{\substack{d \mid n \\
d \equiv 7}} \frac{n}{d} N(n / d, 0,0,1,0)
\end{aligned}
$$

Since in all the sums $d$ is odd, then we have

$$
F(n, 0,0,1,0)=\sum_{\substack{d \mid n \\ d \text { odd }}} \frac{n}{d} N(n / d, 0,0,1,0)
$$

By applying Möbius inversion, the number $N(n, 0,0,1,0)$ can be given as,

$$
n N(n, 0,0,1,0)=\sum_{\substack{d \mid n \\ d \text { odd }}} \mu(d) F(n / d, 0,0,1,0)
$$

(iii) Now let $t_{1}=t_{2}=0$ and $t_{3}=t_{4}=1$. Then

$$
\begin{aligned}
F(n, 0,0,1,1) & =\sum_{\substack{d \mid n \\
d \equiv 1}} \frac{n}{d} N(n / d, 0,0,1,1)+\sum_{\substack{d \mid n \\
d \equiv 3}} \frac{n}{d} N(n / d, 0,0,1,1) \\
& +\sum_{\substack{d \mid n \\
d \equiv 5}} \frac{n}{d} N(n / d, 0,0,1,1)+\sum_{\substack{d \mid n \\
d \equiv 7}} \frac{n}{d} N(n / d, 0,0,1,1) \\
& =\sum_{\substack{d \mid n \\
d \text { odd }}} \frac{n}{d} N(n / d, 0,0,1,1) .
\end{aligned}
$$

Then by Möbius inversion we have

$$
n N(n, 0,0,1,1)=\sum_{\substack{d \mid n \\ d \text { odd }}} \mu(d) F(n / d, 0,0,1,1)
$$


(iv) By letting $t_{1}=t_{2}=1$, and $t_{3}=t_{4}=0$ we have

$$
\begin{aligned}
F(n, 1,1,0,0) & =\sum_{\substack{d \mid n \\
d \equiv 1}} \frac{n}{d} N(n / d, 1,1,0,0)+\sum_{\substack{d \mid n \\
d \equiv 3}} \frac{n}{d} N(n / d, 1,0,1,1) \\
& +\sum_{\substack{d \mid n \\
d \equiv 5}} \frac{n}{d} N(n / d, 1,1,0,1)+\sum_{\substack{d \mid n \\
d \equiv 7}} \frac{n}{d} N(n / d, 1,0,1,0)
\end{aligned}
$$

This means the four cases $(1,1,0,0),(1,0,1,1),(1,1,0,1)$ and $(1,0,1,0)$ are related, and

$$
\begin{aligned}
F(n, 1,0,1,1)= & \sum_{\substack{d \mid n \\
d \equiv 1}} \frac{n}{d} N(n / d, 1,0,1,1)+\sum_{\substack{d \mid n \\
d \equiv 3}} \frac{n}{d} N(n / d, 1,1,0,1) \\
& +\sum_{\substack{d \mid n \\
d \equiv 5}} \frac{n}{d} N(n / d, 1,0,1,0)+\sum_{\substack{d \mid n \\
d \equiv 7}} \frac{n}{d} N(n / d, 1,1,0,0) \\
F(n, 1,1,0,1)= & \sum_{\substack{d \mid n \\
d \equiv 1}} \frac{n}{d} N(n / d, 1,1,0,1)+\sum_{\substack{d \mid n \\
d \equiv 3}} \frac{n}{d} N(n / d, 1,0,1,0) \\
+ & \sum_{\substack{d \mid n \\
d \equiv 5}} \frac{n}{d} N(n / d, 1,1,0,0)+\sum_{\substack{d \mid n \\
d \equiv 7}} \frac{n}{d} N(n / d, 1,0,1,1) \\
F(n, 1,0,1,0)= & \sum_{\substack{d \mid n \\
d \equiv 1}} \frac{n}{d} N(n / d, 1,0,1,0)+\sum_{\substack{d \mid n \\
d \equiv 3}} \frac{n}{d} N(n / d, 1,1,0,0) \\
+ & \sum_{\substack{d \mid n \\
d \equiv 5}} \frac{n}{d} N(n / d, 1,0,1,1)+\sum_{\substack{d \mid n \\
d \equiv 7}} \frac{n}{d} N(n / d, 1,1,0,1) .
\end{aligned}
$$

Hence by Theorem 24 we have

$$
\begin{aligned}
n N(n, 1,1,0,0) & =\sum_{\substack{d \mid n \\
d \equiv 1}} \mu(d) F(n / d, 1,1,0,0)+\sum_{\substack{d \mid n \\
d \equiv 3}} \mu(d) F(n / d, 1,0,1,0) \\
& +\sum_{\substack{d \mid n \\
d \equiv 5}} \mu(d) F(n / d, 1,1,0,1)+\sum_{\substack{d \mid n \\
d \equiv 7}} \mu(d) F(n / d, 1,0,1,1),
\end{aligned}
$$




$$
\begin{aligned}
n N(n, 1,0,1,1)= & \sum_{\substack{d \mid n \\
d \equiv 1}} \mu(d) F(n / d, 1,0,1,1)+\sum_{\substack{d \mid n \\
d \equiv 3}} \mu(d) F(n / d, 1,1,0,0) \\
& +\sum_{\substack{d \mid n \\
d \equiv 5}} \mu(d) F(n / d, 1,0,1,0)+\sum_{\substack{d \mid n \\
d \equiv 7}} \mu(d) F(n / d, 1,1,0,1), \\
n N(n, 1,1,0,1)= & \sum_{\substack{d \mid n \\
d \equiv 1}} \mu(d) F(n / d, 1,1,0,1)+\sum_{\substack{d \mid n \\
d \equiv 3}} \mu(d) F(n / d, 1,0,1,1) \\
+ & \sum_{\substack{d \mid n \\
d \equiv 5}} \mu(d) F(n / d, 1,1,0,0)+\sum_{\substack{d \mid n \\
d \equiv 7}} \mu(d) F(n / d, 1,0,1,0), \\
n N(n, 1,0,1,0)= & \sum_{\substack{d \mid n \\
d \equiv 1}} \mu(d) F(n / d, 1,0,1,0)+\sum_{\substack{d \mid n \\
d \equiv 3}} \mu(d) F(n / d, 1,1,0,1) \\
+ & \sum_{\substack{d \mid n \\
d \equiv 5}} \mu(d) F(n / d, 1,0,1,1)+\sum_{\substack{d \mid n \\
d \equiv 7}} \mu(d) F(n / d, 1,1,0,0) .
\end{aligned}
$$

(v) If $t_{1}=0$, and $t_{2}=t_{3}=t_{4}=1$, then

$$
\begin{aligned}
F(n, 0,1,1,1) & =\sum_{\substack{d \mid n \\
d \equiv 1}} \frac{n}{d} N(n / d, 0,1,1,1)+\sum_{\substack{d \mid n \\
d \equiv 3}} \frac{n}{d} N(n / d, 0,1,1,0) \\
& +\sum_{\substack{d \mid n \\
d \equiv 5}} \frac{n}{d} N(n / d, 0,1,1,1)+\sum_{\substack{d \mid n \\
d \equiv 7}} \frac{n}{d} N(n / d, 0,1,1,0) \\
& =\sum_{\substack{d \mid n \\
d \equiv 1}} \frac{n}{d} N(n / d, 0,1,1,1)+\sum_{\substack{d \mid n \\
d \equiv 5}} \frac{n}{d} N(n / d, 0,1,1,1) \\
& +\sum_{\substack{d \mid n \\
d \equiv 3}} \frac{n}{d} N(n / d, 0,1,1,0)+\sum_{\substack{d \mid n \\
d \equiv 7}} \frac{n}{d} N(n / d, 0,1,1,0) \\
& =\sum_{\substack{d \mid n \\
d \equiv(\bmod 4)}} \frac{n}{d} N(n / d, 0,1,1,1)+\sum_{\substack{d \mid n \\
d \equiv 3(\bmod 4)}} \frac{n}{d} N(n / d, 0,1,1,0) .
\end{aligned}
$$

This implies that the cases $(0,1,1,1)$ and $(0,1,1,0)$ are related to each other. In a 
similar way we have

$$
F(n, 0,1,1,0)=\sum_{\substack{d \mid n \\ d \equiv 1(\bmod 4)}} \frac{n}{d} N(n / d, 0,1,1,0)+\sum_{\substack{d \mid n \\ d \equiv 3(\bmod 4)}} \frac{n}{d} N(n / d, 0,1,1,1)
$$

Thus by Theorem 21 we have

$$
\begin{aligned}
n N(n, 0,1,1,1) & =\sum_{\substack{d \mid n \\
d \equiv 1(\bmod 4)}} \mu(d) F(n / d, 0,1,1,1)+\sum_{\substack{d \mid n \\
d \equiv 3(\bmod 4)}} \mu(d) F(n / d, 0,1,1,0), \\
n N(n, 0,1,1,0) & =\sum_{\substack{d \mid n \\
d \equiv 1(\bmod 4)}} \mu(d) F(n / d, 0,1,1,0)+\sum_{\substack{d \mid n \\
d \equiv 3(\bmod 4)}} \mu(d) F(n / d, 0,1,1,1) .
\end{aligned}
$$

(vi) Suppose that $t_{1}=t_{2}=t_{3}=t_{4}=0$. Then,

$$
\begin{aligned}
F(n, 0,0,0,0)= & \sum_{\substack{d \mid n \\
d \equiv 0}} \frac{n}{d} N(n / d)+\sum_{\substack{d \mid n \\
d \equiv 1}} \frac{n}{d} N(n / d, 0,0,0,0) \\
& +\sum_{\substack{d \mid n \\
d \equiv 2}} \frac{n}{d} N(n / d, 0,0)+\sum_{\substack{d \mid n \\
d \equiv 3}} \frac{n}{d} N(n / d, 0,0,0,0) \\
& +\sum_{\substack{d \mid n \\
d \equiv 4}} \frac{n}{d} N(n / d, 0)+\sum_{\substack{d \mid n \\
d \equiv 5}} \frac{n}{d} N(n / d, 0,0,0,0) \\
& +\sum_{\substack{d \mid n \\
d \equiv 6}} \frac{n}{d} N(n / d, 0,0)+\sum_{\substack{d \mid n \\
d \equiv 7}} \frac{n}{d} N(n / d, 0,0,0,0) \\
= & \sum_{\substack{d \mid n \\
d \text { odd }}} \frac{n}{d} N(n / d, 0,0,0,0)+\sum_{\substack{d \mid n \\
d \equiv 0}} \frac{n}{d} N(n / d)+\sum_{\substack{d \mid n \\
d \equiv 4}} \frac{n}{d} N(n / d, 0) \\
+ & \sum_{\substack{d \mid n \\
d \equiv 2}} \frac{n}{d} N(n / d, 0,0)+\sum_{\substack{d \mid n \\
d \equiv 6}} \frac{n}{d} N(n / d, 0,0) .
\end{aligned}
$$

Let us simplify the last four sums in $F(n, 0,0,0,0)$. If $d \equiv 0(\bmod 8)$, then $d=8 k=2(4 k)$, for some $k$. Therefore, $d / 2=4 k$. Let $d^{\prime}=\frac{d}{2}$, and $n^{\prime}=\frac{n}{2}$. So from 
$d=2(4 k)$ we have $d^{\prime}=4 k$, or $d^{\prime} \equiv 0(\bmod 4)$. Also $d \mid n$ implies $2 d^{\prime} \mid 2 n^{\prime}$, or $d^{\prime} \mid n^{\prime}$.

Therefore,

$$
\sum_{\substack{d \mid n \\ d \equiv 0}} \frac{n}{d} N(n / d)=\sum_{\substack{d^{\prime} \mid n^{\prime} \\ d^{\prime} \equiv 0(\bmod 4)}} \frac{n^{\prime}}{d^{\prime}} N\left(n^{\prime} / d^{\prime}\right)
$$

Similarly,

$$
\begin{aligned}
\sum_{\substack{d \mid n \\
d \equiv 4}} \frac{n}{d} N(n / d, 0) & =\sum_{\substack{d^{\prime} \mid n^{\prime} \\
d^{\prime}}} \frac{n^{\prime}(\bmod 4)}{d^{\prime}} N\left(n^{\prime} / d^{\prime}, 0\right), \\
\sum_{\substack{d \mid n \\
d \equiv 2}} \frac{n}{d} N(n / d, 0,0) & =\sum_{\substack{d^{\prime} \mid n^{\prime} \\
d^{\prime}}} \frac{n^{\prime}}{d^{\prime}(\bmod 4)} N\left(n^{\prime} / d^{\prime}, 0,0\right),
\end{aligned}
$$

and

$$
\sum_{\substack{d \mid n \\ d=6}} \frac{n}{d} N(n / d, 0,0)=\sum_{\substack{d^{\prime} \mid n^{\prime} \\ d^{\prime} \equiv 3(\bmod 4)}} \frac{n^{\prime}}{d^{\prime}} N\left(n^{\prime} / d^{\prime}, 0,0\right) .
$$

Then by Lemma 40 ,

$$
\sum_{\substack{d^{\prime} \mid n^{\prime} \\ d^{\prime} \equiv 0(\bmod 4)}} \frac{n^{\prime}}{d^{\prime}} N\left(n^{\prime} / d^{\prime}\right)+\sum_{\substack{d^{\prime} \mid n^{\prime} \\ d^{\prime} \equiv 2(\bmod 4)}} \frac{n^{\prime}}{d^{\prime}} N\left(n^{\prime} / d^{\prime}, 0\right)=\left[n^{\prime} \text { even }\right] 2^{\frac{n^{\prime}}{2}-1}
$$

Moreover,

$$
\sum_{\substack{d^{\prime} \mid n^{\prime} \\ d^{\prime} \equiv 1(\bmod 4)}} \frac{n^{\prime}}{d^{\prime}} N\left(n^{\prime} / d^{\prime}, 0,0\right)+\sum_{\substack{d^{\prime} \mid n^{\prime} \\ d^{\prime} \equiv 3(\bmod 4)}} \frac{n^{\prime}}{d^{\prime}} N\left(n^{\prime} / d^{\prime}, 0,0\right)=\sum_{\substack{d^{\prime} n^{\prime} \\ d^{\prime} \text { odd }}} \frac{n^{\prime}}{d^{\prime}} N\left(n^{\prime} / d^{\prime}, 0,0\right)
$$

Now by Theorem $43(i)$ we have

$$
\left[n^{\prime} \text { even }\right] 2^{\frac{n^{\prime}}{2}-1}+\sum_{\substack{d^{\prime} n^{\prime} \\ d^{\prime} \text { odd }}} \frac{n^{\prime}}{d^{\prime}} N\left(n^{\prime} / d^{\prime}, 0,0\right)=F\left(n^{\prime}, 0,0\right)=F(n / 2,0,0)
$$


Then the equation for $F(n, 0,0,0,0)$ changes to

$$
F(n, 0,0,0,0)=\sum_{\substack{d \mid n \\ d \text { odd }}} \frac{n}{d} N(n / d, 0,0,0,0)+F(n / 2,0,0)
$$

or

$$
F(n, 0,0,0,0)-F(n / 2,0,0)=\sum_{\substack{d \mid n \\ d \text { odd }}} \frac{n}{d} N(n / d, 0,0,0,0) .
$$

We have $n=2 n^{\prime}$. Thus $d \mid n$, and $d$ odd implies $d \mid n^{\prime}$. Therefore $n^{\prime}=d \lambda$ for some $\lambda$, or $\frac{n}{2}=d \lambda$. Thus $\frac{n}{d}=2 \lambda$. So $\frac{n}{d}$ is an even integer. Therefore by Theorem 20 and previous equation we have

$$
n N(n, 0,0,0,0)=\sum_{\begin{array}{c}
d \mid n \\
d \text { odd }
\end{array}} \mu(d) F(n / d, 0,0,0,0)-\sum_{\begin{array}{c}
d \mid n, \frac{n}{d} \text { even } \\
d \text { odd }
\end{array}} \mu(d) F(n / 2 d, 0,0) .
$$

(vii) Let $t_{1}=t_{2}=t_{3}=0$ and $t_{4}=1$, then

$$
\begin{aligned}
F(n, 0,0,0,1)= & \sum_{\substack{d \mid n \\
d \equiv=1}} \frac{n}{d} N(n / d, 0,0,0,1)+\sum_{\substack{d \mid n \\
d \equiv 2}} \frac{n}{d} N(n / d, 0,1) \\
& +\sum_{\substack{d \mid n \\
d \equiv 3}} \frac{n}{d} N(n / d, 0,0,0,1)+\sum_{\substack{d \mid n \\
d \equiv=4}} \frac{n}{d} N(n / d, 1) \\
& +\sum_{\substack{d \mid n \\
d \equiv 5}} \frac{n}{d} N(n / d, 0,0,0,1)+\sum_{\substack{d \mid n \\
d \equiv=6}} \frac{n}{d} N(n / d, 0,1) \\
& +\sum_{\substack{d \mid n \\
d \equiv 7}} \frac{n}{d} N(n / d, 0,0,0,1) \\
= & \sum_{\substack{d|n \\
d| n}} \frac{n}{d} N(n / d, 0,0,0,1)+\sum_{\substack{d \mid n \\
d=2}} \frac{n}{d} N(n / d, 0,1) \\
& +\sum_{\substack{d \mid n \\
d \equiv 4}} \frac{n}{d} N(n / d, 1)+\sum_{\substack{d \mid n \\
d \equiv 6}} \frac{n}{d} N(n / d, 0,1)
\end{aligned}
$$


To simplify these sums, first by Lemma 42 , we have

$$
\sum_{\substack{d \mid n \\ d \equiv 4}} \frac{n}{d} N(n / d, 1)=\sum_{\substack{d^{\prime} \mid n^{\prime} \\ d^{\prime} \equiv 2(\bmod 4)}} \frac{n^{\prime}}{d^{\prime}} N\left(n^{\prime} / d^{\prime}, 1\right)=\left[n^{\prime} \text { even }\right] 2^{\frac{n^{\prime}}{2}-1}
$$

where $d^{\prime}=\frac{d}{2}$, and $n^{\prime}=\frac{n}{2}$. Similar to the case $t_{1}=t_{2}=t_{3}=t_{4}=0$, we have

$$
\sum_{\substack{d \mid n \\ d \equiv 2}} \frac{n}{d} N(n / d, 0,1)=\sum_{\substack{d^{\prime} \mid n^{\prime} \\ d^{\prime} \equiv 1}} \frac{n^{\prime}}{d^{\prime}} N\left(n^{\prime} / d^{\prime}, 0,1\right)
$$

and

$$
\sum_{\substack{d \mid n \\ d \equiv 6}} \frac{n}{d} N(n / d, 0,1)=\sum_{\substack{d^{\prime} \mid n^{\prime} \\ d^{\prime} \equiv 3}} \frac{n^{\prime}}{d^{\prime}} N\left(n^{\prime} / d^{\prime}, 0,1\right)
$$

where in the new sums $a \equiv b$ denotes $a \equiv b(\bmod 4)$. Moreover, if we add them together, we have

$$
\sum_{\substack{d \mid n \\ d \equiv 2}} \frac{n}{d} N(n / d, 0,1)+\sum_{\substack{d \mid n \\ d \equiv 6}} \frac{n}{d} N(n / d, 0,1)=\sum_{\substack{d^{\prime} \mid n^{\prime} \\ d^{\prime} \text { odd }}} \frac{n^{\prime}}{d^{\prime}} N\left(n^{\prime} / d^{\prime}, 0,1\right)
$$

Then the last three sums in $F(n, 0,0,0,1)$ can be simplified as

$$
\left[n^{\prime} \text { even }\right] 2^{\frac{n^{\prime}}{2}-1}+\sum_{\substack{d^{\prime} \mid n^{\prime} \\ d^{\prime} \text { odd }}} \frac{n^{\prime}}{d^{\prime}} N\left(n^{\prime} / d^{\prime}, 0,1\right)
$$

which by Theorem $43(i i)$ simplifies to $F\left(n^{\prime}, 0,1\right)$, or $F(n / 2,0,1)$. Thus the number $F(n, 0,0,0,1)$ can be given as,

$$
F(n, 0,0,0,1)=\sum_{\substack{d \mid n \\ d \text { odd }}} \frac{n}{d} N(n / d, 0,0,0,1)+F(n / 2,0,1)
$$


which implies

$$
F(n, 0,0,0,1)-F(n / 2,0,1)=\sum_{\substack{d \mid n \\ d \text { odd }}} \frac{n}{d} N(n / d, 0,0,0,1)
$$

Then by Theorem 20 we have

$$
n N(n, 0,0,0,1)=\sum_{\substack{d \mid n \\ d \text { odd }}} \mu(d) F(n / d, 0,0,0,1)-\sum_{\substack{d \mid n, \frac{n}{d} \text { even } \\ d \text { odd }}} \mu(d) F(n / 2 d, 0,1)
$$

(viii) Now let $t_{1}=t_{3}=t_{4}=0$ and $t_{2}=1$. Then

$$
\begin{aligned}
F(n, 0,1,0,0) & =\sum_{\substack{d \mid n \\
d \equiv 1}} \frac{n}{d} N(n / d, 0,1,0,0)+\sum_{\substack{d \mid n \\
d \equiv 2}} \frac{n}{d} N(n / d, 1,0) \\
& +\sum_{\substack{d \mid n \\
d \equiv 3}} \frac{n}{d} N(n / d, 0,1,0,1)+\sum_{\substack{d \mid n \\
d \equiv 5}} \frac{n}{d} N(n / d, 0,1,0,0) \\
& +\sum_{\substack{d \mid n \\
d \equiv 6}} \frac{n}{d} N(n / d, 1,1)+\sum_{\substack{d \mid n \\
d \equiv 7}} \frac{n}{d} N(n / d, 0,1,0,1) .
\end{aligned}
$$

This means the two cases $(0,1,0,0)$ and $(0,1,0,1)$ are connected. Indeed,

$$
\begin{aligned}
F(n, 0,1,0,1) & =\sum_{\substack{d \mid n \\
d \equiv 1}} \frac{n}{d} N(n / d, 0,1,0,1)+\sum_{\substack{d \mid n \\
d \equiv 2}} \frac{n}{d} N(n / d, 1,1) \\
& +\sum_{\substack{d \mid n \\
d \equiv 3}} \frac{n}{d} N(n / d, 0,1,0,0)+\sum_{\substack{d \mid n \\
d \equiv 5}} \frac{n}{d} N(n / d, 0,1,0,1) \\
& +\sum_{\substack{d \mid n \\
d \equiv 6}} \frac{n}{d} N(n / d, 1,0)+\sum_{\substack{d \mid n \\
d \equiv 7}} \frac{n}{d} N(n / d, 0,1,0,0) .
\end{aligned}
$$


Let us simplify each part of $F(n, 0,1,0,0)$. We have

$$
\sum_{\substack{d \mid n \\ d \equiv 1}} \frac{n}{d} N(n / d, 0,1,0,0)+\sum_{\substack{d \mid n \\ d \equiv 5}} \frac{n}{d} N(n / d, 0,1,0,0)=\sum_{\substack{d \mid n \\ d \equiv 1(\bmod 4)}} \frac{n}{d} N(n / d, 0,1,0,0)
$$

Also,

$$
\sum_{\substack{d \mid n \\ d \equiv 3}} \frac{n}{d} N(n / d, 0,1,0,1)+\sum_{\substack{d \mid n \\ d \equiv 7}} \frac{n}{d} N(n / d, 0,1,0,1)=\sum_{\substack{d \mid n \\ d \equiv 3(\bmod 4)}} \frac{n}{d} N(n / d, 0,1,0,1)
$$

If $d \equiv 2(\bmod 8)$ and $d \equiv 6(\bmod 8)$, then $d$ is an even integer. The number $n$ is also even. If we replace $n$ by $n^{\prime}=n / 2$, and $d$ by $d^{\prime}=d / 2$, then

$$
\begin{aligned}
\sum_{\substack{d \mid n \\
d \equiv 2}} \frac{n}{d} N(n / d, 1,0)+\sum_{\substack{d \mid n \\
d \equiv 6}} \frac{n}{d} N(n / d, 1,1) & =\sum_{\substack{d^{\prime} n^{\prime} \\
d^{\prime} \equiv 1}} \frac{n^{\prime}}{d^{\prime}} N\left(n^{\prime} / d^{\prime}, 1,0\right) \\
& +\sum_{\substack{d^{\prime} \mid n^{\prime} \\
d^{\prime} \equiv 3}} \frac{n^{\prime}}{d^{\prime}} N\left(n^{\prime} / d^{\prime}, 1,1\right) .
\end{aligned}
$$

Then by Theorem $43($ iii $)$ for the new sums we have

$$
\sum_{\substack{d^{\prime}, n^{\prime} \\ d^{\prime} \equiv 1}} \frac{n^{\prime}}{d^{\prime}} N\left(n^{\prime} / d^{\prime}, 1,0\right)+\sum_{\substack{d^{\prime} / n^{\prime} \\ d^{\prime} \equiv 3}} \frac{n^{\prime}}{d^{\prime}} N\left(n^{\prime} / d^{\prime}, 1,1\right)=F\left(n^{\prime}, 1,0\right)=F(n / 2,1,0) .
$$

Therefore $F(n, 0,1,0,0)$ simplifies to

$$
\sum_{\substack{d \mid n \\ d \equiv 1}} \frac{n}{d} N(n / d, 0,1,0,0)+\sum_{\substack{d \mid n \\ d \equiv 3}} \frac{n}{d} N(n / d, 0,1,0,1)+F(n / 2,1,0)
$$

where in the new sums, $a \equiv b(\bmod 4)$ is represented by $a \equiv b$. 
To simplify $F(n, 0,1,0,1)$ we have

$$
\begin{aligned}
& \sum_{\substack{d \mid n \\
d \equiv 1}} \frac{n}{d} N(n / d, 0,1,0,1)+\sum_{\substack{d \mid n \\
d \equiv 5}} \frac{n}{d} N(n / d, 0,1,0,1)=\sum_{\substack{d \mid n \\
d \equiv 1(\bmod 4)}} \frac{n}{d} N(n / d, 0,1,0,1), \\
& \sum_{\substack{d \mid n \\
d \equiv 3}} \frac{n}{d} N(n / d, 0,1,0,0)+\sum_{\substack{d \mid n \\
d \equiv 7}} \frac{n}{d} N(n / d, 0,1,0,0)=\sum_{\substack{d \mid n \\
d \equiv 3(\bmod 4)}} \frac{n}{d} N(n / d, 0,1,0,0) .
\end{aligned}
$$

By Theorem 43(iv), we also have

$$
\begin{aligned}
\sum_{\substack{d \mid n \\
d \equiv 2}} \frac{n}{d} N(n / d, 1,1)+\sum_{\substack{d \mid n \\
d \equiv 6}} \frac{n}{d} N(n / d, 1,0) & =\sum_{\substack{d^{\prime} \mid n^{\prime} \\
d^{\prime} \equiv 1}} \frac{n^{\prime}}{d^{\prime}} N\left(n^{\prime} / d^{\prime}, 1,1\right) \\
& +\sum_{\substack{d^{\prime} \mid n^{\prime} \\
d^{\prime} \equiv 3}} \frac{n^{\prime}}{d^{\prime}} N\left(n^{\prime} / d^{\prime}, 1,0\right) \\
& =F\left(n^{\prime}, 1,1\right)=F(n / 2,1,1) .
\end{aligned}
$$

Thus $F(n, 0,1,0,1)$ simplifies to

$$
\sum_{\substack{d \mid n \\ d \equiv 1(\bmod 4)}} \frac{n}{d} N(n / d, 0,1,0,1)+\sum_{\substack{d \mid n \\ d \equiv 3(\bmod 4)}} \frac{n}{d} N(n / d, 0,1,0,0)+F(n / 2,1,1) .
$$

We then have

$$
\begin{aligned}
& F(n, 0,1,0,0)-F(n / 2,1,0)=\sum_{\substack{d \mid n \\
d \equiv 1}} \frac{n}{d} N(n / d, 0,1,0,0)+\sum_{\substack{d \mid n \\
d \equiv 3}} \frac{n}{d} N(n / d, 0,1,0,1), \\
& F(n, 0,1,0,1)-F(n / 2,1,1)=\sum_{\substack{d \mid n \\
d \equiv 1}} \frac{n}{d} N(n / d, 0,1,0,1)+\sum_{\substack{d \mid n \\
d \equiv 3}} \frac{n}{d} N(n / d, 0,1,0,0) .
\end{aligned}
$$


Therefore applying Möbius inversion, the number $N(n, 0,1,0,0)$ can be given as

$$
\begin{aligned}
n N(n, 0,1,0,0) & =\sum_{\substack{d \mid n \\
d \equiv 1}} \mu(d) F(n / d, 0,1,0,0)-\sum_{\substack{d \mid n, \frac{n}{d} \text { even } \\
d \equiv 1}} \mu(d) F(n / 2 d, 1,0) \\
& +\sum_{\substack{d \mid n \\
d \equiv 3}} \mu(d) F(n / d, 0,1,0,1)-\sum_{\substack{d \mid n, \frac{n}{d} \text { even } \\
d \equiv 3}} \mu(d) F(n / 2 d, 1,1) .
\end{aligned}
$$

In a similar way for $N(n, 0,1,0,1)$ we have

$$
\begin{aligned}
n N(n, 0,1,0,1) & =\sum_{\substack{d \mid n \\
d \equiv 1}} \mu(d) F(n / d, 0,1,0,1)-\sum_{\substack{d \mid n, \frac{n}{d} \text { even } \\
d \equiv 1}} \mu(d) F(n / 2 d, 1,1) \\
& +\sum_{\substack{d \mid n \\
d \equiv 3}} \mu(d) F(n / d, 0,1,0,0)-\sum_{\substack{d \mid n, \frac{n}{d} \text { even } \\
d \equiv 3}} \mu(d) F(n / 2 d, 1,0) .
\end{aligned}
$$

This ends the proof.

In Theorem 50 to find $N\left(n, t_{1}, t_{2}, t_{3}, t_{4}\right)$ for different values of $t_{i}$ which $i=1,2,3,4$ we need to compute $F\left(n, t_{1}, t_{2}, t_{3}, t_{4}\right)$. In the next chapter we study the number $F\left(n, t_{1}, t_{2}, t_{3}, t_{4}\right)$. Since the exact value of $F\left(n, t_{1}, t_{2}, t_{3}, t_{4}\right)$ was not possible to find, in Chapter 7 using the results in this chapter we give a good approximation for this number. 


\section{Chapter 6}

\section{An Attempt to Compute $F\left(n, t_{1}, t_{2}, t_{3}, t_{4}\right)$ Exactly}

In this chapter, to complete the computations of the number $N\left(n, t_{1}, t_{2}, t_{3}, t_{4}\right)$, we give a counting argument to find the value of $F\left(n, t_{1}, t_{2}, t_{3}, t_{4}\right)$, which is the number of elements $\gamma \in \mathbb{F}_{2^{n}}$ with $T_{i}(\gamma)=t_{i}$ and $i=1,2,3,4$.

In Section 6.1 we study the properties of the trace functions $T_{1}, T_{2}, T_{3}$ and $T_{4}$. In Section 6.2 we construct different cosets of $\mathbb{F}_{2^{l}}$ in $\mathbb{F}_{2^{n}}$, where $n=4 l$. Then in Section 6.3 we categorize different groups of cosets, and for each category we compute the traces of an element. Unfortunately it is quite challenging to find the exact formula for $F\left(n, t_{1}, t_{2}, t_{3}, t_{4}\right)$ in terms of $n, m$, and $l$. However, in Chapter 7 we are able to give a good approximation of $F\left(n, t_{1}, t_{2}, t_{3}, t_{4}\right)$ that leads to an estimate for the number $N\left(n, t_{1}, t_{2}, t_{3}, t_{4}\right)$, using the results from Chapter 5 . 


\subsection{Properties of Trace Functions}

We study different trace functions $T_{1}, T_{2}, T_{3}$, and $T_{4}$. For $i=1,2,3,4$, each $T_{i}$ is a function from $\mathbb{F}_{2^{n}}$ to $\mathbb{F}_{2}$. We find a relation between each of the traces $T_{2}, T_{3}$ and $T_{4}$ and the first trace $T_{1}$, and we also examine the linearity of each of the traces $T_{2}, T_{3}$ and $T_{4}$.

Since $n$ is even, let $n=2 m$. Therefore the finite field $\mathbb{F}_{2^{n}}$ has the subfield $\mathbb{F}_{2^{m}}$, where $\mathbb{F}_{2^{m}}=\left\{\beta \in \mathbb{F}_{2^{n}} \mid \beta^{2^{m}}+\beta=0\right\}$. If $m$ is an even number, we let $m=2 l$, where $l$ is a positive integer. Then $\mathbb{F}_{2^{n}}$ has the subfield $\mathbb{F}_{2^{l}}$. We write $T_{2^{m}}$ for the trace function from $\mathbb{F}_{2^{m}}$ to $\mathbb{F}_{2}$, and $T_{2^{l}}$ for the trace function from $\mathbb{F}_{2^{l}}$ to $\mathbb{F}_{2}$. The following proposition gives the connection of $T_{2}, T_{3}$ and $T_{4}$ with $T_{1}$.

Proposition 51. Let $n=2 m$, where $m$ is a positive integer. For $\beta \in \mathbb{F}_{2^{n}}$,

(i) $T_{2}(\beta)=\sum_{i=1}^{m-1} T_{1}\left(\beta \beta^{2^{i}}\right)+T_{2^{m}}\left(\beta \beta^{2^{m}}\right)$.

(ii) $T_{3}(\beta)=\sum_{i=1}^{n-2} \sum_{j=i+1}^{n-1} T_{1}\left(\beta \beta^{2^{i}} \beta^{2^{j}}\right)$.

(iii) If $n=4 l$, then

$$
\begin{aligned}
& T_{4}(\beta)=\sum_{i=1}^{m-2} \sum_{j=i+1}^{m} \sum_{k=j+1}^{m+1} T_{1}\left(\beta \beta^{2^{i}} \beta^{2^{j}} \beta^{2^{k}}\right)+\sum_{i=1}^{l-1} T_{2^{m}}\left(\beta \beta^{2^{i}} \beta^{2^{m}} \beta^{2^{m+i}}\right) \\
& +T_{2^{l}}\left(\beta \beta^{2^{l}} \beta^{2^{m}} \beta^{2^{m+l}}\right)+\sum_{k=2}^{l-1} \sum_{\substack { i=1 \\
\begin{subarray}{c}{j=k+2 \\
j>i{ i = 1 \\
\begin{subarray} { c } { j = k + 2 \\
j > i } }\end{subarray}}^{l-1} T_{1}\left(\beta \beta^{2^{i}} \beta^{2^{j}} \beta^{2^{m+k}}\right) \\
& +\sum_{k=2}^{l-2} \sum_{i=k+1}^{l-1} T_{1}\left(\beta \beta^{2^{i}} \beta^{2^{m}} \beta^{2^{m+k}}\right)+\sum_{k=2}^{l-1} T_{1}\left(\beta \beta^{2^{l}} \beta^{2^{m}} \beta^{2^{m+k}}\right) .
\end{aligned}
$$




$$
\begin{aligned}
& \text { If } n=4 l+2 \text {, then } \\
& T_{4}(\beta)=\sum_{i=1}^{m-2} \sum_{j=i+1}^{m} \sum_{k=j+1}^{m+1} T_{1}\left(\beta \beta^{2^{i}} \beta^{2^{j}} \beta^{2^{k}}\right)+\sum_{i=1}^{l} T_{2^{m}}\left(\beta \beta^{2^{i}} \beta^{2^{m}} \beta^{2^{m+i}}\right) \\
& +\sum_{k=2}^{l} \sum_{i=1}^{l} \sum_{\substack{i=k+2 \\
j>i}}^{m-1} T_{1}\left(\beta \beta^{2^{i}} \beta^{2^{j}} \beta^{2^{m+k}}\right)+\sum_{k=2}^{l-2} \sum_{i=k+1}^{l-1} T_{1}\left(\beta \beta^{2^{i}} \beta^{2^{m}} \beta^{2^{m+k}}\right) \\
& +\sum_{k=2}^{l-1} T_{1}\left(\beta \beta^{2^{l}} \beta^{2^{m}} \beta^{2^{m+k}}\right) \text {. }
\end{aligned}
$$

The proofs of $(i)$ and $(i i)$ are given in [24].

For $\beta \in \mathbb{F}_{2^{n}}$ by the definition of $T_{4}$ we have

$$
T_{4}(\beta)=\sum_{i_{1}} \sum_{i_{2}} \sum_{i_{3}} \sum_{i_{4}} \beta^{2^{i_{1}}} \beta^{2^{i_{2}}} \beta^{2^{i_{3}}} \beta^{2^{i_{4}}}
$$

We observe that the $T_{4}(\beta)$ formulas for $n=4 l$ and $n=4 l+2$ were quite challenging to find. It required some skillful computation with Maple to give the main contribution of $T_{4}(\beta)$ which is the first triple sum. From there by subtracting the main part from $T_{4}(\beta)$, and repeating this bootstrapping, we found the complete result. We checked the formulas for degree of extension $n$ up to 50 and they match perfectly.

In Proposition 51 the trace function $T_{4}$ is given for $n=4 l$, and $n=4 l+2$. For the rest of this chapter, we assume that $n=4 l$. Similar results can be obtained when $n=4 l+2$. Consider the mapping $\psi: \mathbb{F}_{2^{n}} \rightarrow \mathbb{F}_{2^{l}}$, such that for any $\gamma \in \mathbb{F}_{2^{n}}$ we have

$$
\psi(\gamma)=\gamma+\gamma^{2^{l}}+\gamma^{2^{2 l}}+\gamma^{2^{3 l}}=\hat{\gamma}
$$

For any $\gamma \in \mathbb{F}_{2^{3 l}}$ we have $\gamma=\gamma^{2^{3 l}}$. Therefore $\gamma=\gamma^{2^{l}}=\gamma^{2^{2 l}}=\gamma^{2^{3 l}}$ which means

$$
\operatorname{Ker}(\psi)=\left\{\gamma \in \mathbb{F}_{2^{n}} \mid \psi(\gamma)=0\right\}=\mathbb{F}_{2^{3 l}}
$$


and $\psi$ is a surjection. Therefore we have the following result.

Proposition 52. $\mathbb{F}_{2^{l}}$ and $\mathbb{F}_{2^{n}} / \mathbb{F}_{2^{3 l}}$ are isomorphic vector spaces.

Proposition 53. For any $\gamma \in \mathbb{F}_{2^{n}}, T_{1}(\gamma)=T_{2^{\iota}}(\hat{\gamma})$.

Proof.

$$
\begin{aligned}
T_{1}(\gamma) & =\sum_{i=0}^{n-1} \gamma^{2^{i}}=\sum_{i=0}^{l-1}\left(\gamma^{2^{i}}+\gamma^{2^{i+l}}+\gamma^{2^{i+2 l}}+\gamma^{2^{i+3 l}}\right) \\
& =\sum_{i=0}^{l-1}\left(\gamma+\gamma^{2^{l}}+\gamma^{2^{2 l}}+\gamma^{2^{3 l}}\right)^{2^{i}}=\sum_{i=0}^{l-1}(\hat{\gamma})^{2^{i}}=T_{2^{l}}(\hat{\gamma})
\end{aligned}
$$

For a given $\beta \in \mathbb{F}_{2^{l}}$ the following proposition gives the values of each of the traces $T_{i}$, for $1 \leq i \leq 4$.

Proposition 54. For any $\beta \in \mathbb{F}_{2^{l}}$ we have

(i) $T_{1}(\beta)=0$.

(ii) $T_{2}(\beta)=0$.

(iii) $T_{3}(\beta)=0$.

(iv) $T_{4}(\beta)=T_{2^{l}}(\beta)$.

Proof. Let $\beta \in \mathbb{F}_{2^{l}}$.

(i) Using Proposition 53 we have

$$
T_{1}(\beta)=T_{2^{l}}(\hat{\beta})=T_{2^{l}}\left(\beta+\beta^{2^{l}}+\beta^{2^{2 l}}+\beta^{2^{3 l}}\right) .
$$


Since $\beta \in \mathbb{F}_{q^{l}}$ then $\beta^{2^{l}}=\beta^{2^{2 l}}=\beta^{2^{3 l}}=\beta$, and therefore

$$
T_{1}(\beta)=T_{2^{l}}(\beta+\beta+\beta+\beta)=T_{2^{l}}(0)=0 .
$$

(ii) Clearly $\beta \beta^{2^{i}} \in \mathbb{F}_{2^{l}}$. Therefore by the first part $T_{1}\left(\beta \beta^{2^{i}}\right)=0$, which implies that $\sum_{i=1}^{m-1} T_{1}\left(\beta \beta^{2^{i}}\right)=0$. Moreover,

$$
T_{2^{m}}\left(\beta \beta^{2^{m}}\right)=T_{2^{m}}\left(\beta \beta^{2^{2 l}}\right)=T_{2^{m}}(\beta \beta)=T_{2^{m}}\left(\beta^{2}\right)=T_{2^{m}}(\beta)=0 .
$$

Then by Proposition 51 (i) we have

$$
T_{2}(\beta)=\sum_{i=1}^{m-1} T_{1}\left(\beta \beta^{2^{i}}\right)+T_{2^{m}}\left(\beta \beta^{2^{m}}\right)=0+0=0
$$

(iii) Since $\beta \in \mathbb{F}_{2^{l}}$, we have $\beta \beta^{2^{i}} \beta^{2^{j}} \in \mathbb{F}_{2^{l}}$, and $T_{1}\left(\beta \beta^{2^{i}} \beta^{2^{j}}\right)=0$, for all integers $i$ and $j$. Then the result follows by Proposition 51 (ii).

(iv) Similarly all the sums in $T_{4}$ given in Proposition 51 (iii) which are defined by $T_{1}$ and $T_{2^{l}}$ are zeros. Then

$$
T_{4}(\beta)=T_{2^{l}}\left(\beta \beta^{l} \beta^{m} \beta^{m+l}\right)=T_{2^{l}}\left(\beta \beta^{l} \beta^{2 l} \beta^{3 l}\right)=T_{2^{l}}\left(\beta^{4}\right)=T_{2^{l}}(\beta) .
$$

If $\beta$ is from $\mathbb{F}_{2^{l}}$ then it is important to examine the linearity of the traces $T_{2}, T_{3}$ and $T_{4}$. As we will show, the linearity of $T_{4}$ can be given using cyclotomic cosets. For the nonnegative integer $k=0,1, \ldots, \frac{l}{2}-1$ let $\left[1+2^{k}\right]$ be the cyclotomic coset defined as

$$
\left[1+2^{k}\right]=\left[\left(1+2^{k}\right)_{1},\left(1+2^{k}\right)_{2}, \ldots,\left(1+2^{k}\right)_{l}\right]
$$


where for each $k=0,1, \ldots, \frac{l}{2}-1$ we have

$$
\left(1+2^{k}\right)_{i} \equiv\left(1+2^{k}\right) 2^{i-1} \quad\left(\bmod 2^{l}-1\right)
$$

where $1 \leq i \leq l$. So for $k=0,1, \ldots, \frac{l}{2}-1$ each $\left[1+2^{k}\right]$ has length $l$. For $k=\frac{l}{2}$ which corresponds to the last coset $\left[\frac{l}{2}\right]$ the length is $\frac{l}{2}$. Therefore

$$
\left[\frac{l}{2}\right]=\left[\left(1+2^{\frac{l}{2}}\right)_{1},\left(1+2^{\frac{l}{2}}\right)_{2}, \ldots,\left(1+2^{\frac{l}{2}}\right) \frac{l}{2}\right]
$$

with the same definition for $\left(1+2^{\frac{l}{2}}\right)_{i}$ as in Equation(6.1). Let $n=4 l$ and $l$ be an even integer. For the nonnegative integer $k$ we use $\hat{\gamma}$ to define

$$
\delta_{1+2^{k}}= \begin{cases}\hat{\gamma} \hat{\gamma}^{2^{l-1}}+\hat{\gamma}\left(\sum_{i=1}^{l-2} \sum_{j=i+1}^{l-1} \hat{\gamma}^{2^{i}} \hat{\gamma}^{2^{j}}+\sum_{i=1}^{l-1} C_{2}\left(\hat{\gamma}^{2^{i}}\right)\right) & \\ \quad+\hat{\gamma}^{2^{l-1}}\left(\sum_{i=1}^{l-2} \hat{\gamma}^{i^{2}}\right)+C_{2}\left(\hat{\gamma}^{2^{l-1}}\right)+C_{3}(\hat{\gamma}) & \text { if } k=0, \\ \hat{\gamma}+\hat{\gamma}^{2}+\hat{\gamma} \hat{\gamma}^{2} & \text { if } k=1, \text { and } n=8, \\ \hat{\gamma}+\hat{\gamma} \hat{\gamma}^{2} & \text { if } k=1, \text { and } n>8, \\ \hat{\gamma} \hat{\gamma}^{2^{k}} & \text { if } k>1 .\end{cases}
$$

Also for $i=1,2, \ldots, l-1$ let us define

$$
C_{2}\left(\hat{\gamma}^{2^{i}}\right)=\sum_{j<k \in S} \gamma^{j} \gamma^{k}, \quad C_{3}(\hat{\gamma})=\sum_{i<j<k \in S^{\prime}} \gamma^{i} \gamma^{j} \gamma^{k}
$$

where $S=\left\{2^{i}, 2^{i+l}, 2^{i+2 l}, 2^{i+3 l}\right\}$ and $S^{\prime}=\left\{1,2^{l}, 2^{2 l}, 2^{3 l}\right\}$. If $n=4 l$ and $l$ is odd then 
$\delta_{1+2^{k}}$ are defined in a similar but simpler way

$$
\delta_{1+2^{k}}= \begin{cases}\hat{\gamma} \hat{\gamma}^{2-1}+\hat{\gamma}\left(\sum_{i=1}^{l-2} \sum_{j=i+1}^{l-1} \hat{\gamma}^{2^{2}} \hat{\gamma}^{2^{j}}+\sum_{i=1}^{l-1} C_{2}\left(\hat{\gamma}^{2^{i}}\right)\right) & \\ \quad+\hat{\gamma}^{2^{l-1}}\left(\sum_{i=1}^{l-2} \hat{\gamma}^{2^{i}}\right)+C_{2}\left(\hat{\gamma}^{2^{l-1}}\right)+C_{3}(\hat{\gamma}) & \text { if } k=0 \\ \hat{\gamma}+\hat{\gamma} \hat{\gamma}^{2} & \text { if } k=1 \\ \hat{\gamma} \hat{\gamma}^{2^{k}} & \text { if } k>1 .\end{cases}
$$

Let $n=4 l$ and $\gamma \in \mathbb{F}_{2^{n}}$. By assuming $\beta \in \mathbb{F}_{2^{l}}$ we expand $T_{i}(\beta+\gamma)$, where $i=2,3,4$. In the expansion we find the terms $T_{i}(\beta)$ and $T_{i}(\gamma)$ and some other terms. For $i=2,3$ the rest of the terms are defined by $\beta, \hat{\gamma}$ and trace function $T_{2^{l}}$.

For the case $T_{4}(\beta+\gamma)$ we examined the rest of the terms in this expansion. We noticed that the last part of $T_{4}(\beta+\gamma)$ depends on $l$ being even, or odd. In both cases, $T_{4}(\beta+\gamma)$ has terms which are powers of $\beta$, where the exponents are from cyclotomic cosets $\left(1+2^{k}\right)$ defined previously. For different powers of $\beta$, the coefficients are $\delta_{1+2^{k}}$, which are functions of $\hat{\gamma}$ and are defined earlier.

From experimental results for $T_{i}(\beta+\gamma)$, where $i=2,3,4$ and $n=4,8, \ldots, 48$, we obtain the following equations about the linearity of $T_{2}, T_{3}$ and $T_{4}$, when $\beta \in \mathbb{F}_{2^{l}}$, $\gamma \in \mathbb{F}_{2^{n}}$ and $\hat{\gamma}=\gamma+\gamma^{2^{l}}+\gamma^{2^{2 l}}+\gamma^{2^{3 l}}$ :

$$
\begin{aligned}
& T_{2}(\beta+\gamma)=T_{2}(\beta)+T_{2}(\gamma)+T_{2^{l}}(\beta \hat{\gamma}) \\
& T_{3}(\beta+\gamma)=T_{2}(\beta)+T_{3}(\gamma)+T_{2^{l}}\left(\beta^{2} \hat{\gamma}+\beta \hat{\gamma}^{2}\right)+T_{2^{l}}\left(\beta \hat{\gamma} T_{2^{l}}(\hat{\gamma})\right)
\end{aligned}
$$

For the trace $T_{4}$, if $n=4 l$ where $l$ is an even integer, then

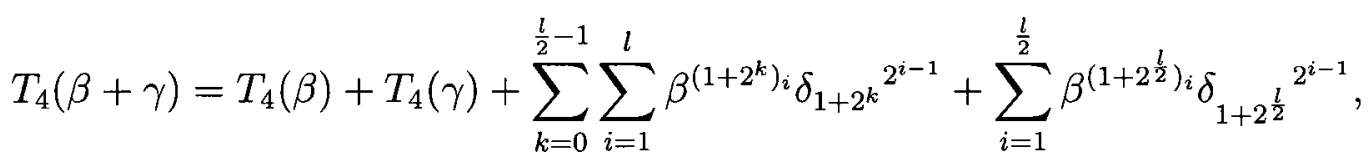

where the exponents of $\beta$ are from the cyclotomic coset $\left[1+2^{k}\right]$, and the coefficients 
of $\beta$ or $\delta_{1+2^{k}}$ are defined as before. If $n=4 l$ and $l$ is odd, then

$$
T_{4}(\beta+\gamma)=T_{4}(\beta)+T_{4}(\gamma)+\sum_{k=0}^{\frac{l-1}{2}} \sum_{i=1}^{l}{\beta^{\left(1+2^{k}\right)_{i}}}_{\delta_{1+2^{k}}}^{2^{i-1}}
$$

Indeed, $T_{4}(\gamma+\beta)$ can be given in a simpler way. If $n=4 l$ where $l=2 l^{\prime}+1$, then for $\beta \in \mathbb{F}_{2^{l}}$ and $\gamma \in \mathbb{F}_{2^{n}}$ we have

$$
T_{4}(\beta+\gamma)=T_{4}(\beta)+T_{4}(\gamma)+T_{2^{l}}\left(\sum_{k=0}^{l^{\prime}} \beta^{1+2^{k}} \delta_{1+2^{k}}\right)
$$

and if $l=2 l^{\prime}$, then $T_{4}$ can be given in terms of $T_{2^{l}}$ and $T_{2^{l^{\prime}}}$ as,

$$
T_{4}(\beta+\gamma)=T_{4}(\beta)+T_{4}(\gamma)+T_{2^{l}}\left(\sum_{k=0}^{l^{\prime}-1} \beta^{1+2^{k}} \delta_{1+2^{k}}\right)+T_{2^{l^{\prime}}}\left(\beta^{1+2^{i^{\prime}}} \delta_{1+2^{l^{\prime}}}\right)
$$

where the coefficients $\delta_{1+2^{l^{\prime}}}$ and $\delta_{1+2^{k}}, 0 \leq k \leq l^{\prime}-1$ are defined as before.

\subsection{Cosets of $\mathbb{F}_{2^{l}}$ in $\mathbb{F}_{2^{n}}$}

Suppose that $n=4 l$, and $\gamma \in \mathbb{F}_{2^{n}}$. In this section we examine different cosets $\gamma+\mathbb{F}_{2^{l}}$ which later we use to find the number $F\left(n, t_{1}, t_{2}, t_{3}, t_{4}\right)$, that is, the number of elements $\gamma \in \mathbb{F}_{2^{n}}$ with $T_{i}(\gamma)=t_{i} \in \mathbb{F}_{2}$, and $1, \ldots, 4$.

For $\alpha \in \mathbb{F}_{2^{l}}$ the linear functional $L_{\alpha}: \mathbb{F}_{2^{l}} \rightarrow \mathbb{F}_{2}$ is defined by the trace function $T_{2^{l}}$ as $L_{\alpha}(\beta)=T_{2^{l}}(\alpha \beta)$, for all $\beta \in \mathbb{F}_{2^{l}}$. In the special case $\alpha=0$ we have $L_{\alpha}=L_{0}$, and $L_{0}(\beta)=T_{2^{l}}(0 \beta)=T_{2^{l}}(0)=0$, for all $\beta \in \mathbb{F}_{2^{l}}$. Thus $\operatorname{Ker}\left(L_{0}\right)=\mathbb{F}_{2^{l}}$.

If we let $\alpha=1$, then $L_{\alpha}=L_{1}$ and $L_{1}(\beta)=T_{2^{l}}(\beta)$. So

$$
\operatorname{Ker}\left(L_{1}\right)=\left\{\beta \in \mathbb{F}_{2^{l}} \mid T_{2^{l}}(\beta)=0\right\}
$$


For any $\gamma \in \mathbb{F}_{2^{n}}$ we define

$$
W_{i}(\gamma)=\left\{\beta \in \mathbb{F}_{2^{l}} \mid T_{i}(\beta+\gamma)=T_{i}(\beta)+T_{i}(\gamma)\right\}, \quad 1 \leq i \leq 4
$$

Since $T_{1}$ is a linear mapping, then we have $W_{1}(\gamma)=\mathbb{F}_{2^{l}}$, for all $\gamma \in \mathbb{F}_{2^{n}}$. Suppose that $\phi_{2}$ represents the last part of $T_{2}$ given in the Equation (6.4), that is, $\phi_{2}(\beta)=T_{2^{l}}(\beta \hat{\gamma})$. Then from the definition of $W_{2}(\gamma)$ we have $W_{2}(\gamma)=\operatorname{Ker}\left(\phi_{2}\right)$. Clearly from the definitions of linear functional and $\phi_{2}$ we have that $\phi_{2}=L_{\hat{\gamma}}$. Therefore

$$
W_{2}(\gamma)=\operatorname{Ker}\left(\phi_{2}\right)=\operatorname{Ker}\left(L_{\hat{\gamma}}\right)
$$

Similarly, let $\phi_{3}(\beta)=T_{2^{l}}\left(\beta^{2} \hat{\gamma}+\beta \hat{\gamma}^{2}\right)+T_{2^{l}}\left(\beta \hat{\gamma} T_{2^{l}}(\hat{\gamma})\right)$. Then $W_{3}(\gamma)=\operatorname{Ker}\left(\phi_{3}\right)$.

Finally, let us define

$$
\phi_{4}(\beta)= \begin{cases}T_{2^{l}}\left(\sum_{k=0}^{l^{\prime}-1} \beta^{1+2^{k}} \delta_{1+2^{k}}\right)+T_{2^{l^{\prime}}}\left(\beta^{1+2^{l^{\prime}}} \delta_{1+2^{l^{\prime}}}\right) & \text { if } l=2 l^{\prime}, \\ T_{2^{l}}\left(\sum_{k=0}^{l^{\prime}} \beta^{1+2^{k}} \delta_{1+2^{k}}\right) & \text { if } l=2 l^{\prime}+1\end{cases}
$$

where the coefficients $\delta_{1+2^{k}}$ are functions of $\hat{\gamma}$ defined earlier in Section 6.1. Then for each $\gamma \in \mathbb{F}_{2^{n}}$ we have $W_{4}(\gamma)=\operatorname{Ker}\left(\phi_{4}\right)$. For $i=2,3,4$ the functions $\phi_{i}$ are defined by $T_{2^{l}}$ and are linear. Moreover the following property of $W_{2}(\gamma)$ and $W_{3}(\gamma)$ is proved in [24], and it is also true for $W_{4}(\gamma)$.

Proposition 55. For $\gamma \in \mathbb{F}_{2^{n}}$ and $i=2,3,4$, either $W_{i}(\gamma)=\mathbb{F}_{2^{l}}$ or $W_{i}(\gamma)$ is a hyperplane in $\mathbb{F}_{2^{l}}$.

Let us define

$$
W_{0}=\operatorname{Ker}\left(T_{2^{l}}\right)=\left\{\beta \in \mathbb{F}_{2^{l}} \mid T_{2^{l}}(\beta)=0\right\}
$$

Therefore $W_{0}$ is a proper subset of $\mathbb{F}_{2^{l}}$, and clearly $W_{0}=\operatorname{Ker}\left(L_{1}\right)$. 
In the rest of this section we present the necessary and sufficient conditions on $\gamma \in \mathbb{F}_{2^{n}}$ such that $W_{i}(\gamma)=\mathbb{F}_{2^{l}}, W_{i}(\gamma)=W_{0}$ or $W_{i}(\gamma) \neq W_{0}$, where $i=2,3,4$. The following proposition gives the condition to have $W_{2}(\gamma)=\mathbb{F}_{2^{l}}$.

Proposition 56. For any $\gamma \in \mathbb{F}_{2^{n}}$ we have $W_{2}(\gamma)=\mathbb{F}_{2^{l}}$ if and only if $\hat{\gamma}=0$. Otherwise $W_{2}(\gamma)$ is a hyperplane of $\mathbb{F}_{2^{l}}$.

Proof. Let $\gamma \in \mathbb{F}_{2^{n}}$ be such that $\hat{\gamma}=0$. Then

$$
\phi_{2}(\beta)=T_{2^{l}}(\beta \hat{\gamma})=T_{2^{l}}(0)=0
$$

for all $\beta \in \mathbb{F}_{2^{l}}$. So $T_{2}(\beta+\gamma)=T_{2}(\beta)+T_{2}(\gamma)$, for all $\beta \in \mathbb{F}_{2^{l}}$, or $W_{2}(\gamma)=\mathbb{F}_{2^{l}}$.

Now let $W_{2}(\gamma)=\mathbb{F}_{2^{l}}$. We show that $\hat{\gamma}=0$. Since

$$
\mathbb{F}_{2^{l}}=W_{2}(\gamma)=\operatorname{Ker}\left(\phi_{2}\right)=\operatorname{Ker}\left(L_{\hat{\gamma}}\right)=\left\{\beta \in \mathbb{F}_{2^{l}} \mid T_{2^{l}}(\beta \hat{\gamma})=0\right\}
$$

we have $T(\beta \hat{\gamma})=0$, for all $\beta \in \mathbb{F}_{2^{l}}$. This implies $\hat{\gamma}=0$.

The condition $\hat{\gamma}=0$ is a necessary and sufficient condition to have $W_{2}(\gamma)=\mathbb{F}_{2^{l}}$. Hence, for $\hat{\gamma} \neq 0$ by Proposition 55 the set $W_{2}(\gamma)$ is a hyperplane of $\mathbb{F}_{2^{l}}$.

The following proposition gives the necessary and sufficient conditions to have $W_{2}(\gamma)=W_{0}$

Proposition 57. Let $\gamma \in \mathbb{F}_{2^{n}}$. Then $W_{2}(\gamma)=W_{0}$ if and only if $\hat{\gamma}=1$.

Proof. For a given $\gamma \in \mathbb{F}_{2^{n}}$ we have

$$
W_{2}(\gamma)=\operatorname{Ker}\left(\phi_{2}\right)=\operatorname{Ker}\left(L_{\hat{\gamma}}\right)=\left\{\beta \in \mathbb{F}_{2^{l}} \mid T_{2^{l}}(\beta \hat{\gamma})=0\right\}
$$

If $\hat{\gamma}=1$ then clearly $W_{2}(\gamma)=\left\{\beta \in \mathbb{F}_{2^{l}} \mid T_{2^{l}}(\beta)=0\right\}=W_{0}$. 
Reciprocally, let $W_{2}(\gamma)=W_{0}$. Then we show that $\hat{\gamma}=1$. Since $W_{2}(\gamma)=\operatorname{Ker}\left(L_{\hat{\gamma}}\right)$ and $W_{0}=\operatorname{Ker}\left(L_{1}\right)$, then $W_{2}(\gamma)=W_{0}$ which implies that $\operatorname{Ker}\left(L_{\hat{\gamma}}\right)=\operatorname{Ker}\left(L_{1}\right)$, or $L_{\hat{\gamma}}=L_{1}$. Therefore we have $\hat{\gamma}=1$.

The following proposition gives $T_{3}$ in terms of a linear functional.

Proposition 58. For any $\gamma \in \mathbb{F}_{2^{n}}$,

(i) If $T_{2^{l}}(\hat{\gamma})=0$, then $\phi_{3}=L_{\hat{\gamma}^{2}+\hat{\gamma}^{l-1}}$.

(ii) If $T_{2^{l}}(\hat{\gamma})=1$, then $\phi_{3}=L_{\hat{\gamma}+\hat{\gamma}^{2}+\hat{\gamma}^{2 l-1}}$.

Proof. Let $\gamma \in \mathbb{F}_{2^{n}}$. If $T_{2^{l}}(\hat{\gamma})=0$ then $\phi_{3}(\beta)$ simplifies to

$$
\phi_{3}(\beta)=T_{2^{l}}\left(\beta^{2} \hat{\gamma}+\beta \hat{\gamma}^{2}\right)=T_{2^{l}}\left(\beta^{2} \hat{\gamma}\right)+T_{2^{l}}\left(\beta \hat{\gamma}^{2}\right)
$$

Since,

$$
\begin{aligned}
T_{2^{l}}\left(\beta^{2} \hat{\gamma}\right) & =\left(\beta^{2} \hat{\gamma}\right)+\left(\beta^{2} \hat{\gamma}\right)^{2}+\left(\beta^{2} \hat{\gamma}\right)^{2^{2}}+\cdots+\left(\beta^{2} \hat{\gamma}\right)^{2^{l-1}} \\
& =\beta^{2} \hat{\gamma}+\beta^{2^{2}} \hat{\gamma}^{2}+\beta^{2^{3}} \hat{\gamma}^{2^{2}}+\cdots+\beta^{2^{l}} \hat{\gamma}^{l^{l-1}} \\
& =\beta^{2} \hat{\gamma}+\beta^{2^{2}} \hat{\gamma}^{2}+\beta^{2^{3}} \hat{\gamma}^{2^{2}}+\cdots+\beta \hat{\gamma}^{2^{l-1}} \\
& =T_{2^{l}}\left(\beta \hat{\gamma}^{2^{l-1}}\right)
\end{aligned}
$$

then $\phi_{3}(\beta)$ changes to

$$
\phi_{3}(\beta)=T_{2^{l}}\left(\beta \hat{\gamma}^{2^{l-1}}\right)+T_{2^{l}}\left(\beta \hat{\gamma}^{2}\right)=T_{2^{l}}\left(\beta\left(\hat{\gamma}^{2}+\hat{\gamma}^{2^{l-1}}\right)\right)=L_{\hat{\gamma}^{2}+\hat{\gamma}^{l^{-1}}}(\beta),
$$

or $\phi_{3}=L_{\hat{\gamma}^{2}+\hat{\gamma}^{2-1}}$. 
If $T_{2^{l}}(\hat{\gamma}) \neq 0$, then $T_{2^{l}}(\hat{\gamma})=1$, so from the definition of $\phi_{3}$ we have

$$
\begin{aligned}
\phi_{3}(\beta) & =T_{2^{l}}\left(\beta^{2} \hat{\gamma}+\beta \hat{\gamma}^{2}\right)+T_{2^{l}}(\beta \hat{\gamma}) \\
& =T_{2^{l}}\left(\beta^{2} \hat{\gamma}\right)+T_{2^{l}}\left(\beta \hat{\gamma}^{2}\right)+T_{2^{l}}(\beta \hat{\gamma}) \\
& =T_{2^{l}}\left(\beta \hat{\gamma}^{2^{l-1}}\right)+T_{2^{l}}\left(\beta \hat{\gamma}^{2}\right)+T_{2^{l}}(\beta \hat{\gamma}) \\
& =T_{2^{l}}\left(\beta\left(\hat{\gamma}+\hat{\gamma}^{2}+\hat{\gamma}^{2^{l-1}}\right)\right) \\
& =L_{\hat{\gamma}+\hat{\gamma}^{2}+\hat{\gamma}^{2^{l-1}}(\beta),}
\end{aligned}
$$

which implies $\phi_{3}=L_{\hat{\gamma}+\hat{\gamma}^{2}+\hat{\gamma}^{l-1}}$.

For a given $\gamma \in \mathbb{F}_{2^{n}}$ the following proposition gives the conditions to have $W_{3}(\gamma)=$ $\mathbb{F}_{2^{l}}$, or $W_{3}(\gamma)=W_{0}$.

Proposition 59. Let $\gamma \in \mathbb{F}_{2^{n}}$. Then

(i) If $\hat{\gamma}=0$, then $W_{3}(\gamma)=\mathbb{F}_{2^{l}}$.

(ii) If $\hat{\gamma}=1$, then either $W_{3}(\gamma)=\mathbb{F}_{2^{l}}$, or $W_{3}(\gamma)=W_{0}$.

Proof. Suppose that $\gamma \in \mathbb{F}_{2^{n}}$.

(i) Let $\gamma$ be such that $\hat{\gamma}=0$. So $T_{2^{l}}(\hat{\gamma})=T_{2^{l}}(0)=0$, and Proposition 58(i) implies that $\phi_{3}=L_{0}$. Then $W_{3}(\gamma)=\operatorname{Ker}\left(\phi_{3}\right)=\operatorname{Ker}\left(L_{0}\right)=\mathbb{F}_{2^{l}}$.

(ii) Assume that $\hat{\gamma}=1$. Then if $T_{2^{l}}(\hat{\gamma})=0$, by Proposition $58(i)$ we have

$$
\phi_{3}=L_{\hat{\gamma}^{2}+\hat{\gamma}^{l-1}}=L_{1+1}=L_{0}
$$

Therefore $W_{3}(\gamma)=\mathbb{F}_{2^{l}}$. If not, then $T_{2^{l}}(\hat{\gamma})=1$, and Proposition 58(ii) implies that $\phi_{3}=L_{\hat{\gamma}+\hat{\gamma}^{2}+\hat{\gamma}^{2-1}}=L_{1}$. Thus $W_{3}(\gamma)=\operatorname{Ker}\left(\phi_{3}\right)=W_{0}$. 
In the following proposition we give the necessary and sufficient conditions for $W_{4}(\gamma)=\mathbb{F}_{2^{l}}$

Proposition 60. Let $\gamma \in \mathbb{F}_{2^{n}}$. Then $W_{4}(\gamma)=\mathbb{F}_{2^{l}}$ if and only if $\hat{\gamma}=0$, and

$$
C_{2}\left(\hat{\gamma}^{2^{l-1}}\right)+C_{3}(\hat{\gamma})=0
$$

where $C_{2}$ and $C_{3}$ are defined previously in Equation (6.2).

Proof. Let $n=4 l$, where $l=2 l^{\prime}+1$. Then by Equation (6.8) we have

$$
T_{4}(\beta+\gamma)=T_{4}(\beta)+T_{4}(\gamma)+\phi_{4}(\beta)
$$

where

$$
\phi_{4}(\beta)=\sum_{k=0}^{l^{\prime}} \sum_{i=1}^{l} \beta^{\left(1+2^{k}\right)_{i}} \delta_{1+2^{k}} 2^{i-1},
$$

and the coefficients $\delta_{1+2^{k}}$ are given as,

$$
\delta_{1+2^{k}}= \begin{cases}\hat{\gamma} \hat{\gamma}^{2^{l-1}}+\hat{\gamma}\left(\sum_{i=1}^{l-2} \sum_{j=i+1}^{l-1} \hat{\gamma}^{2^{i}} \hat{\gamma}^{2^{j}}+\sum_{i=1}^{l-1} C_{2}\left(\hat{\gamma}^{2^{i}}\right)\right) & \\ \quad+\hat{\gamma}^{2^{l-1}}\left(\sum_{i=1}^{l-2} \hat{\gamma}^{2^{i}}\right)+C_{2}\left(\hat{\gamma}^{2^{l-1}}\right)+C_{3}(\hat{\gamma}) & \text { if } k=0, \\ \hat{\gamma}+\hat{\gamma} \hat{\gamma}^{2} & \text { if } k=1, \\ \hat{\gamma} \hat{\gamma}^{2^{k}} & \text { if } k>1 .\end{cases}
$$

Suppose that $\gamma \in \mathbb{F}_{2^{n}}$ is such that $\hat{\gamma}=0$, and $C_{2}\left(\hat{\gamma}^{2-1}\right)+C_{3}(\hat{\gamma})=0$. Therefore $\delta_{1+2^{k}}=0$, for all $k$. So $\phi_{4}(\beta)=0$, for all $\beta \in \mathbb{F}_{2^{l}}$, which means

$$
W_{4}(\gamma)=\operatorname{Ker}\left(\phi_{4}\right)=\mathbb{F}_{2^{l}}
$$

Now let $W_{4}(\gamma)=\mathbb{F}_{2^{l}}$. We want to show $\hat{\gamma}=0$, and $C_{2}\left(\hat{\gamma}^{2^{l-1}}\right)+C_{3}(\hat{\gamma})=0$. Since 
$W_{4}(\gamma)=\mathbb{F}_{2^{l}}$, so $T_{4}(\beta+\gamma)=T_{4}(\beta)+T_{4}(\gamma)$, or

$$
\phi_{4}(\beta)=\sum_{k=0}^{l^{\prime}} \sum_{i=1}^{l} \beta^{\left(1+2^{k}\right)_{i}} \delta_{1+2^{k}} 2^{i-1}=0
$$

for all $\beta \in \mathbb{F}_{2^{l}}$, where $\left(1+2^{k}\right)_{i}$ refers to the $i^{\text {th }}$ element of the cyclotomic coset defined by $\left[1+2^{k}\right]$, for $0 \leq k \leq l^{\prime}$. Since the above double sum is zero, different terms are canceling pairwise. Let us take two terms of $\phi_{4}(\beta)$, like

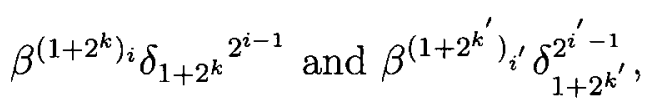

where $i \neq i^{\prime}$, or $k \neq k^{\prime}$. In these two terms the exponents of $\beta$ are from the two cyclotomic cosets $\left[1+2^{k}\right]$ and $\left[1+2^{k^{\prime}}\right]$. If $k=k^{\prime}$ and $i \neq i^{\prime}$, then the exponent of $\beta$ are two elements of the same cyclotomic coset. Since the elements of a cyclotomic coset are distinct, $\left(1+2^{k}\right)_{i} \neq\left(1+2^{k}\right)_{i^{\prime}}$ and therefore

$$
\beta^{\left(1+2^{k}\right)_{i}} \neq \beta^{\left(1+2^{k}\right)_{i}{ }^{\prime}}
$$

This implies that

$$
\beta^{\left(1+2^{k}\right)_{i}} \delta_{1+2^{k}}^{2^{i-1}}=\beta^{\left(1+2^{k}\right)_{i^{\prime}} \delta_{1+2^{k}} 2^{i^{\prime}-1}}
$$

if and only if $\delta_{1+2^{k}} 2^{i-1}=\delta_{1+2^{k}} 2^{i^{\prime}-1}$, for different $i$ and $i^{\prime}$. Thus $\delta_{1+2^{k}}=0$, for all $k$. Then from the definition of $\delta_{1+2^{k}}$, since $\delta_{1+2^{k}}=\hat{\gamma} \hat{\gamma}^{2^{k}}=0$, for $k>1$, we have $\hat{\gamma}=0$. Also for $k=0$ from $\delta_{1+2^{k}}=0$ we have $C_{2}\left(\hat{\gamma}^{2-1}\right)+C_{3}(\hat{\gamma})=0$. Similar argument apply to the other cases of $i$ and $k$.

If $l$ is even, then the proof follows the same lines as that of the proof of the odd case for $l$.

Since we do not have $\phi_{4}$ in terms of a linear functional, it is not easy to find the 
conditions for $W_{4}(\gamma)=W_{0}$. In the following propositions the necessary and sufficient conditions for different possible cases between $W_{2}$ and $W_{3}$ are given.

Proposition 61. Let $\gamma \in \mathbb{F}_{2^{n}}$, then $W_{2}(\gamma)=W_{3}(\gamma)=\mathbb{F}_{2^{l}}$ if and only if $\hat{\gamma}=0$.

Proof. Let $\gamma \in \mathbb{F}_{2^{n}}$ be such that $\hat{\gamma}=0$. Then by Propositions 56, $W_{2}(\gamma)=\mathbb{F}_{2^{l}}$. Since $T_{2^{l}}(\hat{\gamma})=T_{2^{l}}(0)=0$, then Proposition 58(i) gives $\phi_{3}=L_{0}$. Therefore $W_{3}(\gamma)=$ $\operatorname{Ker}\left(\phi_{3}\right)=\operatorname{Ker}\left(L_{0}\right)=\mathbb{F}_{2^{l}}$.

Next let $W_{2}(\gamma)=W_{3}(\gamma)=\mathbb{F}_{2^{l}}$. From $W_{2}(\gamma)=\mathbb{F}_{2^{l}}$ and Propositions 56 we have $\hat{\gamma}=0$. Moreover, if $W_{3}(\gamma)=\operatorname{Ker}\left(\phi_{3}\right)$ and $W_{3}(\gamma)=\mathbb{F}_{2^{l}}=\operatorname{Ker}\left(L_{0}\right)$, then we have $\phi_{3}=L_{0}$. Therefore Proposition 58(i) and (ii) imply $\hat{\gamma}^{2}+\hat{\gamma}^{2^{l-1}}=\hat{\gamma}+\hat{\gamma}^{2}+\hat{\gamma}^{2^{l-1}}$, which means $\hat{\gamma}=0$.

Proposition 62. If $\gamma \in \mathbb{F}_{2^{n}}$, then $W_{2}(\gamma)=W_{0}$ and $W_{3}(\gamma)=\mathbb{F}_{2^{l}}$ if and only if $\hat{\gamma}=1$ and $T_{2^{l}}(\hat{\gamma})=0$.

Proof. Let $\gamma \in \mathbb{F}_{2^{n}}$ such that $\hat{\gamma}=1$ and $T_{2^{l}}(\hat{\gamma})=0$. Since $\hat{\gamma}=1$ then by Proposition 57, $W_{2}(\gamma)=W_{0}$. Since $T_{2^{l}}(\hat{\gamma})=0$ then Proposition $58(i)$ gives

$$
\phi_{3}=L_{\hat{\gamma}^{2}+\hat{\gamma}^{l-1}}=L_{1+1}=L_{0}
$$

Therefore $\operatorname{Ker}\left(\phi_{3}\right)=\operatorname{Ker}\left(L_{0}\right)=\mathbb{F}_{2^{l}}$, which means $W_{3}(\gamma)=\mathbb{F}_{2^{l}}$.

Next, let $W_{2}(\gamma)=W_{0}$, and $W_{3}(\gamma)=\mathbb{F}_{2^{l}}$. Then by Proposition $57, \hat{\gamma}=1$. We prove that $T_{2^{l}}(\hat{\gamma})=0$. If not, then $T_{2^{l}}(\hat{\gamma})=1$, and Proposition 58(ii) gives,

$$
\phi_{3}=L_{\hat{\gamma}+\hat{\gamma}^{2}+\hat{\gamma}^{2-1}}=L_{1+1+1}=L_{1}
$$

Therefore $W_{3}=\operatorname{Ker}\left(\phi_{3}\right)=\operatorname{Ker}\left(L_{1}\right)=W_{0}$, or $W_{3}(\gamma)=W_{0}$, which is a contradiction. Thus $T_{2^{l}}(\hat{\gamma})=0$. 
Proposition 63. Let $\gamma \in \mathbb{F}_{2^{n}}$. Then $W_{2}(\gamma)=W_{0}$ and $W_{3}(\gamma)=W_{0}$ if and only if $\hat{\gamma}=1$ and $T_{2^{l}}(\hat{\gamma})=1$

Proof. Let $\gamma \in \mathbb{F}_{2^{n}}$ such that $\hat{\gamma}=1$ and $T_{2^{l}}(\hat{\gamma})=1$. Then $W_{2}(\gamma)=W_{0}$, and Proposition 58(ii) implies $W_{3}(\gamma)=\operatorname{Ker}\left(\phi_{3}\right)=\operatorname{Ker}\left(L_{1}\right)=W_{0}$.

Next, let $W_{2}(\gamma)=W_{3}(\gamma)=W_{0}$. Since $W_{2}(\gamma)=W_{0}$, we have $\hat{\gamma}=1$. We prove $T_{2^{l}}(\hat{\gamma})=1$. If not, then by Proposition 58(i), $W_{3}(\gamma)=\operatorname{Ker}\left(\phi_{3}\right)=\operatorname{Ker}\left(L_{0}\right)=\mathbb{F}_{2^{l}}$, which is a contradiction. Therefore $T_{2^{l}}(\hat{\gamma})=1$.

Proposition 64. Let $\gamma \in \mathbb{F}_{2^{n}}$ be such that $\hat{\gamma} \neq 1$. Then $W_{2}(\gamma) \neq W_{0}$ and $W_{3}(\gamma)=W_{0}$ if and only if either

(i) $T_{2^{l}}(\hat{\gamma})=0$ and $\hat{\gamma}^{4}=\hat{\gamma}+1$, or

(ii) $T_{2^{l}}(\hat{\gamma})=1$ and $\hat{\gamma}^{4}=\hat{\gamma}^{2}+\hat{\gamma}+1$.

Proof. Let $\hat{\gamma} \neq 1$. First we suppose that $W_{2}(\gamma) \neq W_{0}$ and $W_{3}(\gamma)=W_{0}$. There are two cases for $T_{2^{l}}(\hat{\gamma})$.

(i) If $T_{2^{l}}(\hat{\gamma})=0$, then by Proposition $58(i)$, we have $\phi_{3}=L_{\hat{\gamma}^{2}+\hat{\gamma}^{l-1}}$. Thus,

$$
\operatorname{Ker}\left(L_{1}\right)=W_{0}=W_{3}(\gamma)=\operatorname{Ker}\left(\phi_{3}\right)=\operatorname{Ker}\left(L_{\hat{\gamma}^{2}+\hat{\gamma}^{2-1}}\right)
$$

This implies $\hat{\gamma}^{2}+\hat{\gamma}^{2-1}=1$. So $\hat{\gamma}^{4}+\hat{\gamma}=1$, or $\hat{\gamma}^{4}=\hat{\gamma}+1$.

(ii) If $T_{2^{l}}(\hat{\gamma})=1$, then Proposition $58(i i)$ implies $\phi_{3}=L_{\hat{\gamma}+\hat{\gamma}^{2}+\hat{\gamma}^{l-1}}$. So

$$
\operatorname{Ker}\left(L_{1}\right)=W_{0}=W_{3}(\gamma)=\operatorname{Ker}\left(\phi_{3}\right)=\operatorname{Ker}\left(L_{\hat{\gamma}+\hat{\gamma}^{2}+\hat{\gamma}^{l-1}}\right) .
$$

Thus $\hat{\gamma}+\hat{\gamma}^{2}+\hat{\gamma}^{2^{l-1}}=1$, or $\hat{\gamma}^{4}+\hat{\gamma}^{2}+\hat{\gamma}=1$.

To prove the other way, let $\gamma \in \mathbb{F}_{2^{n}}$ be such that $\hat{\gamma} \neq 1$.

(i) If $T_{2^{l}}(\hat{\gamma})=0$ and $\hat{\gamma}^{4}=\hat{\gamma}+1$, we prove that $W_{2}(\gamma) \neq W_{0}$, and $W_{3}(\gamma)=W_{0}$. 
Since $\hat{\gamma}^{4}=\hat{\gamma}+1$, then $\hat{\gamma}^{4}+\hat{\gamma}=1$, or $\left(\hat{\gamma}^{4}+\hat{\gamma}\right)^{2^{l-1}}=1$. Thus $\hat{\gamma}^{2}+\hat{\gamma}^{2^{l-1}}=1$. Since $T_{2}(\hat{\gamma})=0$, so by Proposition $58(i)$ we have $\phi_{3}=L_{\hat{\gamma}^{2}+\hat{\gamma}^{l-1}}=L_{1}$. Then

$$
W_{3}(\gamma)=\operatorname{Ker}\left(\phi_{3}\right)=W_{0}
$$

Clearly $\hat{\gamma} \neq 0$, since $\hat{\gamma}=0$ contradicts $\hat{\gamma}^{4}=\hat{\gamma}+1$. Then $\hat{\gamma} \neq 0$ implies $W_{2}(\gamma) \neq \mathbb{F}_{2^{l}}$. Since $\hat{\gamma} \neq 1$, therefore $W_{2}(\gamma) \neq W_{0}$.

(ii) Let $T_{2^{l}}(\hat{\gamma})=1$ and $\hat{\gamma}^{4}=\hat{\gamma}^{2}+\hat{\gamma}+1$. We show that $W_{2}(\gamma) \neq W_{0}$, and $W_{3}(\gamma)=W_{0}$. From $\hat{\gamma}^{4}=\hat{\gamma}^{2}+\hat{\gamma}+1$ we have $\left(\hat{\gamma}^{4}+\hat{\gamma}^{2}+\hat{\gamma}\right)^{2^{l-1}}=1$, or $\hat{\gamma}+\hat{\gamma}^{2}+\hat{\gamma}^{2-1}=1$. Since $T_{2}(\hat{\gamma})=1$, then from Proposition 58(ii) we have $\phi_{3}=L_{\hat{\gamma}+\hat{\gamma}^{2}+\hat{\gamma}^{2-1}}=L_{1}$. Therefore $W_{3}(\gamma)=W_{0}$. Since $\hat{\gamma} \neq 0$, then $W_{2}(\gamma) \neq \mathbb{F}_{2^{l}}$. Also $\hat{\gamma} \neq 1$ concludes $W_{2}(\gamma) \neq W_{0}$.

Proposition 65. Let $\gamma \in \mathbb{F}_{2^{n}}$ be such that $\hat{\gamma} \neq 0,1$. Then $W_{2}(\gamma)$ and $W_{3}(\gamma)$ are hyperplanes of $\mathbb{F}_{2^{l}}$ which are different than $W_{0}$, and $W_{2}(\gamma) \cap W_{3}(\gamma) \subseteq W_{0}$ if and only if either

(i) $T_{2^{l}}(\hat{\gamma})=0$ and $\hat{\gamma}^{4}=\hat{\gamma}^{2}+\hat{\gamma}+1$, or

(ii) $T_{2^{l}}(\hat{\gamma})=1$ and $\hat{\gamma}^{4}=\hat{\gamma}+1$.

Proof. Let $\gamma \in \mathbb{F}_{2^{n}}$ and $\gamma \neq 1$.

(i) Assume $T_{2^{l}}(\hat{\gamma})=0$, and $\hat{\gamma}^{4}=\hat{\gamma}^{2}+\hat{\gamma}+1$. This condition implies that $\hat{\gamma} \neq 0$, or $W_{2}(\gamma) \neq \mathbb{F}_{2^{l}}$. Also $\hat{\gamma} \neq 1$ gives $W_{2} \neq W_{0}$. From $\hat{\gamma}^{4}=\hat{\gamma}^{2}+\hat{\gamma}+1$ we have $\hat{\gamma}^{4}+\hat{\gamma}^{2}+\hat{\gamma}^{2^{l-1}}=1$, or $\hat{\gamma}^{2^{l-1}}+\hat{\gamma}^{2}+\hat{\gamma}=1$. Since $T_{2}(\hat{\gamma})=0$, then Proposition 58(i) gives $\phi_{3}=L_{\hat{\gamma}^{2}+\hat{\gamma}^{2-1}}$. Therefore

$$
\phi_{2}+\phi_{3}=L_{\hat{\gamma}}+L_{\hat{\gamma}^{2}+\hat{\gamma}^{l-1}}=L_{\hat{\gamma}+\hat{\gamma}^{2}+\hat{\gamma}^{l-1}}=L_{1}
$$


and

$$
W_{2}(\gamma) \cap W_{3}(\gamma)=\operatorname{Ker}\left(\phi_{2}\right) \cap \operatorname{Ker}\left(\phi_{3}\right) \subseteq \operatorname{Ker}\left(L_{1}\right)=W_{0}
$$

So $W_{2}(\gamma) \cap W_{3}(\gamma) \subseteq W_{0}$. If $W_{3}(\gamma)=W_{0}$, then $\phi_{3}=L_{1}$, and by Proposition $58(i)$ we have $\phi_{3}=L_{\hat{\gamma}^{2}+\hat{\gamma}^{2-1}}$. Therefore $\hat{\gamma}^{2}+\hat{\gamma}^{2-1}=1$, or $\hat{\gamma}^{4}+\hat{\gamma}=1$. Then $\hat{\gamma}^{4}=\hat{\gamma}^{2}+\hat{\gamma}+1$ means $\hat{\gamma}=0$, which is a contradiction. So $W_{3}(\gamma) \neq W_{0}$. If $W_{3}(\gamma)=\mathbb{F}_{2^{l}}$, then $\phi_{3}=L_{0}$. Since $T_{2^{l}}(\hat{\gamma})=0$, so $\phi_{3}=L_{\hat{\gamma}^{2}+\hat{\gamma}^{2 l-1}}$. Therefore $\hat{\gamma}^{2}+\hat{\gamma}^{2^{l-1}}=0$, or $\hat{\gamma}^{4}+\hat{\gamma}=0$. Then $\hat{\gamma}^{4}=\hat{\gamma}^{2}+\hat{\gamma}+1$ implies $\hat{\gamma}=1$, which is a contradiction. So $W_{3}(\gamma) \neq \mathbb{F}_{2^{l}}$.

(ii) Let $T_{2^{l}}(\hat{\gamma})=1$ and $\hat{\gamma}^{4}=\hat{\gamma}+1$. Then $\left(\hat{\gamma}^{4}+\hat{\gamma}\right)^{2^{l-1}}=1$, or $\hat{\gamma}^{2}+\hat{\gamma}^{2^{l-1}}=1$. Since $T_{2^{l}}(\hat{\gamma})=1$, Proposition $58(i)$ implies $\phi_{3}=L_{\hat{\gamma}+\hat{\gamma}^{2}+\hat{\gamma}^{l-1}}$. Then,

$$
\phi_{2}+\phi_{3}=L_{\hat{\gamma}}+L_{\hat{\gamma}+\hat{\gamma}^{2}+\hat{\gamma}^{2 l-1}}=L_{\hat{\gamma}^{2}+\hat{\gamma}^{2 l-1}}=L_{1}
$$

Moreover,

$$
W_{2}(\gamma) \cap W_{3}(\gamma)=\operatorname{Ker}\left(\phi_{2}\right) \cap \operatorname{Ker}\left(\phi_{3}\right) \subseteq \operatorname{Ker}\left(L_{1}\right)=W_{0}
$$

or $W_{2}(\gamma) \cap W_{3}(\gamma) \subseteq W_{0}$. From the condition $\hat{\gamma}^{4}=\hat{\gamma}+1$ we have $\hat{\gamma} \neq 0$, or $W_{2}(\gamma) \neq \mathbb{F}_{2^{l}}$. Also $W_{2} \neq W_{0}$, since $\hat{\gamma} \neq 1$. Using Proposition $58(i)$, the assumption $T_{2^{l}}(\hat{\gamma})=1$ gives

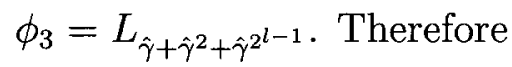

$$
W_{3}(\gamma)=\operatorname{Ker}\left(\phi_{3}\right)=\operatorname{Ker}\left(L_{\hat{\gamma}+\hat{\gamma}^{2}+\hat{\gamma}^{2 l-1}}\right)
$$

If $W_{3}(\gamma)=\mathbb{F}_{2^{l}}=\operatorname{Ker}\left(L_{0}\right)$ then $\hat{\gamma}+\hat{\gamma}^{2}+\hat{\gamma}^{2^{l-1}}=0$, and $\left(\hat{\gamma}+\hat{\gamma}^{2}+\hat{\gamma}^{2^{l-1}}\right)^{2}=0$, or $\hat{\gamma}^{4}+\hat{\gamma}^{2}+\hat{\gamma}=0$. This equation and the condition $\hat{\gamma}^{4}=\hat{\gamma}+1$ imply $\hat{\gamma}=1$, which is a contradiction. So $W_{3}(\gamma) \neq \mathbb{F}_{2^{l}}$. If $W_{3}(\gamma)=W_{0}=\operatorname{Ker}\left(L_{1}\right)$, then $\hat{\gamma}+\hat{\gamma}^{2}+\hat{\gamma}^{2-1}=1$. Thus $\hat{\gamma}^{4}+\hat{\gamma}^{2}+\hat{\gamma}=1$, and from $\hat{\gamma}^{4}=\hat{\gamma}+1$ we have $\hat{\gamma}=0$, which is contradiction. Therefore $W_{3}(\gamma) \neq W_{0}$. 
Now let us prove the other way. Let $W_{2}(\gamma) \neq W_{0}$ and $W_{3}(\gamma) \neq W_{0}$ be such that $W_{2}(\gamma) \cap W_{3}(\gamma) \subseteq W_{0}$. We prove $(i)$ and $(i i)$. If $T_{2^{l}}(\hat{\gamma})=0$ then by Proposition 58(i) we have $\phi_{3}=L_{\hat{\gamma}^{2}+\hat{\gamma}^{2-1}}$. Therefore

$$
W_{3}(\gamma)=\operatorname{Ker}\left(\phi_{3}\right)=\operatorname{Ker}\left(L_{\hat{\gamma}^{2}+\hat{\gamma}^{l-1}}\right)
$$

and also $W_{2}(\gamma)=\operatorname{Ker}\left(L_{\hat{\gamma}}\right)$. For any $\beta \in W_{0}=\operatorname{Ker}\left(L_{1}\right)$ either

$$
\beta \in W_{2}(\gamma) \cap W_{3}(\gamma)=\operatorname{Ker}\left(L_{\hat{\gamma}}\right) \cap \operatorname{Ker}\left(L_{\hat{\gamma}^{2}+\hat{\gamma}^{2-1}}\right),
$$

or $\beta \notin W_{2}(\gamma) \cap W_{3}(\gamma)$. If $\beta \in \operatorname{Ker}\left(L_{\hat{\gamma}}\right) \cap \operatorname{Ker}\left(L_{\hat{\gamma}^{2}+\hat{\gamma}^{2-1}}\right)$, then

$$
T_{2^{l}}(\beta \hat{\gamma})+T_{2^{l}}\left(\beta\left(\hat{\gamma}^{2}+\hat{\gamma}^{2^{l-1}}\right)\right)=0, \text { or } T_{2^{l}}\left(\beta\left(\hat{\gamma}+\hat{\gamma}^{2}+\hat{\gamma}^{2^{l-1}}\right)\right)=0
$$

Thus $\beta \in \operatorname{Ker}\left(L_{\hat{\gamma}+\hat{\gamma}^{2}+\hat{\gamma}^{l-1}}\right)$, which means $\operatorname{Ker}\left(L_{1}\right) \subseteq \operatorname{Ker}\left(L_{\hat{\gamma}+\hat{\gamma}^{2}+\hat{\gamma}^{l-1}}\right)$.

If $\hat{\gamma}+\hat{\gamma}^{2}+\hat{\gamma}^{2^{l-1}}=0$, then $\hat{\gamma}=\hat{\gamma}^{2}+\hat{\gamma}^{2^{l-1}}$, and so $\operatorname{Ker}\left(L_{\hat{\gamma}}\right)=\operatorname{Ker}\left(L_{\hat{\gamma}^{2}+\hat{\gamma}^{l-1}}\right)$. Then

$$
\operatorname{Ker}\left(L_{\hat{\gamma}}\right)=\operatorname{Ker}\left(L_{\hat{\gamma}}\right) \cap \operatorname{Ker}\left(L_{\hat{\gamma}^{2}+\hat{\gamma}^{2-1}}\right)=W_{2}(\gamma) \cap W_{3}(\gamma) \subseteq W_{0}=\operatorname{Ker}\left(L_{1}\right)
$$

So $\operatorname{Ker}\left(L_{\hat{\gamma}}\right) \subseteq \operatorname{Ker}\left(L_{1}\right)$, and therefore $\hat{\gamma}=1$, which is a contradiction. This gives $\hat{\gamma}+\hat{\gamma}^{2}+\hat{\gamma}^{2^{l-1}}=1$, or $\hat{\gamma}^{4}+\hat{\gamma}^{2}+\hat{\gamma}=1$. In a similar way we can show that if $\beta \notin W_{2}(\gamma) \cap W_{3}(\gamma)$, then $\hat{\gamma}^{4}=\hat{\gamma}^{2}+\hat{\gamma}+1$. This proves $(i)$.

Now let $T_{2^{l}}(\hat{\gamma})=1$. Clearly, $W_{2}(\gamma)=\operatorname{Ker}\left(L_{\hat{\gamma}}\right)$. By Proposition 58(ii) we have

$$
W_{3}(\gamma)=\operatorname{Ker}\left(\phi_{3}\right)=\operatorname{Ker}\left(L_{\hat{\gamma}+\hat{\gamma}^{2}+\hat{\gamma}^{l-1}}\right)
$$

Again, for any $\beta \in W_{0}$ we have $\beta \in W_{2}(\gamma) \cap W_{3}(\gamma)$, or $\beta \notin W_{2}(\gamma) \cap W_{3}(\gamma)$. If 
$\beta \in W_{2}(\gamma) \cap W_{3}(\gamma)$, then $\beta \in \operatorname{Ker}\left(L_{\hat{\gamma}}\right) \cap \operatorname{Ker}\left(L_{\hat{\gamma}+\hat{\gamma}^{2}+\hat{\gamma}^{l-1}}\right)$. Thus,

$$
T_{2^{l}}(\beta \hat{\gamma})+T_{2^{l}}\left(\beta\left(\hat{\gamma}+\hat{\gamma}^{2}+\hat{\gamma}^{2^{l-1}}\right)\right)=T_{2^{l}}\left(\beta\left(\hat{\gamma}+\hat{\gamma}+\hat{\gamma}^{2}+\hat{\gamma}^{2^{l-1}}\right)\right)=0
$$

Then $T_{2^{l}}\left(\beta\left(\hat{\gamma}^{2}+\hat{\gamma}^{l-1}\right)\right)=0$, or $\beta \in \operatorname{Ker}\left(L_{\hat{\gamma}^{2}+\hat{\gamma}^{2-1}}\right)$. This means

$$
W_{0}=\operatorname{Ker}\left(L_{1}\right) \subseteq \operatorname{Ker}\left(L_{\hat{\gamma}^{2}+\hat{\gamma}^{l-1}}\right)
$$

If we can show that $\hat{\gamma}^{2}+\hat{\gamma}^{l-1} \neq 0$, then from $\operatorname{Ker}\left(L_{1}\right) \subseteq \operatorname{Ker}\left(L_{\hat{\gamma}^{2}+\hat{\gamma}^{2-1}}\right)$ we have $\hat{\gamma}^{2}+\hat{\gamma}^{2^{l-1}}=1$, or $\hat{\gamma}^{4}=\hat{\gamma}+1$.

To prove $\hat{\gamma}^{2}+\hat{\gamma}^{2^{l-1}} \neq 0$ we use a contradiction. If $\hat{\gamma}^{2}+\hat{\gamma}^{2^{l-1}}=0$, then $\hat{\gamma}^{4}+\hat{\gamma}=0$, and $L_{\hat{\gamma}^{4}+\hat{\gamma}}=L_{0}$. Therefore $\operatorname{Ker}\left(L_{\hat{\gamma}^{4}+\hat{\gamma}}\right)=\operatorname{Ker}\left(L_{0}\right)=\mathbb{F}_{2^{t}}$. Moreover,

$$
W_{2}(\gamma)=\operatorname{Ker}\left(L_{\hat{\gamma}}\right)=\operatorname{Ker}\left(L_{\hat{\gamma}}\right) \cap \mathbb{F}_{2^{l}}=\operatorname{Ker}\left(L_{\hat{\gamma}}\right) \cap \operatorname{Ker}\left(L_{0}\right)
$$

Then from $W_{2}(\gamma)=\operatorname{Ker}\left(L_{\hat{\gamma}}\right)$ and $\operatorname{Ker}\left(L_{\hat{\gamma}^{4}+\hat{\gamma}}\right)=\operatorname{Ker}\left(L_{0}\right)$ we have

$$
\operatorname{Ker}\left(L_{\hat{\gamma}}\right)=\operatorname{Ker}\left(L_{\hat{\gamma}}\right) \cap \operatorname{Ker}\left(L_{\hat{\gamma}^{4}+\hat{\gamma}}\right)=W_{2}(\gamma) \cap W_{3}(\gamma) \subseteq W_{0}=\operatorname{Ker}\left(L_{1}\right)
$$

or $\operatorname{Ker}\left(L_{\hat{\gamma}}\right) \subseteq W_{0}=\operatorname{Ker}\left(L_{1}\right)$, which implies $\hat{\gamma}=1$. Thus $W_{2}(\gamma)=W_{0}$, while we have $W_{2}(\gamma) \neq W_{0}$. This contradiction implies that $\hat{\gamma}^{2}+\hat{\gamma}^{2^{l-1}} \neq 0$, and therefore $\hat{\gamma}^{4}=\hat{\gamma}+1$.

Next let $\beta \in W_{0}=\operatorname{Ker}\left(L_{1}\right)$ be such that $\beta \notin W_{2}(\gamma) \cap W_{3}(\gamma)$. Then we show that $\hat{\gamma}^{4}=\hat{\gamma}+1$. Since $\beta \notin W_{2}(\gamma) \cap W_{3}(\gamma)$, we have

$$
T_{2^{l}}(\beta \hat{\gamma})+T_{2^{l}}\left(\beta\left(\hat{\gamma}+\hat{\gamma}^{2}+\hat{\gamma}^{l^{l-1}}\right)\right)=1+1=0 .
$$


Thus $T_{2^{l}}\left(\beta\left(\hat{\gamma}^{2}+\hat{\gamma}^{2^{l-1}}\right)\right)=0$, or $\beta \in \operatorname{Ker}\left(L_{\hat{\gamma}^{2}+\hat{\gamma}^{l-1}}\right)$.

The rest of the proof is the same as the case $\beta \in W_{2}(\gamma) \cap W_{3}(\gamma)$.

Let $\gamma \in \mathbb{F}_{2^{n}}$ be given. Then $\gamma+\mathbb{F}_{2^{l}}$ is a coset of $\mathbb{F}_{2^{n}}$, and $\gamma+\beta$ represents an element of this coset, where $\beta \in \mathbb{F}_{2^{l}}$. For any $\beta \in \mathbb{F}_{2^{l}}$, either $\beta \in W_{i}(\gamma)$, or $\beta \notin W_{i}(\gamma)$, where $i=2,3,4$. In each of these situations the following proposition gives the traces of $\gamma+\beta$.

Proposition 66. Let $\gamma \in \mathbb{F}_{2^{n}}$, and $\beta \in \mathbb{F}_{2^{l}}$,

$$
T_{2}(\gamma+\beta)= \begin{cases}T_{2}(\gamma) & \text { if } \beta \in W_{2}(\gamma) \\ T_{2}(\gamma)+1 & \text { if } \beta \notin W_{2}(\gamma)\end{cases}
$$

(ii)

$$
T_{3}(\gamma+\beta)= \begin{cases}T_{3}(\gamma) & \text { if } \beta \in W_{3}(\gamma) \\ T_{3}(\gamma)+1 & \text { if } \beta \notin W_{3}(\gamma)\end{cases}
$$

(iii)

$$
T_{4}(\gamma+\beta)= \begin{cases}T_{4}(\gamma)+T_{2^{l}}(\beta) & \text { if } \beta \in W_{4}(\gamma), \\ T_{4}(\gamma)+T_{2^{l}}(\beta)+1 & \text { if } \beta \notin W_{4}(\gamma) .\end{cases}
$$

Proof. Let $\gamma \in \mathbb{F}_{2^{n}}$, and $\beta \in \mathbb{F}_{2^{l}}$. Therefore by Proposition $54, T_{i}(\beta)=0$, where $i=1,2,3$, and $T_{4}(\beta)=T_{2^{l}}(\beta)$. Thus,

(i) By Equation (6.3) for $\beta \in \mathbb{F}_{2^{l}}$ we have

$$
T_{2}(\beta+\gamma)=T_{2}(\beta)+T_{2}(\gamma)+T_{2^{l}}(\beta \hat{\gamma})
$$

If $\beta \in W_{2}(\gamma)$, then

$$
T_{2}(\gamma+\beta)=T_{2}(\gamma)+T_{2}(\beta)=T_{2}(\gamma)
$$

So $T_{2^{l}}(\beta \hat{\gamma})=\phi_{2}(\beta)=0$. If $\beta \notin W_{2}(\gamma)$, then $\phi_{2}(\beta)=T_{2^{l}}(\beta \hat{\gamma})=1$, and by Equa- 
tion (6.3),

$$
T_{2}(\gamma+\beta)=T_{2}(\gamma)+T_{2^{l}}(\beta \hat{\gamma})=T_{2}(\gamma)+1
$$

(ii) For $\beta \in \mathbb{F}_{2^{l}}$ Equation (6.4) implies that

$$
T_{3}(\beta+\gamma)=T_{3}(\beta)+T_{3}(\gamma)+T_{2^{l}}\left(\beta^{2} \hat{\gamma}+\beta \hat{\gamma}^{2}+\beta \hat{\gamma} T_{2^{l}}(\hat{\gamma})\right)
$$

Let $\beta \in W_{3}(\gamma)$, then

$$
T_{3}(\gamma+\beta)=T_{3}(\gamma)+T_{3}(\beta)=T_{3}(\gamma)
$$

Therefore $T_{2^{l}}\left(\beta^{2} \hat{\gamma}+\beta \hat{\gamma}^{2}+\beta \hat{\gamma} T_{2^{l}}(\hat{\gamma})\right)=\phi_{3}(\beta)=0$. If $\beta \notin W_{3}(\gamma)$, then $\phi_{3}(\beta)=1$, and

$$
T_{3}(\beta+\gamma)=T_{3}(\beta)+T_{3}(\gamma)+\phi_{3}(\beta)=T_{3}(\gamma)+1
$$

(iii) We have $n=4 l$, and let $l$ be even. If $\beta \in W_{4}(\gamma)$, then by Equation (6.5) we have $\phi_{4}(\beta)=0$ and

$$
T_{4}(\gamma+\beta)=T_{4}(\gamma)+T_{4}(\beta)=T_{4}(\gamma)+T_{2^{l}}(\beta)
$$

If $\beta \notin W_{4}(\gamma)$, then $\phi_{4}(\beta)=1$, and $T_{4}(\gamma+\beta)=T_{4}(\gamma)+T_{2^{l}}(\beta)+1$.

For $\gamma \in \mathbb{F}_{2^{n}}$ we can define the coset $\gamma+\mathbb{F}_{2^{n}}$ of $\mathbb{F}_{2^{l}}$ in $\mathbb{F}_{2^{n}}$. Moreover, we prove that under different conditions on $\gamma$ we expect different situations for $W_{i}(\gamma)$, where $i=2,3,4$. Either $W_{i}(\gamma)=\mathbb{F}_{2^{l}}$ or $W_{i}(\gamma)=W_{0}$ or $W_{i}(\gamma)$ is a hyperplane of $\mathbb{F}_{2^{l}}$ such that $W_{i}(\gamma) \neq W_{0}$. For example, if $\gamma \in \mathbb{F}_{2^{n}}$ is such that $\hat{\gamma}=0$ and $C_{2}\left(\hat{\gamma}^{2^{l-1}}\right)+C_{3}(\hat{\gamma})=0$ that are given in Proposition 60, then $W_{i}(\gamma)=\mathbb{F}_{2^{l}}$, where $i=2,3$, 4. Since $T_{1}$ is linear then for any $\gamma \in \mathbb{F}_{2^{n}}$ we have $W_{1}(\gamma)=\mathbb{F}_{2^{l}}$. 


\subsection{Traces in Different Categories}

Let $\gamma \in \mathbb{F}_{2^{n}}$ be given. In this section we categorize different types of cosets $\gamma+\mathbb{F}_{2^{l}}$, where $n=4 l$. The difference between different types of cosets $\gamma+\mathbb{F}_{2^{l}}$ is given by $W_{2}(\gamma), W_{3}(\gamma)$ and $W_{4}(\gamma)$. We remind that for $\gamma \in \mathbb{F}_{2^{n}}$ either $W_{i}(\gamma)=\mathbb{F}_{2^{l}}$ or $W_{0}$ or $W_{i}(\gamma) \neq W_{0}$. In total there exist 27 different cases among $W_{2}(\gamma), W_{3}(\gamma)$ and $W_{4}(\gamma)$. Let us recall that $W_{1}(\gamma)=\mathbb{F}_{2^{l}}$, for all $\gamma \in \mathbb{F}_{2^{n}}$. Earlier we proved that some of these cases are possible, and in the previous section the conditions for $\gamma$ in those case are given. For each of the possible cases we introduce a category of cosets $\gamma+\mathbb{F}_{2^{l}}$. Then for a given coset $\gamma+\mathbb{F}_{2^{l}}$, from each of the categories, we study the traces of an element $\gamma+\beta$, where $\beta \in \mathbb{F}_{2^{l}}$.

To analyze the possible cases we divide them into three groups, based on $W_{2}(\gamma)$. Then we give the different categories of cosets.

Group One: $W_{2}(\gamma)=\mathbb{F}_{2^{l}}$.

By Proposition 56, $W_{2}(\gamma)=W_{3}(\gamma)=\mathbb{F}_{2^{l}}$ if and only if $\hat{\gamma}=0$. Also by Proposition 60 we have $W_{4}(\gamma)=\mathbb{F}_{2^{l}}$ if and only if $\hat{\gamma}=0$ and $C_{2}\left(\hat{\gamma}^{2^{l-1}}\right)+C_{3}(\hat{\gamma})=0$, such that $C_{2}$ and $C_{3}$ are defined in Section 6.1. Otherwise $W_{4}(\gamma)=W_{0}$, or $W_{4}(\gamma)$ is a hyperplane of $\mathbb{F}_{2^{l}}$, different from $W_{0}$. Therefore in this group for a given $\gamma \in \mathbb{F}_{2^{n}}$ we have the following three categories of cosets:

$$
\begin{aligned}
& \text { Category } \mathrm{A}=\left\{\gamma+\mathbb{F}_{2^{l}} \mid W_{2}=W_{3}=W_{4}=\mathbb{F}_{2^{l}}\right\}, \\
& \text { Category } \mathrm{A}_{1}=\left\{\gamma+\mathbb{F}_{2^{l}} \mid W_{2}=W_{3}=\mathbb{F}_{2^{l}}, W_{4}=W_{0}\right\}, \\
& \text { Category } \mathrm{A}_{2}=\left\{\gamma+\mathbb{F}_{2^{l}} \mid W_{2}=W_{3}=\mathbb{F}_{2^{l}}, W_{4} \neq W_{0}, \mathbb{F}_{2^{l}}\right\} .
\end{aligned}
$$

Then Table 6.1 gives all the possible categories in this group.

Group Two: $W_{2}(\gamma)=W_{0}$. 
Table 6.1: Group one: $W_{2}(\gamma)=\mathbb{F}_{2^{l}}$.

\begin{tabular}{|c|c|c|c|}
\hline$W_{4}$ & $\mathbb{F}_{2^{l}}$ & $W_{0}$ & $W_{3} \neq W_{0}, \mathbb{F}_{2^{l}}$ \\
\hline $\mathbb{F}_{2^{l}}$ & Cat A & N/A & N/A \\
\hline$W_{0}$ & Cat $\mathrm{A}_{1}$ & N/A & N/A \\
\hline$W_{4} \neq W_{0}, \mathbb{F}_{2^{l}}$ & Cat $\mathrm{A}_{2}$ & N/A & N/A \\
\hline
\end{tabular}

Since $W_{2}(\gamma)=W_{0}$, by Proposition 57 in this group $\hat{\gamma}=1$. Then by Propositions 62 and 63 we have $W_{3}(\gamma)=\mathbb{F}_{2^{l}}$, or $W_{3}(\gamma)=W_{0}$. Moreover, since $\hat{\gamma} \neq 0$, so $W_{4}(\gamma) \neq \mathbb{F}_{2^{l}}$. So for a given $\gamma \in \mathbb{F}_{2^{n}}$, in this group we have the following four categories of cosets $\gamma+\mathbb{F}_{2^{l}}$ :

$$
\begin{aligned}
& \text { Category } \mathrm{B}_{1}=\left\{\gamma+\mathbb{F}_{2^{l}} \mid W_{2}=W_{4}=W_{0}, W_{3}=\mathbb{F}_{2^{l}}\right\}, \\
& \text { Category } \mathrm{B}_{2}=\left\{\gamma+\mathbb{F}_{2^{l}} \mid W_{2}=W_{0}, W_{3}=\mathbb{F}_{2^{l}}, W_{4} \neq W_{0}, \mathbb{F}_{2^{l}}\right\} \\
& \text { Category } \mathrm{C}_{1}=\left\{\gamma+\mathbb{F}_{2^{l}} \mid W_{2}=W_{3}=W_{4}=W_{0}\right\} \\
& \text { Category } \mathrm{C}_{2}=\left\{\gamma+\mathbb{F}_{2^{l}} \mid W_{2}=W_{3}=W_{0}, W_{4} \neq W_{0}, \mathbb{F}_{2^{l}}\right\} .
\end{aligned}
$$

Different categories of the second group are given in Table 6.2.

Table 6.2: Group two: $W_{2}(\gamma)=W_{0}$.

\begin{tabular}{|c|c|c|c|}
\hline$W_{4}$ & $\mathbb{F}_{2^{l}}$ & $W_{0}$ & $W_{3} \neq W_{0}, \mathbb{F}_{2^{l}}$ \\
\hline $\mathbb{F}_{2^{l}}$ & N/A & N/A & N/A \\
\hline$W_{0}$ & Cat B $B_{1}$ & Cat $C_{1}$ & N/A \\
\hline$W_{4} \neq W_{0}, \mathbb{F}_{2^{l}}$ & Cat $B_{2}$ & Cat $C_{2}$ & N/A \\
\hline
\end{tabular}

Group Three: $W_{2}(\gamma) \neq W_{0}, \mathbb{F}_{2^{l}}$.

Since $W_{2}(\gamma)$ is a hyperplane different from $W_{0}$, we have $\hat{\gamma} \neq 0,1$ and therefore in this group $W_{4}(\gamma) \neq \mathbb{F}_{2^{l}}$. Also by Propositions 64 and 65 either $W_{3}(\gamma)=W_{0}$ or $W_{3}(\gamma) \neq W_{0}$. So in this group, for any $\gamma \in \mathbb{F}_{2^{n}}$ the coset $\gamma+\mathbb{F}_{2^{l}}$ can be in one of the 
following categories:

$$
\begin{aligned}
& \text { Category } \mathrm{D}_{1}=\left\{\gamma+\mathbb{F}_{2^{l}} \mid W_{2} \neq W_{0}, \mathbb{F}_{2^{l}}, W_{3}=W_{4}=W_{0}\right\}, \\
& \text { Category } \mathrm{D}_{2}=\left\{\gamma+\mathbb{F}_{2^{l}} \mid W_{2}, W_{4} \neq W_{0}, \mathbb{F}_{2^{l}}, W_{3}=W_{0}\right\}, \\
& \text { Category } \mathrm{E}_{1}=\left\{\gamma+\mathbb{F}_{2^{l}} \mid W_{2}, W_{3} \neq W_{0}, \mathbb{F}_{2^{l}}, W_{4}=W_{0}\right\}, \\
& \text { Category } \mathrm{E}_{2}=\left\{\gamma+\mathbb{F}_{2^{l}} \mid W_{2}, W_{3}, W_{4} \neq W_{0}, \mathbb{F}_{2^{l}}\right\} .
\end{aligned}
$$

Let the rest of possible cases for categories be Cat $\left(\mathrm{F}_{1}\right)$ and $\operatorname{Cat}\left(\mathrm{F}_{2}\right)$, which are defined as:

$$
\begin{aligned}
& \text { Category } \mathrm{F}_{1}=\left\{\gamma+\mathbb{F}_{2^{l}} \mid W_{2} \neq W_{0}, \mathbb{F}_{2^{l}}, W_{3}=\mathbb{F}_{2^{l}}, W_{4}=W_{0}\right\} \\
& \text { Category } \mathrm{F}_{2}=\left\{\gamma+\mathbb{F}_{2^{l}} \mid W_{2}, W_{4} \neq W_{0}, \mathbb{F}_{2^{l}}, W_{3}=\mathbb{F}_{2^{l}}\right\} .
\end{aligned}
$$

In Table 6.3 we have possible categories of group three.

Table 6.3: Group three: $W_{2}(\gamma) \neq W_{0}, \mathbb{F}_{2^{l}}$.

\begin{tabular}{|c|c|c|c|}
\hline$W_{4}$ & $\mathbb{F}_{2^{l}}$ & $W_{0}$ & $W_{3} \neq W_{0}, \mathbb{F}_{2^{l}}$ \\
\hline $\mathbb{F}_{2^{l}}$ & $\mathrm{~N} / \mathrm{A}$ & N/A & N/A \\
\hline$W_{0}$ & Cat $\mathrm{F}_{1}$ & Cat $\mathrm{D}_{1}$ & Cat $\mathrm{E}_{1}$ \\
\hline$W_{4} \neq W_{0}, \mathbb{F}_{2^{l}}$ & Cat $\mathrm{F}_{2}$ & Cat $\mathrm{D}_{2}$ & Cat $\mathrm{E}_{2}$ \\
\hline
\end{tabular}

For a given $\gamma \in \mathbb{F}_{2^{n}}$ the coset $\gamma+\mathbb{F}_{2^{l}}$ is in one of the above thirteen categories. In the following, for a given category we find different traces of an element $\gamma+\beta$ from the coset $\gamma+\mathbb{F}_{2^{l}}$, where $\beta \in \mathbb{F}_{2^{l}}$. In the special case if $\gamma=0$, then clearly $\hat{\gamma}=0$ and the coset is in one of the categories $A, A_{1}$ or $A_{2}$. Indeed the coset is $\gamma+\mathbb{F}_{2^{l}}=0+\mathbb{F}_{2^{l}}=\mathbb{F}_{2^{l}}$. Now we compute traces in different categories.

\section{Category A.}


For any $\beta \in \mathbb{F}_{2^{l}}$ either $\beta \in W_{0}$, or $\beta \notin W_{0}$. Since $W_{2}=W_{3}=W_{4}=\mathbb{F}_{2^{l}}$, then by Propositions 54 and 66 the traces of an element $0+\beta$ from the coset $0+\mathbb{F}_{2^{l}}$ can be given as

$$
\begin{aligned}
& T_{1}(0+\beta)=T_{1}(0)+T_{1}(\beta)=0+0=0, \\
& T_{2}(0+\beta)=T_{2}(0)+T_{2}(\beta)=0+0=0, \\
& T_{3}(0+\beta)=T_{3}(0)+T_{3}(\beta)=0+0=0, \\
& T_{4}(0+\beta)=T_{4}(0)+T_{2^{l}}(\beta)=0+T_{2^{l}}(\beta) .
\end{aligned}
$$

If $\beta \in W_{0}$, then $T_{2^{l}}(\beta)=0$ and therefore $T_{4}(0+\beta)=0$. Otherwise $T_{4}(0+\beta)=1$. So for half of the elements of the coset $0+\mathbb{F}_{2^{l}}$ the traces are $(0,0,0,0)$, and for the other

\begin{tabular}{|c|c|c|c|c|}
\hline$\sum_{\beta \in \mathbb{F}_{2^{l}}} T_{i}(0+\beta)$ & $T_{1}(0+\beta)$ & $T_{2}(0+\beta)$ & $T_{3}(0+\beta)$ & $T_{4}(0+\beta)$ \\
\hline$\beta \in W_{0}$ & 0 & 0 & 0 & 0 \\
\hline$\beta \in \mathbb{F}_{2^{l}} \backslash W_{0}$ & 0 & 0 & 0 & 1 \\
\hline
\end{tabular}
half of the elements the traces are $(0,0,0,1)$. Table 6.4 gives traces in the category A.

Table 6.4: Traces of a coset in Category A.

\section{Category $\mathbf{A}_{1}$.}

In this category $W_{2}=W_{3}=\mathbb{F}_{2^{l}}$, and $W_{4}=W_{0}$. Using Propositions 54 and 66 the traces of an element $0+\beta$ from the $\operatorname{coset} 0+\mathbb{F}_{2^{l}}$ are

$$
\begin{aligned}
& T_{1}(0+\beta)=T_{1}(0)+T_{1}(\beta)=0+0=0 \\
& T_{2}(0+\beta)=T_{2}(0)+T_{2}(\beta)=0+0=0, \\
& T_{3}(0+\beta)=T_{3}(0)+T_{3}(\beta)=0+0=0 .
\end{aligned}
$$

If $\beta \in W_{4}=W_{0}$ then $T_{2^{l}}(\beta)=0$, and $T_{4}(0+\beta)=T_{4}(0)+T_{2^{l}}(\beta)=T_{2^{l}}(\beta)=0$, 
otherwise $T_{2^{l}}(\beta)=1$ and $T_{4}(0+\beta)=1$. So for half of the elements of the coset $0+\mathbb{F}_{2^{l}}$ the traces are $(0,0,0,0)$, and for the other half of the elements the traces are $(0,0,0,1)$. We have Table 6.5 for different traces in the category $A_{1}$.

Table 6.5: Traces of a coset in Category $\mathrm{A}_{1}$.

\begin{tabular}{|c|c|c|c|c|}
\hline$\sum_{\beta \in \mathbb{F}_{2^{l}}} T_{i}(0+\beta)$ & $T_{1}(0+\beta)$ & $T_{2}(0+\beta)$ & $T_{3}(0+\beta)$ & $T_{4}(0+\beta)$ \\
\hline$\beta \in W_{0}=W_{4}$ & $\overline{0}$ & 0 & 0 & 0 \\
\hline$\beta \in \mathbb{F}_{2^{l}} \backslash W_{0}$ & 0 & 0 & 0 & 1 \\
\hline
\end{tabular}

\section{Category $\mathbf{A}_{2}$.}

We have $W_{2}=W_{3}=\mathbb{F}_{2^{l}}$, and $W_{4} \neq W_{0}$. Then by Propositions 54 and $66, T_{i}(0+\beta)=$ 0 , for $i=1,2,3$. Since $W_{4} \neq W_{0}$, we can divide $\mathbb{F}_{2^{l}}$ into four subspaces of dimension $l-2$, which are $W_{4} \cap W_{0}, W_{4} \backslash\left(W_{4} \cap W_{0}\right), W_{0} \backslash\left(W_{4} \cap W_{0}\right)$, and $\mathbb{F}_{2^{l}} \backslash\left(W_{4} \cup W_{0}\right)$. Therefore each $\beta \in \mathbb{F}_{2^{l}}$ can be in one of these four subspaces, and then we can find $T_{4}(0+\beta)$. For example, if $W_{4} \backslash\left(W_{4} \cap W_{0}\right)$, then $\beta \in W_{4}$ and $\beta \notin W_{0}$, so $T_{2^{2}}(\beta)=1$, and

$$
T_{4}(0+\beta)=T_{4}(0)+T_{4}(\beta)=1 .
$$

Similarly for the other three subspaces we can find $T_{4}(0+\beta)$. Therefore the traces of an element $0+\beta$ from the coset $0+\mathbb{F}_{2^{l}}$ in this category are given as $\left(0,0,0, T_{2^{l}}(\beta)\right)$. This means in each coset of this category, for half of the elements the traces are $(0,0,0,0)$, and the other half of the elements the traces are $(0,0,0,1)$. Table 6.6 gives the traces in the category $\mathrm{A}_{2}$.

Table 6.6: Traces in Category $\mathrm{A}_{2}$.

\begin{tabular}{|c|c|c|c|c|}
\hline${ }_{\beta \in \mathbb{F}_{2^{l}}} T_{i}(0+\beta)$ & $T_{1}(0+\beta)$ & $T_{2}(0+\beta)$ & $T_{3}(0+\beta)$ & $T_{4}(0+\beta)$ \\
\hline$\beta \in W_{0} \cap W_{4}$ & 0 & 0 & 0 & 0 \\
\hline$\beta \in W_{4} \backslash\left(W_{4} \cap W_{0}\right)$ & 0 & 0 & 0 & 1 \\
\hline$\beta \in W_{0} \backslash\left(W_{4} \cap W_{0}\right)$ & 0 & 0 & 0 & 1 \\
\hline$\beta \in \mathbb{F}_{2^{l}} \backslash\left(W_{4} \cup W_{0}\right)$ & 0 & 0 & 0 & $\overline{0}$ \\
\hline
\end{tabular}




\section{Category $B_{1}$.}

In this category, $W_{2}(\gamma)=W_{4}(\gamma)=W_{0}$, and $W_{3}(\gamma)=\mathbb{F}_{2^{l}}$. So we divide $\mathbb{F}_{2^{l}}$ into two subspaces, one is $W_{2}$, and the other is $\mathbb{F}_{2^{l}} \backslash W_{2}$. By Propositions 54 and 66, in each of these two subspaces we can find $T_{i}(\beta+\gamma)$, for $i=1,2,3,4$. We have Table 6.7 for the traces in the category $\mathrm{B}_{1}$.

Table 6.7: Traces in Category $B_{1}$.

\begin{tabular}{|c|c|c|c|c|}
\hline$\sum_{\beta \in \mathbb{F}_{2^{l}}} T_{i}(\beta+\gamma)$ & $T_{1}(\beta+\gamma)$ & $T_{2}(\beta+\gamma)$ & $T_{3}(\beta+\gamma)$ & $T_{4}(\beta+\gamma)$ \\
\hline$\beta \in W_{2}$ & $\overline{T_{1}(\gamma)}$ & $\overline{T_{2}(\gamma)}$ & $\overline{T_{3}(\gamma)}$ & $\bar{T}(\gamma)$ \\
\hline$\beta \in \mathbb{F}_{2^{l}} \backslash W_{2}$ & $T_{1}(\gamma)$ & $T_{2}(\gamma)+1$ & $T_{3}(\gamma)$ & $T_{4}(\gamma)$ \\
\hline
\end{tabular}

\section{Category $\mathrm{B}_{2}$.}

This category is defined with $\gamma \in \mathbb{F}_{2^{n}}$ such that $W_{2}(\gamma)=W_{0}, W_{3}(\gamma)=\mathbb{F}_{2^{l}}, W_{4}(\gamma) \neq$ $W_{0}$, and $W_{4}(\gamma) \neq \mathbb{F}_{2^{l}}$. This divides $\mathbb{F}_{2^{l}}$ into four subspaces, and we can find the traces of $\beta+\gamma$ in each of them. Table 6.8 has all the traces in the category $\mathrm{B}_{2}$.

Table 6.8: Traces in Category $\mathrm{B}_{2}$.

\begin{tabular}{|c|c|c|c|c|}
\hline$\sum_{\beta \in \mathbb{F}_{2^{l}}} T_{i}(\beta+\gamma)$ & $T_{1}(\beta+\gamma)$ & $T_{2}(\beta$ & $T_{3}(\beta+\gamma)$ & $\gamma)$ \\
\hline$\beta \in W_{4} \cap W_{0}$ & $T_{1}(\gamma)$ & $T_{2}(\gamma)$ & $T_{3}(\gamma)$ & $T_{4}(\gamma)$ \\
\hline$\beta \in W_{4}$ & 1 & $T_{2}(\gamma)+1$ & $I_{3}(\gamma)$ & $T_{4}(\gamma)+1$ \\
\hline$\beta \in W_{0} \backslash\left(W_{4} \cap W_{0}\right)$ & $T_{1}(\gamma)$ & $T_{2}(\gamma)$ & $T_{3}(\gamma)$ & $T_{4}(\gamma)+1$ \\
\hline$\beta \in \mathbb{F}_{2^{l}} \backslash\left(W_{4} \cup W_{0}\right)$ & $T_{1}(\gamma)$ & $T_{2}(\gamma)+1$ & $T_{3}(\gamma)$ & $T_{4}(\gamma)$ \\
\hline
\end{tabular}

\section{Category $\mathrm{C}_{1}$.}

For this category $\gamma \in \mathbb{F}_{2^{n}}$ is such that $W_{2}(\gamma)=W_{3}(\gamma)=W_{4}(\gamma)=W_{0}$. Then each $\beta \in \mathbb{F}_{2^{l}}$ can be in the subspace $W_{0}$, or the subspace $\mathbb{F}_{2^{l}} \backslash W_{0}$. Therefore we have Table 6.9 for the traces in the category $\mathrm{C}_{1}$.

\section{Category $\mathrm{C}_{2}$.}

This category has those cosets defined by $\gamma \in \mathbb{F}_{2^{n}}$ such that $W_{4}(\gamma) \neq W_{0}, W_{4}(\gamma) \neq \mathbb{F}_{2^{l}}$, and $W_{2}(\gamma)=W_{3}(\gamma)=W_{0}$. Then each $\beta \in \mathbb{F}_{2^{l}}$ is in four different subspaces, and in 
Table 6.9: Traces in Category $\mathrm{C}_{1}$.

\begin{tabular}{|c|c|c|c|c|}
\hline $\bar{\beta}_{\beta \in \mathbb{F}_{2^{l}}} \bar{T}_{i}(\beta+\gamma)$ & $T_{1}(\beta+\gamma)$ & $T_{2}(\beta+\gamma)$ & $T_{3}(\beta+\gamma)$ & $T_{4}(\beta+\gamma)$ \\
\hline$\beta \in W_{0}$ & $T_{1}(\gamma)$ & $T_{2}(\gamma)$ & $T_{3}(\gamma)$ & $T_{4}(\gamma)$ \\
\hline$\beta \in \mathbb{F}_{2^{l}} \backslash W_{0}$ & $T_{1}(\gamma)$ & $T_{2}(\gamma)+1$ & $T_{3}(\gamma)+1$ & $T_{4}(\gamma)$ \\
\hline
\end{tabular}

each of these subspaces we can compute the traces of $\beta+\gamma$. Table 6.10 has all the traces for the category $\mathrm{C}_{2}$.

Table 6.10: Traces in Category $\mathrm{C}_{2}$.

\begin{tabular}{|c|c|c|c|c|}
\hline${ }_{\beta \in \mathbb{F}_{2^{l}}} T_{i}(\beta+\gamma)$ & $T_{1}(\beta+\gamma)$ & $T_{2}(\beta+\gamma)$ & $T_{3}(\beta+\gamma)$ & $T_{4}(\beta+\gamma)$ \\
\hline$\beta \in W_{4} \cap W_{0}$ & $T_{1}(\gamma)$ & $T_{2}(\gamma)$ & $T_{3}(\gamma)$ & $T_{4}(\gamma)$ \\
\hline$\beta \in W_{4} \backslash\left(W_{4} \cap W_{0}\right)$ & $T_{1}(\gamma)$ & $T_{2}(\gamma)+1$ & $T_{3}(\gamma)+1$ & $T_{4}(\gamma)+1$ \\
\hline$\beta \in W_{0} \backslash\left(W_{4} \cap W_{0}\right)$ & $T_{1}(\gamma)$ & $T_{2}(\gamma)$ & $T_{3}(\gamma)$ & $T_{4}(\gamma)+1$ \\
\hline$\beta \in \mathbb{F}_{2^{l}} \backslash\left(W_{4} \cup W_{0}\right)$ & $T_{1}(\gamma)$ & $T_{2}(\gamma)+1$ & $T_{3}(\gamma)+1$ & $T_{4}(\gamma)$ \\
\hline
\end{tabular}

\section{Category $D_{1}$.}

In this category, $W_{2}(\gamma) \neq W_{0}, W_{3}(\gamma)=W_{4}(\gamma)=W_{0}$. In Table 6.11 we have different traces in the category $D_{1}$.

Table 6.11: Traces in Category $\mathrm{D}_{1}$.

\begin{tabular}{|c|c|c|c|c|}
\hline${ }_{\beta \in \mathbb{F}_{2^{l}}} T_{i}(\beta+\gamma)$ & $T_{1}(\beta+\gamma)$ & $T_{2}(\beta+\gamma)$ & $T_{3}(\beta+\gamma)$ & $T_{4}(\beta+\gamma)$ \\
\hline$\beta \in W_{2} \cap W_{0}$ & $T_{1}(\gamma)$ & $T_{2}(\gamma)$ & $T_{3}(\gamma)$ & $T_{4}(\gamma)$ \\
\hline$\beta \in W_{2} \backslash\left(W_{2} \cap W_{0}\right)$ & $\overline{T_{1}(\gamma)}$ & $T_{2}(\gamma)$ & $T_{3}(\gamma)+1$ & $\overline{T_{4}(\gamma)}$ \\
\hline$\beta \in W_{0} \backslash\left(W_{2} \cap W_{0}\right)$ & $T_{1}(\gamma)$ & $T_{2}(\gamma)+1$ & $T_{3}(\gamma)$ & $T_{4}(\gamma)$ \\
\hline$\beta \in \mathbb{F}_{2^{l}} \backslash\left(W_{2} \cup W_{0}\right)$ & $T_{1}(\gamma)$ & $T_{2}(\gamma)+1$ & $T_{3}(\gamma)+1$ & $T_{4}(\gamma)$ \\
\hline
\end{tabular}

\section{Category $\mathrm{D}_{2}$.}

In this category, $W_{2}(\gamma) \neq W_{0}, W_{3}(\gamma)=W_{0}$, and $W_{4}(\gamma) \neq W_{0}$ and $W_{4}(\gamma) \neq \mathbb{F}_{2^{l}}$.

Table 6.12 has different traces of the category $\mathrm{D}_{2}$.

Category $\mathrm{E}_{\mathbf{1}}$.

In this category, $W_{2}(\gamma) \neq W_{0}, W_{3}(\gamma) \neq W_{0}$, and $W_{4}(\gamma)=W_{0}$. For the traces in the category $\mathrm{E}_{1}$ we have Table 6.13. 
Table 6.12: Traces in Category $\mathrm{D}_{2}$.

\begin{tabular}{|c|c|c|c|c|}
\hline$T_{i}(\beta+\gamma)$ & $T_{1}(\beta+\gamma)$ & $T_{2}(\beta+\gamma)$ & $T_{3}(\beta+\gamma)$ & $T_{4}(\beta+\gamma)$ \\
\hline$\beta \in \mathbb{F}_{2^{l}}$ & & & \\
\hline$\beta \in W_{2} \cap W_{3} \cap W_{4}$ & $T_{1}(\gamma)$ & $T_{2}(\gamma)$ & $T_{3}(\gamma)$ & $T_{4}(\gamma)$ \\
\hline$\beta \in\left(W_{2} \cap W_{3}\right) \backslash W_{4}$ & $T_{1}(\gamma)$ & $T_{2}(\gamma)$ & $T_{3}(\gamma)$ & $T_{4}(\gamma)+1$ \\
\hline$\beta \in\left(W_{2} \cap W_{4}\right) \backslash W_{3}$ & $T_{1}(\gamma)$ & $T_{2}(\gamma)$ & $T_{3}(\gamma)+1$ & $T_{4}(\gamma)+1$ \\
\hline$\beta \in\left(W_{3} \cap W_{4}\right) \backslash W_{2}$ & $T_{1}(\gamma)$ & $T_{2}(\gamma)+1$ & $T_{3}(\gamma)$ & $T_{4}(\gamma)$ \\
\hline$\beta \in W_{4} \backslash\left(W_{2} \cup W_{3}\right)$ & $T_{1}(\gamma)$ & $T_{2}(\gamma)+1$ & $T_{3}(\gamma)+1$ & $T_{4}(\gamma)+1$ \\
\hline$\beta \in W_{3} \backslash\left(W_{2} \cup W_{4}\right)$ & $T_{1}(\gamma)$ & $T_{2}(\gamma)+1$ & $T_{3}(\gamma)$ & $T_{4}(\gamma)+1$ \\
\hline$\beta \in W_{2} \backslash\left(W_{3} \cup W_{4}\right)$ & $T_{1}(\gamma)$ & $T_{2}(\gamma)$ & $T_{3}(\gamma)+1$ & $T_{4}(\gamma)$ \\
\hline$\beta \in \mathbb{F}_{2^{l}} \backslash\left(W_{2} \cup W_{3} \cup W_{4}\right)$ & $T_{1}(\gamma)$ & $T_{2}(\gamma)+1$ & $T_{3}(\gamma)+1$ & $T_{4}(\gamma)$ \\
\hline
\end{tabular}

Table 6.13: Traces in Category $\mathrm{E}_{1}$.

\begin{tabular}{|c|c|c|c|c|}
\hline$\underbrace{}_{\beta \in \mathbb{F}_{2^{l}}} T_{i}(\beta+\gamma)$ & $T_{1}(\beta+\gamma)$ & $T_{2}(\beta+\gamma)$ & $T_{3}(\beta+\gamma)$ & $T_{4}(\beta+\gamma)$ \\
\hline$\beta \in W_{2} \cap W_{3} \cap W_{4}$ & $T_{1}(\gamma)$ & $T_{2}(\gamma)$ & $T_{3}(\gamma)$ & $T_{4}(\gamma)$ \\
\hline$\beta \in\left(W_{2} \cap W_{3}\right) \backslash W_{4}$ & $T_{1}(\gamma)$ & $T_{2}(\gamma)$ & $T_{3}(\gamma)$ & $T_{4}(\gamma)$ \\
\hline$\beta \in\left(W_{2} \cap W_{4}\right) \backslash W_{3}$ & $T_{1}(\gamma)$ & $T_{2}(\gamma)$ & $T_{3}(\gamma)+1$ & $T_{4}(\gamma)$ \\
\hline$\beta \in\left(W_{3} \cap W_{4}\right) \backslash W_{2}$ & $T_{1}(\gamma)$ & $T_{2}(\gamma)+1$ & $T_{3}(\gamma)$ & $T_{4}(\gamma)$ \\
\hline$\beta \in W_{4} \backslash\left(W_{2} \cup W_{3}\right)$ & $T_{1}(\gamma)$ & $T_{2}(\gamma)+1$ & $T_{3}(\gamma)+1$ & $T_{4}(\gamma)$ \\
\hline$\beta \in W_{3} \backslash\left(W_{2} \cup W_{4}\right)$ & $T_{1}(\gamma)$ & $T_{2}(\gamma)+1$ & $T_{3}(\gamma)$ & $T_{4}(\gamma)$ \\
\hline$\beta \in W_{2} \backslash\left(W_{3} \cup W_{4}\right)$ & $T_{1}(\gamma)$ & $T_{2}(\gamma)$ & $T_{3}(\gamma)+1$ & $T_{4}(\gamma)$ \\
\hline$\beta \in \mathbb{F}_{2^{l}} \backslash\left(W_{2} \cup W_{3} \cup W_{4}\right)$ & $T_{1}(\gamma)$ & $T_{2}(\gamma)+1$ & $T_{3}(\gamma)+1$ & $T_{4}(\gamma)$ \\
\hline
\end{tabular}

\section{Category $\mathbf{E}_{\mathbf{2}}$.}

In this category, $W_{i}(\gamma), i=2,3,4$ are hyperplanes different from $W_{0}$. All the traces of a given element $\beta+\gamma$ of this category are given in Table 6.14.

\section{Remark: what remains to be done.}

Each category of cosets is recognized with some condition(s) on $\gamma \in \mathbb{F}_{2^{n}}$. As an example in category $\mathrm{A}$, we have cosets $\gamma+\mathbb{F}_{2^{l}}$ such that $W_{i}(\gamma)=\mathbb{F}_{2^{l}}$, where $1 \leq i \leq 4$, and the conditions on $\gamma$ are given in Proposition 60.

For $\beta \in \mathbb{F}_{2^{l}}$ and $i=1, \ldots, 4$, we computed different traces $T_{i}(\gamma+\beta)$. These are given in terms of $T_{i}(\gamma)$, that are either zero or one. We expect that $T_{i}(\gamma)$ depend on $m$, or $l$, where $n=2 m=4 l$. For each condition on $\gamma$ related to a given category, 
Table 6.14: Traces in Category $\mathrm{E}_{2}$.

\begin{tabular}{|c|c|c|c|c|}
\hline$T_{i}(\beta+\gamma)$ & $T_{1}(\beta+\gamma)$ & $T_{2}(\beta+\gamma)$ & $T_{3}(\beta+\gamma)$ & $T_{4}(\beta+\gamma)$ \\
\hline$\beta \in W_{2} \cap W_{2} \cap W_{3} \cap W_{4}$ & $T_{1}(\gamma)$ & $T_{2}(\gamma)$ & $T_{3}(\gamma)$ & $T_{4}(\gamma)$ \\
\hline$\beta \in\left(W_{0} \cap W_{2} \cap W_{3}\right) \backslash W_{4}$ & $T_{1}(\gamma)$ & $T_{2}(\gamma)$ & $T_{3}(\gamma)$ & $T_{4}(\gamma)+1$ \\
\hline$\beta \in\left(W_{0} \cap W_{2} \cap W_{4}\right) \backslash W_{3}$ & $T_{1}(\gamma)$ & $T_{2}(\gamma)$ & $T_{3}(\gamma)+1$ & $T_{4}(\gamma)$ \\
\hline$\beta \in\left(W_{0} \cap W_{3} \cap W_{4}\right) \backslash W_{2}$ & $T_{1}(\gamma)$ & $T_{2}(\gamma)+1$ & $T_{3}(\gamma)$ & $T_{4}(\gamma)$ \\
\hline$\beta \in\left(W_{2} \cap W_{3} \cap W_{4}\right) \backslash W_{0}$ & $T_{1}(\gamma)$ & $T_{2}(\gamma)$ & $T_{3}(\gamma)$ & $T_{4}(\gamma)+1$ \\
\hline$\beta \in\left(W_{0} \cap W_{2}\right) \backslash\left(W_{3} \cup W_{4}\right)$ & $T_{1}(\gamma)$ & $T_{2}(\gamma)$ & $T_{3}(\gamma)+1$ & $T_{4}(\gamma)+1$ \\
\hline$\beta \in\left(W_{0} \cap W_{3}\right) \backslash\left(W_{2} \cup W_{4}\right)$ & $T_{1}(\gamma)$ & $T_{2}(\gamma)+1$ & $T_{3}(\gamma)$ & $T_{4}(\gamma)+1$ \\
\hline$\beta \in\left(W_{0} \cap W_{4}\right) \backslash\left(W_{2} \cup W_{3}\right)$ & $T_{1}(\gamma)$ & $T_{2}(\gamma)+1$ & $T_{3}(\gamma)+1$ & $T_{4}(\gamma)$ \\
\hline$\beta \in\left(W_{2} \cap W_{3}\right) \backslash\left(W_{0} \cup W_{4}\right)$ & $T_{1}(\gamma)$ & $T_{2}(\gamma)$ & $T_{3}(\gamma)$ & $T_{4}(\gamma)$ \\
\hline$\beta \in\left(W_{2} \cap W_{4}\right) \backslash\left(W_{0} \cup W_{3}\right)$ & $T_{1}(\gamma)$ & $T_{2}(\gamma)$ & $T_{3}(\gamma)+1$ & $T_{4}(\gamma)+1$ \\
\hline$\beta \in\left(W_{3} \cap W_{4}\right) \backslash\left(W_{0} \cup W_{2}\right)$ & $T_{1}(\gamma)$ & $T_{2}(\gamma)+1$ & $T_{3}(\gamma)$ & $T_{4}(\gamma)+1$ \\
\hline$\beta \in W_{0} \backslash\left(W_{2} \cup W_{3} \cup W_{4}\right)$ & $T_{1}(\gamma)$ & $T_{2}(\gamma)+1$ & $T_{3}(\gamma)+1$ & $T_{4}(\gamma)+1$ \\
\hline$\beta \in W_{2} \backslash\left(W_{0} \cup W_{3} \cup W_{4}\right)$ & $T_{1}(\gamma)$ & $T_{2}(\gamma)$ & $T_{3}(\gamma)+1$ & $T_{4}(\gamma)$ \\
\hline$\beta \in W_{3} \backslash\left(W_{0} \cup W_{2} \cup W_{4}\right)$ & $T_{1}(\gamma)$ & $T_{2}(\gamma)+1$ & $T_{3}(\gamma)$ & $T_{4}(\gamma)$ \\
\hline$\beta \in W_{4} \backslash\left(W_{0} \cup W_{2} \cup W_{3}\right)$ & $T_{1}(\gamma)$ & $T_{2}(\gamma)+1$ & $T_{3}(\gamma)+1$ & $T_{4}(\gamma)+1$ \\
\hline$\beta \in \mathbb{F}_{2^{l}} \backslash\left(W_{0} \cup W_{2} \cup W_{3} \cup W_{4}\right)$ & $T_{1}(\gamma)$ & $T_{2}(\gamma)+1$ & $T_{3}(\gamma)+1$ & $T_{4}(\gamma)$ \\
\hline
\end{tabular}

we compute $T_{i}(\gamma)$. Then the computational results obtained by Maple codes show that in each of the categories, the traces $T_{i}$ are evenly zero and one. It is not clear under what condition(s) on $m$ or $l$ they are zero or one. Once these conditions are obtained we should be able to compute the exact result for $F\left(n, t_{1}, \ldots, t_{4}\right)$, where $n=4 l$. In $[24]$ it is conjectured that

$$
F\left(n, t_{1}, \ldots, t_{r}\right)=2^{n-r}+G\left(n, t_{1}, \ldots, t_{r}\right),
$$

and $G\left(n, t_{1}, \ldots, t_{r}\right)$ is in terms of different powers of 2 . In our case, we have

$$
F\left(n, t_{1}, \ldots, t_{4}\right)=2^{n-4}+G\left(n, t_{1}, \ldots, t_{4}\right),
$$

and finding the exact value of $F\left(n, t_{1}, \ldots, t_{4}\right)$ means finding the powers of 2 in $G\left(n, t_{1}, \ldots, t_{4}\right)$. 
Similar results to the studies in this chapter are then needed for the case $n=4 l+2$.

Later in Chapter 7 we find an approximation for the number $F\left(n, t_{1}, t_{2}, t_{3}, t_{4}\right)$ that can be used to compute $N\left(n, t_{1}, t_{2}, t_{3}, t_{4}\right)$. 


\title{
Chapter 7
}

\section{Approximating the Number}

\author{
$N\left(n, t_{1}, t_{2}, t_{3}, t_{4}\right)$
}

In this chapter we give an approximation for the number $N\left(n, t_{1}, t_{2}, t_{3}, t_{4}\right)$. In Section 7.1 we give an estimate for the number $F\left(n, t_{1}, t_{2}, t_{3}, t_{4}\right)$. Then in Section 7.2 we use this estimation to find an approximation for $N\left(n, t_{1}, t_{2}, t_{3}, t_{4}\right)$. Finally in Section 7.3 for different values of $n$ we compute this approximation, and compare it with the exact value of $N\left(n, t_{1}, t_{2}, t_{3}, t_{4}\right)$. This shows that our approximation is a very good estimate for the number $N\left(n, t_{1}, t_{2}, t_{3}, t_{4}\right)$.

\subsection{Estimation of $F\left(n, t_{1}, t_{2}, t_{3}, t_{4}\right)$}

For different values of $t_{i} \in \mathbb{F}_{2}$, where $i=1, \ldots, 4$, we have 16 cases for $\left(t_{1}, t_{2}, t_{3}, t_{4}\right)$. In Theorem 50 these 16 cases are divided into 8 groups, and for different groups the formulas for the number $N\left(n, t_{1}, t_{2}, t_{3}, t_{4}\right)$ are given (see Theorem 50). Each group has some of the cases of $\left(t_{1}, t_{2}, t_{3}, t_{4}\right)$ that are connected to each other. For example, 
in group $(i)$ we have the cases

$$
(1,1,1,0),(1,0,0,1),(1,1,1,1),(1,0,0,0) \text {. }
$$

In groups (ii) and (iii) we have the cases $(0,0,1,0)$ and $(0,0,1,1)$, respectively. For the first 12 cases of $\left(t_{1}, t_{2}, t_{3}, t_{4}\right)$ which are in groups $(i)-(v)$ in Theorem 50, the formulas for $N\left(n, t_{1}, t_{2}, t_{3}, t_{4}\right)$ are in terms of $F\left(n / d, t_{1}^{\prime}, t_{2}^{\prime}, t_{3}^{\prime}, t_{4}^{\prime}\right)$ 's, where $d$ is an odd divisor of $n$ congruent 8 , and $\left(t_{1}^{\prime}, t_{2}^{\prime}, t_{3}^{\prime}, t_{4}^{\prime}\right)$ are in the same group as $\left(t_{1}, t_{2}, t_{3}, t_{4}\right)$. In the last 4 cases, the values of $N\left(n, t_{1}, t_{2}, t_{3}, t_{4}\right)$ are given using $F\left(n / d, t_{1}^{\prime}, t_{2}^{\prime}, t_{3}^{\prime}, t_{4}^{\prime}\right)$ and $F\left(n / 2 d, t_{1}^{\prime}, t_{2}^{\prime}\right)$, where $d$ is an odd divisor of $n$ congruent 4 , and $\left(t_{1}^{\prime}, t_{2}^{\prime}, t_{3}^{\prime}, t_{4}^{\prime}\right)$ is from the same group as $\left(t_{1}, t_{2}, t_{3}, t_{4}\right)$. When two coefficients are prescribed then by [3] we have $F\left(n, t_{1}, t_{2}\right)=2^{n-2}+G\left(n, t_{1}, t_{2}\right)$, where $G\left(n, t_{1}, t_{2}\right)$ are given in Table 5.1. If $n$ is even, then in [24] the number $F\left(n, t_{1}, t_{2}, t_{3}\right)$ is given as

$$
F\left(n, t_{1}, t_{2}, t_{3}\right)=2^{n-3}+G\left(n, t_{1}, t_{2}, t_{3}\right),
$$

where $G\left(n, t_{1}, t_{2}, t_{3}\right)$ can be computed using the Tables 5.2 and 5.3.

Let $n \geq 4$ be an even number. In each of the 16 cases of Theorem 50 we use an estimate of $F\left(n / d, t_{1}^{\prime}, t_{2}^{\prime}, t_{3}^{\prime}, t_{4}^{\prime}\right)$ to approximate $N\left(n, t_{1}, t_{2}, t_{3}, t_{4}\right)$. We let

$$
F\left(n / d, t_{1}^{\prime}, t_{2}^{\prime}, t_{3}^{\prime}, t_{4}^{\prime}\right) \approx \begin{cases}2^{n / d-4} & \text { if } n / d \geq 4 \\ 0 & \text { otherwise }\end{cases}
$$

or $F\left(n / d, t_{1}^{\prime}, t_{2}^{\prime}, t_{3}^{\prime}, t_{4}^{\prime}\right) \approx\left[n / p_{1} \geq 4\right] 2^{n / d-4}$ where again $[P]=1$, if the statement $P$ is true; otherwise $[P]=0$. Therefore, $F\left(n, t_{1}, t_{2}, t_{3}, t_{4}\right) \approx 2^{n-4}$, since we have $n \geq 4$. 


\subsection{An Approximation of $N\left(n, t_{1}, t_{2}, t_{3}, t_{4}\right)$}

In this section, we consider different cases for the even number $n$. Then in each case for $n$, we choose $\left(t_{1}, t_{2}, t_{3}, t_{4}\right)$ from each of the 8 groups in Theorem 50 , and we use the estimate for $F\left(n, t_{1}, t_{2}, t_{3}, t_{4}\right)$ and the exact value of $F\left(n / d, t_{1}, t_{2}\right)$, given in Table 5.1, to find an approximation of $N\left(n, t_{1}, t_{2}, t_{3}, t_{4}\right)$.

To understand the computations, we consider 4 cases $n=2^{k_{0}}, 2^{k_{0}} p_{1}{ }^{k_{1}}, 2^{k_{0}} p_{1}{ }^{k_{1}} p_{2}{ }^{k_{2}}$, and finally, in general case, $n=2^{k_{0}} p_{1}^{k_{1}} \cdots p_{k}{ }^{k_{s}}$, where $k_{0}, \ldots, k_{s} \geq 1$.

Case 1: $n=2^{k_{0}}$.

In the simplest case, let $n=2^{k_{0}}$, where $k_{0} \geq 3$, (since $k_{0}=1$, or 2 gives a small $n$ we assume $k_{0} \geq 3$ ). Then the only odd divisor of $n$ congruent 8 (and 4) is $d=1$. This implies that in the first 12 cases of Theorem 50, we just have one sum in the right side of each of the equations. Thus in those 12 cases, for any $\left(t_{1}, t_{2}, t_{3}, t_{4}\right)$, we have

$$
n N\left(n, t_{1}, t_{2}, t_{3}, t_{4}\right)=F\left(n, t_{1}, t_{2}, t_{3}, t_{4}\right) \approx 2^{n-4} .
$$

Therefore,

$$
N\left(n, t_{1}, t_{2}, t_{3}, t_{4}\right) \approx \frac{2^{n-4}}{n}=\frac{2^{n-4}}{2^{k_{0}}}=2^{n-k_{0}-4} .
$$

Let us compute $N\left(n, t_{1}, t_{2}, t_{3}, t_{4}\right)$ in the last four cases of $\left(t_{1}, t_{2}, t_{3}, t_{4}\right)$, which are in groups (vi), (vii) and (viii) of Theorem 50. By Theorem 50 and our approximation we have

$$
\begin{aligned}
& n N(n, 0,0,0,0)=F(n, 0,0,0,0)-F(n / 2,0,0) \approx 2^{n-4}-F(n / 2,0,0), \\
& n N(n, 0,0,0,1)=F(n, 0,0,0,1)-F(n / 2,0,1) \approx 2^{n-4}-F(n / 2,0,1), \\
& n N(n, 0,1,0,0)=F(n, 0,1,0,0)-F(n / 2,0,0) \approx 2^{n-4}-F(n / 2,1,0),
\end{aligned}
$$




$$
n N(n, 0,1,0,1)=F(n, 0,1,0,1)-F(n / 2,0,0) \approx 2^{n-4}-F(n / 2,1,1),
$$

where for different $t_{1}^{\prime}, t_{2}^{\prime} \in \mathbb{F}_{2}$ the numbers $F\left(n / 2, t_{1}^{\prime}, t_{2}^{\prime}\right)$ can be obtained by Table 5.1. We have $n=2^{k_{0}}$ and $k_{0} \geq 3$. Then $m=\frac{n}{2}=2^{k_{0}-1}$, and $m \equiv 0(\bmod 4)$. Thus by Table 5.1 we have

$$
\begin{aligned}
& F(n / 2,0,0)=2^{n / 2-2}-2^{n / 4-1}, \\
& F(n / 2,0,1)=2^{n / 2-2}+2^{n / 4-1}, \\
& F(n / 2,1,0)=F(n / 2,1,1)=2^{n / 2-2} .
\end{aligned}
$$

Let $G=\{(i), \ldots,(v)\}$ be the set of groups of the first 12 cases for $\left(t_{1}, t_{2}, t_{3}, t_{4}\right)$ given in Theorem 50. Then briefly for $n=2^{k_{0}}$ where $k_{0} \geq 3$ an estimate of $N\left(n, t_{1}, t_{2}, t_{3}, t_{4}\right)$ can be given by

$$
N\left(n, t_{1}, t_{2}, t_{3}, t_{4}\right) \approx \begin{cases}2^{n-k_{0}-4} & \text { if }\left(t_{1}, t_{2}, t_{3}, t_{4}\right) \in G, \\ \left(2^{n-4}-2^{n / 2-2}+2^{n / 4-1}\right) / n & \text { if }\left(t_{1}, t_{2}, t_{3}, t_{4}\right)=(0,0,0,0), \\ \left(2^{n-4}-2^{n / 2-2}-2^{n / 4-1}\right) / n & \text { if }\left(t_{1}, t_{2}, t_{3}, t_{4}\right)=(0,0,0,1), \\ \left(2^{n-4}-2^{n / 2-2}\right) / n & \text { if }\left(t_{1}, t_{2}, t_{3}, t_{4}\right) \text { in group (viii) }\end{cases}
$$

Case 2: $n=2^{k_{0}} p_{1}^{k_{1}}$.

Next, let $n=2^{k_{0}} p_{1}^{k_{1}}$ where $k_{0}, k_{1} \geq 1$. Then 1 and $p_{1}$ are the only odd divisors of $n$ with $\mu\left(p_{1}\right)=-1$. We define $S_{1}=\left\{p_{1}\right\}$. Note that if $d=p_{1}{ }^{k_{1}{ }^{\prime}}$ such that $2 \leq k_{1}^{\prime} \leq k_{1}$, then $p_{1}^{2} \mid d$, and therefore $\mu(d)=0$. Then the related term in $N\left(n, t_{1}, t_{2}, t_{3}, t_{4}\right)$ vanishes. Let us define $S=\{1,3,5,7\}$. Then for $d=p_{1}$ we have $\mu(d)=-1$, and

$$
d \equiv k \quad(\bmod 8) \text { for some } k \in S .
$$


Also $d \equiv k(\bmod 4)$ for some $k^{\prime} \in S^{\prime}=\{1,3\}$. To estimate $N\left(n, t_{1}, t_{2}, t_{3}, t_{4}\right)$, first let $\left(t_{1}, t_{2}, t_{3}, t_{4}\right)$ be in group $(i)$. Then by Theorem $50(i)$ we have

$$
\begin{aligned}
n N\left(n, t_{1}, t_{2}, t_{3}, t_{4}\right) & =F\left(n, t_{1}, t_{2}, t_{3}, t_{4}\right)-F\left(n / p_{1}, t_{1}^{\prime}, t_{2}^{\prime}, t_{3}^{\prime}, t_{4}^{\prime}\right) \\
& \approx 2^{n-4}-\left[n / p_{1} \geq 4\right] 2^{n / p_{1}-4},
\end{aligned}
$$

where $\left(t_{1}^{\prime}, t_{2}^{\prime}, t_{3}^{\prime}, t_{4}^{\prime}\right)$ is in group $(i)$. Thus

$$
N\left(n, t_{1}, t_{2}, t_{3}, t_{4}\right) \approx \frac{1}{n}\left(2^{n-4}-\left[n / p_{1} \geq 4\right] 2^{n / p_{1}-4}\right) .
$$

If $\left(t_{1}, t_{2}, t_{3}, t_{4}\right)$ is in group $(i i)$, then $\left(t_{1}, t_{2}, t_{3}, t_{4}\right)=(0,0,1,0)$ and we have

$$
\begin{aligned}
n N(n, 0,0,1,0) & =F(n, 0,0,1,0)-F\left(n / p_{1}, 0,0,1,0\right) \\
& \approx 2^{n-4}-\left[n / p_{1} \geq 4\right] 2^{n / p_{1}-4},
\end{aligned}
$$

or

$$
N(n, 0,0,1,0) \approx \frac{1}{n}\left(2^{n-4}-\left[n / p_{1} \geq 4\right] 2^{n / p_{1}-4}\right) .
$$

Similar to group (ii), if $\left(t_{1}, t_{2}, t_{3}, t_{4}\right)$ is in group (iii), then

$$
\begin{aligned}
N(n, 0,0,1,1) & =\frac{1}{n}\left(F(n, 0,0,1,1)-F\left(n / p_{1}, 0,0,1,0\right)\right) \\
& \approx \frac{1}{n}\left(2^{n-4}-\left[n / p_{1} \geq 4\right] 2^{n / p_{1}-4}\right) .
\end{aligned}
$$

If $\left(t_{1}, t_{2}, t_{3}, t_{4}\right)$ is in group $(i v)$, then with an argument similar to the case $\left(t_{1}, t_{2}, t_{3}, t_{4}\right)$ in group $(i)$ we have

$$
N\left(n, t_{1}, t_{2}, t_{3}, t_{4}\right) \approx \frac{1}{n}\left(2^{n-4}-\left[n / p_{1} \geq 4\right] 2^{n / p_{1}-4}\right)
$$


Assume that $\left(t_{1}, t_{2}, t_{3}, t_{4}\right)$ is in group $(v)$, which implies $\left(t_{1}, t_{2}, t_{3}, t_{4}\right)=(0,1,1,1)$, or $(0,1,1,0)$. It is clear that $p_{1} \equiv 1$, or $3(\bmod 4)$, and $\mu\left(p_{1}\right)=-1, \mu\left(p_{1}{ }^{k}\right)=0$, for $k>1$. By Theorem $50(v)$ if $p_{1} \equiv 1(\bmod 4)$ then we have

$$
\begin{aligned}
n N(n, 0,1,1,1) & =F(n, 0,1,1,1)-F\left(n / p_{1}, 0,1,1,1\right) \\
& \approx 2^{n-4}-\left[n / p_{1} \geq 4\right] 2^{n / p_{1}-4}, \\
n N(n, 0,1,1,0) & =F(n, 0,1,1,0)-F\left(n / p_{1}, 0,1,1,0\right) \\
& \approx 2^{n-4}-\left[n / p_{1} \geq 4\right] 2^{n / p_{1}-4} .
\end{aligned}
$$

Otherwise, when $p_{1} \equiv 3(\bmod 4)$ we have

$$
\begin{aligned}
n N(n, 0,1,1,1) & =F(n, 0,1,1,1)-F\left(n / p_{1}, 0,1,1,0\right) \\
& \approx 2^{n-4}-\left[n / p_{1} \geq 4\right] 2^{n / p_{1}-4}, \\
n N(n, 0,1,1,0) & =F(n, 0,1,1,0)-F\left(n / p_{1}, 0,1,1,1\right) \\
& \approx 2^{n-4}-\left[n / p_{1} \geq 4\right] 2^{n / p_{1}-4} .
\end{aligned}
$$

Therefore in this group we have

$$
N(n, 0,1,1,1)=N(n, 0,1,1,0) \approx \frac{1}{n}\left(2^{n-4}-\left[n / p_{1} \geq 4\right] 2^{n / p_{1}-4}\right) .
$$

Let us compute $N\left(n, t_{1}, t_{2}, t_{3}, t_{4}\right)$, when $\left(t_{1}, t_{2}, t_{3}, t_{4}\right)$ is from the groups (vi), (vii) and (viii). In group (vi) we have

$$
\begin{aligned}
n N(n, 0,0,0,0) & =F(n, 0,0,0,0)-F\left(n / p_{1}, 0,0,0,0\right) \\
& -F(n / 2,0,0)+F\left(n / 2 p_{1}, 0,0\right) \\
& \approx 2^{n-4}-\left[n / p_{1} \geq 4\right] 2^{n / p_{1}-4}-F(n / 2,0,0)+F\left(n / 2 p_{1}, 0,0\right) .
\end{aligned}
$$


Thus

$$
N(n, 0,0,0,0) \approx \frac{1}{n}\left(2^{n-4}-\left[n / p_{1} \geq 4\right] 2^{n / p_{1}-4}-F(n / 2,0,0)+F\left(n / 2 p_{1}, 0,0\right)\right)
$$

where for a given $n$, the numbers $F(n / 2,0,0)$ and $F\left(n / 2 p_{1}, 0,0\right)$ can be computed using Table 5.1. Similarly in group (vii) we have

$$
N(n, 0,0,0,1) \approx \frac{1}{n}\left(2^{n-4}-\left[n / p_{1} \geq 4\right] 2^{n / p_{1}-4}-F(n / 2,0,1)+F\left(n / 2 p_{1}, 0,1\right)\right)
$$

Finally let $\left(t_{1}, t_{2}, t_{3}, t_{4}\right)$ be in the group (viii). Then by Theorem $50(v i i i)$ we have

$$
\begin{aligned}
n N(n, 0,1,0,0) & =F(n, 0,1,0,0)-F(n / 2,1,0) \\
& -\left[p_{1} \equiv 1\right]\left(F\left(n / 2 p_{1}, 0,1,0,0\right)-F\left(n / 2 p_{1}, 1,0\right)\right) \\
& -\left[p_{1} \equiv 3\right]\left(F\left(n / 2 p_{1}, 0,1,0,1\right)-F\left(n / 2 p_{1}, 1,1\right)\right) \\
& \approx 2^{n-4}-F(n / 2,1,0) \\
& -\left[p_{1} \equiv 1\right]\left[n / p_{1} \geq 4\right] 2^{n / p_{1}-4}-\left[p_{1} \equiv 3\right]\left[n / p_{1} \geq 4\right] 2^{n / p_{1}-4} \\
& +\left[p_{1} \equiv 1\right] F\left(n / 2 p_{1}, 1,0\right)+\left[p_{1} \equiv 3\right] F\left(n / 2 p_{1}, 1,1\right) .
\end{aligned}
$$

For $d=p_{1}$ either $p_{1} \equiv 1(\bmod 4)$, or $p_{1} \equiv 3(\bmod 4)$. Thus

$$
\begin{aligned}
N(n, 0,1,0,0) & \approx \frac{1}{n}\left(2^{n-4}-\left[n / p_{1} \geq 4\right] 2^{n / p_{1}-4}-F(n / 2,1,0)\right) \\
& +\frac{1}{n}\left(\left[p_{1} \equiv 1\right] F\left(n / 2 p_{1}, 1,0\right)+\left[p_{1} \equiv 3\right] F\left(n / 2 p_{1}, 1,1\right)\right) .
\end{aligned}
$$


Also for $\left(t_{1}, t_{2}, t_{3}, t_{4}\right)=(0,1,0,1)$ in group (viii) we have

$$
\begin{aligned}
N(n, 0,1,0,1) & \approx \frac{1}{n}\left(2^{n-4}-\left[n / p_{1} \geq 4\right] 2^{n / p_{1}-4}-F(n / 2,1,1)\right) \\
& +\frac{1}{n}\left(\left[p_{1} \equiv 1\right] F\left(n / 2 p_{1}, 1,1\right)+\left[p_{1} \equiv 3\right] F\left(n / 2 p_{1}, 1,0\right)\right) .
\end{aligned}
$$

Briefly, if $n=2^{k_{0}} p_{1}^{k_{1}}$, for the first 12 cases of $\left(t_{1}, t_{2}, t_{3}, t_{4}\right)$ we have

$$
N\left(n, t_{1}, t_{2}, t_{3}, t_{4}\right) \approx \frac{1}{n}\left(2^{n-4}-\left[n / p_{1} \geq 4\right] 2^{n / p_{1}-4}\right),
$$

and for the last four cases of $\left(t_{1}, t_{2}, t_{3}, t_{4}\right)$ we have

$$
N\left(n, t_{1}, t_{2}, t_{3}, t_{4}\right) \approx \frac{1}{n}\left(2^{n-4}-\left[n / p_{1} \geq 4\right] 2^{n / p_{1}-4}-F\left(n / 2, t_{1}^{\prime}, t_{2}^{\prime}\right)+F\left(n / 2 p_{1}, t_{1}^{\prime}, t_{2}^{\prime}\right)\right),
$$

where $t_{1}^{\prime}, t_{2}^{\prime} \in \mathbb{F}_{2}$ vary depending on $p_{1} \equiv 1$ or $3(\bmod 4)$.

Case 3: $n=2^{k_{0}} p_{1}^{k_{1}} p_{2}^{k_{2}}$.

Assume that $n=2^{k_{0}} p_{1}^{k_{1}} p_{2}^{k_{2}}$ where $k_{0}, k_{1}, k_{2} \geq 1$. Then the odd divisors of $n$ are $d=1, p_{1}, p_{2}, p_{1} . p_{2}$. In this case, let $S_{1}=\left\{p_{1}, p_{2}\right\}$ and $S_{2}=\left\{p_{1} . p_{2}\right\}$. Then

$$
\mu(d)= \begin{cases}(-1)^{1}=-1 & \text { if } d \in S_{1} \\ (-1)^{2}=1 & \text { if } d \in S_{2}\end{cases}
$$

Thus $\mu(d)=(-1)^{i}$, for all $d \in S_{i}$, where $i=1,2$. For any $d \in S_{i}$, where $i=1,2$, there exist some $k \in S=\{1,3,5,7\}$ such that $d \equiv k(\bmod 8)$. Also

$$
d \equiv k^{\prime} \quad(\bmod 4) \text { for } k^{\prime} \in S^{\prime}=\{1,3\}
$$

To estimate $N\left(n, t_{1}, t_{2}, t_{3}, t_{4}\right)$ let $\left(t_{1}, t_{2}, t_{3}, t_{4}\right)$ be given in group $(i)$. Without loss 
of generality, let $\left(t_{1}, t_{2}, t_{3}, t_{4}\right)=(1,1,1,0)$. Then

$$
\begin{aligned}
& n N(n, 1,1,1,0)=F(n, 1,1,1,0)+\sum_{\substack { q=1 \\
\begin{subarray}{c}{d \in S_{q} \\
d \equiv 1{ q = 1 \\
\begin{subarray} { c } { d \in S _ { q } \\
d \equiv 1 } }\end{subarray}} \mu(d) F(n / d, 1,1,1,0) \\
& +\sum_{q=1}^{2} \sum_{\substack{d \in S_{q} \\
d=3}} \mu(d) F(n / d, 1,0,0,0)+\sum_{q=1}^{2} \sum_{\substack{d \in S_{q} \\
d=5}} \mu(d) F(n / d, 1,1,1,1) \\
& +\sum_{q=1}^{2} \sum_{\substack{d \in S_{q} \\
d \equiv 7}} \mu(d) F(n / d, 1,0,0,1)
\end{aligned}
$$

Since $F\left(n / d, t_{1}, t_{2}, t_{3}, t_{4}\right)=[n / d \geq 4] 2^{n / d-4}$, and $\mu(d)=(-1)^{q}$, for all $d \in S_{q}$, the last equation simplifies to

$$
\begin{aligned}
& n N(n, 1,1,1,0) \approx 2^{n-4}+\sum_{\substack { q=1 \\
\begin{subarray}{c}{d \in S_{q} \\
d \equiv 1{ q = 1 \\
\begin{subarray} { c } { d \in S _ { q } \\
d \equiv 1 } }\end{subarray}}(-1)^{q}\left[\frac{n}{d} \geq 4\right] 2^{n / d-4} \\
& +\sum_{q=1}^{2} \sum_{\substack{d \in S_{q} \\
d=3}}(-1)^{q}\left[\frac{n}{d} \geq 4\right] 2^{n / d-4}+\sum_{q=1}^{2} \sum_{\substack{d \in S_{q} \\
d=5}}(-1)^{q}\left[\frac{n}{d} \geq 4\right] 2^{n / d-4} \\
& +\sum_{q=1}^{2} \sum_{\substack{d \in S_{q} \\
d \equiv 7}}(-1)^{q}\left[\frac{n}{d} \geq 4\right] 2^{n / d-4}
\end{aligned}
$$

Therefore

$$
\begin{aligned}
n N(n, 1,1,1,0) & \approx 2^{n-4}+\sum_{q=1}^{2} \sum_{d \in S_{q}}(-1)^{q}\left[\frac{n}{d} \geq 4\right]\left([d \equiv 1] 2^{n / d-4}\right. \\
& \left.+[d \equiv 3] 2^{n / d-4}+[d \equiv 5] 2^{n / d-4}+[d \equiv 7] 2^{n / d-4}\right) \\
& =2^{n-4}+\sum_{q=1}^{2} \sum_{d \in S_{q}} \sum_{k \in S}(-1)^{q}\left[\frac{n}{d} \geq 4\right][d \equiv k] 2^{n / d-4} .
\end{aligned}
$$

Since for any $d \in S_{q}$, where $q=1,2$, there exist a unique $k \in S=\{1,3,5,7\}$ such 
that $d \equiv k(\bmod 8)$, we conclude that

$$
N(n, 1,1,1,0) \approx \frac{1}{n}\left(2^{n-4}+\sum_{q=1}^{2} \sum_{d \in S_{q}}\left[\frac{n}{d} \geq 4\right](-1)^{q} 2^{\frac{n}{d}-4}\right) .
$$

For any other $\left(t_{1}, t_{2}, t_{3}, t_{4}\right)$ in group $(i)$ we have the same estimate for the number $N\left(n, t_{1}, t_{2}, t_{3}, t_{4}\right)$. Thus

$$
N\left(n, t_{1}, t_{2}, t_{3}, t_{4}\right) \approx \frac{1}{n}\left(2^{n-4}+\sum_{q=1}^{2} \sum_{d \in S_{q}}\left[\frac{n}{d} \geq 4\right](-1)^{q} 2^{\frac{n}{d}-4}\right)
$$

for all $\left(t_{1}, t_{2}, t_{3}, t_{4}\right)$ in group $(i)$.

Let $\left(t_{1}, t_{2}, t_{3}, t_{4}\right)$ be in group $(i i)$, or $\left(t_{1}, t_{2}, t_{3}, t_{4}\right)=(0,0,1,0)$. By Theorem $50(i i)$,

$$
\begin{aligned}
n N(n, 0,0,1,0) & =\sum_{\substack{d \mid n \\
d \text { odd }}} \mu(d) F(n / d, 0,0,1,0) \\
& =F(n, 0,0,1,0)+\sum_{q=1}^{2} \sum_{d \in S_{q}} \mu(d) F(n / d, 0,0,1,0),
\end{aligned}
$$

which gives us

$$
N(n, 0,0,1,0) \approx \frac{1}{n}\left(2^{n-4}+\sum_{q=1}^{2} \sum_{d \in S_{q}}\left[\frac{n}{d} \geq 4\right](-1)^{q} 2^{\frac{n}{d}-4}\right) .
$$

In a similar way, for $\left(t_{1}, t_{2}, t_{3}, t_{4}\right)$ in group $(i i i)$ we have

$$
N(n, 0,0,1,1) \approx \frac{1}{n}\left(2^{n-4}+\sum_{q=1}^{2} \sum_{d \in S_{q}}\left[\frac{n}{d} \geq 4\right](-1)^{q} 2^{\frac{n}{d}-4}\right) .
$$

For all $\left(t_{1}, t_{2}, t_{3}, t_{4}\right)$ in group $(i v)$, an argument similar to the group $(i)$ gives us the same estimate for the number $N\left(n, t_{1}, t_{2}, t_{3}, t_{4}\right)$. Let $a \equiv b(\bmod 4)$ be shortened as 
$a \equiv b$. Clearly for any $d \in S_{q}$, where $q=1,2$, we have $d \equiv 1$, or $d \equiv 3$. In other words, for any $d \in S_{q}$ there exist one $k^{\prime} \in S^{\prime}=\{1,3\}$ such that $d \equiv k^{\prime}$. Suppose that $\left(t_{1}, t_{2}, t_{3}, t_{4}\right)$ be in group $(v)$, and let $\left(t_{1}, t_{2}, t_{3}, t_{4}\right)=(0,1,1,1)$. Then by Theorem $50(v)$ we have

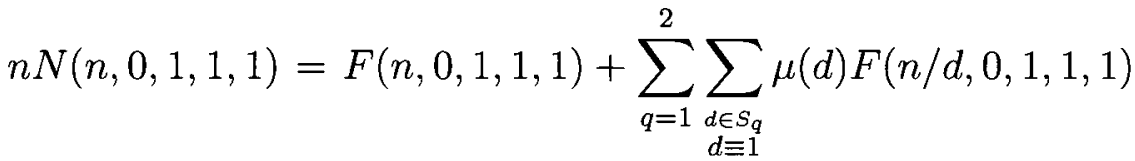

$$
\begin{aligned}
& +\sum_{q=1}^{2} \sum_{\substack{d \in S_{q} \\
d \equiv 3}} \mu(d) F(n / d, 0,1,1,0) \\
& =2^{n-4}+\sum_{q=1}^{2} \sum_{d \in S_{q}}(-1)^{q}\left[\frac{n}{d} \geq 4\right][d \equiv 1] 2^{n / d-4} \\
& +\sum_{q=1}^{2} \sum_{d \in S_{q}}(-1)^{q}\left[\frac{n}{d} \geq 4\right][d \equiv 3] 2^{n / d-4} \\
& =2^{n-4}+\sum_{q=1}^{2} \sum_{d \in S_{q}} \sum_{k^{\prime} \in S^{\prime}}(-1)^{q}\left[\frac{n}{d} \geq 4\right]\left[d \equiv k^{\prime}\right] 2^{n / d-4} \\
& \approx 2^{n-4}+\sum_{q=1}^{2} \sum_{d \in S_{q}}(-1)^{q}\left[\frac{n}{d} \geq 4\right] 2^{n / d-4} \text {. }
\end{aligned}
$$

This implies that

$$
N(n, 0,1,1,1) \approx \frac{1}{n}\left(2^{n-4}+\sum_{q=1}^{2} \sum_{d \in S_{q}}\left[\frac{n}{d} \geq 4\right](-1)^{q} 2^{\frac{n}{d}-4}\right) .
$$

Also for the other case in group $(v)$ we have

$$
N(n, 0,1,1,0) \approx \frac{1}{n}\left(2^{n-4}+\sum_{q=1}^{2} \sum_{d \in S_{q}}\left[\frac{n}{d} \geq 4\right](-1)^{q} 2^{\frac{n}{d}-4}\right) .
$$

Therefore we have the same estimate for the first 12 cases in groups $(i)-(v)$. 
Let us check the rest of the groups. In group $(v i)$ we have $\left(t_{1}, t_{2}, t_{3}, t_{4}\right)=(0,0,0,0)$. It is clear that any $d \in S_{q}$ where $q=1,2$, is odd and $n / d$ is even. Then by Theorem $50(v i)$ we have

$$
\begin{aligned}
n N(n, 0,0,0,0) & =F(n, 0,0,0,0)+\sum_{q=1}^{2} \sum_{d \in S_{q}} \mu(d) F(n / d, 0,0,0,0) \\
& -F(n / 2,0,0)-\sum_{q=1}^{2} \sum_{d \in S_{q}} \mu(d) F(n / 2 d, 0,0) .
\end{aligned}
$$

If we use the estimates for $F(n, 0,0,0,0)$ and $F(n / d, 0,0,0,0)$, and let $\mu(d)=(-1)^{q}$, where $d \in S_{q}$, then

$$
\begin{aligned}
n N(n, 0,0,0,0) \approx & 2^{n-4}+\sum_{q=1}^{2} \sum_{d \in S_{q}}(-1)^{q}\left[\frac{n}{d} \geq 4\right] 2^{n / d-4} \\
& -F(n / 2,0,0)-\sum_{q=1}^{2} \sum_{d \in S_{q}}(-1)^{q} F(n / 2 d, 0,0) \\
& \approx 2^{n-4}-F(n / 2,0,0) \\
& +\sum_{q=1}^{2} \sum_{d \in S_{q}}(-1)^{q}\left(\left[\frac{n}{d} \geq 4\right] 2^{n / d-4}-F(n / 2 d, 0,0)\right) .
\end{aligned}
$$

Briefly in group (vi) we have

$$
\begin{aligned}
N(n, 0,0,0,0) & \approx \frac{1}{n}\left(2^{n-4}+\sum_{q=1}^{2} \sum_{d \in S_{q}}\left[\frac{n}{d} \geq 4\right](-1)^{q} 2^{n / d-4}\right) \\
& -\frac{1}{n}\left(F(n / 2,0,0)+\sum_{q=1}^{s} \sum_{d \in S_{q}}(-1)^{q} F(n / 2 d, 0,0)\right) .
\end{aligned}
$$


The following is a similar result for $\left(t_{1}, t_{2}, t_{3}, t_{4}\right)$ in group (vii)

$$
\begin{aligned}
N(n, 0,0,0,1) & \approx \frac{1}{n}\left(2^{n-4}+\sum_{q=1}^{2} \sum_{d \in S_{q}}\left[\frac{n}{d} \geq 4\right](-1)^{q} 2^{n / d-4}\right) \\
& -\frac{1}{n}\left(F(n / d, 0,1)+\sum_{q=1}^{s} \sum_{d \in S_{q}}(-1)^{q} F(n / 2 d, 0,1)\right) .
\end{aligned}
$$

Finally we let $\left(t_{1}, t_{2}, t_{3}, t_{4}\right)=(0,1,0,0)$, which is in group (viii). Then

$$
\begin{aligned}
n N(n, 0,1,0,0) & =F(n, 0,1,0,0)+\sum_{q=1}^{2} \sum_{\substack{d \in S_{q} \\
d \equiv 1}} \mu(d) F(n / d, 0,1,0,0) \\
& -F(n / 2,1,0)-\sum_{\substack { q=1 \\
\begin{subarray}{c}{d \in S_{q} \\
d=1{ q = 1 \\
\begin{subarray} { c } { d \in S _ { q } \\
d = 1 } }\end{subarray}}^{2} \mu(d) F(n / 2 d, 1,0) \\
& +\sum_{q=1}^{2} \sum_{\substack{d \in S_{q} \\
d \equiv 3}} \mu(d) F(n / d, 0,1,0,1)-\sum_{q=1}^{2} \sum_{\substack{d \in S_{q} \\
d \equiv 3}} \mu(d) F(n / 2 d, 1,1) .
\end{aligned}
$$

Therefore

$$
\begin{aligned}
& n N(n, 0,1,0,0) \approx 2^{n-4}+\sum_{q=1}^{2} \sum_{d \in S_{q}}[d \equiv 1](-1)^{q}\left[\frac{n}{d} \geq 4\right] 2^{n / d-4} \\
&-F(n / 2,1,0)-\sum_{q=1}^{2} \sum_{d \in S_{q}}[d \equiv 1](-1)^{q} F(n / 2 d, 1,0) \\
&+\sum_{q=1}^{2} \sum_{d \in S_{q}}[d \equiv 3](-1)^{q}\left[\frac{n}{d} \geq 4\right] 2^{n / d-4} \\
&-\sum_{q=1}^{2} \sum_{d \in S_{q}}[d \equiv 3](-1)^{q} F(n / 2 d, 1,1) .
\end{aligned}
$$


This can be given as

$$
\begin{aligned}
n N(n, 0,1,0,0) \approx & 2^{n-4}-F(n / 2,1,0) \\
& +\sum_{q=1}^{2} \sum_{d \in S_{q}} \sum_{k^{\prime} \in S^{\prime}}\left[d \equiv k^{\prime}\right](-1)^{q}\left[\frac{n}{d} \geq 4\right] 2^{n / d-4} \\
& -\sum_{q=1}^{2} \sum_{d \in S_{q}}(-1)^{q}([d \equiv 1] F(n / 2 d, 1,0)+[d \equiv 3] F(n / 2 d, 1,1)),
\end{aligned}
$$

which implies that

$$
\begin{aligned}
N(n, 0,1,0,0) & \approx \frac{1}{n}\left(2^{n-4}+\sum_{q=1}^{2} \sum_{d \in S_{q}}(-1)^{q}\left[\frac{n}{d} \geq 4\right] 2^{n / d-4}-F(n / 2,1,0)\right) \\
& -\frac{1}{n}\left(\sum_{q=1}^{2} \sum_{d \in S_{q}}(-1)^{q}([d \equiv 1] F(n / 2 d, 1,0)+[d \equiv 3] F(n / 2 d, 1,1))\right) .
\end{aligned}
$$

For the other case in group (viii) we have

$$
\begin{aligned}
N(n, 0,1,0,1) & \approx \frac{1}{n}\left(2^{n-4}+\sum_{q=1}^{2} \sum_{d \in S_{q}}(-1)^{q}\left[\frac{n}{d} \geq 4\right] 2^{n / d-4}-F(n / 2,1,1)\right) \\
& -\frac{1}{n}\left(\sum_{q=1}^{2} \sum_{d \in S_{q}}(-1)^{q}([d \equiv 1] F(n / 2 d, 1,1)+[d \equiv 3] F(n / 2 d, 1,0))\right) .
\end{aligned}
$$

Case 4: $n=2^{k_{0}} p_{1}{ }^{k_{1}} \ldots p_{s}^{k_{s}}$.

In general we let $n=2^{k_{0}} p_{1}{ }^{k_{1}} \ldots p_{s}{ }^{k_{s}}$, where $k_{0}, \ldots, k_{s} \geq 1$, and $p_{1}, \ldots, p_{s}$ are odd prime divisors of $n$. We find an approximation for $N\left(n, t_{1}, t_{2}, t_{3}, t_{4}\right)$. Using the prime factors of $n$ we define $S_{1}=\left\{p_{1}, \ldots, p_{s}\right\}$. For $2 \leq q \leq s$ let $S_{q}$ be the set of all $d \mid n$, where $d$ is the product of exactly $q$ distinct odd prime $p_{i} \in S_{1}$, for $i \in\{1, \ldots, s\}$.

Clearly, $\mu(d)=-1$, for all $d \in S_{1}$. For a given $q \in\{2, \ldots, s\}$ if $d \in S_{q}$ then by 
the definition of Möbius function we have $\mu(d)=(-1)^{q}$. An argument similar to the case $n=2^{k_{0}} p_{1}{ }^{k_{1}} p_{2}{ }^{k_{2}}$ proves that for all 12 cases of $\left(t_{1}, t_{2}, t_{3}, t_{4}\right)$ which are in groups $(i)-(v)$ we have the same estimate for the number $N\left(n, t_{1}, t_{2}, t_{3}, t_{4}\right)$, which can be given as

$$
N\left(n, t_{1}, t_{2}, t_{3}, t_{4}\right) \approx \frac{1}{n}\left(2^{n-4}+\sum_{q=1}^{s} \sum_{d \in S_{q}}\left[\frac{n}{d} \geq 4\right](-1)^{q} 2^{n / d-4}\right) .
$$

In groups (vi) and (vii) we have

$$
\begin{aligned}
N(n, 0,0,0,0) \approx & \frac{1}{n}\left(2^{n-4}+\sum_{q=1}^{s} \sum_{d \in S_{q}}\left[\frac{n}{d} \geq 4\right](-1)^{q} 2^{n / d-4}\right) \\
& -\frac{1}{n}\left(F(n / 2,0,0)+\sum_{q=1}^{s} \sum_{d \in S_{q}}(-1)^{q} F(n / 2 d, 0,0)\right), \\
N(n, 0,0,0,1) \approx & \frac{1}{n}\left(2^{n-4}+\sum_{q=1}^{s} \sum_{d \in S_{q}}\left[\frac{n}{d} \geq 4\right](-1)^{q} 2^{n / d-4}\right) \\
& -\frac{1}{n}\left(F(n / 2,0,1)+\sum_{q=1}^{s} \sum_{d \in S_{q}}(-1)^{q} F(n / 2 d, 0,1)\right) .
\end{aligned}
$$

For $\left(t_{1}, t_{2}, t_{3}, t_{4}\right)$ from the groups $(v i)$ and (vii) we have $t_{1}=t_{2}=t_{3}=0$, and $t_{4}=0$ or 1 , respectively. Therefore $\left(0,0,0, t_{4}\right)$ is in group $(v i)$ or $(v i i)$, where $t_{4} \in \mathbb{F}_{2}$, and if we combine the last two equations, then for groups (vi) and (vii) we have

$$
\begin{aligned}
N\left(n, 0,0,0, t_{4}\right) & \approx \frac{1}{n}\left(2^{n-4}+\sum_{q=1}^{s} \sum_{d \in S_{q}}\left[\frac{n}{d} \geq 4\right](-1)^{q} 2^{n / d-4}\right) \\
& -\frac{1}{n}\left(F\left(n / d, 0, t_{4}\right)+\sum_{q=1}^{s} \sum_{d \in S_{q}}(-1)^{q} F\left(n / 2 d, 0, t_{4}\right)\right) .
\end{aligned}
$$

Let $a \equiv b(\bmod 4)$ be shortened as $a \equiv b$. Finally we take $\left(t_{1}, t_{2}, t_{3}, t_{4}\right)$ from group 
(viii). If we let $\left(t_{1}, t_{2}, t_{3}, t_{4}\right)=(0,1,0,0)$, then the estimate of $N\left(n, t_{1}, t_{2}, t_{3}, t_{4}\right)$ can be given as

$$
\begin{aligned}
N(n, 0,1,0,0) \approx & \frac{1}{n}\left(2^{n-4}+\sum_{q=1}^{s} \sum_{d \in S_{q}}(-1)^{q}\left[\frac{n}{d} \geq 4\right] 2^{n / d-4}-F(n / 2,1,0)\right) \\
& -\frac{1}{n}\left(\sum_{q=1}^{s} \sum_{d \in S_{q}}(-1)^{q}([d \equiv 1] F(n / 2 d, 1,0)+[d \equiv 3] F(n / 2 d, 1,1))\right),
\end{aligned}
$$

For the other case in group (viii) we have

$$
\begin{aligned}
N(n, 0,1,0,1) & \approx \frac{1}{n}\left(2^{n-4}+\sum_{q=1}^{s} \sum_{d \in S_{q}}(-1)^{q}\left[\frac{n}{d} \geq 4\right] 2^{n / d-4}-F(n / 2,1,1)\right) \\
& -\frac{1}{n}\left(\sum_{q=1}^{s} \sum_{d \in S_{q}}(-1)^{q}([d \equiv 1] F(n / 2 d, 1,1)+[d \equiv 3] F(n / 2 d, 1,0))\right) .
\end{aligned}
$$

Again we can consider $\left(0,1,0, t_{4}\right)$ in the group (viii), where $t_{4} \in \mathbb{F}_{2}$. Then one can combine the last two equations, and obtain the following equation

$$
\begin{aligned}
N\left(n, 0,1,0, t_{4}\right) & \approx \frac{1}{n}\left(2^{n-4}+\sum_{q=1}^{s} \sum_{d \in S_{q}}(-1)^{q}\left[\frac{n}{d} \geq 4\right] 2^{n / d-4}-F\left(n / 2,1, t_{4}\right)\right) \\
& -\frac{1}{n}\left(\sum_{q=1}^{s} \sum_{d \in S_{q}}(-1)^{q}\left([d \equiv 1] F\left(n / 2 d, 1, t_{4}\right)+[d \equiv 3] F\left(n / 2 d, 1, \bar{t}_{4}\right)\right)\right),
\end{aligned}
$$

where $\overline{t_{4}}=1+t_{4}(\bmod 2)$.

\subsection{Experimental Results}

We let $n=14,16, \ldots, 24$ and then we computed our estimate for $N\left(n, t_{1}, t_{2}, t_{3}, t_{4}\right)$ for all 16 possible cases of $\left(t_{1}, t_{2}, t_{3}, t_{4}\right)$. For the given values of $n$, by a Maple code, we 
computed the exact values of $N\left(n, t_{1}, t_{2}, t_{3}, t_{4}\right)$ in order to compare with our results. The results are given in the following tables. For the case $n=2^{k_{0}}$ where $k_{0} \geq 3$, we have the best approximation. This is true because in this case the only odd divisor of $n$ is $d=1$. Indeed, for $n=2^{k_{0}}$ and $\left(t_{1}, t_{2}, t_{3}, t_{4}\right)$ from groups $(i)-(v)$ of Theorem 50 we have

$$
n N\left(n, t_{1}, t_{2}, t_{3}, t_{4}\right)=F\left(n, t_{1}, t_{2}, t_{3}, t_{4}\right) \approx 2^{n-4}
$$

or $N\left(n, t_{1}, t_{2}, t_{3}, t_{4}\right) \approx 2^{n-4-k_{0}}$, which is the exact value of $N\left(n, t_{1}, t_{2}, t_{3}, t_{4}\right)$ in most of the first 12 cases. For the other 4 cases we have

$$
n N\left(n, t_{1}, t_{2}, t_{3}, t_{4}\right)=F\left(n, t_{1}, t_{2}, t_{3}, t_{4}\right)-F\left(n, t_{1}^{\prime}, t_{2}^{\prime}\right) \approx 2^{n-4}-F\left(n, t_{1}^{\prime}, t_{2}^{\prime}\right),
$$

which has a small error. For the other cases of $n$ we do not have the exact value, but very good approximation of $N\left(n, t_{1}, t_{2}, t_{3}, t_{4}\right)$.

To compare our estimate and the exact value of $N\left(n, t_{1}, t_{2}, t_{3}, t_{4}\right)$ in each table we let

$$
\operatorname{error}=\operatorname{exact}\left(N\left(n, t_{1}, t_{2}, t_{3}, t_{4}\right)\right)-\operatorname{estimate}\left(N\left(n, t_{1}, t_{2}, t_{3}, t_{4}\right)\right)
$$

Then for different case number $i=1, \ldots, 16$ we define

$$
\rho_{i}= \begin{cases}\frac{\text { estimate of } N}{\text { exact } N} & \text { if error is positive } \\ \frac{\text { exact } N}{\text { estimate of } N} & \text { if error is negative } \\ 1 & \text { if error is zero. }\end{cases}
$$

Now assume that $\rho=\min \left\{\rho_{1}, \ldots, \rho_{16}\right\}$. Therefore $\rho \leq 1$, and if $\rho=1$ then our estimate is the exact value of $N\left(n, t_{1}, t_{2}, t_{3}, t_{4}\right)$. For different degree $n$ the values of $\rho$ are given in Table 7.7. Numerical results gives the evidence that as $n$ grows $\rho$ gets closer to one, which means for large $n$ our estimation of $N\left(n, t_{1}, t_{2}, t_{3}, t_{4}\right)$ is closer to 
its exact value. One can see this from Theorem 50 . In the first 12 cases of Theorem 50 we have

$$
\begin{aligned}
n N\left(n, t_{1}, t_{2}, t_{3}, t_{4}\right) & =F\left(n, t_{1}, t_{2}, t_{3}, t_{4}\right)+\sum_{\begin{array}{c}
d \mid n \\
d \text { odd }
\end{array}} \mu(d) F\left(n / d, t_{1}^{\prime}, t_{2}^{\prime}, t_{3}^{\prime}, t_{4}^{\prime}\right) \\
& \approx 2^{n-4}+\sum_{\substack{d \mid n \\
d \text { odd }}} \mu(d)[n / d \geq 4] 2^{n / d-4} .
\end{aligned}
$$

In the second term we divide $n$ by $d$, and for a large $n$ the term $2^{n-4}$ has a larger weight comparing with different $2^{n / d-4}$ in the second term. This is also true for the remaining four cases.

Table 7.1: Different values of $N\left(14, t_{1}, t_{2}, t_{3}, t_{4}\right)$, where $t_{i}=0,1$.

\begin{tabular}{|c|c|c|c|c|c|}
\hline Case No. & $\left(t_{1}, t_{2}, t_{3}, t_{4}\right)$ & our estimate & exact value & error & $\rho_{i}$ \\
\hline 1 & $(1,1,1,0)$ & 73.14 & 72 & -1.14 & 0.9844 \\
\hline 2 & $(1,0,0,1)$ & 73.14 & 72 & -1.14 & 0.9844 \\
\hline 3 & $(1,1,1,1)$ & 73.14 & 72 & -1.14 & 0.9844 \\
\hline 4 & $(1,0,0,0)$ & 73.14 & 72 & -1.14 & 0.9844 \\
\hline 5 & $(0,0,1,0)$ & 73.14 & 74 & 0.86 & 0.9883 \\
\hline 6 & $(0,0,1,1)$ & 73.14 & 70 & -3.14 & 0.9571 \\
\hline 7 & $(1,1,0,0)$ & 73.14 & 72 & -1.14 & 0.9844 \\
\hline 8 & $(1,0,1,1)$ & 73.14 & 69 & -4.14 & 0.9433 \\
\hline 9 & $(1,1,0,1)$ & 73.14 & 72 & -1.14 & 0.9844 \\
\hline 10 & $(1,0,1,0)$ & 73.14 & 84 & 10.86 & 0.8707 \\
\hline 11 & $(0,1,1,1)$ & 73.14 & 70 & -3.14 & 0.9571 \\
\hline 12 & $(0,1,1,0)$ & 73.14 & 74 & 0.86 & 0.9883 \\
\hline 13 & $(0,0,0,0)$ & 71.71 & 74 & 2.29 & 0.9691 \\
\hline 14 & $(0,0,0,1)$ & 72.28 & 70 & -2.28 & 0.9685 \\
\hline 15 & $(0,1,0,0)$ & 72.28 & 70 & -2.28 & 0.9685 \\
\hline 16 & $(0,1,0,1)$ & 71.71 & 74 & 2.29 & 0.9691 \\
\hline Total & & 1165.66 & 1161 & & \\
\hline
\end{tabular}


Table 7.2: Different values of $N\left(16, t_{1}, t_{2}, t_{3}, t_{4}\right)$, where $t_{i}=0,1$.

\begin{tabular}{|c|c|c|c|c|c|}
\hline Case No. & $\left(t_{1}, t_{2}, t_{3}, t_{4}\right)$ & our estimate & exact value & error & $\rho_{i}$ \\
\hline 1 & $(1,1,1,0)$ & 256 & 260 & 4 & 0.9846 \\
\hline 2 & $(1,0,0,1)$ & 256 & 260 & 4 & 0.9846 \\
\hline 3 & $(1,1,1,1)$ & 256 & 252 & -4 & 0.9843 \\
\hline 4 & $(1,0,0,0)$ & 256 & 252 & -4 & 0.9843 \\
\hline 5 & $(0,0,1,0)$ & 256 & 256 & 0 & 1 \\
\hline 6 & $(0,0,1,1)$ & 256 & 256 & 0 & 1 \\
\hline 7 & $(1,1,0,0)$ & 256 & 256 & 0 & 1 \\
\hline 8 & $(1,0,1,1)$ & 256 & 256 & 0 & 1 \\
\hline 9 & $(1,1,0,1)$ & 256 & 256 & 0 & 1 \\
\hline 10 & $(1,0,1,0)$ & 256 & 256 & 0 & 1 \\
\hline 11 & $(0,1,1,1)$ & 256 & 264 & 8 & 0.9697 \\
\hline 12 & $(0,1,1,0)$ & 256 & 264 & 8 & 0.9697 \\
\hline 13 & $(0,0,0,0)$ & 252.5 & 240 & -12.5 & 0.9504 \\
\hline 14 & $(0,0,0,1)$ & 251.5 & 256 & -4.5 & 0.9824 \\
\hline 15 & $(0,1,0,0)$ & 252 & 248 & -4 & 0.9841 \\
\hline 16 & $(0,1,0,1)$ & 252 & 248 & -4 & 0.9841 \\
\hline Total & & 4080 & 4080 & & \\
\hline
\end{tabular}

Table 7.3: Different values of $N\left(18, t_{1}, t_{2}, t_{3}, t_{4}\right)$, where $t_{i}=0,1$.

\begin{tabular}{|c|c|c|c|c|c|}
\hline Case No. & $\left(t_{1}, t_{2}, t_{3}, t_{4}\right)$ & our estimate & exact value & error & $\rho_{i}$ \\
\hline 1 & $(1,1,1,0)$ & 910 & 917 & 7 & 0.9923 \\
\hline 2 & $(1,0,0,1)$ & 910 & 896 & -14 & 0.9846 \\
\hline 3 & $(1,1,1,1)$ & 910 & 917 & 7 & 0.9923 \\
\hline 4 & $(1,0,0,0)$ & 910 & 896 & -14 & 0.9846 \\
\hline 5 & $(0,0,1,0)$ & 910 & 921 & 11 & 0.9881 \\
\hline 6 & $(0,0,1,1)$ & 910 & 892 & -18 & 0.9802 \\
\hline 7 & $(1,1,0,0)$ & 910 & 927 & 17 & 0.9816 \\
\hline 8 & $(1,0,1,1)$ & 910 & 917 & 17 & 0.9816 \\
\hline 9 & $(1,1,0,1)$ & 910 & 893 & -17 & 0.9813 \\
\hline 10 & $(1,0,1,0)$ & 910 & 917 & 7 & 0.9923 \\
\hline 11 & $(0,1,1,1)$ & 910 & 921 & 11 & 0.9881 \\
\hline 12 & $(0,1,1,0)$ & 910 & 892 & -18 & 0.9802 \\
\hline 13 & $(0,0,0,0)$ & 906.89 & 913 & 6.11 & 0.9933 \\
\hline 14 & $(0,0,0,1)$ & 903.5 & 900 & -3.5 & 0.9961 \\
\hline 15 & $(0,1,0,0)$ & 906.44 & 913 & 6.56 & 0.9928 \\
\hline 16 & $(0,1,0,1)$ & 906.44 & 900 & -6.44 & 0.9928 \\
\hline Total & & 14543.27 & 14532 & & \\
\hline
\end{tabular}


Table 7.4: Different values of $N\left(20, t_{1}, t_{2}, t_{3}, t_{4}\right)$, where $t_{i}=0,1$.

\begin{tabular}{|c|c|c|c|c|c|}
\hline Case No. & $\left(t_{1}, t_{2}, t_{3}, t_{4}\right)$ & our estimate & exact value & error & $\rho_{i}$ \\
\hline 1 & $(1,1,1,0)$ & 3276.75 & 3275 & -1.75 & 0.9995 \\
\hline 2 & $(1,0,0,1)$ & 3276.75 & 3304 & 27.25 & 0.9917 \\
\hline 3 & $(1,1,1,1)$ & 3276.75 & 3304 & 27.25 & 0.9917 \\
\hline 4 & $(1,0,0,0)$ & 3276.75 & 3275 & -1.75 & 0.9995 \\
\hline 5 & $(0,0,1,0)$ & 3276.75 & 3264 & -12.75 & 0.9961 \\
\hline 6 & $(0,0,1,1)$ & 3276.75 & 3315 & 38.25 & 0.9884 \\
\hline 7 & $(1,1,0,0)$ & 3276.75 & 3264 & -12.75 & 0.9961 \\
\hline 8 & $(1,0,1,1)$ & 3276.75 & 3264 & -12.75 & 0.9961 \\
\hline 9 & $(1,1,0,1)$ & 3276.75 & 3264 & -12.75 & 0.9961 \\
\hline 10 & $(1,0,1,0)$ & 3276.75 & 3264 & -12.75 & 0.9961 \\
\hline 11 & $(0,1,1,1)$ & 3276.75 & 3264 & -12.75 & 0.9961 \\
\hline 12 & $(0,1,1,0)$ & 3276.75 & 3264 & -12.75 & 0.9961 \\
\hline 13 & $(0,0,0,0)$ & 3264 & 3264 & 0 & 1 \\
\hline 14 & $(0,0,0,1)$ & 3264 & 3264 & 0 & 1 \\
\hline 15 & $(0,1,0,0)$ & 3264.75 & 3280 & 15.25 & 0.9953 \\
\hline 16 & $(0,1,0,1)$ & 3263.25 & 3248 & -15.25 & 0.9953 \\
\hline Total & & 53350 & 52377 & & \\
\hline
\end{tabular}

Table 7.5: Different values of $N\left(22, t_{1}, t_{2}, t_{3}, t_{4}\right)$, where $t_{i}=0,1$.

\begin{tabular}{|c|c|c|c|c|c|}
\hline Case No. & $\left(t_{1}, t_{2}, t_{3}, t_{4}\right)$ & our estimate & exact value & error & $\rho_{i}$ \\
\hline 1 & $(1,1,1,0)$ & 11915.64 & 11904 & -11.64 & 0.999 \\
\hline 2 & $(1,0,0,1)$ & 11915.64 & 11904 & -11.64 & 0.999 \\
\hline 3 & $(1,1,1,1)$ & 11915.64 & 11904 & -11.64 & 0.999 \\
\hline 4 & $(1,0,0,0)$ & 11915.64 & 11904 & -11.64 & 0.999 \\
\hline 5 & $(0,0,1,0)$ & 11915.64 & 11992 & 76.36 & 0.9936 \\
\hline 6 & $(0,0,1,1)$ & 11915.64 & 11816 & -99.64 & 0.9916 \\
\hline 7 & $(1,1,0,0)$ & 11915.64 & 11904 & -11.64 & 0.999 \\
\hline 8 & $(1,0,1,1)$ & 11915.64 & 11952 & 36.36 & 0.9969 \\
\hline 9 & $(1,1,0,1)$ & 11915.64 & 11904 & -11.64 & 0.999 \\
\hline 10 & $(1,0,1,0)$ & 11915.64 & 11949 & 33.36 & 0.9972 \\
\hline 11 & $(0,1,1,1)$ & 11915.64 & 11816 & -99.64 & 0.9916 \\
\hline 12 & $(0,1,1,0)$ & 11915.64 & 11992 & 76.36 & 0.9936 \\
\hline 13 & $(0,0,0,0)$ & 11893.14 & 11928 & 34.86 & 0.997 \\
\hline 14 & $(0,0,0,1)$ & 11891.68 & 11880 & -11.68 & 0.999 \\
\hline 15 & $(0,1,0,0)$ & 11891.73 & 11880 & -11.73 & 0.999 \\
\hline 16 & $(0,1,0,1)$ & 11893.09 & 11928 & 34.91 & 0.997 \\
\hline Total & & 190557.32 & 190557 & & \\
\hline
\end{tabular}


Table 7.6: Different values of $N\left(24, t_{1}, t_{2}, t_{3}, t_{4}\right)$, where $t_{i}=0,1$.

\begin{tabular}{|c|c|c|c|c|c|}
\hline Case No. & $\left(t_{1}, t_{2}, t_{3}, t_{4}\right)$ & our estimate & exact value & error & $\rho_{i}$ \\
\hline 1 & $(1,1,1,0)$ & 43690 & 43759 & 69 & 0.9984 \\
\hline 2 & $(1,0,0,1)$ & 43690 & 43759 & 69 & 0.9984 \\
\hline 3 & $(1,1,1,1)$ & 43690 & 43621 & -69 & 0.9984 \\
\hline 4 & $(1,0,0,0)$ & 43690 & 43621 & -69 & 0.9984 \\
\hline 5 & $(0,0,1,0)$ & 43690 & 43754 & 64 & 0.9985 \\
\hline 6 & $(0,0,1,1)$ & 43690 & 43754 & 64 & 0.9985 \\
\hline 7 & $(1,1,0,0)$ & 43690 & 43690 & 0 & 1 \\
\hline 8 & $(1,0,1,1)$ & 43690 & 43690 & 0 & 1 \\
\hline 9 & $(1,1,0,1)$ & 43690 & 43690 & 0 & 1 \\
\hline 10 & $(1,0,1,0)$ & 43690 & 43690 & 0 & 1 \\
\hline 11 & $(0,1,1,1)$ & 43690 & 43711 & 21 & 0.9995 \\
\hline 12 & $(0,1,1,0)$ & 43690 & 43711 & 21 & 0.9995 \\
\hline 13 & $(0,0,0,0)$ & 43646.25 & 43520 & -126.25 & 0.9971 \\
\hline 14 & $(0,0,0,1)$ & 43648.75 & 43562 & -86.75 & 0.9980 \\
\hline 15 & $(0,1,0,0)$ & 43647.5 & 43669 & 21.5 & 0.9995 \\
\hline 16 & $(0,1,0,1)$ & 43647.5 & 43669 & 21.5 & 0.9995 \\
\hline Total & & 698870 & 698870 & & \\
\hline
\end{tabular}

Table 7.7: Different values of $\rho$

\begin{tabular}{|r|c|}
\hline$n$ & $\rho$ \\
\hline 14 & 0.8707 \\
\hline 16 & 0.9504 \\
\hline 18 & 0.9802 \\
\hline 20 & 0.9884 \\
\hline 22 & 0.9916 \\
\hline 24 & 0.9971 \\
\hline
\end{tabular}




\section{Chapter 8}

\section{Conclusions and Future Directions}

\subsection{Summary of Contributions}

In this thesis we study the number of irreducible polynomials over the finite field $\mathbb{F}_{q}$ of a given degree and some prescribed coefficients. This problem has been studied by several mathematicians for different shapes of the polynomials. In Chapter 3 a generalization of Möbius inversion formula is presented, and later we use that generalization in our study of the number of irreducible polynomials with the first four prescribed coefficients. In Chapter 4 we assume that the trace and constant terms, and we examine the number of irreducible polynomials of degree $n$ over the finite field $\mathbb{F}_{q}$ with those given terms. If $\gamma, c \in \mathbb{F}_{q}$ are the trace and constant terms, respectively, then $N_{\gamma}(n, c, q)$ represents this number. We improve the existing bounds for this number, and we give several results associated to it.

In the remaining chapters of this thesis, we count the number of irreducible polynomials of even degree $n$ over $\mathbb{F}_{2}$ where the coefficients of the terms $x^{n-1}, x^{n-2}, x^{n-3}$ and $x^{n-4}$ are given. This is represented by $N\left(n, t_{1}, t_{2}, t_{3}, t_{4}\right)$, where $t_{i} \in \mathbb{F}_{2}$ are the coefficients of $x^{n-i}$, for $i=1,2,3,4$. Several results towards the exact estimation 
of $N\left(n, t_{1}, t_{2}, t_{3}, t_{4}\right)$ are given. Using these results a very good approximation for $N\left(n, t_{1}, t_{2}, t_{3}, t_{4}\right)$ is given.

\subsection{Future Directions}

We have proved some bounds for the number $N_{\gamma}(n, c, q)$. A natural open problem is to improve these bounds further. Ultimately, we would like to find the exact number of polynomials of degree $n$ over $\mathbb{F}_{q}$ with given trace and norm term. However, this problem seems to be quite difficult. For the number $N(n, c, q)$, Yucas [23] gives a closed formula in terms of Möbius function. For a very particular case, we were able to give the exact value of $N(n, c, q)$ in terms of $n$ and $q$ (see Theorem 34). Can this exact study of $N(n, c, q)$ be done for other values of $n$ ? Perhaps the value of $N(n, c, q)$ can be obtained for some special cases of $n$, like $n=2^{k}$, where $k \geq 2$.

For the problem of studying $N\left(n, t_{1}, t_{2}, t_{3}, t_{4}\right)$, our approximation of the number $N\left(n, t_{1}, t_{2}, t_{3}, t_{4}\right)$ is given in Chapter 7 . A natural way to extend this problem is by completing the counting argument given in Chapter 6 to find the traces of elements of a coset from different categories. By doing this, we will be able to find the exact value of $N\left(n, t_{1}, t_{2}, t_{3}, t_{4}\right)$, for $n$ even. Moreover, a possible continuation of this work is studying $N\left(n, t_{1}, t_{2}, t_{3}, t_{4}\right)$, when the degree $n$ is an odd number. These studies are for polynomials over the finite field $\mathbb{F}_{2}$. We can have similar studies for polynomials over the finite field $\mathbb{F}_{q}$.

Another possible extension is to study the number $N\left(n, t_{1}, \ldots, t_{k}\right)$, that is, the number of irreducible polynomials of degree $n$ over $\mathbb{F}_{2}$ with the first $k$ coefficients prescribed, where $4 \leq k \leq n$. Like our Theorem 50 , it is possible to find the formula for the number $N\left(n, t_{1}, \ldots, t_{k}\right)$ in terms of the number $F\left(n, t_{1}, \ldots, t_{k}\right)$ of elements $\beta \in \mathbb{F}_{2^{n}}$ with the first $k$ traces of $\beta$ are given as $t_{i}$, for $1 \leq i \leq k$. In [24] it is 
conjectured that

$$
F\left(n, t_{1}, \ldots, t_{k}\right)=2^{n-k}+G\left(n, t_{1}, \ldots, t_{k}\right)
$$

where $G\left(n, t_{1}, \ldots, t_{k}\right)$ is in terms of different powers of 2 . Then we could use a similar approach to give a good approximation for the number $N\left(n, t_{1}, \ldots, t_{k}\right)$. 


\section{Bibliography}

[1] E. R. Berlekamp, Bit-serial Reed-Solomon encoders. IEEE Trans. Info. Th., 28 (1982), 869-874.

[2] L. Carlitz, A theorem of Dickson on irreducible polynomials, Proceedings of the American Mathematical Society, 3 (1952), 693-700.

[3] K. Cattell, C. R. Miers, F. Ruskey, M. Serra and J. Sawada, The number of irreducible polynomials over $\mathrm{GF}(2)$ with given trace and subtrace, Journal of Combinatorial mathematics and Combinatorial Computing, 47 (2003), 31-64.

[4] S. D. Cohen, Explicit theorems on generator polynomials, Finite Fields and Their Applications, 11 (2005), 337-357.

[5] S. D. Cohen and M. Presern, Primitive polynomials with prescribed second coefficient, Glasgow Mathematical Journal Trust, 48 (2006), 281-307.

[6] S. D. Cohen, Primitive elements and polynomials with arbitrary trace, Journal of American Mathematics Society, 83 (1990), 1-7.

[7] R. W. Fitzgerald and J. L. Yucas, Irreducible polynomials over GF(2) with three prescribed coefficients, Finite Fields and Their Applications, 9 (2003), 286-299. 
[8] W. Huffman and V. Pless, "Fundamentals of Error-Correcting Codes", Cambridge Univ. Press, Cambridge, first edition, 2003.

[9] C. N. Hsu, The distribution of irreducible polynomials in $F_{q}[t]$, Journal of Number Theory, 61 (1996), 85-96.

[10] IEEE Standard Specifications for Public-Key Cryptography. Technical Report IEEE Std, 1361-2000. IEEE Inc., 3 Park Ave., NY, 10016-5997, USA.

[11] J. A. Jones and J. M. Jones, "Elementary Number Theory", Springer, New York, 1998.

[12] E. N. Kuz'min, On a class of irreducible polynomials over a finite field, Dokl. Akad. Nauk SSSr 313 (3) (1990), 552-555. (Russian: English translation in Soviet Math. Dokl. 42(1) (1991), 45-48.

[13] K. Kononen, M. Moisio, M. Rinta-aho and K. Väänänen, JP Journal of Algebra, Number Theory and Applications, 11 (2008), 223-248.

[14] R. Lidl and H. Niederreiter, "Finite Fields", Cambridge Univ. Press, Cambridge, second edition, 1994.

[15] M. Moisio, Kloosterman sums, elliptic curves, and irreducible polynomials with prescribed trace and norm. Acta Artith., 132 (2008), 329-350.

[16] M. Moisio and K. Ranto, Elliptic curves and explicit enumeration of irreducible polynomials with two coefficients prescribed, Finite Fields and Their Applications, 14 (2008), 798-815.

[17] B. Omidi Koma, D. Panario and Q. Wang, The number of irreducible polynomials of degree n over $\mathbb{F}_{q}$ with given trace and constant terms, Discrete Mathematics, 310 (2010), 1282-1292. 
[18] B. Omidi Koma and D. Panario, Generalizations of Möbius inversion formula; submitted.

[19] F. Ruskey, C. R. Miers and J. Sawada, The number of irreducible polynomials and Lyndon words with given traces, Siam Journal of Discrete Mathematics, 14 (2001), 240-245.

[20] B. Sunar and C. K. Koc, An efficient optimal normal basis Type II multiplier, IEEE Transactions on Computers, 50, No. 1, (2001), 83-87.

[21] R. Swan, Factorization of Polynomials over Finite Fields, Pacific Journal of Mathematics, 12 (1962), 1099-1106.

[22] D. Wan, Generators and irreducible polynomials over finite fields, Mathematics of Computation, 66 (1997), 1195-1212.

[23] J. L. Yucas, Irreducible polynomials over finite fields with prescribed trace/prescribed constant term, Finite Fields and Their Applications, 12 (2006), 211-221.

[24] J. L. Yucas and G. L. Mullen, Irreducible polynomials over GF(2) with prescribed coefficients, Discrete Mathematics, 274 (2004), 265-279. 